\title{
Conventional and High-Strength Headed Bars-Part 2: Data Analysis
}

\author{
by Krishna P. Ghimire, Yun Shao, David Darwin, and Matthew O'Reilly
}

\begin{abstract}
Equations to characterize the anchorage strength of headed bars were developed, incorporating key factors affecting anchorage strength: concrete compressive strength; embedment length; bar diameter; spacing between the bars; and confining reinforcement parallel to the headed bars. Results from tests of 138 exterior beam-column joints, 64 without and 74 with confining reinforcement within the joint region, were used to develop the equations. Concrete compressive strengths ranged from 4050 to 16,030 psi (27.9 to $110.6 \mathrm{MPa}$ ) and bar stresses at failure ranged from 33,100 to $153,160 \mathrm{psi}$ (228 to $1056 \mathrm{MPa}$ ). The bearing area of the headed bars ranged from 3.8 to 9.5 times the area of the bar. Some headed bars contained obstructions adjacent to the head that exceeded the dimensions permitted for HA heads in ACI 318-14 and ASTM A970-13 a but are now permitted by ASTM A970-18. The test results show that headed bar anchorage strength is proportional to the concrete compressive strength raised to the power 0.24. The contribution of confining reinforcement is proportional to the area of confining reinforcement parallel to the headed bar within eight to 10 bar diameters of the headed bar. Headed bars with obstructions larger than those permitted in ACI 318-14 that meet the provisions in ASTM A970-18 exhibit anchorage strengths that are similar to those that meet the provisions in ACI 318-14.
\end{abstract}

Keywords: anchorage; beam-column joints; bond and development; headed bars; high-strength concrete, high-strength steel; reinforcement.

\section{INTRODUCTION}

Headed bars are often used to anchor reinforcing steel as a means of reducing congestion where member geometry precludes adequate anchorage with a straight bar. Prior to the current study (Shao et al. 2016; Ghimire et al. 2018, 2019), only limited results were available on the behavior of headed bars, with no data on high-strength headed bars or applications dealing with high-strength concrete. The design provisions in ACI 318, which first appeared in ACI 318-08, are based on the analysis of a small number of test specimens with a limited range of concrete compressive strengths and bar stresses at failure (DeVries et al. 1998, 1999; Thompson et al. 2005, 2006a,b). None of the tests involved beamcolumn joints. Due to the lack of experimental information, the design provisions for development length of headed reinforcing bars in ACI 318-14 limit the yield strength of headed reinforcing steel to $60,000 \mathrm{psi}(420 \mathrm{MPa})$ and the concrete compressive strength for calculating development length to $6000 \mathrm{psi}$ (40 MPa). ACI 318-14 also requires the clear spacing between the bars to be at least four times the bar diameter and does not account for the contribution of confining reinforcement to anchorage strength. These limits restrict the use of headed bars and prevent the full benefits of higher-strength reinforcing steel and concrete from being realized. The purpose of this study is to identify the primary factors that affect the anchorage strength of the headed bars and develop an expression that characterizes the anchorage strength of the headed bars that incorporates the key parameters and is applicable to the full range of concrete and steel strengths used in construction. The study includes an evaluation of headed bars with obstructions that exceed the dimensions permitted by ACI 318-14.

The equation for the development length of headed bars in tension, $\ell_{d t}$ (in. or mm) in Section 25.4.4.2 of ACI 318-14, is

$$
\begin{gathered}
\ell_{d t}=\left(\frac{0.016 f_{y} \psi_{e}}{\sqrt{f_{c}^{\prime}}}\right) d_{b} \text { (in.-lb) } \\
\ell_{d t}=\left(\frac{0.19 f_{y} \psi_{e}}{\sqrt{f_{c}^{\prime}}}\right) d_{b}(\mathrm{SI})
\end{gathered}
$$

where $f_{y}$ is the yield strength of the bar (psi or MPa), with an upper limit of $60,000 \mathrm{psi}(420 \mathrm{MPa}) ; f_{c}^{\prime}$ is the compressive strength of concrete (psi or MPa), with an upper limit of $6000 \mathrm{psi}(40 \mathrm{MPa}) ; d_{b}$ is the bar diameter (in. or $\mathrm{mm}$ ); and $\psi_{e}$ the is modification factor for epoxy-coated or zinc and epoxy dual-coated bars. The development length $\ell_{d t}$ may be no less than the larger of $8 d_{b}$ or 6 in. $(150 \mathrm{~mm})$.

To enable comparison of Eq. (1) with test results, the equation is solved for the stress in the headed bar, replacing $\ell_{d t}$ with $\ell_{e h}$, corresponding to the actual embedment length (measured from the critical section to the bearing face of the head). $f_{y}$ is replaced by $f_{s, A C I}$, and $f_{c}^{\prime}$ is replaced by $f_{c m}$, giving

$$
\begin{gathered}
f_{s, A C I}=\frac{\ell_{e h} \sqrt{f_{c m}}}{0.016 d_{b} \psi_{e}} \text { (in.-lb) } \\
f_{s, A C I}=\frac{\ell_{e h} \sqrt{f_{c m}}}{0.19 d_{b} \psi_{e}} \text { (SI) }
\end{gathered}
$$

For a given embedment length, Eq. (2) indicates that the stress developed in a headed bar corresponding to an anchorage failure is proportional to the square root of

ACI Structural Journal, V. 116, No. 3, May 2019

MS No. S-2018-220, doi: 10.14359/51714480, received June 14, 2018, and reviewed under Institute publication policies. Copyright (C) 2019, American Concrete Institute. All rights reserved, including the making of copies unless permission is obtained from the copyright proprietors. Pertinent discussion including author's closure, if any, will be published ten months from this journal's date if the discussion is received within four months of the paper's print publication. 
concrete compressive strength and inversely proportional to the bar diameter. As demonstrated by Shao et al. (2016) and Ghimire et al. (2019), however, the square root of concrete compressive strength overestimates the increase in stress in the bar as the compressive strength increases, and the stress drops more rapidly with increased bar size than characterized by the inverse of the bar diameter. Specifically, Eq. (2) is very conservative in predicting the anchorage strength of headed No. 5 (No. 16) bars but becomes progressively less conservative with increasing bar size and concrete compressive strength.

These observations demonstrate that the current provisions (ACI 318-14) for the development length of headed bars are not applicable over the full range of concrete compressive strengths and bar sizes used in practice. To address these inconsistencies and incorporate key factors affecting the anchorage strength of headed bars including concrete compressive strength, embedment length, bar diameter, spacing between the bars, and confining reinforcement, descriptive equations are developed that accurately characterize the anchorage strength of the headed bars.

\section{RESEARCH SIGNIFICANCE}

Expressions that characterize the anchorage strength of headed bars in concrete are scarce in the literature. Those that are available are either too specific to the tests from which they were developed or do not address the full range of factors affecting anchorage strength. This paper aims to develop descriptive equations for the anchorage strength of headed bars that are applicable over the full range of concrete and steel strengths currently used in construction, along with the other key factors that control anchorage strength.

\section{HEADED BAR DATABASE}

As part of a comprehensive study on the anchorage behavior of headed bars (Shao et al. 2016; Ghimire et al. 2018, 2019), 202 exterior beam-column joint specimens were tested under monotonic loading. The key parameters studied were concrete compressive strength, bar size, embedment length, head size, spacing between the bars, and confining reinforcement within the joint region. Results from 138 out of the 202 specimens, 64 without and 74 with confining reinforcement within the joint region, were used to develop descriptive equations for the anchorage strength of the headed bars. Specimens that provided a wide range of the key parameters affecting the anchorage strength of headed bars were selected. Headed bars without and with confining reinforcement within the joint region were considered. Specimens with bars containing heads with net bearing areas of 3.8 to $9.5 A_{b}\left(A_{b}\right.$ is nominal bar area) and side cover of 2.5 to $4 \mathrm{in}$. (65 to $100 \mathrm{~mm}$ ) were selected as the data set used to develop the descriptive equations. Specimens with large heads $\left(12.9\right.$ to $\left.14.9 A_{b}\right)$ were not included in the development of the equations because, as described by Ghimire et al. (2019), the bars with large heads exhibited higher anchorage strengths than bars with $9.5 A_{b}$ and smaller heads. Some headed bars with short embedment lengths $\ell_{e h}$ relative to the distance from the center of the bar to the top of bearing member tended to exhibit low anchorage strengths and were not used to develop the descriptive equations. As demonstrated by Shao et al. (2016), it is more appropriate to evaluate the latter specimens using strut-and-tie models (ACI 318-14). Two specimens in an early test group had no confining reinforcement above or within the joint region and served as trial specimens to provide information for the design of subsequent beam-column joint specimens. Because the lack of confining reinforcement above the joint is inconsistent with design requirements for reinforced concrete columns (ACI 318-14), these specimens were also not used to develop the descriptive equations. The specimens used in the analysis are listed in Table A.2 of Appendix A. ${ }^{*}$ Specimen details are presented in Table A.3 of Appendix A. The measured concrete compressive strengths $f_{c m}$ ranged from 4050 to 16,030 psi (27.9 to $110.6 \mathrm{MPa}$ ). No. 5, No. 8, and No. 11 (No. 16, No. 25, and No. 36) headed bars with embedment lengths $\ell_{e h}$ ranging from 3.75 to 19.75 in. (95 to $502 \mathrm{~mm}$ ) were tested. Center-to-center spacing between the headed bars, $c_{c h}$, ranged from 2.9 to 11.8 bar diameters $d_{b}$. Friction-forged, taper-threaded, and cold-swaged heads, representing a variety of manufacturing processes, were evaluated. Some coldswaged headed bars included in the analysis contained a threaded coupling sleeve adjacent to the bearing face of the head that exceeded the size of obstructions permitted for HA heads in accordance with ACI 318-14 and ASTM A970-13a. As demonstrated by Shao et al. (2016) and later in this paper, these headed bars exhibited anchorage strengths that were similar to those meeting the provisions in ACI 318-14 and were, thus, included in the data used to develop the equations. Details of the heads are provided in Appendix A and by Ghimire et al. (2018, 2019). Three levels of confining reinforcement placed parallel to the bar within the joint region were studied: no confining reinforcement; two No. 3 hoops; and No. 3 hoops spaced at $3 d_{b}$ (meeting the requirements for a 0.8 reduction factor for the development length calculation of 90-degree hooked bars in accordance with ACI 318-14, allowing comparisons to be made between hooked and headed bars for a constant amount of joint reinforcement). Bar stresses at failure ranged from 33,100 to 153,160 psi (228 to $1056 \mathrm{MPa}$ ). The loading configuration for the beam-column joint specimens is shown in Fig. 1. The headed bars in tension represent the top reinforcement in a beam subjected to negative bending, and the bearing member simulates the compression region of the beam.

\section{ANALYSIS OF TEST RESULTS}

To develop equations that characterize the behavior of headed bars without and with confining reinforcement in simulated beam-column joints, a series of iterative statistical analyses were performed. A least-squares regression technique involving dummy variables was used to determine the effects of key design parameters on the anchorage strength (Draper and Smith 1981). This technique results in a series of parallel lines with different intercepts (Fig. 2) that represent trends in the data. The legends in the figure reflect the order

\footnotetext{
*The Appendix is available at www.concrete.org/publications in PDF format appended to the online version of the published paper. It is also available in hard copy from $\mathrm{ACI}$ headquarters for a fee equal to the cost of reproduction plus handling at the time of the request.
} 


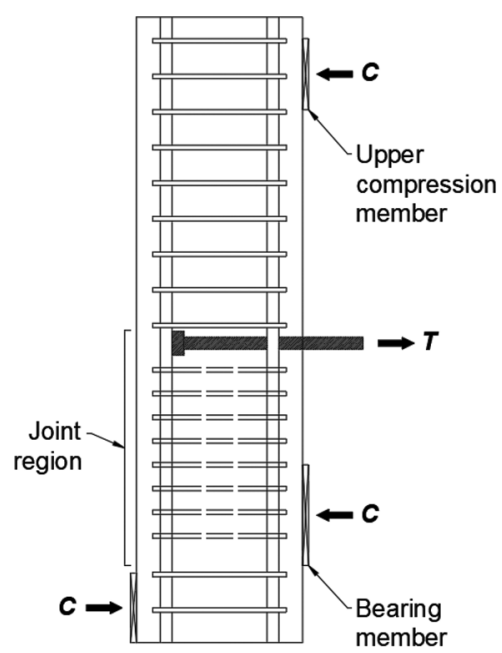

(a)

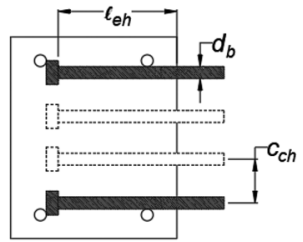

(b)

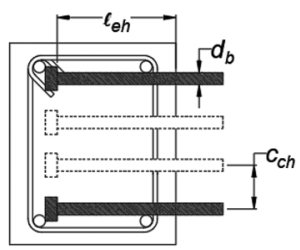

(c)
Fig. 1-Beam-column joint specimens: (a) side view with loading configuration; (b) cross section of specimen at joint region without confining reinforcement; and (c) cross section of specimen at joint region with confining reinforcement parallel to headed bar.

of the data subsets from top to bottom. Descriptive equations of the anchorage strength of headed bars were developed using iterative non-linear regression analyses to ensure a mean test-to-calculated ratio equal to 1.0 while minimizing the sum of the squared differences (1-Test/Calculated $)^{2}$.

\section{Descriptive equations for headed bars without confining reinforcement}

Widely spaced bars-The descriptive equation for the anchorage strength of headed bars without confining reinforcement is based on test results of the 30 specimens with widely spaced bars (center-to-center spacing $\geq 8 d_{b}$ ) and is given by

$$
\begin{gathered}
T_{c}=781 f_{c m}^{0.25} \ell_{e h}{ }^{1.03} d_{b}^{0.35} \text { (in.-lb) } \\
T_{c}=132 f_{c m}{ }^{0.24} \ell_{e h}{ }^{1.03} d_{b}^{0.35}(\mathrm{SI})
\end{gathered}
$$

where $T_{c}$ is the anchorage strength of headed bars without confining reinforcement ( $\mathrm{lb}$ or N); $f_{c m}$ is the concrete compressive strength (psi or $\mathrm{MPa}$ ); $\ell_{e h}$ is the embedment length (in. or $\mathrm{mm}$ ); and $d_{b}$ is the diameter of the headed bar (in. or $\mathrm{mm}$ ).

Figure 2 compares the ratio of test-to-calculated failure load $T / T_{c}$ with the concrete compressive strength for the 30 specimens. $T$ is the average bar force at failure calculated at the peak load on the specimen divided by number of headed bars. In Fig. 2, the dummy variable lines are almost horizontal, indicating that Eq. (3) provides a good match with the test results for concrete strengths between approximately 4000 and $16,000 \mathrm{psi}(27.6$ and $110 \mathrm{MPa})$. The values of $T / T_{c}$ range from 0.80 to 1.18 , with a mean, standard deviation, and coefficient of variation of 1.00, 0.100, and 0.100, respectively.

Equation (3) and Fig. 2 indicate that the anchorage strength of widely spaced headed bars is proportional to the concrete compressive strength to the 0.24 power, rather than

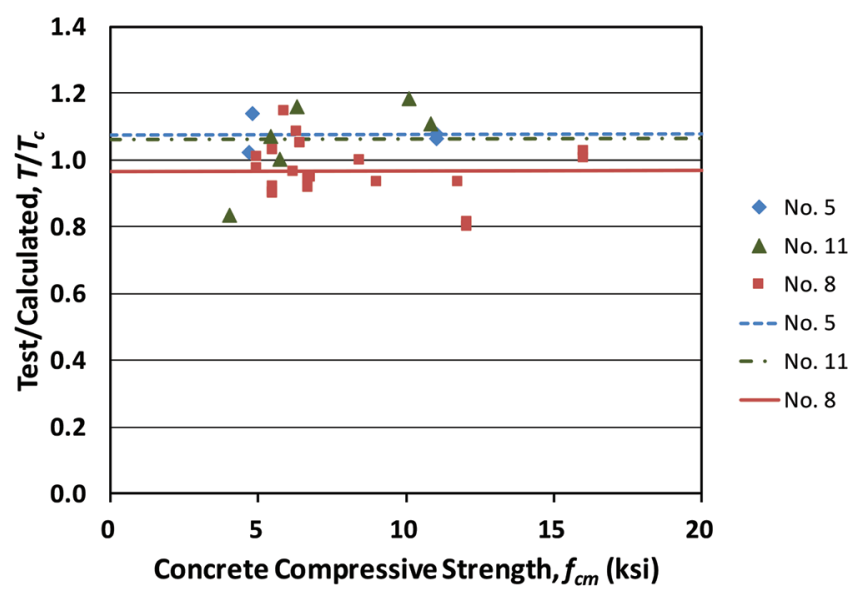

Fig. 2-Ratio of test-to-calculated failure load $\mathrm{T} / \mathrm{T}_{\mathrm{c}}$ versus measured concrete compressive strength $\mathrm{f}_{\mathrm{cm}}$ for specimens with widely spaced bars and no confining reinforcement. (Note: 1 psi $=0.006895$ MPa.)

the 0.5 power of compressive strength, as traditionally used in the ACI Code (ACI 318-14). This observation is consistent with the findings by Darwin et al. $(1996,2005)$ and Zuo and Darwin $(1998,2000)$ on the splice strength of straight bars and by Sperry et al. (2015a,b; 2017) on the anchorage strength of hooked bars, indicating that concrete compressive strength to a power close to $1 / 4$ provides a superior representation of behavior. Justification for the lower power of compressive strength is provided by the fact that both the bond strength of straight reinforcement and the anchorage strength of hooked and headed bars are governed by the combined effects of concrete tensile strength, which controls initial crack formation and increases with the compressive strength to a power between $1 / 2$ and $2 / 3$, and fracture energy, which controls crack propagation and is independent of compressive strength (Darwin et al. 2001). The overall effect is a power between 0.5 and zero.

Equation (3) also indicates that the anchorage strength of headed bars increases almost linearly with the embedment length and that for the same concrete strength and embedment length, larger bars exhibit higher anchorage strengths. The higher strength of larger bars is likely due, at least in part, to the greater effectiveness of the larger heads associated with larger-size bars. The observation that larger headed bars have higher anchorage strengths is also consistent with the observations that larger bars have higher splice strengths than smaller bars for the same splice length (Darwin et al. 1996, 2005; Zuo and Darwin 1998, 2000; Darwin 2005), and larger hooked bars have higher anchorage strengths than smaller hooked bars for the same embedment length (Sperry et al. 2015a,b; 2017).

Closely spaced bars-For the 34 specimens with closely spaced bars (center-to-center spacing $<8 d_{b}$ ), the ratios of anchorage strengths $T / T_{c}$ ( $T_{c}$ based on Eq. (3)) are plotted versus the center-to-center spacing between the bars in Fig. 3. For comparison, the 30 specimens with widely spaced bars used to develop Eq. (3) are also shown in the figure, represented by open symbols.

As shown in Fig. 3, the anchorage strengths of the closely spaced headed bars, regardless of bar size, were generally 


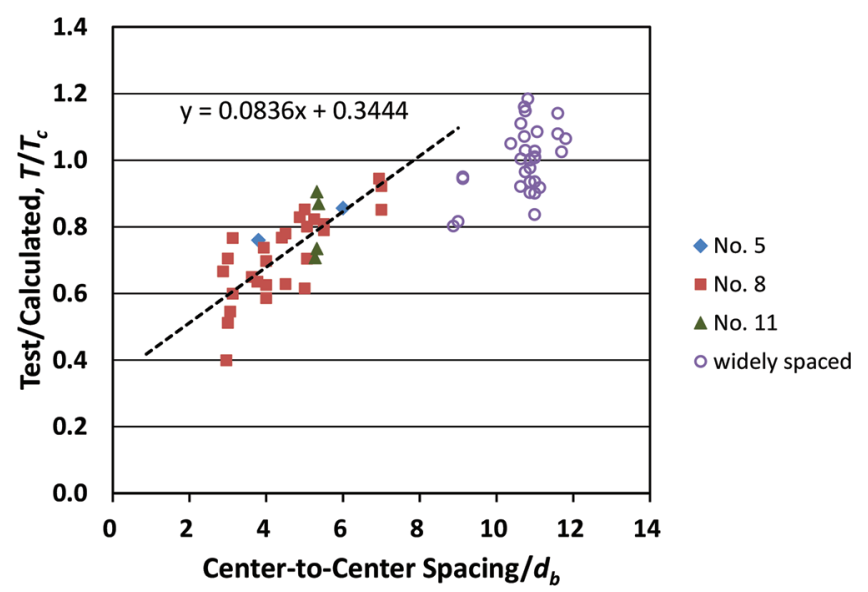

Fig. 3-Ratio of test-to-calculated failure load $\mathrm{T} / \mathrm{T}_{\mathrm{c}}$ versus center-to-center spacing for headed bars without confining reinforcement.

weaker than those of the widely spaced bars. The trend line for the closely spaced bars shows an increase in anchorage strength with an increase in bar spacing. The value of $T / T_{c}$ equals 1.0 at a center-to-center spacing of approximately $8 d_{b}$, which is greater than the value of $6 d_{b}$ observed for closely spaced hooked bars tested in simulated beam-column joint specimens by Ajaam et al. $(2017,2018)$. The difference in critical bar spacing between headed and hooked bars is likely due to the geometry of the head; the larger size of the head relative to the bar reduces the effective clear spacing between heads and may result in interaction between headed bars at slightly greater spacings than was observed for hooked bars. Modifying Eq. (3) to account for the effect of closely spaced headed bars results in Eq. (4)

$$
T_{c}=\left(781 f_{c m}^{0.24} \ell_{e h}^{1.03} d_{b}^{0.35}\right)\left(0.0836 \frac{c_{c h}}{d_{b}}+0.3444\right) \text { (in.-lb) }
$$

with $0.0836\left(c_{c h} / d_{b}\right)+0.3444 \leq 1.0$

$$
T_{c}=\left(132 f_{c m}^{0.24} \ell_{e h}^{1.03} d_{b}^{0.35}\right)\left(0.0836 \frac{c_{c h}}{d_{b}}+0.3444\right)(\mathrm{SI})
$$

with $0.0836\left(c_{c h} / d_{b}\right)+0.3444 \leq 1.0$

where $c_{c h}$ is the center-to-center spacing between the bars (in. or $\mathrm{mm}$ ).

Figure 4 compares the ratio $T / T_{c}$ to the concrete compressive strength for the headed bars (both widely spaced and closely spaced) without confining reinforcement, with $T_{c}$ based on Eq. (4). The slope of the dummy variable lines in the figure indicates that the effect of concrete compressive strength is slightly overestimated by Eq. (4). A slightly lower value for the power of $f_{c m}$ might be more suitable for the combined widely spaced and closely spaced bars, but for simplicity, the 0.24 power for $f_{c m}$ is retained. The ratio $T / T_{c}$ ranges from 0.68 to 1.27 , with a mean, standard deviation, and coefficient of variation of $1.00,0.111$, and 0.111 .

With the descriptive equations (Eq. (3) and (4)) developed for headed bars without confining reinforcement, the ratios of test-to-calculated failure load $T / T_{c}$ are plotted versus

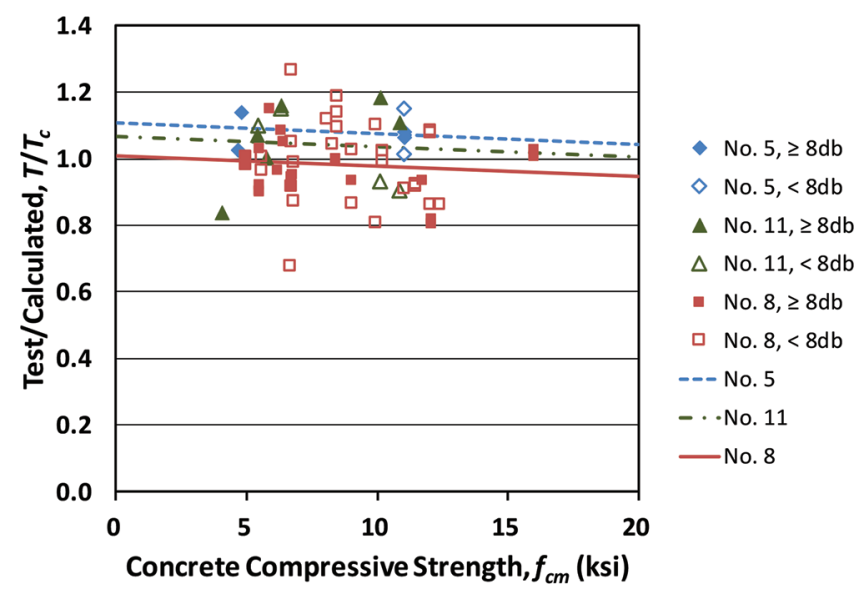

Fig. 4-Ratio of test-to-calculated failure load $\mathrm{T} / \mathrm{T}_{\mathrm{c}}$ versus measured concrete compressive strength $\mathrm{f}_{\mathrm{cm}}$ for specimens without confining reinforcement. (Note: 1 psi $=0.006895$ MPa.)

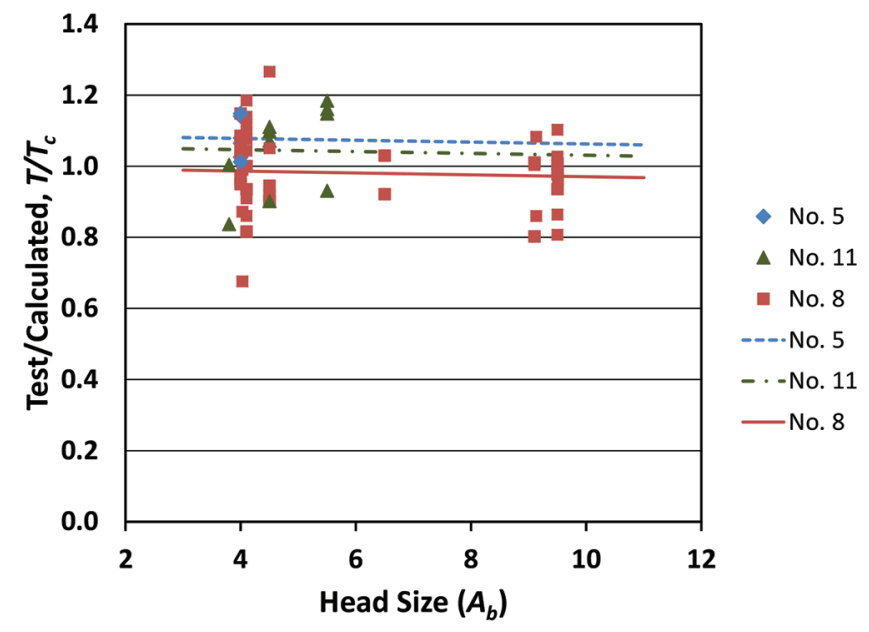

Fig. 5-Ratio of test-to-calculated failure load $\mathrm{T} / \mathrm{T}_{\mathrm{c}}$ versus head size for specimens without confining reinforcement.

head size (net bearing area with respect to bar area) in Fig. 5 for the specimens used to develop the descriptive equations. The slightly negative slope of the trend lines shown in Fig. 5, primarily based on the range of head sizes for No. 8 bars, indicates that the anchorage strength for headed bars without confining reinforcement has a minimal correlation with the head size for heads with bearing areas between 3.8 and $9.5 A_{b}$. This, in turn, justifies grouping headed bars with bearing areas from 3.8 to $9.5 A_{b}$ as a single series for the development of the descriptive equations.

Although not covered in this paper, an analysis of a wider range of specimen types indicates that a modification factor of 0.8 should be applied to $T_{c}$ or $T_{h}$ for headed bars terminating inside a column core (a region of the column cross section located inside the column longitudinal reinforcement) with a clear cover to the bar less than $2.5 \mathrm{in}$. $(64 \mathrm{~mm})$, or terminating in a member other than beam-column joints with clear side cover to the bar less than $8 d_{b}$ (Shao et al. 2016). 


\section{Descriptive equations for headed bars with confining reinforcement}

Widely spaced bars-For the 43 specimens with widely spaced bars and confining reinforcement within the joint region, the development of a descriptive equation is based on two assumptions: 1) the anchorage strength $\left(T_{h}\right)$ is the sum of a concrete contribution $T_{c}$, given by Eq. (3), and a contribution from the confining reinforcement within the joint region $T_{s}$; and 2) the contribution from confining reinforcement is directly related to an effective quantity of confining reinforcement. Confining reinforcement is considered effective if it is fully anchored, in the form of closed hoops, and is located close to the top of the headed bar-within $8 d_{b}$ for No. 3 through No. 8 bars and within $10 d_{b}$ for No. 9 through No. 11 bars. These regions match those observed for hooked bars anchored in simulated beam-column joints (Sperry et al. 2015a,b, 2017).

The second assumption (dealing with the location of the effective confining reinforcement) is supported by the strain measurements in the confining reinforcement (Shao et al. 2016; Ghimire et al. 2018). The results indicate that the hoops located within the appropriate region $\left(8 d_{b}\right.$ or $\left.10 d_{b}\right)$ experienced a significant strain increase at failure (almost all yielded), while the hoops outside this region exhibited much less or a negligible increase in strain. This assumption is also supported by observations of specimens after failure. Most cracks at failure were confined by the hoops that were close to the headed bars, rather than by all the hoops provided in the joint region. Figure 6 shows cracks for a specimen with No. 3 hoops spaced at $3 d_{b}$. The photo was taken after the loose concrete had been removed from the specimen following failure. The specimen contained six hoops within the joint region, but only the top four hoops crossed the major diagonal crack that propagated from the bottom of the head toward the bearing member. The bottom two hoops-one slightly above the top of the bearing member and another slightly below the top of the bearing member (within the compression region) - were below the cracked region.

Based on these assumptions and the analysis of the 43 specimens with confining reinforcement, the contribution of confining reinforcement can be expressed as

$$
\begin{gathered}
T_{s}=48,800 \frac{A_{t t}}{n} d_{b}^{0.88} \text { (in.-lb) } \\
T_{s}=19.5 \frac{A_{t t}}{n} d_{b}^{0.88}(\mathrm{SI})
\end{gathered}
$$

where $T_{s}$ is the contribution of confining reinforcement to the anchorage strength of a headed bar ( $\left(\mathrm{b}\right.$ or N); $A_{t t}$ is the total cross-sectional area of effective confining reinforcement parallel to the headed bars being developed (in. ${ }^{2}$ or $\mathrm{mm}^{2}$ ), which is the product of the cross-sectional area of a single leg of confining reinforcement $\left(A_{t r, l}\right)$ and the total number of single legs of confining reinforcement parallel to the straight portion of a headed bar within $8 d_{b}$ from top of headed bar for No. 3 through No. 8 (No. 10 through No. 25) headed bars and within $10 d_{b}$ from top of headed bar for No. 9 through

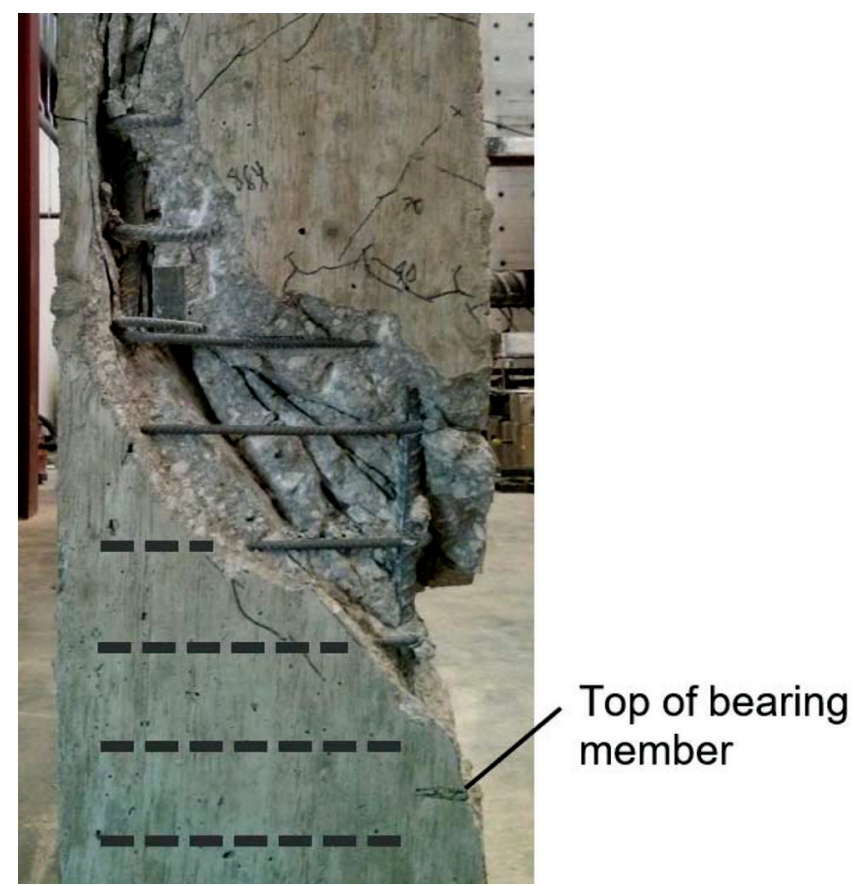

Fig. 6-Cracks confined by effective confining reinforcement (Specimen 11-5-F3.8-6\#3-i-2.5-3-12).

No. 11 (No. 29 through No. 36) headed bars $(N) ; n$ is the number of headed bars; and $d_{b}$ is the diameter of headed bar (in. or $\mathrm{mm}$ ). The term $A_{t t} / n$ is the area of confining reinforcement per headed bar with an upper limit of $0.3 A_{b}$.

Equation (5) indicates that the anchorage strength of headed bars is proportional to the amount of confining reinforcement parallel to the bar and to the bar diameter to the power 0.88 . The fact that $d_{b}$ appears in the equation indicates that a given quantity of confining reinforcement is more effective in increasing the anchorage forces as the size of the headed bar increases. These observations are, in general, consistent with the contribution of confining reinforcement parallel to hooked bars as expressed in Eq. (6) (Sperry et al. 2017). The power of 1.06 on the confining reinforcement term $A_{t h} / n$ is close to 1.0 , signifying a linear relationship between the anchorage strength of hooked bars $\left(T_{s}\right)$ and confining reinforcement

$$
\begin{gathered}
T_{s}=54,250\left(\frac{A_{t h}}{n}\right)^{1.06} d_{b}^{0.59} \text { (in.-lb) } \\
T_{s}=37.6\left(\frac{A_{t h}}{n}\right)^{1.06} d_{b}^{0.59}(\mathrm{SI})
\end{gathered}
$$

where $A_{t h}$ is the total cross-sectional area of effective confining reinforcement parallel to the hooked bars being developed (in. ${ }^{2}$ or $\mathrm{mm}^{2}$ ), analogous to $A_{t t}$ for headed bars.

Combining Eq. (3) with Eq. (5), the anchorage strength for widely spaced headed bars with confining reinforcement can be expressed as

$$
T_{h}=781 f_{c m}^{0.24} \ell_{e h}^{1.03} d_{b}^{0.35}+48,800 \frac{A_{t t}}{n} d_{b}^{0.88} \text { (in.-lb) }
$$




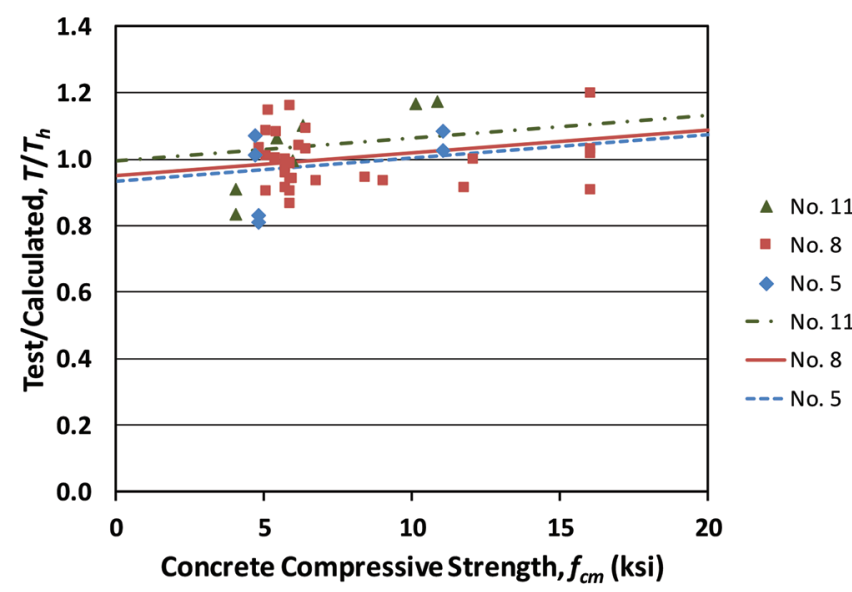

Fig. 7-Ratio of test-to-calculated failure load $\mathrm{T} / \mathrm{T}_{\mathrm{h}}$ versus measured concrete compressive strength $\mathrm{f}_{\mathrm{cm}}$ for specimens with widely-spaced bars and confining reinforcement. (Note: 1 psi $=0.006895$ MPa.)

$$
T_{h}=132.1 f_{c m}^{0.24} \ell_{e h}^{1.03} d_{b}^{0.35}+19.5 \frac{A_{t t}}{n} d_{b}^{0.88} \text { (SI) }
$$

Figure 7 compares the ratio $T / T_{h}$ to the concrete compressive strength $f_{c m}$ for the 43 specimens. $T$ is the average peak load. $T_{h}$ is based on Eq. (7). The slope of dummy variable lines in the figure indicates that the effect of concrete compressive strength is slightly underestimated by Eq. (7). The values of $T / T_{h}$ range from 0.81 to 1.20 , with a mean, standard deviation, and coefficient of variation of 1.00 , 0.095 , and 0.095 , respectively.

Closely spaced bars-For the 31 specimens with closely spaced bars and confining reinforcement, the ratios of anchorage strengths $T / T_{h}$, with $T_{h}$ based on Eq. (7), are plotted versus the center-to-center spacing between the bars in Fig. 8. The 43 specimens with widely spaced headed bars used to develop Eq. (7) are also shown in the figure, represented by open symbols.

Like the closely spaced headed bars without confining reinforcement, Fig. 8 shows that the closely spaced bars with confining reinforcement generally exhibited lower anchorage strengths than the widely spaced bars. The trend line, however, is flatter than that shown in Fig. 3, indicating that the anchorage strength of headed bars with confining reinforcement is affected less by close bar spacing than that of headed bars without confining reinforcement. Of the 31 specimens with closely spaced bars and confining reinforcement, 14 had two No. 3 hoops and 17 had No. 3 hoops at $3 d_{b}$. Based on the trend line shown in Fig. 8, the anchorage strength for both closely and widely spaced headed bars with confining reinforcement can be expressed as

$$
T_{h}=\left(781 f_{c m}^{0.24} \ell_{e h}^{1.03} d_{b}^{0.35}+48,800 \frac{A_{t t}}{n} d_{b}^{0.88}\right)\left(0.0622 \frac{c_{c h}}{d_{b}}+0.5428\right) \text { (in.-lb) }
$$

$$
T_{h}=\left(132 f_{c m}^{0.24} \ell_{e h}{ }^{1.03} d_{b}^{0.35}+19.5 \frac{A_{t t}}{n} d_{b}^{0.88}\right)\left(0.0622 \frac{c_{c h}}{d_{b}}+0.5428\right)(\mathrm{SI})
$$

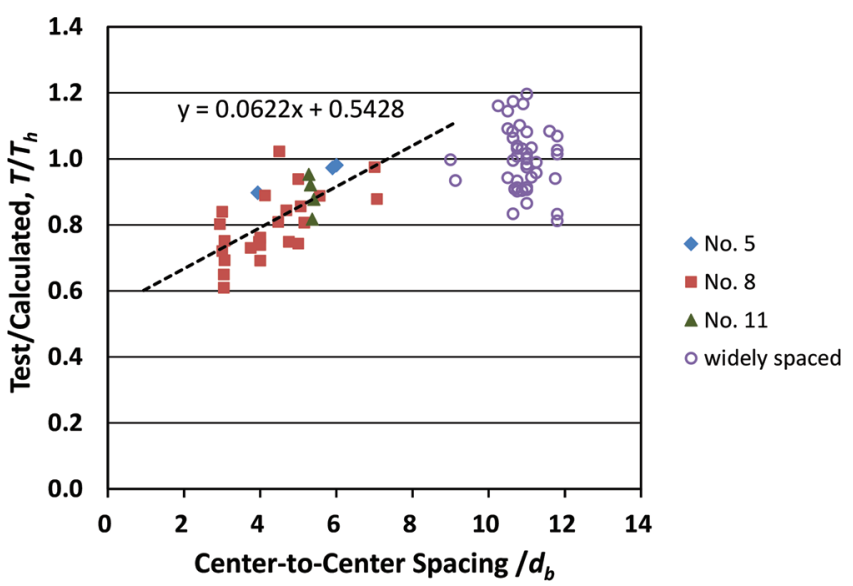

Fig. 8-Ratio of test-to-calculated failure load $\mathrm{T} / \mathrm{T}_{\mathrm{h}}$ versus center-to-center spacing for headed bars with confining reinforcement.

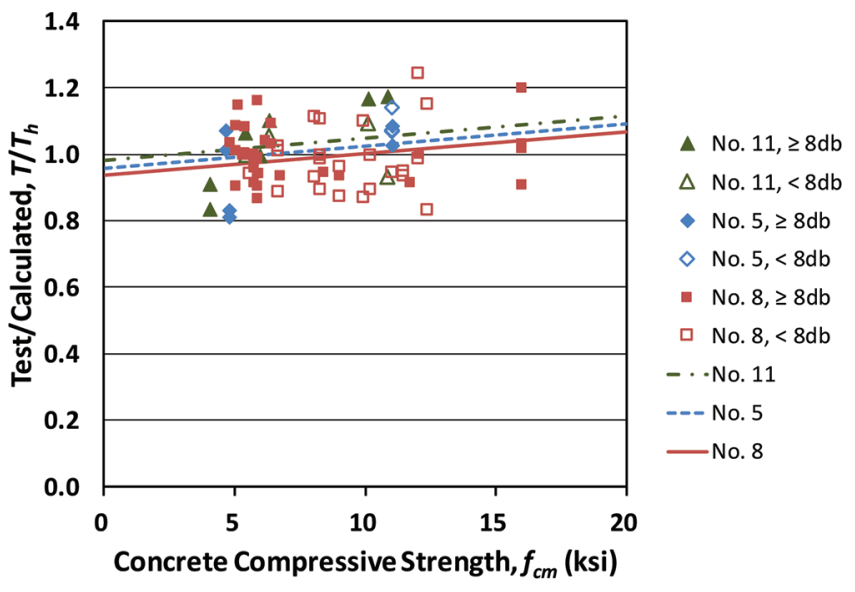

Fig. 9-Ratio of test-to-calculated load $\mathrm{T} / \mathrm{T}_{\mathrm{h}}$ versus measured concrete compressive strength $\mathrm{f}_{\mathrm{cm}}$ for specimens with confining reinforcement. (Note: 1 psi $=0.006895 \mathrm{MPa}$.)

with $0.0622\left(c_{c h} / d_{b}\right)+0.5428 \leq 1.0$ and $A_{t t} / n \leq 0.3 A_{b}$.

Figure 9 compares the ratio $T / T_{h}$ with concrete compressive strength for the headed bars (both widely spaced and closely spaced) with confining reinforcement, with $T_{h}$ based on Eq. (8). The slope of dummy variable lines in the figure indicates that the effect of concrete compressive strength is slightly underestimated by Eq. (8). The ratio $T / T_{h}$ ranges from 0.81 to 1.24 , with a mean, standard deviation, and coefficient of variation of $1.00,0.095$, and 0.095 , respectively.

With the descriptive equation (Eq. (8)) developed for headed bars with confining reinforcement, the ratios of test-to-calculated failure load $T / T_{h}$ are plotted versus head size in Fig. 10 for the specimens used to develop the descriptive equations.

The closely spaced, slightly upward trend lines shown in Fig. 10 indicate that within a range of 3.8 to $9.5 A_{b}$, head size has a minimal influence on the anchorage strength for headed bars with confining reinforcement. As was found for specimens without confining reinforcement, this result justifies treating headed bars with 3.8 to $9.5 A_{b}$ heads as a single series for the development of the descriptive equations.

In Fig. 11, the failure loads $T$ for all specimens are compared with the values of calculated failure load $T_{h}$ 


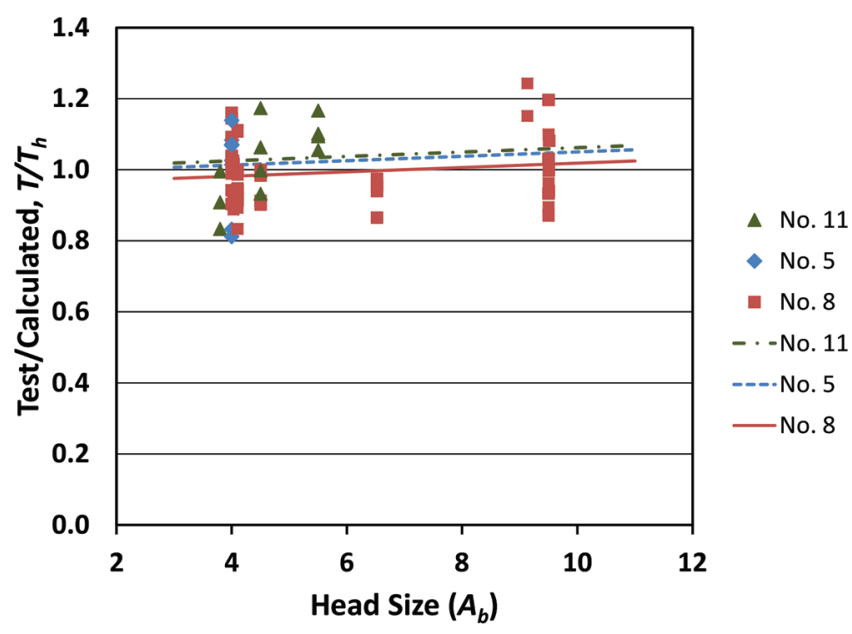

Fig. 10-Ratio of test-to-calculated failure load $\mathrm{T} / \mathrm{T}_{\mathrm{h}}$ versus head size for specimens with confining reinforcement.

obtained using the descriptive equations, Eq. (4) and (8). As shown in the figure, the descriptive equations slightly overestimate the anchorage strength for No. 5 bars and slightly underestimate the anchorage strength for No. 11 bars. The variation, however, is small given the range of data.

\section{Headed bars with large obstructions}

The anchorage strengths of headed bars with cold-swaged threaded coupling sleeves with obstructions exceeding the dimensional limits for HA heads in ASTM A970-13a (heads permitted by ACI 318-14) O4.5 and O9.1, were compared with the anchorage strengths of the headed bars meeting the requirements. The net bearing area of the large obstruction heads is taken as the difference between the gross area of head and the area of the obstruction adjacent to the head. The dimensions of the heads and obstructions are shown in Appendix A. Twenty-two specimens contained headed bars with large obstructions with net bearing areas of 4.5 and $9.1 A_{b}$. Of the 22 specimens, 14 had no confining reinforcement and eight had confining reinforcement within the joint region parallel to the headed bar. For the 14 specimens without confining reinforcement, the ratio of test-tocalculated failure load $T / T_{c}$ ranged from 0.90 to 1.27 , with a mean, standard deviation, and coefficient of variation of $1.01,0.107$, and 0.106 , respectively. For the remaining eight specimens with confining reinforcement, the ratio of test-to-calculated failure load $T / T_{h}$ ranged from 0.90 to 1.17 , with a mean, standard deviation, and coefficient of variation of $0.99,0.089$, and 0.090 , respectively. This indicates that the headed bars with large obstructions have similar anchorage strengths to those that met the requirements of HA heads in ASTM A970-13a and can be safely used in design. These observations were used to modify the criteria for the size of obstructions and are now permitted for HA heads by ASTM A970-18.

\section{SUMMARY AND CONCLUSIONS}

Descriptive equations for the anchorage strength of headed bars in concrete were developed. The descriptive equations account for the effects of concrete compressive strength, embedment length, bar size, spacing between the

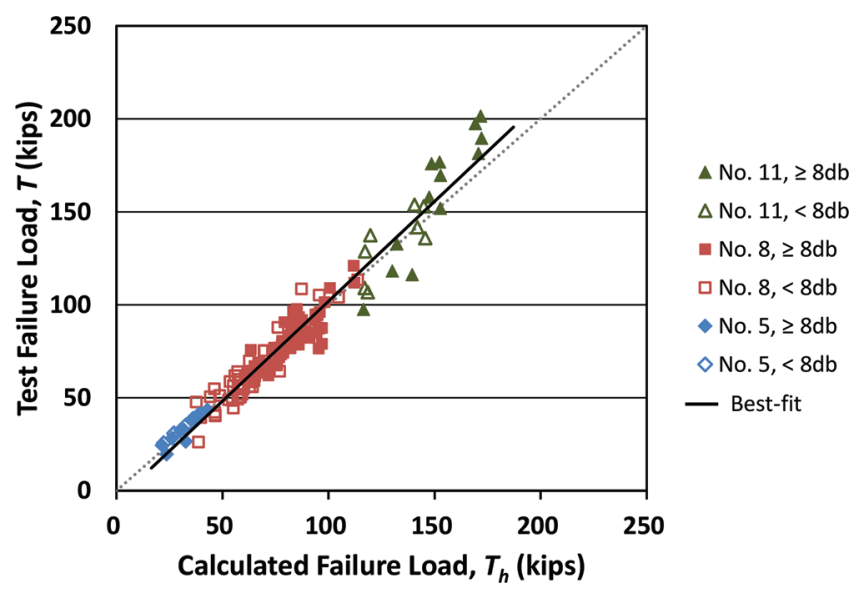

Fig. 11-Test failure load $\mathrm{T}$ versus calculated failure load $\mathrm{T}_{\mathrm{h}}$ for specimens without and with confining reinforcement. $\mathrm{T}_{\mathrm{h}}$ is calculated based on Eq. (4) and (8). (Note: $1 \mathrm{kip}=4.448 \mathrm{kN}$.)

bars, and confining reinforcement. Results from 138 beamcolumn joint specimens, 64 without and 74 with confining reinforcement within the joint region, were used to develop the equations. No. 5, No. 8, and No. 11 (No. 16, No. 25, and No. 36) headed bars with embedment lengths ranging from 3.75 to $19.75 \mathrm{in}$. ( 95 to $502 \mathrm{~mm}$ ) were tested in concrete compressive strengths ranging from 4050 to 16,030 psi (27.9 to $110.6 \mathrm{MPa}$ ). Center-to-center spacing between the headed bars ranged from 2.9 to 11.8 bar diameters. Bar stresses at failure ranged from 33,100 to $153,160 \mathrm{psi}$ (228 to $1056 \mathrm{MPa})$. Some headed bars contained obstructions adjacent to the head that exceeded the dimensions permitted for HA heads in ACI 318-14 and ASTM A970-13a but are now permitted by ASTM A970-18.

The following conclusions are drawn based on the results presented in this paper.

1. The effect of concrete compressive strength on the anchorage strength of headed bars can be represented by the compressive strength to the 0.24 power, which is close to the relationship for the development length of straight bars and the anchorage strength of hooked bars.

2. Anchorage strength is improved by the addition of confining reinforcement parallel to the headed bar, a factor that is not included in current design provisions; the increase in anchorage strength is proportional to the amount of confining reinforcement per headed bar within eight to 10 bar diameters of the headed bar.

3. For a given embedment length, the anchorage strength of headed bars increases with an increase in bar diameter.

4. The anchorage strength of headed bars begins to decrease as the center-to-center spacing between the bars decreases below eight bar diameters, another factor not included in current design provisions.

5. Headed bars with obstructions larger than permitted in ACI 318-14 and ASTM A970-13a that meet the new provisions in ASTM A970-18 exhibit similar anchorage strengths to those that meet the provisions in ACI 318-14.

\section{AUTHOR BIOS}

ACI member Krishna P. Ghimire is a Teaching Assistant Professor of Civil Engineering at Kansas State University, Manhattan, KS. He received his BS 
from Tribhuvan University, Kirtipur, Nepal, and his MS and PhD from the University of Kansas, Lawrence, KS. He is a member of Joint ACI-ASCE Committee 408, Bond and Development of Steel Reinforcement.

Yun Shao is a Staff Professional with Kleinfelder, San Diego, CA. She received her BS and MS from Southeast University, Nanjing, China, and her PhD from the University of Kansas.

ACI Honorary Member David Darwin is the Deane E. Ackers Distinguished Professor and Chair of the Department of Civil, Environmental, and Architectural Engineering at the University of Kansas and a Past President of ACI. He is a member of ACI Committees 222, Corrosion of Metals in Concrete; 224, Cracking; Subcommittee 318-B, Anchorage and Reinforcement (Structural Concrete Building Code); and Joint ACI-ASCE Committees 408, Bond and Development of Steel Reinforcement; 445, Shear and Torsion; and 446, Fracture Mechanics of Concrete.

ACI member Matthew O'Reilly is a Chair's Council Assistant Professor of Civil, Environmental, and Architectural Engineering at the University of Kansas. He received his $B S$ in mechanical engineering from the University of Rochester, Rochester, NY, and his MS and PhD in civil engineering from the University of Kansas. He is a member of ACI Committees 123, Research and Current Developments; 222, Corrosion of Metals in Concrete; and Joint ACI-ASCE Committee 408, Bond and Development of Steel Reinforcement.

\section{ACKNOWLEDGMENTS}

Support for the study was provided by the Electric Power Research Institute, Concrete Reinforcing Steel Institute Education and Research Foundation, BarSplice Products, Inc., Headed Reinforcement Corp., and LENTON ${ }^{\circledR}$ products from Pentair ${ }^{\mathbb{R}}$. Additional materials were supplied by Commercial Metals Company, Gerdau Corporation, Nucor Corporation, MMFX Technologies Corporation, Dayton Superior, Midwest Concrete Materials, and Grace Construction Products. Thanks are due to K. Barry, M. Ruis, and D. Scott, who provided project oversight for the Advanced Nuclear Technology Program of the Electric Power Research Institute; and to N. Anderson, C. Kopczynski, M. Mota, J. Munshi, and C. Paulson, who served as industry advisors.

\section{NOTATION}

$A_{b r g}=$ net bearing area of head

$A_{\text {th }}=$ total cross-sectional area of effective confining reinforcement within joint region for hooked bars

$A_{t r, l}=$ cross-sectional area of single leg of confining reinforcement

$A_{t t}=$ total cross-sectional area of effective confining reinforcement within joint region for headed bars $\left(N A_{t r, l}\right)$

$d_{b}=$ nominal diameter of headed bar

$f_{c}^{\prime}=$ specified concrete compressive strength

$f_{c m}=$ measured average concrete compressive strength

$f_{s, A C I}=$ stress in headed bar as calculated by Section 25.4.4.2 of ACI 318-14

$f_{y}=$ yield strength of headed bar

$\ell_{d t}=$ development length in tension of headed deformed bar, measured from critical section to bearing face of the head

$\ell_{e h}=$ embedment length measured from front face of column to bearing face of the head

$N=$ number of legs of confining reinforcement parallel to straight portion of headed bar within $8 d_{b}$ from top of headed bar for No. 3 through No. 8 (No. 10 through No. 25) headed bars or $10 d_{b}$ from top of headed bar for No. 9 through No. 11 (No. 29 through No. 36) headed bars

$n=$ number of headed bars confined by $N$ legs

$T=$ average bar force at failure on headed bars in a specimen (failure load)

$T_{c}=$ contribution of concrete to headed bar anchorage strength

$T_{h}=$ headed bar anchorage strength

$T_{s}=$ contribution of confining reinforcement oriented parallel to hooked or headed bar to anchorage strength

$\psi_{e}=$ factor used to modify development length based on reinforcement coating as defined in ACI 318-14 Section 25.4.4.3

\section{REFERENCES}

ACI Committee 318, 2008, "Building Code Requirements for Structural Concrete (ACI 318-08) and Commentary (ACI 318R-08)," American Concrete Institute, Farmington Hills, MI, 465 pp.
ACI Committee 318, 2014, "Building Code Requirements for Structural Concrete (ACI 318-14) and Commentary (ACI 318R-14)," American Concrete Institute, Farmington Hills, MI, 520 pp.

Ajaam, A.; Darwin, D.; and O’Reilly, M. O., 2017, “Anchorage Strength Reinforcing Bars with Standard Hooks," SM Report No. 125, University of Kansas Center for Research, Lawrence, Kansas, Apr., 346 pp.

Ajaam, A.; Yasso, S.; Darwin, D.; O’Reilly, M.; and Sperry, J., 2018, "Anchorage Strength of Closely Spaced Hooked Bars," ACI Structural Journal, V. 115, No. 4, July, pp. 1143-1152.

ASTM A970/A970M-13a, 2013, "Standard Specification for Headed Steel Bars for Concrete Reinforcement," ASTM International, West Conshohocken, PA, 9 pp.

ASTM A970/A970M-18, 2018, "Standard Specification for Headed Steel Bars for Concrete Reinforcement," ASTM International, West Conshohocken, PA, 10 pp.

Darwin, D., 2005, “Tension Development Length and Lap Splice Design for Reinforced Concrete Members," Progress in Structural Engineering and Materials, V. 7, No. 4, Oct.-Dec., pp. 210-225. doi: 10.1002/pse.206

Darwin, D.; Barham, S.; Kozul, R.; and Luan, S., 2001, "Fracture Energy of High-Strength Concrete," ACI Structural Journal, V. 98, No. 5, Sept.-Oct., pp. 410-417.

Darwin, D.; Lutz, L. A.; and Zuo, J., 2005, "Recommended Provisions and Commentary on Development and Lap Splice Lengths for Deformed Reinforcing Bars in Tension," ACI Structural Journal, V. 102, No. 6, Nov.-Dec., pp. 892-900.

Darwin, D.; Zuo, J.; Tholen, M. L.; and Idun, E. K., 1996, "Development Length Criteria for Conventional and High Relative Rib Area Reinforcing Bars," ACI Structural Journal, V. 93, No. 3, May-June, pp. 1-13.

DeVries, R. A.; Jirsa, J. O.; and Bashandy, T., 1998, "Effects of Transverse Reinforcement and Bonded length on the Side-Blowout Capacity of Headed Reinforcement," Recent Developments in the Design and Specification of Concrete Paving Systems, SP-180, D. G. Zollinger, ed., American Concrete Institute, Farmington Hills, MI, Oct., pp. 367-390.

DeVries, R. A.; Jirsa, J. O.; and Bashandy, T., 1999, “Anchorage Capacity in Concrete of Headed Reinforcement with Shallow Embedments," $A C I$ Structural Journal, V. 96, No. 5, Sept.-Oct., pp. 728-737.

Draper, N. R., and Smith, H., 1981, Applied Regression Analysis, second edition, John Wiley and Sons, New York, pp. 241-249.

Ghimire, K. P.; Darwin, D.; and O'Reilly, M., 2018, "Anchorage of Headed Reinforcing Bars in Concrete," SM Report No. 127, University of Kansas Center for Research, Lawrence, KS, Jan., 278 pp.

Ghimire, K. P.; Shao, Y.; Darwin, D.; and O’Reilly, M., 2019, “Conventional and High-Strength Headed Bars-Part 1: Anchorage Test,' ACI Structural Journal, V. 116, No. 3, May, pp. 257-266. doi: 10.14359/51714479.

Shao, Y.; Darwin, D.; O’Reilly, M.; Lequesne, R. D.; Ghimire, K.; and Hano, M., 2016, "Anchorage of Conventional and High-Strength Headed Reinforcing Bars," SM Report No. 117, University of Kansas Center for Research, Lawrence, KS, Aug., 234 pp.

Sperry, J.; Al-Yasso, S.; Searle, N.; DeRubeis, M.; Darwin, D.; O’Reilly, M.; Matamoros, A.; Feldman, L.; Lepage, A.; Lequesne, R.; and Ajaam, A., 2015a, "Anchorage of High-Strength Reinforcing Bars with Standard Hooks," SM Report No. 111, University of Kansas Center for Research, Lawrence, Kansas, June, 243 pp.

Sperry, J.; Darwin, D.; O'Reilly, M.; and Lequesne, R., 2015b, "Anchorage Strength of Conventional and High-Strength Hooked Bars in Concrete," SM Report No. 115, University of Kansas Center for Research, Lawrence, Kansas, Dec., 266 pp.

Sperry, J.; Darwin, D.; O’Reilly, M.; Matamoros, A.; Feldman, L.; Lepage, A.; Lequesne, R.; and Yasso, S., 2017, "Conventional and HighStrength Hooked Bars-Part 2: Data Analysis," ACI Structural Journal, V. 114, No. 1, Jan.-Feb., pp. 267-276. doi: 10.14359/51689457

Thompson, M. K.; Jirsa, J. O.; and Breen, J. E., 2006a, "CCT Nodes Anchored by Headed Bars-Part 2: Capacity of Nodes," ACI Structural Journal, V. 103, No. 1, Jan.-Feb., pp. 65-73.

Thompson, M. K.; Ledesma, A.; Jirsa, J. O.; and Breen, J. E., 2006b, "Lap Splices Anchored by Headed Bars," ACI Structural Journal, V. 103, No. 2, Mar.-Apr., pp. 271-279.

Thompson, M. K.; Ziehl, M. J.; Jirsa, J. O.; and Breen, J. E., 2005, “CCT Nodes Anchored by Headed Bars-Part 1: Behavior of Nodes," ACI Structural Journal, V. 102, No. 6, Nov.-Dec., pp. 808-815.

Zuo, J., and Darwin, D., 1998, "Bond Strength of High Relative Rib Area Reinforcing Bars," SM Report No. 46, University of Kansas Center for Research, Lawrence, KS, Jan., $350 \mathrm{pp}$.

Zuo, J., and Darwin, D., 2000, "Splice Strength of Conventional and High Relative Rib Area Bars in Normal and High Strength Concrete," $A C I$ Structural Journal, V. 97, No. 4, July-Aug., pp. 630-641. 


\section{APPENDIX A: NOTATION, CONVERSION FACTORS, HEAD DETAILS, REINFORCEMENT LAYOUTS, AND DATA TABLES}

\section{A.1 NOTATION AND CONVERSION FACTORS}

\section{Notation}

$A_{a b} \quad$ Total cross-sectional area of all confining reinforcement parallel to $\ell d t$ for headed bars being developed in beam-column joints and located within $8 d_{b}$ of the bottom (top) of the headed bars in direction of the outside of the joint for No. 3 through No. 8 headed bars or within $10 d_{b}$ of the bottom (top) of the bar in direction of the outside of the joint for No. 9 through No. 11 headed bars

$A_{b} \quad$ Area of an individual headed bar

$A_{b r g} \quad$ Net bearing area of the head of headed deformed bar

$A_{h s} \quad$ Total cross-sectional area of headed bars being developed

$A_{t r, l} \quad$ Area of single leg of confining reinforcement within joint region

$A_{t t} \quad$ Total cross-sectional area of all confining reinforcement parallel to $\ell_{d t}$ for headed bars being developed in beam-column joints and located within $8 d_{b}$ of the top (bottom) of the headed bars in direction of the interior of the joint for No. 3 through No. 8 headed bars or within $10 d_{b}$ of the top (bottom) of the bar in direction of the interior of the joint for No. 9 through No. 11 headed bars; or minimum total cross-sectional area of all confining reinforcement parallel to headed bars being developed in members other than beam-column joints within $7 \frac{1}{2} d_{b}$ on one side of the bar centerline for No. 3 through No. 8 headed bars or within $91 / 2 d_{b}$ on one side of the bar centerline for No. 9 through No. 11 headed bars

$b \quad$ Width of column

$c_{b c} \quad$ Clear cover measured from the back of the head to the back of the member

$c_{h} \quad$ Clear spacing between adjacent headed bars

$c_{o} \quad$ Clear cover measured from the head to the side of the column

$c_{s b} \quad$ Clear cover measured from the bottom of the beam to the headed bar

$c_{s o} \quad$ Clear cover measured from the side of the headed bar to the side of the member

$c_{\text {so,avg }}$ Average clear side cover of the headed bars

$d_{b} \quad$ Nominal diameter of bar

$d_{\text {eff }} \quad$ Effective value of $d$ for beam-column joint

$d_{t r} \quad$ Nominal bar diameter of confining reinforcement within joint region

$d_{\text {tro }} \quad$ Nominal bar diameter of confining reinforcement outside joint region

$f_{c m} \quad$ Measured concrete compressive strength

$f_{s u} \quad$ Average stress in headed bars at failure

$f_{s u \text {,max }}$ Maximum stress in individual headed bar

$h \quad$ Depth of column

$h_{c l} \quad$ Height measured from the center of the headed bar to the top of the bearing member

$\ell_{d t} \quad$ Development length in tension of headed deformed bar, measured from the critical section to the bearing face of the head

$\ell_{\text {eh }} \quad$ Embedment length measured from the bearing face of the head to the face of the member

$\ell_{\text {eh,avg }}$ Average embedment length of headed bars

$n \quad$ Number of headed bars loaded simultaneously

$N \quad$ Number of legs of confining reinforcement in joint region

$T \quad$ Test failure load on a headed bar; average load on headed bars at failure

$T_{c} \quad$ Contribution of concrete to headed bar anchorage strength calculated using Eq. (3) or (4)

$T_{h} \quad$ Headed bar anchorage strength calculated using Eq. (7) or (8) 
$T_{\text {ind }} \quad$ Peak load on individual headed bar at failure

$T_{\max } \quad$ Maximum load on individual headed bar

$T_{\text {total }} \quad$ Sum of loads on headed bars at failure

$c_{c h} \quad$ Center-to-center spacing between adjacent headed bars

$S_{\text {tr }} \quad$ Center-to-center spacing of confining reinforcement (hoops) within joint region

Stro Center-to-center spacing of hoops outside joint region

Failure types

CB Concrete breakout

SB Side blowout

FP Local front pullout (secondary failure)

BS Back cover spalling (secondary failure)

Y Yield of headed bars

Specimen identification

(A@B) C-D-E-F\#G-H-I-J-K

A Number of headed bars in the specimen

B Center-to-center spacing between headed bars in terms of bar diameter

(A@B = blank, indicates specimens with 2 headed bars)

C ASTM in.-lb bar size

D Nominal compressive strength of concrete

E Head type

F Number of bars used as confining reinforcement within the joint region

G ASTM in.-lb bar size of confining reinforcement (if $\mathrm{F} \# \mathrm{G}=0=$ no confining reinforcement)

$\mathrm{H} \quad$ Hooked bars placed inside (i) or outside (o) of longitudinal reinforcement

I Nominal value of $c_{s o}$

$\mathrm{J} \quad$ Nominal value of $c_{b c}$

K Nominal value of $\ell_{\text {eh }}$

\section{Conversion Factors}

1 in. $=25.4 \mathrm{~mm}$

1 kip $=4.448 \mathrm{kN}$

$1 \mathrm{ksi}=6.895 \mathrm{MPa}$

$1 \mathrm{psi}=0.006895 \mathrm{MPa}$

Bar sizes: No. 3 (No. 10), No. 4 (No. 13), No. 5 (No. 16), No. 6 (No. 19), No. 7 (No. 22), No. 8

(No. 25), No. 9 (29), No. 10 (No. 32), No. 11 (No. 36) 


\section{A.2 DETAILS OF HEADS}

Table A.1 Details of friction-forged headed bars

\begin{tabular}{|c|c|c|c|c|c|c|c|c|c|}
\hline & Dimension Notation & Designation & $\begin{array}{l}\text { Bar } \\
\text { Size }\end{array}$ & $\begin{array}{c}b \\
\text { (in.) }\end{array}$ & $\begin{array}{c}h \\
\text { (in.) }\end{array}$ & $\begin{array}{c}t \\
\text { (in.) }\end{array}$ & $\begin{array}{l}d_{o b s} \\
\text { (in.) }{ }^{[1]}\end{array}$ & $\begin{array}{c}t_{o b s} \\
\text { (in.) }{ }^{[1]}\end{array}$ & $\begin{array}{c}\text { Net } \\
\text { Bearing } \\
\text { Area } \\
\left(A_{b r g}\right)^{[2]}\end{array}$ \\
\hline \multirow{6}{*}{ 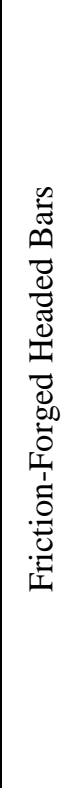 } & \multirow{3}{*}{$h$} & F4.0 & No. 5 & 1.25 & 1.25 & 0.5 & 0.93 & 0.46 & $4.1 A_{b}$ \\
\hline & & F4.1 ${ }^{[3]}$ & No. 8 & 2.02 & 2 & 1 & 1.54 & 0.60 & $4.1 A_{b}$ \\
\hline & & F3.8 & No. 11 & 2.5 & 3 & 1.375 & 2.11 & 0.73 & $3.8 A_{b}$ \\
\hline & \multirow{3}{*}{$b \times t$} & F13.1 & No. 5 & 1.25 & 3.5 & 0.5 & 0.93 & 0.46 & $13.1 A_{b}$ \\
\hline & & F9.1 ${ }^{[3]}$ & No. 8 & 2.03 & 3.98 & 1.02 & 1.58 & 0.62 & $9.2 A_{b}$ \\
\hline & & F8.6 & No. 11 & 2.5 & 6 & 1.375 & 2.11 & 0.73 & $8.6 A_{b}$ \\
\hline
\end{tabular}

[1] See Fig. A1

[2] Net bearing area calculated as gross head area minus bar area. These heads contained obstructions adjacent to the bearing face of the head, as shown in Fig. A1, A3b, and A3c. These obstructions, however, did not have any detrimental effects on the anchorage strength of the headed bars and, therefore, are not considered to detract from the net bearing area of the head (Ghimire et al. 2018).

[3] Head dimensions updated from those given by Shao et al. (2016) (Ghimire et al. 2018)

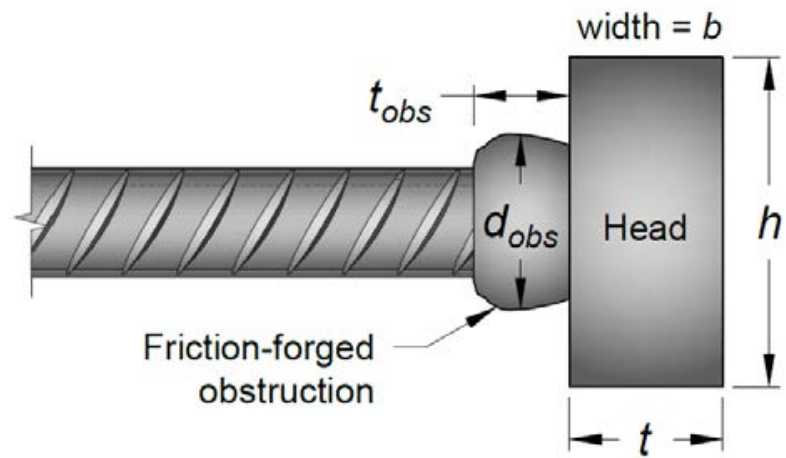

Fig. A1 Friction-forged obstruction adjacent to the bearing face of the head 
Table A.1 Cont. Details of taper-threaded and cold-swaged headed bars

\begin{tabular}{|c|c|c|c|c|c|c|}
\hline & Dimension Notation & Designation & $\begin{array}{l}\text { Bar } \\
\text { Size }\end{array}$ & $d$ (in.) & $t$ (in.) & $\begin{array}{l}\text { Net Bearing } \\
\text { Area }\left(A_{b r g}\right)^{[1]}\end{array}$ \\
\hline \multirow{2}{*}{ 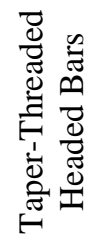 } & & $\mathrm{T} 4.0$ & No. 8 & 2.25 & 1.5 & $4.0 A_{b}$ \\
\hline & & T9.5 & No. 8 & 3.25 & 1.5 & $9.5 A_{b}$ \\
\hline \multirow{5}{*}{ 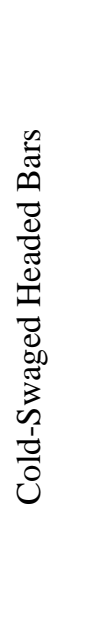 } & & S4.0 [2] [3] & No. 6 & 1.63 & 1.52 & $4.1 A_{b}$ \\
\hline & & S6. $5^{[2][3]}$ & No. 8 & 2.4 & 1.78 & $5.0 A_{b}$ \\
\hline & & S5.5 [3] & No. 11 & 3.5 & 2.75 & $5.5 A_{b}$ \\
\hline & & S9.5 & No. 8 & 3.25 & 2.75 & $9.5 A_{b}$ \\
\hline & & S14.9 [2] & No. 8 & 4.01 & 2.66 & $15 A_{b}$ \\
\hline
\end{tabular}

[1] Net bearing area calculated as gross head area minus bar area

[2] Head dimensions updated from those given by Shao et al. (2016) (Ghimire et al. 2018)

[3] Octagonal head 
Table A.1 Cont. Details of cold-swaged threaded coupling sleeve headed bars

\begin{tabular}{|c|c|c|c|c|c|c|c|c|}
\hline & Dimension Notation & Designation & $\begin{array}{l}\text { Bar } \\
\text { Size }\end{array}$ & $\begin{array}{c}d_{1} \\
\text { (in.) }\end{array}$ & $\begin{array}{c}t_{1} \\
\text { (in.) }\end{array}$ & $\begin{array}{c}d_{2} \\
\text { (in.) }\end{array}$ & $\begin{array}{c}t_{2} \\
\text { (in.) }\end{array}$ & $\begin{array}{c}\text { Net } \\
\text { Bearing } \\
\text { Area }\left(A_{b r g}\right)\end{array}$ \\
\hline \multirow{8}{*}{ 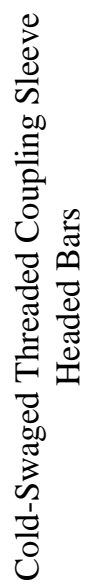 } & \multirow{8}{*}{$d_{1} \circlearrowleft \frac{d_{2}}{x}$} & \multirow{2}{*}{$\mathrm{O} 4.5^{[1]}$} & \multirow{2}{*}{ No. 8} & \multirow{2}{*}{2.76} & \multirow{2}{*}{1.625} & $1.72^{[2]}$ & \multirow{2}{*}{5.19} & $4.6 A_{b}{ }^{[4]}$ \\
\hline & & & & & & $2.2^{[3]}$ & & $2.8 A_{b}{ }^{[5]}$ \\
\hline & & \multirow{2}{*}{$\mathrm{O} 4.5^{[1]}$} & \multirow{2}{*}{ No. 11} & \multirow{2}{*}{3.75} & \multirow{2}{*}{2.17} & $2.28^{[2]}$ & \multirow{2}{*}{6.69} & $4.5 A_{b}{ }^{[4]}$ \\
\hline & & & & & & $2.86^{[3]}$ & & $3.0 A_{b}{ }^{[5]}$ \\
\hline & & \multirow{2}{*}{ O9.1 ${ }^{[1]}$} & \multirow{2}{*}{ No. 8} & \multirow{2}{*}{3.5} & \multirow{2}{*}{1.625} & $1.72^{[2]}$ & \multirow{2}{*}{5.19} & $9.2 A_{b}{ }^{[4]}$ \\
\hline & & & & & & $2.2^{[3]}$ & & $7.4 A_{b}[5]$ \\
\hline & & \multirow{2}{*}{ O12.9 $9^{[1]}$} & \multirow{2}{*}{ No. 8} & \multirow{2}{*}{4} & \multirow{2}{*}{1.625} & $1.72^{[2]}$ & \multirow{2}{*}{5.19} & $13.0 A_{b}^{[4]}$ \\
\hline & & & & & & $2.2^{[3]}$ & & $11.2 A_{b}{ }^{[5]}$ \\
\hline
\end{tabular}

${ }^{[1]}$ Head dimensions updated from those given by Shao et al. (2016). These heads contained obstructions with a gap (width not less than $3 / 8$ in.) adjacent to the bearing face of the head, as shown in Fig. A2 (Ghimire et al. 2018).

[2] Based on size of obstruction adjacent to head

[3] Based on maximum size of obstruction

[4] Net bearing area calculated as gross head area minus area of the obstruction adjacent to the bearing face of the head

[5] Net bearing area calculated as gross head area minus the maximum area of the obstruction

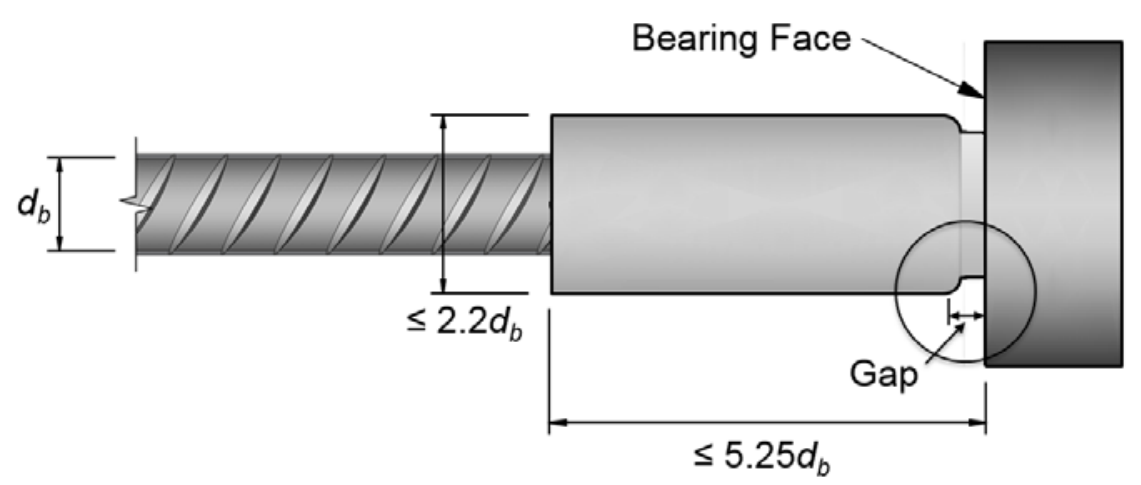

Fig. A2 Maximum dimensions and non-planner features of the obstruction 


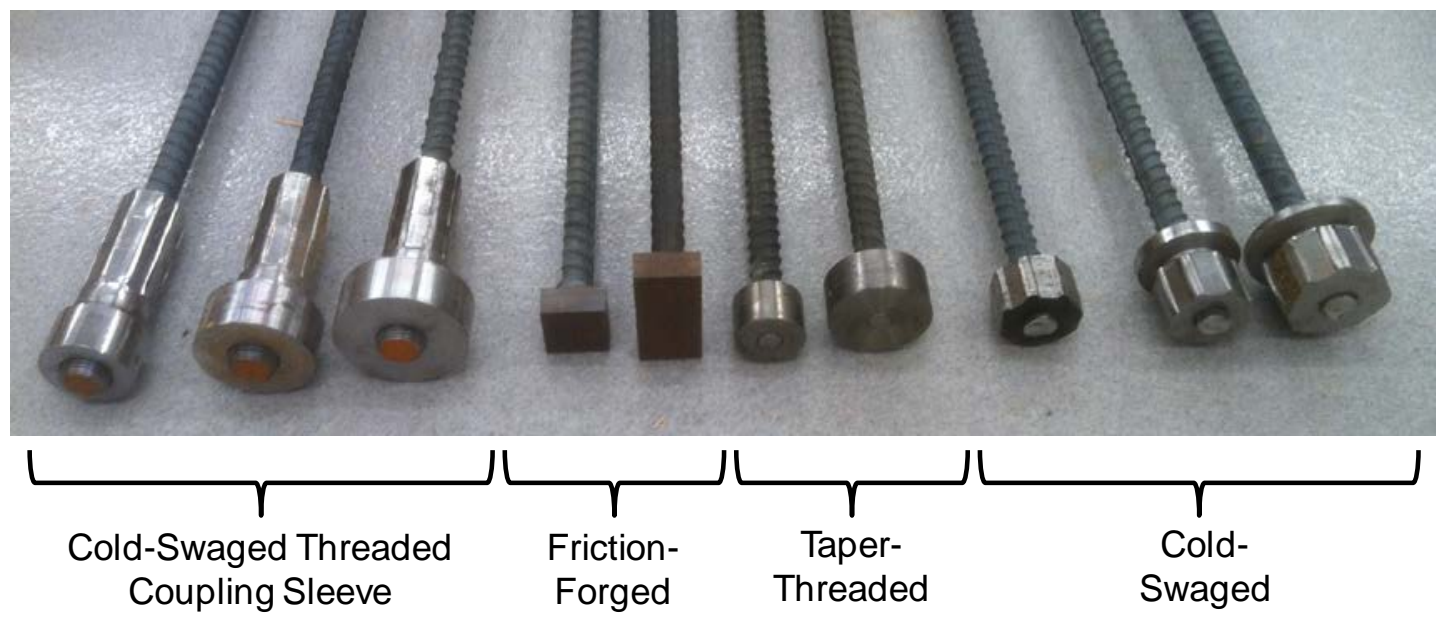

(a)

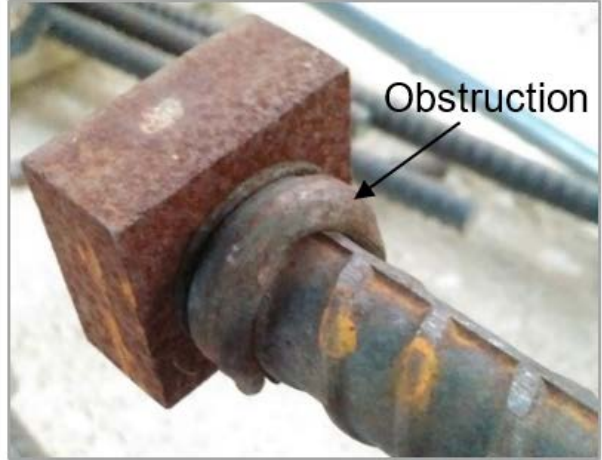

(b)

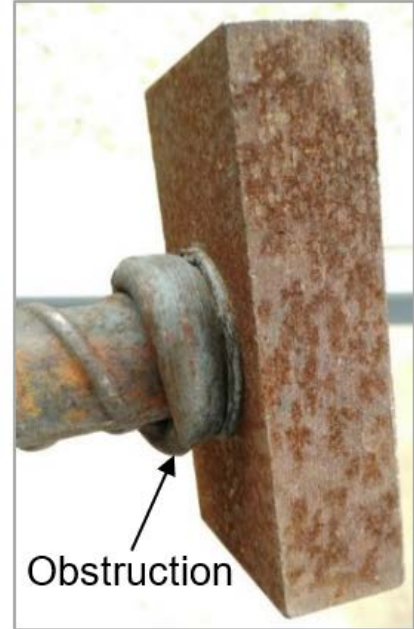

(c)

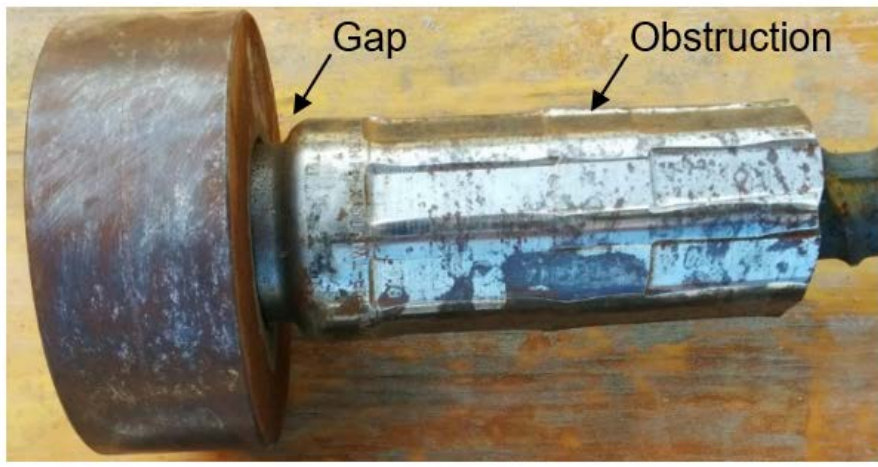

(d)

Fig. A3 (a) Left to right: cold-swaged threaded coupling sleeve, friction-forged, taper-threaded, and cold-swaged headed bars; (b) and (c) obstruction adjacent to the bearing face of the frictionforged heads; and (d) gap in the obstruction adjacent to the bearing face of the cold-swaged threaded coupling sleeve heads (taper-threaded and cold-swaged heads had no obstructions) 


\section{A.3 LONGITUDINAL COLUMN STEEL LAYOUTS}

The longitudinal column reinforcement layouts A.1 through A.17 shown below may not reflect the real size, number, and location of headed bars. Confining reinforcement is omitted in the layouts for clarity.

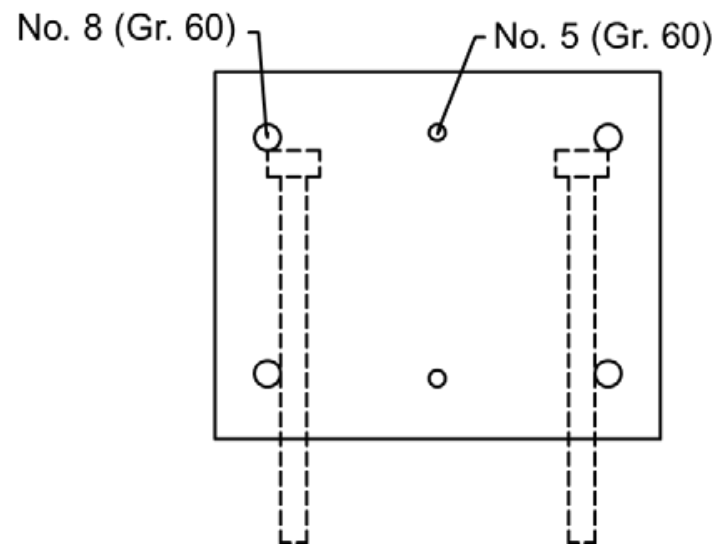

Layout A1 Layout B1: 4 No. 8 + 2 No. 5 (Gr. 60)

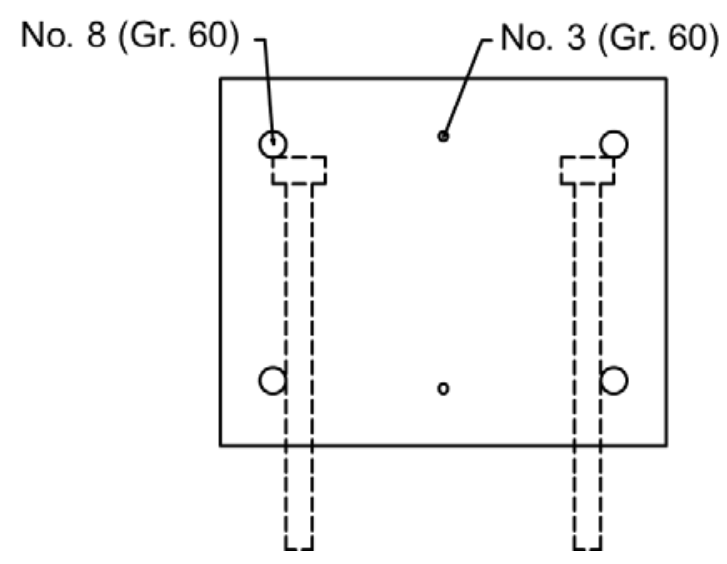

Layout A2 Layout B2: 4 No. 8 + 2 No. 3 (Gr. 60) 
No. 8 (Gr. 60)

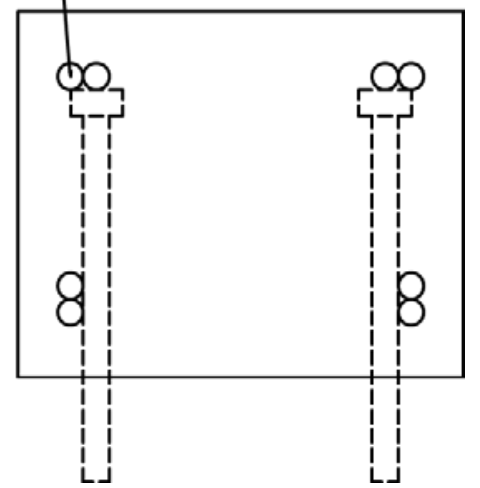

Layout A3 Layout B3: 8 No. 8 (Gr. 60), bundled at corner

No. 8 (Gr. 60)

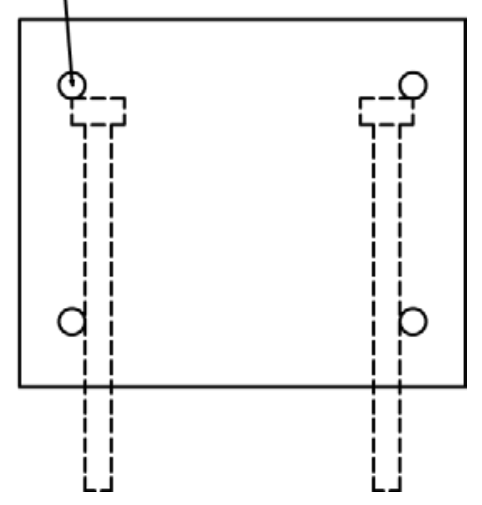

Layout A4 Layout B4: 4 No. 8 (Gr. 60)

No. 8 (Gr. 120)

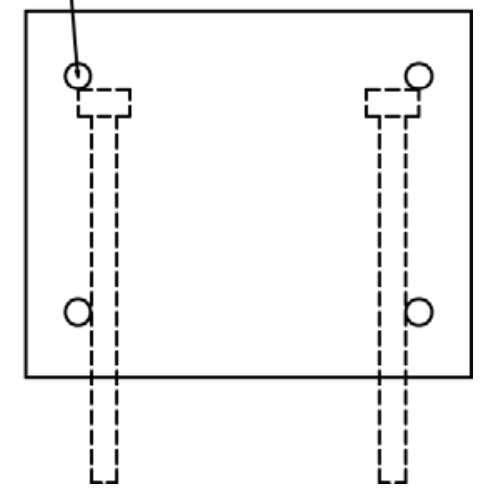

Layout A5 Layout B5: 4 No. 8 (Gr. 120) 
No. 8 (Gr. 60)

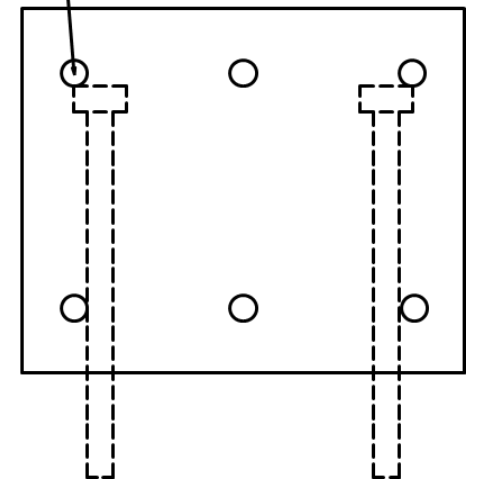

Layout A6 Layout B6: 6 No. 8 (Gr. 60)

No. 8 (Gr. 60)

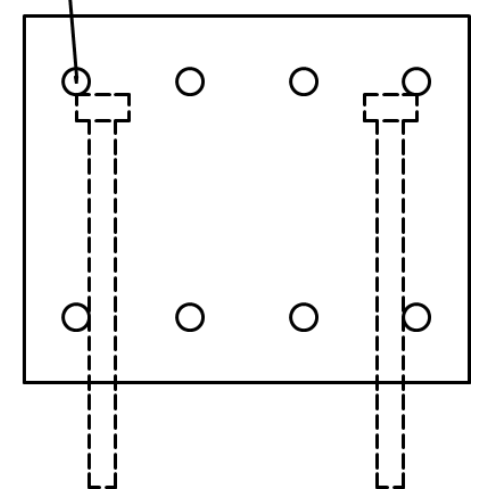

Layout A7 Layout B7: 8 No. 8 (Gr. 60)

No. 8 (Gr. 60)

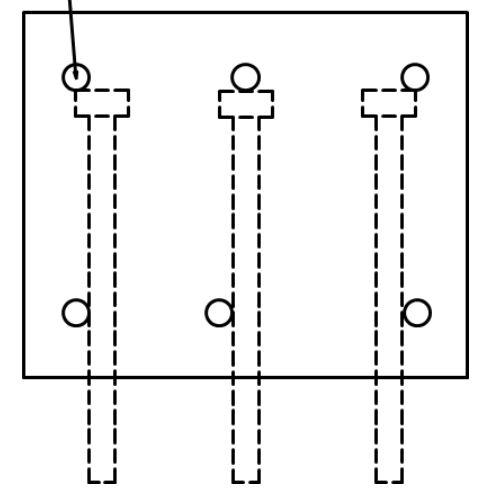

Layout A8 Layout B8: 6 No. 8 (Gr. 60), non-symmetric 
No. 8 (Gr. 80)

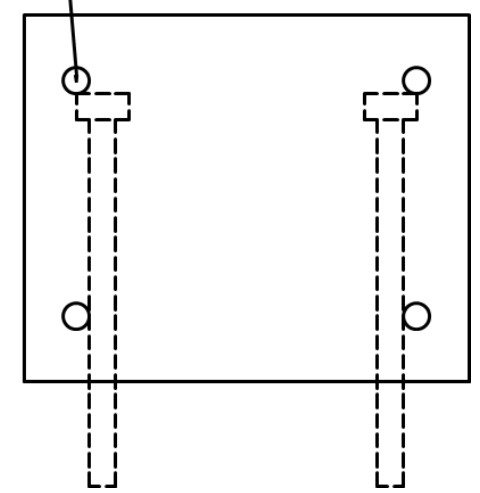

Layout A9 Layout B9: 4 No. 8 (Gr. 80)

No. 8 (Gr. 120)

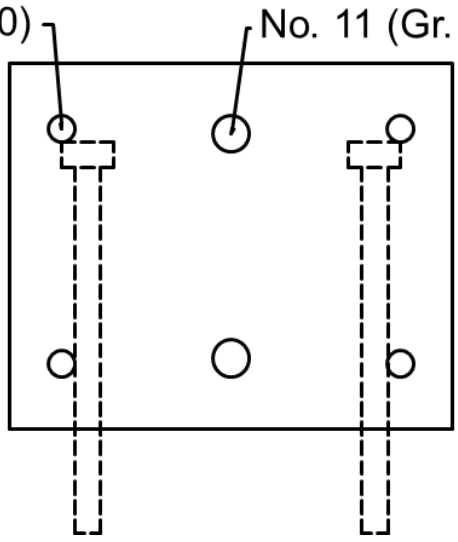

Layout A10 Layout B10: 4 No. 8 + 2 No. 11 (Gr. 120)

No. 8 (Gr. 120)

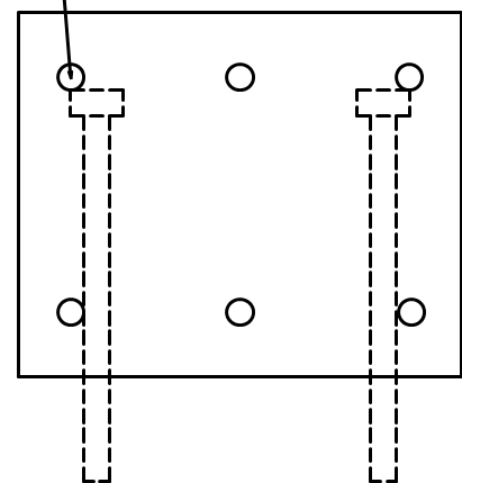

Layout A11 Layout B11: 6 No. 8 (Gr. 120) 


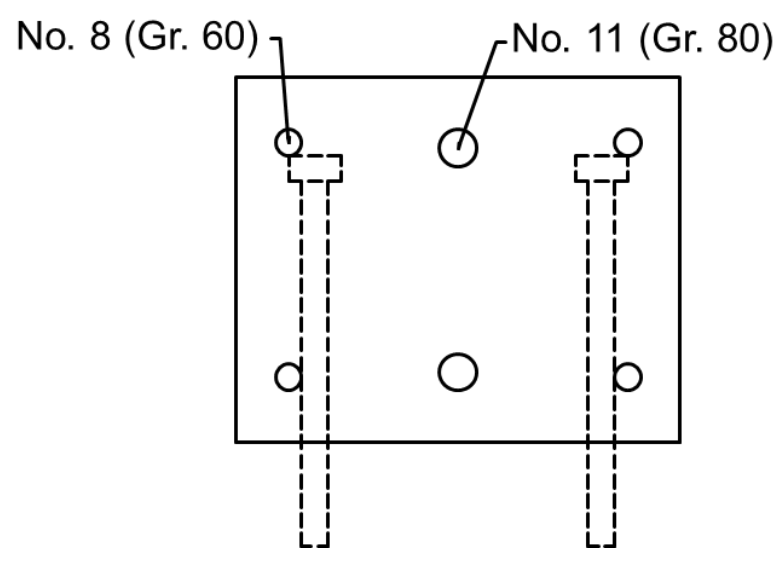

Layout A12 Layout B12: 4 No. 8 (Gr. 60) + 2 No. 11 (Gr. 80)

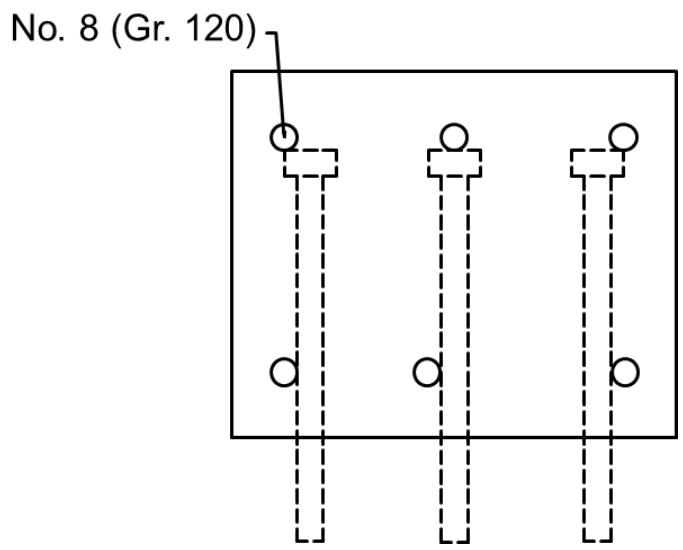

Layout A13 Layout B13: 6 No. 8 (Gr. 120), non-symmetric

No. 8 (Gr. 120)

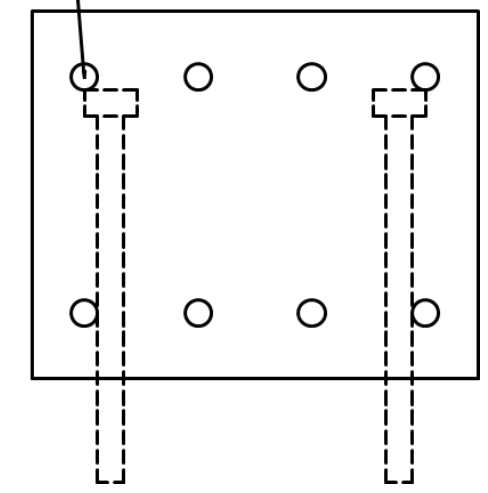

Layout A14 Layout B14: 8 No. 8 (Gr. 120) 


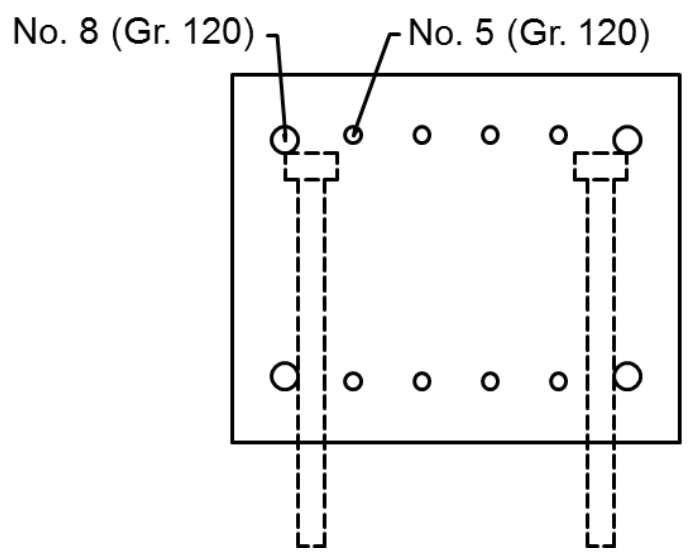

Layout A15 Layout B15: 4 No. 8 +8 No. 5 (Gr. 120)

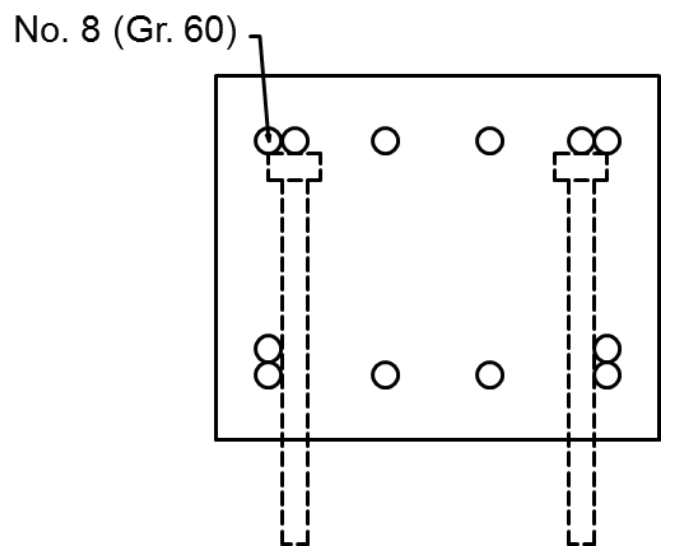

Layout A16 Layout B16: 12 No. 8 (Gr. 60)

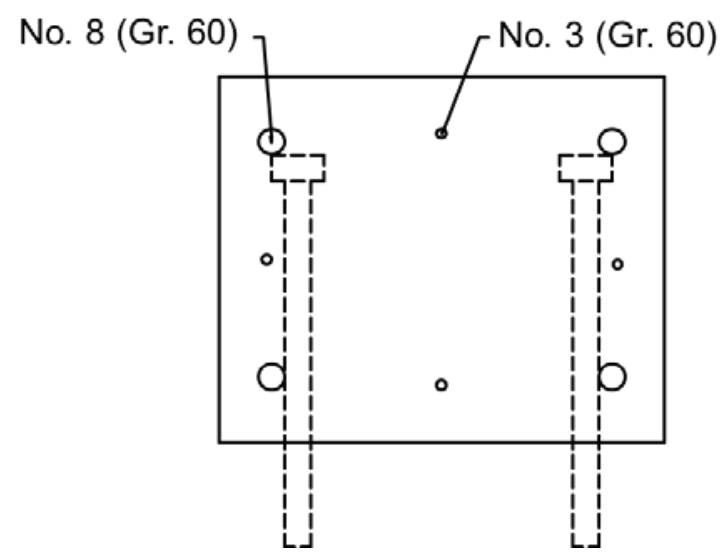

Layout A17 Layout B17: 4 No. 8 + 4 No. 3 (Gr. 60) 


\section{A.4 TEST-TO-CALCULATED RATIOS}

Table A.2 Test-to-calculated ratios for beam-column joint specimens with widely spaced headed bars and no confining reinforcement ${ }^{*}$

\begin{tabular}{|c|c|c|c|c|}
\hline & \multirow[b]{2}{*}{ Specimen } & \multirow[b]{2}{*}{$\begin{array}{c}T \\
\text { kips }\end{array}$} & \multicolumn{2}{|c|}{ Descriptive Equation $^{a}$} \\
\hline & & & $\begin{array}{c}T_{c} \\
\text { kips }\end{array}$ & $T / T_{c}$ \\
\hline 1 & 8-5g-T4.0-0-i-2.5-3-12.5 & 97.7 & 85.1 & 1.15 \\
\hline 2 & 8-5g-T4.0-0-i-3.5-3-12.5 & 93.4 & 86.0 & 1.09 \\
\hline 3 & 8-5-T4.0-0-i-2.5-3-12.5 & 83.3 & 86.3 & 0.96 \\
\hline 4 & 8-5-T4.0-0-i-3.5-3-12.5 & 91.9 & 87.5 & 1.05 \\
\hline 5 & 8-8-F4.1-0-i-2.5-3-10.5 & 77.1 & 77.1 & 1.00 \\
\hline 6 & 8-12-F4.1-0-i-2.5-3-10 & 71.8 & 76.8 & 0.93 \\
\hline 7 & 8-5-S6.5-0-i-2.5-3-11.25 & 75.6 & 73.4 & 1.03 \\
\hline 8 & 8-5-S6.5-0-i-2.5-3-14.25 & 87.7 & 95.2 & 0.92 \\
\hline 9 & $8-5-04.5-0-\mathrm{i}-2.5-3-11.25$ & 67.4 & 74.6 & 0.90 \\
\hline 10 & 8-5-O4.5-0-i-2.5-3-14.25 & 85.0 & 94.4 & 0.90 \\
\hline 11 & 8-5-T9.5-0-i-2.5-3-14.5 & 91.7 & 93.8 & 0.98 \\
\hline 12 & 8-5-09.1-0-i-2.5-3-14.5 & 94.8 & 93.8 & 1.01 \\
\hline 13 & 8-15-T4.0-0-i-2.5-4.5-9.5 & 83.3 & 81.1 & 1.03 \\
\hline 14 & 8-15-S9.5-0-i-2.5-3-9.5 & 81.7 & 81.1 & 1.01 \\
\hline 15 & 8-8-T9.5-0-i-2.5-3-9.5 & 65.2 & 69.7 & 0.94 \\
\hline 16 & (2@9)8-12-F4.1-0-i-2.5-3-12 & 79.1 & 96.9 & 0.82 \\
\hline 17 & (2@9)8-12-F9.1-0-i-2.5-3-12 & 76.5 & 95.3 & 0.80 \\
\hline 18 & 8-8-04.5-0-i-2.5-3-9.5 & 58.4 & 63.6 & 0.92 \\
\hline 19 & (2@9)8-8-O4.5-0-i-2.5-3-9.5 & 58.8 & 62.2 & 0.94 \\
\hline 20 & (2@9)8-8-T4.0-0-i-2.5-3-9.5 & 61.8 & 65.1 & 0.95 \\
\hline 21 & 5-5-F4.0-0-i-2.5-5-4 & 24.5 & 21.5 & 1.14 \\
\hline 22 & 5-5-F4.0-0-i-2.5-3-6 & 32.7 & 31.9 & 1.03 \\
\hline 23 & 5-12-F4.0-0-i-2.5-5-4 & 28.3 & 26.2 & 1.08 \\
\hline 24 & 5-12-F4.0-0-i-2.5-3-6 & 41.7 & 39.2 & 1.06 \\
\hline 25 & 11-5a-F3.8-0-i-2.5-3-17 & 97.5 & 116.5 & 0.84 \\
\hline 26 & 11-5-F3.8-0-i-2.5-3-17 & 132.7 & 132.2 & 1.00 \\
\hline 27 & 11-12-O4.5-0-i-2.5-3-16.75 & 169.6 & 152.8 & 1.11 \\
\hline 28 & 11-12-S5.5-0-i-2.5-3-16.75 & 175.9 & 148.5 & 1.18 \\
\hline 29 & 11-5-O4.5-0-i-2.5-3-19.25 & 157.9 & 147.4 & 1.07 \\
\hline 30 & 11-5-S5.5-0-i-2.5-3-19.25 & 176.8 & 152.4 & 1.16 \\
\hline
\end{tabular}

* Specimens used to develop descriptive equations

${ }^{\text {a }} T_{C}$ based on Eq. (3) 
Table A.2 Cont. Test-to-calculated ratios for beam-column joint specimens with closely spaced headed bars and no confining reinforcement ${ }^{*}$

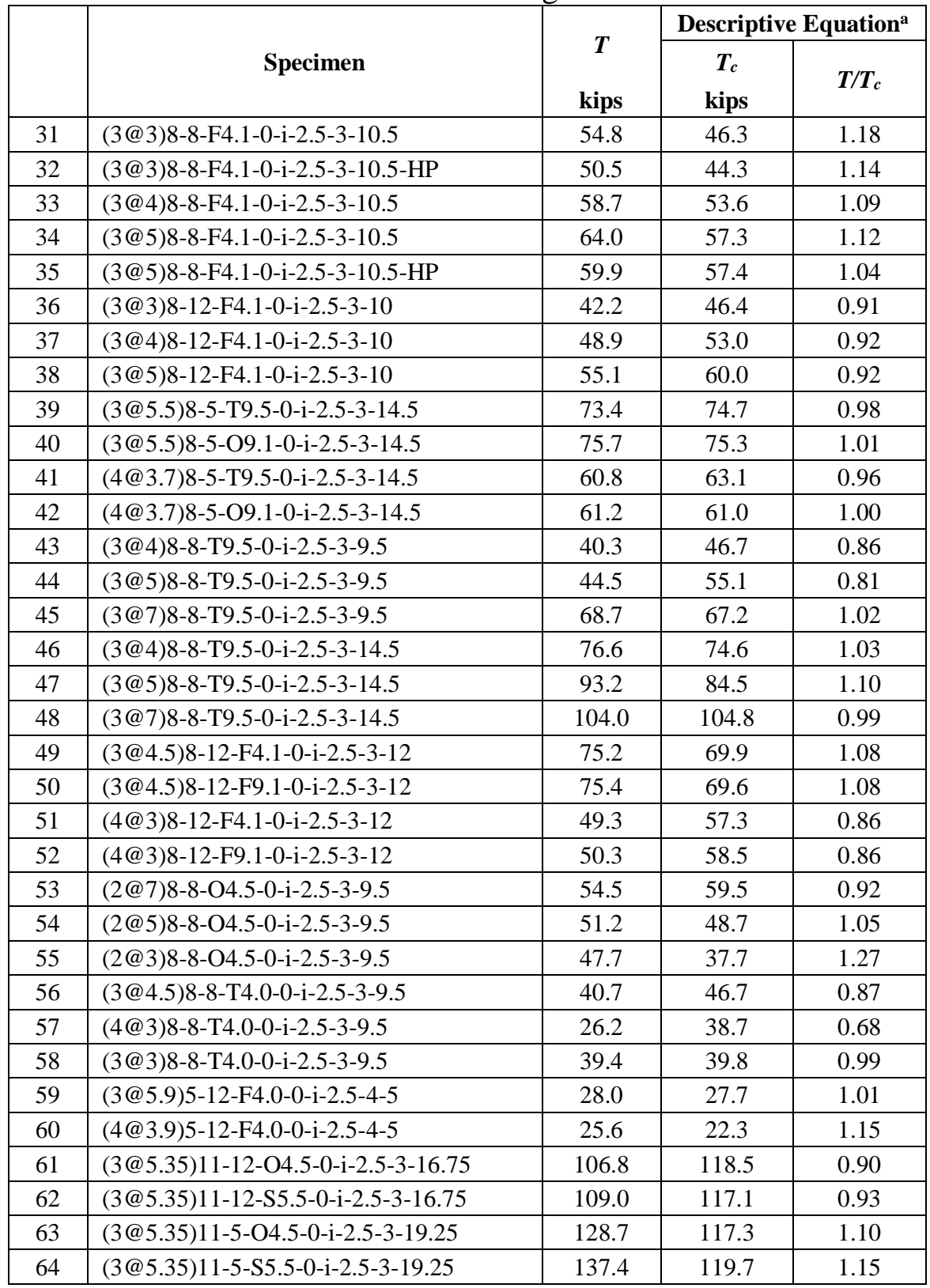

${ }^{*}$ Specimens used to develop descriptive equations

${ }^{\mathrm{a}} T_{c}$ based on Eq. (4) 
Table A.2 Cont. Test-to-calculated ratios for beam-column joint specimens with widely spaced headed bars with confining reinforcement ${ }^{*}$

\begin{tabular}{|c|c|c|c|c|}
\hline & \\
\hline & \multirow[b]{2}{*}{ Specimen } & \multirow[b]{2}{*}{$\begin{array}{c}T \\
\text { kips }\end{array}$} & \multicolumn{2}{|c|}{ Descriptive Equation $^{\mathrm{a}}$} \\
\hline & & & $\begin{array}{c}T_{h} \\
\text { kips }\end{array}$ & $T / T_{h}$ \\
\hline 65 & 8-5-T4.0-4\#3-i-3-3-12.5 & 87.5 & 96.9 & 0.90 \\
\hline 66 & 8-5-T4.0-4\#3-i-4-3-12.5 & 96.2 & 95.9 & 1.00 \\
\hline 67 & 8-5-T4.0-4\#4-i-3-3-12.5 & 109.0 & 100.7 & 1.08 \\
\hline 68 & 8-5-T4.0-4\#4-i-4-3-12.5 & 101.5 & 98.2 & 1.03 \\
\hline 69 & 8-5g-T4.0-5\#3-i-2.5-3-9.5 & 78.7 & 78.1 & 1.01 \\
\hline 70 & 8-5g-T4.0-5\#3-i-3.5-3-9.5 & 79.5 & 80.3 & 0.99 \\
\hline 71 & 8-5g-T4.0-4\#4-i-2.5-3-9.5 & 90.7 & 79.2 & 1.14 \\
\hline 72 & 8-5g-T4.0-4\#4-i-3.5-3-9.5 & 96.7 & 83.3 & 1.16 \\
\hline 73 & 8-5-T4.0-5\#3-i-2.5-3-9.5 & 74.2 & 78.7 & 0.94 \\
\hline 74 & 8-5-T4.0-5\#3-i-3.5-3-9.5 & 80.6 & 78.2 & 1.03 \\
\hline 75 & 8-5-T4.0-4\#4-i-2.5-3-9.5 & 90.5 & 82.9 & 1.09 \\
\hline 76 & 8-5-T4.0-4\#4-i-3.5-3-9.5 & 85.6 & 82.3 & 1.04 \\
\hline 77 & 8-8-F4.1-2\#3-i-2.5-3-10 & 73.4 & 77.7 & 0.94 \\
\hline 78 & 8-12-F4.1-5\#3-i-2.5-3-10 & 87.2 & 95.5 & 0.91 \\
\hline 79 & 8-5-S6.5-2\#3-i-2.5-3-9.25 & 63.4 & 66.2 & 0.96 \\
\hline 80 & 8-5-S6.5-2\#3-i-2.5-3-12.25 & 86.0 & 88.2 & 0.97 \\
\hline 81 & 8-5-O4.5-2\#3-i-2.5-3-9.25 & 67.9 & 67.9 & 1.00 \\
\hline 82 & 8-5-O4.5-2\#3-i-2.5-3-12.25 & 78.5 & 86.0 & 0.91 \\
\hline 83 & 8-5-S6.5-5\#3-i-2.5-3-8.25 & 62.0 & 71.7 & 0.87 \\
\hline 84 & 8-5-S6.5-5\#3-i-2.5-3-11.25 & 84.5 & 89.9 & 0.94 \\
\hline 85 & 8-5-O4.5-5\#3-i-2.5-3-8.25 & 68.4 & 69.5 & 0.98 \\
\hline 86 & 8-5-O4.5-5\#3-i-2.5-3-11.25 & 82.2 & 91.2 & 0.90 \\
\hline 87 & 8-5-T9.5-5\#3-i-2.5-3-14.5 & 121.0 & 111.9 & 1.08 \\
\hline 88 & 8-15-T4.0-2\#3-i-2.5-4.5-7 & 59.0 & 65.1 & 0.91 \\
\hline 89 & 8-15-S9.5-2\#3-i-2.5-3-7 & 67.1 & 65.1 & 1.03 \\
\hline 90 & 8-15-T4.0-5\#3-i-2.5-4.5-5.5 & 63.3 & 62.3 & 1.02 \\
\hline 91 & 8-15-S9.5-5\#3-i-2.5-3-5.5 & 75.8 & 63.4 & 1.20 \\
\hline 92 & 8-8-T9.5-2\#3-i-2.5-3-9.5 & 68.7 & 73.6 & 0.93 \\
\hline 93 & (2@9)8-12-F4.1-5\#3-i-2.5-3-12 & 111.9 & 112.2 & 1.00 \\
\hline 94 & (2@9)8-8-T4.0-5\#3-i-2.5-3-9.5 & 76.7 & 82.1 & 0.93 \\
\hline 95 & 5-5-F4.0-2\#3-i-2.5-5-4 & 19.7 & 23.7 & 0.83 \\
\hline 96 & 5-5-F4.0-5\#3-i-2.5-5-4 & 26.5 & 32.6 & 0.81 \\
\hline 97 & 5-5-F4.0-2\#3-i-2.5-3-6 & 37.9 & 35.4 & 1.07 \\
\hline 98 & 5-5-F4.0-5\#3-i-2.5-3-6 & 43.5 & 42.9 & 1.01 \\
\hline 99 & 5-12-F4.0-2\#3-i-2.5-5-4 & 32.7 & 30.2 & 1.08 \\
\hline 100 & 5-12-F4.0-5\#3-i-2.5-5-4 & 38.9 & 37.9 & 1.03 \\
\hline 101 & 11-5a-F3.8-2\#3-i-2.5-3-17 & 118.2 & 130.1 & 0.91 \\
\hline 102 & 11-5a-F3.8-6\#3-i-2.5-3-17 & 116.2 & 139.4 & 0.83 \\
\hline 103 & 11-5-F3.8-6\#3-i-2.5-3-17 & 151.9 & 152.7 & 1.00 \\
\hline 104 & 11-12-04.5-6\#3-i-2.5-3-16.75 & 201.5 & 171.7 & 1.17 \\
\hline 105 & 11-12-S5.5-6\#3-i-2.5-3-16.75 & 197.4 & 169.2 & 1.17 \\
\hline 106 & 11-5-O4.5-6\#3-i-2.5-3-19.25 & 181.4 & 170.7 & 1.06 \\
\hline 107 & 11-5-S5.5-6\#3-i-2.5-3-19.25 & 189.6 & 172.1 & 1.10 \\
\hline
\end{tabular}

${ }^{*}$ Specimens used to develop descriptive equations

${ }^{\text {a }} T_{h}$ based on Eq. (6) 
Table A.2 Cont. Test-to-calculated ratios for beam-column joint specimens with closely spaced headed bars with confining reinforcement ${ }^{*}$

\begin{tabular}{|c|c|c|c|c|}
\hline & \multirow[b]{2}{*}{ Specimen } & \multirow[b]{2}{*}{$\begin{array}{c}T \\
\text { kips }\end{array}$} & \multicolumn{2}{|c|}{ Descriptive Equation $^{\mathbf{a}}$} \\
\hline & & & $\begin{array}{c}T_{h} \\
\text { kips }\end{array}$ & $T / T_{h}$ \\
\hline 108 & (3@3)8-8-F4.1-2\#3-i-2.5-3-10 & 61.9 & 55.9 & 1.11 \\
\hline 109 & (3@3)8-8-F4.1-2\#3-i-2.5-3-10-HP & 56.7 & 57.4 & 0.99 \\
\hline 110 & (3@4)8-8-F4.1-2\#3-i-2.5-3-10 & 55.5 & 59.4 & 0.93 \\
\hline 111 & (3@4)8-8-F4.1-2\#3-i-2.5-3-10-HP & 69.8 & 62.8 & 1.11 \\
\hline 112 & (3@5)8-8-F4.1-2\#3-i-2.5-3-10.5 & 56.1 & 62.8 & 0.89 \\
\hline 113 & (3@5)8-8-F4.1-2\#3-i-2.5-3-10.5-HP & 65.5 & 65.6 & 1.00 \\
\hline 114 & (3@3)8-12-F4.1-5\#3-i-2.5-3-10 & 61.6 & 65.2 & 0.95 \\
\hline 115 & (3@4)8-12-F4.1-5\#3-i-2.5-3-10 & 65.7 & 69.3 & 0.95 \\
\hline 116 & (3@5)8-12-F4.1-5\#3-i-2.5-3-10 & 69.7 & 74.6 & 0.93 \\
\hline 117 & (3@5.5)8-5-T9.5-5\#3-i-2.5-3-14.5 & 94.6 & 94.7 & 1.00 \\
\hline 118 & (4@3.7)8-5-T9.5-5\#3-i-2.5-3-14.5 & 76.9 & 81.7 & 0.94 \\
\hline 119 & (3@4)8-8-T9.5-2\#3-i-2.5-3-9.5 & 51.8 & 59.3 & 0.87 \\
\hline 120 & (3@5)8-8-T9.5-2\#3-i-2.5-3-9.5 & 55.9 & 64.2 & 0.87 \\
\hline 121 & (3@7)8-8-T9.5-2\#3-i-2.5-3-9.5 & 67.6 & 75.6 & 0.89 \\
\hline 122 & (3@4)8-8-T9.5-2\#3-i-2.5-3-14.5 & 85.4 & 88.8 & 0.96 \\
\hline 123 & (3@5)8-8-T9.5-2\#3-i-2.5-3-14.5 & 105.2 & 95.6 & 1.10 \\
\hline 124 & (3@7)8-8-T9.5-2\#3-i-2.5-3-14.5 & 113.4 & 113.8 & 1.00 \\
\hline 125 & (3@4.5)8-12-F4.1-5\#3-i-2.5-3-12 & 87.7 & 89.0 & 0.99 \\
\hline 126 & (3@4.5)8-12-F9.1-5\#3-i-2.5-3-12 & 108.6 & 87.3 & 1.24 \\
\hline 127 & (4@3)8-12-F4.1-5\#3-i-2.5-3-12 & 64.2 & 77.0 & 0.83 \\
\hline 128 & (4@3)8-12-F9.1-5\#3-i-2.5-3-12 & 87.8 & 76.3 & 1.15 \\
\hline 129 & (3@4.5)8-8-T4.0-5\#3-i-2.5-3-9.5 & 62.5 & 61.7 & 1.01 \\
\hline 130 & (4@3)8-8-T4.0-5\#3-i-2.5-3-9.5 & 48.6 & 54.8 & 0.89 \\
\hline 131 & (3@3)8-8-T4.0-5\#3-i-2.5-3-9.5 & 56.5 & 55.1 & 1.03 \\
\hline 132 & (3@5.9)5-12-F4.0-2\#3-i-2.5-4-5 & 35.1 & 32.8 & 1.07 \\
\hline 133 & (3@5.9)5-12-F4.0-5\#3-i-2.5-4-5 & 38.6 & 36.1 & 1.07 \\
\hline 134 & (4@3.9)5-12-F4.0-2\#3-i-2.5-4-5 & 30.9 & 27.1 & 1.14 \\
\hline 135 & (3@5.35)11-12-04.5-6\#3-i-2.5-3-16.75 & 135.8 & 145.6 & 0.93 \\
\hline 136 & (3@5.35)11-12-S5.5-6\#3-i-2.5-3-16.75 & 153.8 & 140.5 & 1.09 \\
\hline 137 & (3@5.35)11-5-O4.5-6\#3-i-2.5-3-19.25 & 141.7 & 141.9 & 1.00 \\
\hline 138 & (3@5.35)11-5-S5.5-6\#3-i-2.5-3-19.25 & 152.9 & 144.9 & 1.06 \\
\hline
\end{tabular}

* Specimens used to develop descriptive equations

${ }^{\text {a }} T_{h}$ based on Eq. (7) 


\section{A.5 COMPREHENSIVE TEST RESULTS}

Table A.3 Comprehensive test results and data for beam-column joint specimens

\begin{tabular}{|c|c|c|c|c|c|c|c|c|c|c|}
\hline & Specimen & Head & $\begin{array}{l}\boldsymbol{c}_{\boldsymbol{o}} \\
\text { in. }\end{array}$ & $A_{b r g}$ & $\begin{array}{l}\ell \text { eh } \\
\text { in. }\end{array}$ & $\begin{array}{c}\ell \text { eh, avg } \\
\text { in. }\end{array}$ & $\begin{array}{l}\boldsymbol{f}_{\boldsymbol{c m}} \\
\mathrm{psi}\end{array}$ & $\begin{array}{l}\text { Age } \\
\text { days }\end{array}$ & $\begin{array}{l}\boldsymbol{d}_{\boldsymbol{b}} \\
\text { in. }\end{array}$ & $\begin{array}{l}\boldsymbol{A}_{\boldsymbol{b}} \\
\text { in. }^{2}\end{array}$ \\
\hline \multirow{6}{*}{ Group 1} & $8-5-T 4.0-0-i-3-3-15.5^{\mathrm{a}}$ & $\begin{array}{l}\text { A } \\
\text { B }\end{array}$ & 2.4 & $4.0 A_{b}$ & $\begin{array}{l}15.50 \\
16.00\end{array}$ & 15.75 & 4850 & 7 & 1 & 0.79 \\
\hline & $8-5-T 4.0-0-i-4-3-15.5^{a b}$ & $\begin{array}{l}\text { A } \\
B\end{array}$ & 3.4 & $4.0 A_{b}$ & $\begin{array}{l}15.50 \\
15.06\end{array}$ & 15.28 & 5070 & 8 & 1 & 0.79 \\
\hline & $8-5-T 4.0-4 \# 3-i-3-3-12.5^{a}$ & $\begin{array}{l}\text { A } \\
B\end{array}$ & 2.4 & $4.0 A_{b}$ & $\begin{array}{l}12.06 \\
12.69\end{array}$ & 12.38 & 5070 & 8 & 1 & 0.79 \\
\hline & $8-5-T 4.0-4 \# 3-\mathrm{i}-4-3-12.5^{\mathrm{a}}$ & $\begin{array}{l}\mathrm{A} \\
\mathrm{B}\end{array}$ & 3.4 & $4.0 A_{b}$ & $\begin{array}{l}11.94 \\
12.19 \\
\end{array}$ & 12.06 & 5380 & 11 & 1 & 0.79 \\
\hline & $8-5-T 4.0-4 \# 4-i-3-3-12.5^{a}$ & $\begin{array}{l}\mathrm{A} \\
\mathrm{B}\end{array}$ & 2.4 & $4.0 A_{b}$ & $\begin{array}{l}12.56 \\
12.31 \\
\end{array}$ & 12.44 & 5070 & 8 & 1 & 0.79 \\
\hline & $8-5-T 4.0-4 \# 4-\mathrm{i}-4-3-12.5^{\mathrm{a}}$ & $\begin{array}{l}\mathrm{A} \\
\mathrm{B} \\
\end{array}$ & 3.4 & $4.0 A_{b}$ & $\begin{array}{l}12.06 \\
12.31 \\
\end{array}$ & 12.19 & 4850 & 7 & 1 & 0.79 \\
\hline \multirow{6}{*}{ Group 2} & $8-5 g-T 4.0-0-i-2.5-3-12.5^{a}$ & $\begin{array}{l}\text { A } \\
B\end{array}$ & 1.9 & $4.0 A_{b}$ & $\begin{array}{l}12.69 \\
12.44\end{array}$ & 12.56 & 5910 & 14 & 1 & 0.79 \\
\hline & $8-5 g-T 4.0-0-i-3.5-3-12.5^{a}$ & $\begin{array}{l}\mathrm{A} \\
\mathrm{B} \\
\end{array}$ & 2.9 & $4.0 A_{b}$ & $\begin{array}{l}12.44 \\
12.56 \\
\end{array}$ & 12.50 & 6320 & 15 & 1 & 0.79 \\
\hline & $8-5 g-T 4.0-5 \# 3-i-2.5-3-9.5^{a}$ & $\begin{array}{l}\mathrm{A} \\
\mathrm{B}\end{array}$ & 1.9 & $4.0 A_{b}$ & $\begin{array}{l}9.44 \\
9.69 \\
\end{array}$ & 9.56 & 5090 & 7 & 1 & 0.79 \\
\hline & $8-5 g-T 4.0-5 \# 3-i-3.5-3-9.5^{a}$ & $\begin{array}{l}A \\
B\end{array}$ & 2.9 & $4.0 A_{b}$ & $\begin{array}{l}9.69 \\
9.44\end{array}$ & 9.56 & 5910 & 14 & 1 & 0.79 \\
\hline & $8-5 g-T 4.0-4 \# 4-i-2.5-3-9.5^{a}$ & $\begin{array}{l}\text { A } \\
B\end{array}$ & 1.9 & $4.0 A_{b}$ & $\begin{array}{l}9.44 \\
8.94 \\
\end{array}$ & 9.19 & 5180 & 8 & 1 & 0.79 \\
\hline & $8-5 g-T 4.0-4 \# 4-i-3.5-3-9.5^{a}$ & $\begin{array}{l}\text { A } \\
\text { B }\end{array}$ & 2.9 & $4.0 A_{b}$ & $\begin{array}{l}9.31 \\
9.69 \\
\end{array}$ & 9.50 & 5910 & 14 & 1 & 0.79 \\
\hline \multirow{6}{*}{ Group 3} & $8-5-T 4.0-0-i-2.5-3-12.5^{a}$ & $\begin{array}{l}\text { A } \\
\mathrm{B}\end{array}$ & 1.9 & $4.0 A_{b}$ & $\begin{array}{l}12.69 \\
12.50 \\
\end{array}$ & 12.59 & 6210 & 8 & 1 & 0.79 \\
\hline & $8-5-T 4.0-0-i-3.5-3-12.5^{a}$ & $\begin{array}{l}\text { A } \\
\text { B }\end{array}$ & 2.9 & $4.0 A_{b}$ & $\begin{array}{l}12.81 \\
12.50\end{array}$ & 12.66 & 6440 & 9 & 1 & 0.79 \\
\hline & $8-5-T 4.0-5 \# 3-i-2.5-3-9.5^{a}$ & $\begin{array}{l}\text { A } \\
B\end{array}$ & 1.9 & $4.0 A_{b}$ & $\begin{array}{l}9.44 \\
9.19 \\
\end{array}$ & 9.31 & 5960 & 7 & 1 & 0.79 \\
\hline & $8-5-T 4.0-5 \# 3-i-3.5-3-9.5^{a}$ & $\begin{array}{l}\text { A } \\
B\end{array}$ & 2.9 & $4.0 A_{b}$ & $\begin{array}{l}9.06 \\
9.06 \\
\end{array}$ & 9.06 & 6440 & 9 & 1 & 0.79 \\
\hline & $8-5-T 4.0-4 \# 4-i-2.5-3-9.5^{a}$ & $\begin{array}{l}\text { A } \\
\text { B }\end{array}$ & 1.9 & $4.0 A_{b}$ & $\begin{array}{l}9.19 \\
9.31 \\
\end{array}$ & 9.25 & 6440 & 9 & 1 & 0.79 \\
\hline & $8-5-T 4.0-4 \# 4-i-3.5-3-9.5^{a}$ & $\begin{array}{l}\text { A } \\
\text { B }\end{array}$ & 2.9 & $4.0 A_{b}$ & $\begin{array}{l}9.56 \\
8.94 \\
\end{array}$ & 9.25 & 6210 & 8 & 1 & 0.79 \\
\hline \multirow{3}{*}{ Group 4} & 8-8-F4.1-0-i-2.5-3-10.5 & $\begin{array}{l}\mathrm{A} \\
\mathrm{B}\end{array}$ & 2.0 & $4.1 A_{b}$ & $\begin{array}{l}10.25 \\
10.75\end{array}$ & 10.50 & 8450 & 9 & 1 & 0.79 \\
\hline & 8-8-F4.1-2\#3-i-2.5-3-10 & $\begin{array}{l}\text { A } \\
B\end{array}$ & 2.0 & $4.1 A_{b}$ & $\begin{array}{c}9.75 \\
10.00 \\
\end{array}$ & 9.88 & 8450 & 9 & 1 & 0.79 \\
\hline & (3@3)8-8-F4.1-0-i-2.5-3-10.5 & $\begin{array}{l}\text { A } \\
\text { B } \\
\text { C }\end{array}$ & 2.0 & $4.1 A_{b}$ & $\begin{array}{l}10.63 \\
10.75 \\
10.38\end{array}$ & 10.58 & 8450 & 9 & 1 & 0.79 \\
\hline
\end{tabular}

${ }^{\text {a }}$ Specimen contained crossties within joint region

${ }^{\mathrm{b}}$ Specimen had no confining reinforcement above joint region 
Table A.3 Cont. Comprehensive test results and data for beam-column joint specimens

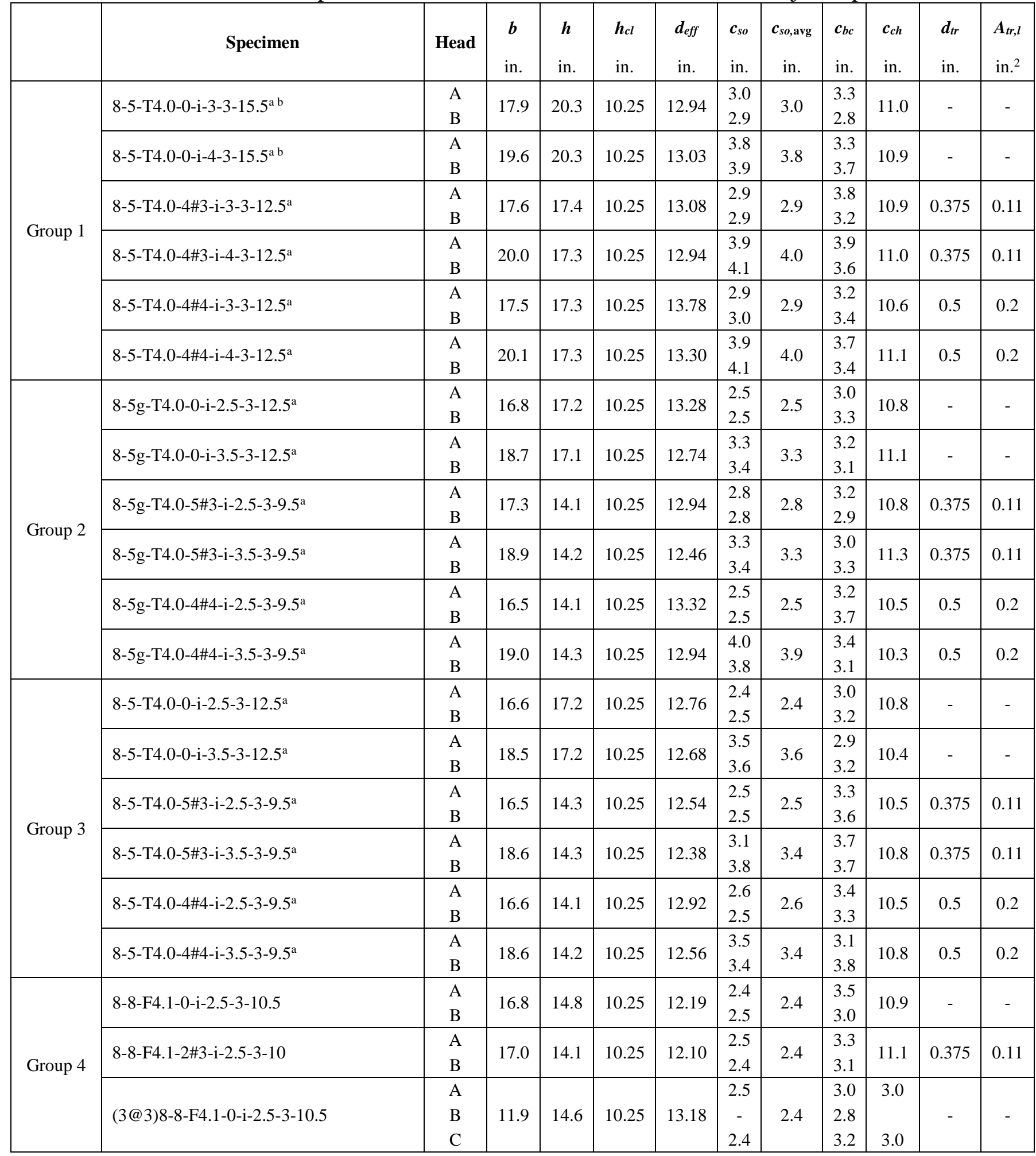

a Specimen contained crossties within joint region

b Specimen had no confining reinforcement above joint region 
Table A.3 Cont. Comprehensive test results and data for beam-column joint specimens

\begin{tabular}{|c|c|c|c|c|c|c|c|c|c|c|c|}
\hline & Specimen & Head & $N$ & $\begin{array}{l}s_{t r}{ }^{c} \\
\text { in. }\end{array}$ & $\begin{array}{l}\boldsymbol{A}_{t t} \\
\text { in. }^{2}\end{array}$ & $\begin{array}{l}\boldsymbol{d}_{\text {tro }} \\
\text { in. }\end{array}$ & $\begin{array}{l}S_{\text {tro }}^{\mathrm{C}} \\
\text { in. }\end{array}$ & $\begin{array}{l}A_{a b} \\
\text { in. }{ }^{2}\end{array}$ & $n$ & $\begin{array}{l}\boldsymbol{A}_{\boldsymbol{h s}} \\
\text { in. }^{2}\end{array}$ & $\begin{array}{l}\text { Long. } \\
\text { Reinf. } \\
\text { Layout }\end{array}$ \\
\hline \multirow{6}{*}{ Group 1} & $8-5-T 4.0-0-i-3-3-15.5^{a b}$ & $\begin{array}{l}\mathrm{A} \\
\mathrm{B}\end{array}$ & - & - & - & - & - & - & 2 & 1.58 & A1 \\
\hline & $8-5-T 4.0-0-i-4-3-15.5^{a}$ & $\begin{array}{l}\text { A } \\
B\end{array}$ & - & - & - & - & - & - & 2 & 1.58 & A1 \\
\hline & 8-5-T4.0-4\#3-i-3-3-12.5 & $\begin{array}{l}\text { A } \\
B\end{array}$ & 8 & $\begin{array}{c}3 \\
(1.5)\end{array}$ & 0.66 & 0.375 & $\begin{array}{c}3 \\
(1.5)\end{array}$ & 0.66 & 2 & 1.58 & A2 \\
\hline & $8-5-T 4.0-4 \# 3-\mathrm{i}-4-3-12.5^{\mathrm{a}}$ & $\begin{array}{l}\text { A } \\
B\end{array}$ & 8 & $\begin{array}{c}3 \\
(1.5) \\
\end{array}$ & 0.66 & 0.375 & $\begin{array}{c}3 \\
(1.5) \\
\end{array}$ & 0.66 & 2 & 1.58 & A2 \\
\hline & $8-5-T 4.0-4 \# 4-i-3-3-12.5^{a}$ & $\begin{array}{l}\mathrm{A} \\
\mathrm{B}\end{array}$ & 8 & $\begin{array}{c}4 \\
(2) \\
\end{array}$ & 0.80 & 0.5 & $\begin{array}{c}4 \\
(2) \\
\end{array}$ & 0.80 & 2 & 1.58 & A17 \\
\hline & $8-5-T 4.0-4 \# 4-\mathrm{i}-4-3-12.5^{\mathrm{a}}$ & $\begin{array}{l}\mathrm{A} \\
\mathrm{B}\end{array}$ & 8 & $\begin{array}{c}4 \\
(2) \\
\end{array}$ & 0.80 & 0.5 & $\begin{array}{c}4 \\
(2) \\
\end{array}$ & 0.80 & 2 & 1.58 & A17 \\
\hline \multirow{6}{*}{ Group 2} & $8-5 g-T 4.0-0-i-2.5-3-12.5^{a}$ & $\begin{array}{l}\text { A } \\
B\end{array}$ & - & - & - & 0.375 & $\begin{array}{c}3.5 \\
(1.75)\end{array}$ & 0.44 & 2 & 1.58 & A2 \\
\hline & $8-5 g-T 4.0-0-i-3.5-3-12.5^{a}$ & $\begin{array}{l}\text { A } \\
B\end{array}$ & - & - & - & 0.375 & $\begin{array}{c}3.5 \\
(1.75) \\
\end{array}$ & 0.44 & 2 & 1.58 & A2 \\
\hline & 8-5g-T4.0-5\#3-i-2.5-3-9.5 & $\begin{array}{l}A \\
B\end{array}$ & 10 & $\begin{array}{c}3 \\
(1.5)\end{array}$ & 0.66 & 0.375 & $\begin{array}{c}3.5 \\
(1.75)\end{array}$ & 0.44 & 2 & 1.58 & A2 \\
\hline & $8-5 g-T 4.0-5 \# 3-i-3.5-3-9.5^{a}$ & $\begin{array}{l}\text { A } \\
\text { B }\end{array}$ & 10 & $\begin{array}{c}3 \\
(1.5)\end{array}$ & 0.66 & 0.375 & $\begin{array}{c}3.5 \\
(1.75)\end{array}$ & 0.44 & 2 & 1.58 & $\mathrm{~A} 2$ \\
\hline & $8-5 g-T 4.0-4 \# 4-i-2.5-3-9.5^{a}$ & $\begin{array}{l}A \\
B\end{array}$ & 8 & $\begin{array}{c}4 \\
(2)\end{array}$ & 0.80 & 0.5 & $\begin{array}{c}3.5 \\
(1.75)\end{array}$ & 0.80 & 2 & 1.58 & A17 \\
\hline & $8-5 g-T 4.0-4 \# 4-i-3.5-3-9.5^{a}$ & $\begin{array}{l}\mathrm{A} \\
\mathrm{B}\end{array}$ & 8 & $\begin{array}{c}4 \\
(2) \\
\end{array}$ & 0.80 & 0.5 & $\begin{array}{c}3.5 \\
(1.75) \\
\end{array}$ & 0.80 & 2 & 1.58 & A17 \\
\hline \multirow{6}{*}{ Group 3} & $8-5-T 4.0-0-i-2.5-3-12.5^{a}$ & $\begin{array}{l}\text { A } \\
B\end{array}$ & - & - & - & 0.375 & $\begin{array}{c}3.5 \\
(1.75)\end{array}$ & 0.44 & 2 & 1.58 & A2 \\
\hline & $8-5-T 4.0-0-i-3.5-3-12.5^{a}$ & $\begin{array}{l}\text { A } \\
B\end{array}$ & - & - & - & 0.375 & $\begin{array}{c}3.5 \\
(1.75) \\
\end{array}$ & 0.44 & 2 & 1.58 & A2 \\
\hline & $8-5-T 4.0-5 \# 3-i-2.5-3-9.5^{a}$ & $\begin{array}{l}\text { A } \\
B\end{array}$ & 10 & $\begin{array}{c}3 \\
(1.5)\end{array}$ & 0.66 & 0.375 & $\begin{array}{c}3.5 \\
(1.75)\end{array}$ & 0.44 & 2 & 1.58 & A2 \\
\hline & $8-5-T 4.0-5 \# 3-i-3.5-3-9.5^{a}$ & $\begin{array}{l}\mathrm{A} \\
\mathrm{B}\end{array}$ & 10 & $\begin{array}{c}3 \\
(1.5) \\
\end{array}$ & 0.66 & 0.375 & $\begin{array}{c}3.5 \\
(1.75) \\
\end{array}$ & 0.44 & 2 & 1.58 & A2 \\
\hline & 8-5-T4.0-4\#4-i-2.5-3-9.5 & $\begin{array}{l}A \\
B\end{array}$ & 8 & $\begin{array}{c}4 \\
(2)\end{array}$ & 0.80 & 0.5 & $\begin{array}{c}3.5 \\
(1.75)\end{array}$ & 0.80 & 2 & 1.58 & A17 \\
\hline & $8-5-T 4.0-4 \# 4-i-3.5-3-9.5^{a}$ & $\begin{array}{l}\mathrm{A} \\
\mathrm{B}\end{array}$ & 8 & $\begin{array}{c}4 \\
(2) \\
\end{array}$ & 0.80 & 0.5 & $\begin{array}{c}3.5 \\
(1.75)\end{array}$ & 0.80 & 2 & 1.58 & A17 \\
\hline \multirow{3}{*}{ Group 4} & 8-8-F4.1-0-i-2.5-3-10.5 & $\begin{array}{l}\text { A } \\
B\end{array}$ & - & - & - & 0.375 & $\begin{array}{c}4 \\
(2) \\
\end{array}$ & 0.44 & 2 & 1.58 & A1 \\
\hline & 8-8-F4.1-2\#3-i-2.5-3-10 & $\begin{array}{l}\text { A } \\
\mathrm{B} \\
\end{array}$ & 4 & $\begin{array}{c}5 \\
(5.5) \\
\end{array}$ & 0.22 & 0.375 & $\begin{array}{c}4 \\
(2) \\
\end{array}$ & 0.44 & 2 & 1.58 & A1 \\
\hline & (3@3)8-8-F4.1-0-i-2.5-3-10.5 & $\begin{array}{l}\mathrm{A} \\
\mathrm{B} \\
\mathrm{C} \\
\end{array}$ & - & - & - & 0.375 & $\begin{array}{c}3.5 \\
(1.75)\end{array}$ & 0.44 & 3 & 2.37 & A3 \\
\hline
\end{tabular}

a Specimen contained crossties within joint region

${ }^{b}$ Specimen had no confining reinforcement above joint region

c Value in parenthesis is the spacing between the first hoop and the center of the headed bar 
Table A.3 Cont. Comprehensive test results and data for beam-column joint specimens

\begin{tabular}{|c|c|c|c|c|c|c|c|c|c|c|}
\hline & Specimen & Head & $\begin{array}{c}\text { Failure } \\
\text { Type }\end{array}$ & $\begin{array}{c}\text { Lead (Head) } \\
\text { Slip } \\
\text { in. }\end{array}$ & $\begin{array}{l}\boldsymbol{T}_{\max } \\
\text { kips }\end{array}$ & $\begin{array}{c}\boldsymbol{f}_{\text {su,max }} \\
\text { ksi }\end{array}$ & $\begin{array}{l}T_{\text {ind }} \\
\text { kips }\end{array}$ & $\begin{array}{l}\boldsymbol{T}_{\text {total }} \\
\text { kips }\end{array}$ & $\begin{array}{c}\boldsymbol{T} \\
\text { kips }\end{array}$ & $\begin{array}{l}\boldsymbol{f}_{\text {su }} \\
\mathrm{ksi}\end{array}$ \\
\hline \multirow{6}{*}{ Group 1} & $8-5-T 4.0-0-i-3-3-15.5^{\mathrm{a} b}$ & $\begin{array}{l}\text { A } \\
B\end{array}$ & $\mathrm{SB} / \mathrm{FP}$ & $\begin{array}{c}0.019 \\
-\end{array}$ & $\begin{array}{l}81.6 \\
92.2\end{array}$ & $\begin{array}{l}103.3 \\
116.7\end{array}$ & $\begin{array}{l}81.6 \\
79.3\end{array}$ & 160.9 & 80.4 & 101.8 \\
\hline & $8-5-T 4.0-0-i-4-3-15.5^{a}$ & $\begin{array}{l}\text { A } \\
\text { B }\end{array}$ & $\mathrm{SB} / \mathrm{FP}$ & $\begin{array}{c}0.308 \\
-\end{array}$ & $\begin{array}{l}92.5 \\
98.5\end{array}$ & $\begin{array}{l}117.1 \\
124.7\end{array}$ & $\begin{array}{l}92.5 \\
98.4\end{array}$ & 190.9 & 95.4 & 120.8 \\
\hline & $8-5-T 4.0-4 \# 3-i-3-3-12.5^{\text {a c }}$ & $\begin{array}{l}\mathrm{A} \\
\mathrm{B}\end{array}$ & $\mathrm{SB} / \mathrm{FP}$ & $\begin{array}{c}0.227 \\
-\end{array}$ & $\begin{array}{c}87.9 \\
-^{\ddagger}\end{array}$ & $\begin{array}{c}111.3 \\
-^{\ddagger}\end{array}$ & $\begin{array}{l}87.9 \\
87.1\end{array}$ & 175.0 & 87.5 & 110.8 \\
\hline & $8-5-T 4.0-4 \# 3-i-4-3-12.5^{a}$ & $\begin{array}{l}\text { A } \\
\text { B }\end{array}$ & $\mathrm{SB} / \mathrm{FP}$ & $\begin{array}{c}- \\
0.239\end{array}$ & $\begin{array}{c}110.8 \\
95.2\end{array}$ & $\begin{array}{l}140.3 \\
120.5\end{array}$ & $\begin{array}{l}97.2 \\
95.1\end{array}$ & 192.3 & 96.2 & 121.7 \\
\hline & $8-5-T 4.0-4 \# 4-i-3-3-12.5^{a}$ & $\begin{array}{l}\mathrm{A} \\
\mathrm{B}\end{array}$ & $\mathrm{SB} / \mathrm{FP}$ & $\begin{array}{c}0.049 \\
-\end{array}$ & $\begin{array}{l}109.5 \\
111.0\end{array}$ & $\begin{array}{l}138.6 \\
140.5\end{array}$ & $\begin{array}{l}109.4 \\
108.6\end{array}$ & 218.1 & 109.0 & 138.0 \\
\hline & $8-5-T 4.0-4 \# 4-\mathrm{i}-4-3-12.5^{\mathrm{a}}$ & $\begin{array}{l}\mathrm{A} \\
\mathrm{B}\end{array}$ & $\mathrm{SB} / \mathrm{FP}$ & $\begin{array}{l}0.228 \\
0.350\end{array}$ & $\begin{array}{l}102.5 \\
103.4\end{array}$ & $\begin{array}{l}129.7 \\
130.9\end{array}$ & $\begin{array}{l}102.5 \\
100.5\end{array}$ & 203.0 & 101.5 & 128.5 \\
\hline \multirow{6}{*}{ Group 2} & $8-5 g-T 4.0-0-i-2.5-3-12.5^{a}$ & $\begin{array}{l}\mathrm{A} \\
\mathrm{B}\end{array}$ & $\mathrm{SB} / \mathrm{FP}$ & $\begin{array}{c}- \\
0.022(0.008)\end{array}$ & $\begin{array}{c}117.6 \\
96.1 \\
\end{array}$ & $\begin{array}{l}148.9 \\
121.6 \\
\end{array}$ & $\begin{array}{l}99.3 \\
96.1 \\
\end{array}$ & 195.4 & 97.7 & 123.7 \\
\hline & $8-5 g-T 4.0-0-i-3.5-3-12.5^{a}$ & $\begin{array}{l}\text { A } \\
\text { B }\end{array}$ & $\mathrm{SB} / \mathrm{FP}$ & $\begin{array}{c}0.427 \\
(0.025)\end{array}$ & $\begin{array}{c}104.6 \\
93.6\end{array}$ & $\begin{array}{l}132.4 \\
118.5\end{array}$ & $\begin{array}{l}93.2 \\
93.6\end{array}$ & 186.8 & 93.4 & 118.2 \\
\hline & $8-5 g-T 4.0-5 \# 3-i-2.5-3-9.5^{a}$ & $\begin{array}{l}\text { A } \\
B\end{array}$ & SB & $\begin{array}{l}0.190 \\
0.545\end{array}$ & $\begin{array}{l}78.9 \\
92.6\end{array}$ & $\begin{array}{c}99.9 \\
117.2\end{array}$ & $\begin{array}{l}78.9 \\
78.5\end{array}$ & 157.4 & 78.7 & 99.6 \\
\hline & $8-5 g-T 4.0-5 \# 3-i-3.5-3-9.5^{a}$ & $\begin{array}{l}\text { A } \\
B\end{array}$ & SB & $\begin{array}{l}0.599 \\
0.193\end{array}$ & $\begin{array}{l}88.4 \\
78.7\end{array}$ & $\begin{array}{l}111.9 \\
99.6\end{array}$ & $\begin{array}{l}80.3 \\
78.7\end{array}$ & 159.0 & 79.5 & 100.6 \\
\hline & $8-5 g-T 4.0-4 \# 4-i-2.5-3-9.5^{a}$ & $\begin{array}{l}\text { A } \\
\text { B }\end{array}$ & SB & $\begin{array}{c}0.187 \\
0.498(0.032)\end{array}$ & $\begin{array}{c}92.2 \\
102.2\end{array}$ & $\begin{array}{l}116.7 \\
129.4\end{array}$ & $\begin{array}{l}92.2 \\
89.3\end{array}$ & 181.5 & 90.7 & 114.8 \\
\hline & $8-5 g-T 4.0-4 \# 4-i-3.5-3-9.5^{a}$ & $\begin{array}{l}\mathrm{A} \\
\mathrm{B}\end{array}$ & SB & $\begin{array}{c}- \\
(0.056)\end{array}$ & $\begin{array}{c}112.0 \\
95.8\end{array}$ & $\begin{array}{l}141.8 \\
121.3\end{array}$ & $\begin{array}{l}97.6 \\
95.8\end{array}$ & 193.4 & 96.7 & 122.4 \\
\hline \multirow{6}{*}{ Group 3} & $8-5-T 4 \cdot 0-0-i-2.5-3-12.5^{a}$ & $\begin{array}{l}\mathrm{A} \\
\mathrm{B}\end{array}$ & $\mathrm{SB} / \mathrm{FP}$ & $\begin{array}{l}- \\
-\end{array}$ & $\begin{array}{l}84.0 \\
95.0 \\
\end{array}$ & $\begin{array}{l}106.3 \\
120.3 \\
\end{array}$ & $\begin{array}{l}84.0 \\
82.6 \\
\end{array}$ & 166.6 & 83.3 & 105.4 \\
\hline & $8-5-T 4.0-0-i-3.5-3-12.5^{a}$ & $\begin{array}{l}\mathrm{A} \\
\mathrm{B}\end{array}$ & $\mathrm{SB} / \mathrm{FP}$ & $\begin{array}{c}0.013 \\
-\end{array}$ & $\begin{array}{c}92.1 \\
100.1\end{array}$ & $\begin{array}{l}116.6 \\
126.7\end{array}$ & $\begin{array}{l}92.1 \\
91.6\end{array}$ & 183.7 & 91.9 & 116.3 \\
\hline & $8-5-T 4.0-5 \# 3-i-2.5-3-9.5^{a}$ & $\begin{array}{l}\mathrm{A} \\
\mathrm{B}\end{array}$ & SB & $\begin{array}{l}0.185 \\
0.163 \\
\end{array}$ & $\begin{array}{l}74.5 \\
74.0 \\
\end{array}$ & $\begin{array}{l}94.3 \\
93.7 \\
\end{array}$ & $\begin{array}{l}74.5 \\
74.0 \\
\end{array}$ & 148.5 & 74.2 & 93.9 \\
\hline & $8-5-T 4.0-5 \# 3-i-3.5-3-9.5^{a}$ & $\begin{array}{l}\mathrm{A} \\
\mathrm{B}\end{array}$ & $\mathrm{SB} / \mathrm{FP}$ & $\begin{array}{c}- \\
0.570\end{array}$ & $\begin{array}{l}80.7 \\
96.1\end{array}$ & $\begin{array}{l}102.2 \\
121.6\end{array}$ & $\begin{array}{l}80.7 \\
80.4 \\
\end{array}$ & 161.1 & 80.6 & 102.0 \\
\hline & $8-5-T 4.0-4 \# 4-i-2.5-3-9.5^{a}$ & $\begin{array}{l}\text { A } \\
B\end{array}$ & $\mathrm{SB} / \mathrm{FP}$ & $\begin{array}{c}- \\
(0.005)\end{array}$ & $\begin{array}{l}94.9 \\
89.5\end{array}$ & $\begin{array}{l}120.1 \\
113.3\end{array}$ & $\begin{array}{l}91.6 \\
89.5\end{array}$ & 181.1 & 90.5 & 114.6 \\
\hline & $8-5-T 4.0-4 \# 4-i-3.5-3-9.5^{a}$ & $\begin{array}{l}A \\
B\end{array}$ & $\mathrm{SB} / \mathrm{FP}$ & $\begin{array}{c}0.186 \\
- \\
\end{array}$ & $\begin{array}{l}86.6 \\
89.0\end{array}$ & $\begin{array}{l}109.6 \\
112.7 \\
\end{array}$ & $\begin{array}{l}86.6 \\
84.6\end{array}$ & 171.1 & 85.6 & 108.4 \\
\hline \multirow{3}{*}{ Group 4} & 8-8-F4.1-0-i-2.5-3-10.5 & $\begin{array}{l}\text { A } \\
\text { B }\end{array}$ & CB & $\begin{array}{l}- \\
- \\
\end{array}$ & $\begin{array}{l}77.8 \\
76.3 \\
\end{array}$ & $\begin{array}{l}98.5 \\
96.6 \\
\end{array}$ & $\begin{array}{l}77.8 \\
76.3 \\
\end{array}$ & 154.1 & 77.1 & 97.6 \\
\hline & 8-8-F4.1-2\#3-i-2.5-3-10 & $\begin{array}{l}\mathrm{A} \\
\mathrm{B}\end{array}$ & CB & $\begin{array}{l}0.107 \\
0.168 \\
\end{array}$ & $\begin{array}{l}73.5 \\
73.3 \\
\end{array}$ & $\begin{array}{l}93.0 \\
92.8 \\
\end{array}$ & $\begin{array}{l}73.5 \\
73.3 \\
\end{array}$ & 146.8 & 73.4 & 92.9 \\
\hline & (3@3)8-8-F4.1-0-i-2.5-3-10.5 & $\begin{array}{l}\text { A } \\
\text { B } \\
\text { C }\end{array}$ & CB & $\begin{array}{l}0.170 \\
0.212 \\
0.162\end{array}$ & $\begin{array}{l}49.0 \\
56.2 \\
59.1\end{array}$ & $\begin{array}{l}62.0 \\
71.1 \\
74.8\end{array}$ & $\begin{array}{l}49.0 \\
56.2 \\
59.1\end{array}$ & 164.3 & 54.8 & 69.4 \\
\hline
\end{tabular}

a Specimen contained crossties within joint region

b Specimen had no confining reinforcement above joint region

${ }^{\ddagger}$ No anchorage failure on the bar 
Table A.3 Cont. Comprehensive test results and data for beam-column joint specimens

\begin{tabular}{|c|c|c|c|c|c|c|c|c|c|c|}
\hline & Specimen & Head & $\begin{array}{l}c_{o} \\
\text { in. }\end{array}$ & $A_{b r g}$ & $\begin{array}{l}\ell_{\text {eh }} \\
\text { in. }\end{array}$ & $\begin{array}{c}\ell \text { eh,avg } \\
\text { in. }\end{array}$ & $\begin{array}{l}\boldsymbol{f}_{\boldsymbol{c m}} \\
\mathrm{psi}\end{array}$ & $\begin{array}{l}\text { Age } \\
\text { days }\end{array}$ & $\begin{array}{l}\boldsymbol{d}_{\boldsymbol{b}} \\
\text { in. }\end{array}$ & $\begin{array}{l}\boldsymbol{A}_{\boldsymbol{b}} \\
\text { in. }^{2}\end{array}$ \\
\hline \multirow{10}{*}{ Group 4} & (3@3)8-8-F4.1-0-i-2.5-3-10.5-HP & $\begin{array}{l}\text { A } \\
\text { B } \\
\text { C }\end{array}$ & 2.0 & $4.1 A_{b}$ & $\begin{array}{l}10.13 \\
10.13 \\
10.75\end{array}$ & 10.33 & 8450 & 9 & 1 & 0.79 \\
\hline & (3@3)8-8-F4.1-2\#3-i-2.5-3-10 & $\begin{array}{l}\text { A } \\
\text { B } \\
\text { C }\end{array}$ & 2.0 & $4.1 A_{b}$ & $\begin{array}{l}10.13 \\
10.00 \\
10.13\end{array}$ & 10.08 & 8260 & 8 & 1 & 0.79 \\
\hline & (3@3)8-8-F4.1-2\#3-i-2.5-3-10-HP & $\begin{array}{l}\text { A } \\
B \\
C\end{array}$ & 2.0 & $4.1 A_{b}$ & $\begin{array}{l}10.25 \\
10.13 \\
10.50\end{array}$ & 10.29 & 8260 & 8 & 1 & 0.79 \\
\hline & (3@4)8-8-F4.1-0-i-2.5-3-10.5 & $\begin{array}{l}\text { A } \\
B \\
C\end{array}$ & 2.0 & $4.1 A_{b}$ & $\begin{array}{l}10.88 \\
10.75 \\
10.88\end{array}$ & 10.83 & 8450 & 9 & 1 & 0.79 \\
\hline & (3@4)8-8-F4.1-2\#3-i-2.5-3-10 & $\begin{array}{l}\mathrm{A} \\
\mathrm{B} \\
\mathrm{C}\end{array}$ & 2.0 & $4.1 A_{b}$ & $\begin{array}{c}9.75 \\
9.63 \\
10.25 \\
\end{array}$ & 9.88 & 8050 & 7 & 1 & 0.79 \\
\hline & (3@4)8-8-F4.1-2\#3-i-2.5-3-10-HP & $\begin{array}{l}\text { A } \\
B \\
C\end{array}$ & 2.0 & $4.1 A_{b}$ & $\begin{array}{l}10.00 \\
10.75 \\
10.25\end{array}$ & 10.33 & 8050 & 7 & 1 & 0.79 \\
\hline & (3@5)8-8-F4.1-0-i-2.5-3-10.5 & $\begin{array}{l}\text { A } \\
B \\
C \\
\end{array}$ & 2.0 & $4.1 A_{b}$ & $\begin{array}{l}10.50 \\
10.38 \\
10.19 \\
\end{array}$ & 10.35 & 8050 & 7 & 1 & 0.79 \\
\hline & (3@5)8-8-F4.1-0-i-2.5-3-10.5-HP & $\begin{array}{l}A \\
B \\
C\end{array}$ & 2.0 & $4.1 A_{b}$ & $\begin{array}{c}9.75 \\
10.50 \\
10.50\end{array}$ & 10.25 & 8260 & 8 & 1 & 0.79 \\
\hline & (3@5)8-8-F4.1-2\#3-i-2.5-3-10.5 & $\begin{array}{l}\text { A } \\
\text { B } \\
\text { C } \\
\end{array}$ & 2.0 & $4.1 A_{b}$ & $\begin{array}{c}9.63 \\
9.75 \\
10.00 \\
\end{array}$ & 9.79 & 8260 & 8 & 1 & 0.79 \\
\hline & (3@5)8-8-F4.1-2\#3-i-2.5-3-10.5-HP & $\begin{array}{l}\mathrm{A} \\
\mathrm{B} \\
\mathrm{C}\end{array}$ & 2.0 & $4.1 A_{b}$ & $\begin{array}{c}9.88 \\
10.00 \\
10.13 \\
\end{array}$ & 10.00 & 8260 & 8 & 1 & 0.79 \\
\hline \multirow{5}{*}{ Group 5} & 8-12-F4.1-0-i-2.5-3-10 & $\begin{array}{l}\text { A } \\
\text { B }\end{array}$ & 2.0 & $4.1 A_{b}$ & $\begin{array}{l}9.63 \\
9.75 \\
\end{array}$ & 9.69 & 11760 & 34 & 1 & 0.79 \\
\hline & 8-12-F4.1-5\#3-i-2.5-3-10 & $\begin{array}{l}\text { A } \\
\text { B }\end{array}$ & 2.0 & $4.1 A_{b}$ & $\begin{array}{l}10.00 \\
10.00 \\
\end{array}$ & 10.00 & 11760 & 34 & 1 & 0.79 \\
\hline & (3@3)8-12-F4.1-0-i-2.5-3-10 & $\begin{array}{l}\text { A } \\
B \\
\text { C }\end{array}$ & 2.0 & $4.1 A_{b}$ & $\begin{array}{c}9.81 \\
10.00 \\
9.88 \\
\end{array}$ & 9.90 & 11040 & 31 & 1 & 0.79 \\
\hline & (3@3)8-12-F4.1-5\#3-i-2.5-3-10 & $\begin{array}{l}\text { A } \\
\text { B } \\
\text { C }\end{array}$ & 2.0 & $4.1 A_{b}$ & $\begin{array}{c}10.00 \\
10.13 \\
9.88 \\
\end{array}$ & 10.00 & 11040 & 31 & 1 & 0.79 \\
\hline & (3@4)8-12-F4.1-0-i-2.5-3-10 & $\begin{array}{l}\text { A } \\
\text { B } \\
\text { C }\end{array}$ & 2.0 & $4.1 A_{b}$ & $\begin{array}{c}10.00 \\
9.75 \\
10.00\end{array}$ & 9.92 & 11440 & 32 & 1 & 0.79 \\
\hline
\end{tabular}


Table A.3 Cont. Comprehensive test results and data for beam-column joint specimens

\begin{tabular}{|c|c|c|c|c|c|c|c|c|c|c|c|c|}
\hline & Specimen & Head & $\begin{array}{c}\boldsymbol{b} \\
\text { in. }\end{array}$ & $\begin{array}{c}\boldsymbol{h} \\
\text { in. }\end{array}$ & $\begin{array}{l}\boldsymbol{h}_{\boldsymbol{c l}} \\
\text { in. }\end{array}$ & $\begin{array}{l}\boldsymbol{d}_{\text {eff }} \\
\text { in. }\end{array}$ & $\begin{array}{l}c_{s o} \\
\text { in. }\end{array}$ & $\begin{array}{c}\boldsymbol{C}_{\boldsymbol{s o}, \text { avg }} \\
\text { in. }\end{array}$ & $\begin{array}{l}c_{b c} \\
\text { in. }\end{array}$ & $\begin{array}{l}\boldsymbol{c}_{\boldsymbol{c h}} \\
\text { in. }\end{array}$ & $\begin{array}{l}\boldsymbol{d}_{t r} \\
\text { in. }\end{array}$ & $\begin{array}{l}\boldsymbol{A}_{t r, \boldsymbol{l}} \\
\text { in. }^{2}\end{array}$ \\
\hline \multirow{10}{*}{ Group 4} & (3@3)8-8-F4.1-0-i-2.5-3-10.5-HP & $\begin{array}{l}\text { A } \\
\text { B } \\
\text { C }\end{array}$ & 11.8 & 14.6 & 10.25 & 12.95 & $\begin{array}{c}2.5 \\
- \\
2.5\end{array}$ & 2.5 & $\begin{array}{l}3.4 \\
3.4 \\
2.8\end{array}$ & $\begin{array}{l}3.0 \\
2.8\end{array}$ & - & - \\
\hline & (3@3)8-8-F4.1-2\#3-i-2.5-3-10 & $\begin{array}{l}\text { A } \\
\text { B } \\
\text { C }\end{array}$ & 11.8 & 14.2 & 10.25 & 13.64 & $\begin{array}{c}2.5 \\
- \\
2.4\end{array}$ & 2.4 & $\begin{array}{l}3.1 \\
3.2 \\
3.1\end{array}$ & $\begin{array}{l}2.9 \\
3.0\end{array}$ & 0.375 & 0.11 \\
\hline & (3@3)8-8-F4.1-2\#3-i-2.5-3-10-HP & $\begin{array}{l}\text { A } \\
\text { B } \\
\text { C }\end{array}$ & 12.1 & 14.0 & 10.25 & 13.36 & $\begin{array}{c}2.5 \\
- \\
2.6\end{array}$ & 2.6 & $\begin{array}{l}2.8 \\
2.9 \\
2.5\end{array}$ & $\begin{array}{l}3.0 \\
3.0\end{array}$ & 0.375 & 0.11 \\
\hline & (3@4)8-8-F4.1-0-i-2.5-3-10.5 & $\begin{array}{l}\text { A } \\
\text { B } \\
\text { C }\end{array}$ & 13.9 & 14.7 & 10.25 & 12.94 & $\begin{array}{c}2.5 \\
- \\
2.5\end{array}$ & 2.5 & $\begin{array}{l}2.8 \\
3.0 \\
2.8\end{array}$ & $\begin{array}{l}3.9 \\
4.0\end{array}$ & - & - \\
\hline & (3@4)8-8-F4.1-2\#3-i-2.5-3-10 & $\begin{array}{l}\text { A } \\
\text { B } \\
\text { C }\end{array}$ & 13.8 & 14.2 & 10.25 & 12.92 & $\begin{array}{c}2.4 \\
- \\
2.4 \\
\end{array}$ & 2.4 & $\begin{array}{l}3.4 \\
3.5 \\
2.9 \\
\end{array}$ & $\begin{array}{l}4.0 \\
4.0 \\
\end{array}$ & 0.375 & 0.11 \\
\hline & (3@4)8-8-F4.1-2\#3-i-2.5-3-10-HP & $\begin{array}{l}\text { A } \\
\text { B } \\
\text { C }\end{array}$ & 14.3 & 14.8 & 10.25 & 13.61 & $\begin{array}{c}2.5 \\
- \\
2.5 \\
\end{array}$ & 2.5 & $\begin{array}{l}3.8 \\
3.0 \\
3.5\end{array}$ & $\begin{array}{l}4.0 \\
4.3\end{array}$ & 0.375 & 0.11 \\
\hline & (3@5)8-8-F4.1-0-i-2.5-3-10.5 & $\begin{array}{l}\text { A } \\
\text { B } \\
\text { C }\end{array}$ & 15.9 & 14.7 & 10.25 & 12.95 & $\begin{array}{c}2.5 \\
- \\
2.4\end{array}$ & 2.5 & $\begin{array}{l}3.2 \\
3.3 \\
3.5\end{array}$ & $\begin{array}{l}4.9 \\
5.1 \\
\end{array}$ & - & - \\
\hline & (3@5)8-8-F4.1-0-i-2.5-3-10.5-HP & $\begin{array}{l}\text { A } \\
\text { B } \\
\text { C }\end{array}$ & 16.0 & 14.8 & 10.25 & 12.71 & $\begin{array}{c}2.4 \\
- \\
2.5 \\
\end{array}$ & 2.4 & $\begin{array}{l}4.0 \\
3.3 \\
3.3 \\
\end{array}$ & $\begin{array}{l}5.1 \\
5.0 \\
\end{array}$ & - & - \\
\hline & (3@5)8-8-F4.1-2\#3-i-2.5-3-10.5 & $\begin{array}{l}\text { A } \\
B \\
C\end{array}$ & 15.3 & 14.2 & 10.25 & 12.55 & $\begin{array}{c}2.4 \\
- \\
2.4 \\
\end{array}$ & 2.4 & $\begin{array}{l}3.6 \\
3.4 \\
3.2 \\
\end{array}$ & $\begin{array}{l}4.8 \\
4.8 \\
\end{array}$ & 0.375 & 0.11 \\
\hline & (3@5)8-8-F4.1-2\#3-i-2.5-3-10.5-HP & $\begin{array}{l}\text { A } \\
B \\
C\end{array}$ & 16.0 & 14.1 & 10.25 & 12.94 & $\begin{array}{c}2.4 \\
- \\
2.5 \\
\end{array}$ & 2.4 & $\begin{array}{l}3.2 \\
3.1 \\
2.9 \\
\end{array}$ & $\begin{array}{l}5.0 \\
5.1 \\
\end{array}$ & 0.375 & 0.11 \\
\hline \multirow{5}{*}{ Group 5} & $8-12-F 4.1-0-i-2.5-3-10$ & $\begin{array}{l}\text { A } \\
\text { B }\end{array}$ & 16.9 & 14.2 & 10.25 & 11.55 & $\begin{array}{l}2.4 \\
2.6 \\
\end{array}$ & 2.5 & $\begin{array}{l}3.5 \\
3.4 \\
\end{array}$ & 10.9 & 0.375 & - \\
\hline & 8-12-F4.1-5\#3-i-2.5-3-10 & $\begin{array}{l}\text { A } \\
\text { B }\end{array}$ & 17.0 & 14.2 & 10.25 & 11.83 & $\begin{array}{l}2.5 \\
2.5 \\
\end{array}$ & 2.5 & $\begin{array}{l}3.2 \\
3.2 \\
\end{array}$ & 11.0 & 0.375 & 0.11 \\
\hline & (3@3)8-12-F4.1-0-i-2.5-3-10 & $\begin{array}{l}\text { A } \\
\text { B } \\
\text { C }\end{array}$ & 12.0 & 14.2 & 10.25 & 11.98 & $\begin{array}{c}2.5 \\
- \\
2.4\end{array}$ & 2.4 & $\begin{array}{l}3.3 \\
3.2 \\
3.3\end{array}$ & $\begin{array}{l}3.1 \\
3.0\end{array}$ & 0.375 & - \\
\hline & (3@3)8-12-F4.1-5\#3-i-2.5-3-10 & $\begin{array}{l}\text { A } \\
\text { B } \\
\text { C }\end{array}$ & 12.0 & 13.9 & 10.25 & 12.81 & $\begin{array}{c}2.4 \\
- \\
2.4 \\
\end{array}$ & 2.4 & $\begin{array}{l}2.9 \\
2.8 \\
3.1 \\
\end{array}$ & $\begin{array}{l}3.0 \\
3.1 \\
\end{array}$ & 0.375 & 0.11 \\
\hline & (3@4)8-12-F4.1-0-i-2.5-3-10 & $\begin{array}{l}\text { A } \\
\text { B } \\
\text { C }\end{array}$ & 14.0 & 14.0 & 10.25 & 11.91 & $\begin{array}{c}2.5 \\
- \\
2.5\end{array}$ & 2.5 & $\begin{array}{l}3.0 \\
3.3 \\
3.0\end{array}$ & $\begin{array}{l}4.0 \\
4.0\end{array}$ & 0.375 & - \\
\hline
\end{tabular}


Table A.3 Cont. Comprehensive test results and data for beam-column joint specimens

\begin{tabular}{|c|c|c|c|c|c|c|c|c|c|c|c|}
\hline & Specimen & Head & $N$ & $\begin{array}{l}\boldsymbol{s t r}^{*} \\
\text { in. }\end{array}$ & $\begin{array}{l}A_{t t} \\
\text { in. }^{2}\end{array}$ & $\begin{array}{l}\boldsymbol{d}_{\text {tro }} \\
\text { in. }\end{array}$ & $\begin{array}{l}\text { Stro }^{*} \\
\text { in. }\end{array}$ & $\begin{array}{l}A_{a b} \\
\text { in. }^{2}\end{array}$ & $n$ & $\begin{array}{l}A_{h s} \\
\text { in. }^{2}\end{array}$ & $\begin{array}{l}\text { Long. } \\
\text { Reinf. } \\
\text { Layout }\end{array}$ \\
\hline \multirow{10}{*}{ Group 4} & (3@3)8-8-F4.1-0-i-2.5-3-10.5-HP & $\begin{array}{l}\text { A } \\
\text { B } \\
\text { C }\end{array}$ & - & - & - & 0.375 & $\begin{array}{c}3.5 \\
(1.75)\end{array}$ & 0.44 & 3 & 2.37 & A3 \\
\hline & (3@3)8-8-F4.1-2\#3-i-2.5-3-10 & $\begin{array}{l}\text { A } \\
\text { B } \\
\text { C }\end{array}$ & 4 & $\begin{array}{c}5 \\
(5.5)\end{array}$ & 0.22 & 0.375 & $\begin{array}{c}3 \\
(1.5)\end{array}$ & 0.66 & 3 & 2.37 & A3 \\
\hline & (3@3)8-8-F4.1-2\#3-i-2.5-3-10-HP & $\begin{array}{l}\mathrm{A} \\
\mathrm{B} \\
\mathrm{C}\end{array}$ & 4 & $\begin{array}{c}5 \\
(5.5)\end{array}$ & 0.22 & 0.375 & $\begin{array}{c}3 \\
(1.5)\end{array}$ & 0.66 & 3 & 2.37 & A3 \\
\hline & (3@4)8-8-F4.1-0-i-2.5-3-10.5 & $\begin{array}{l}\text { A } \\
\text { B } \\
\text { C }\end{array}$ & - & - & - & 0.375 & $\begin{array}{c}4 \\
(2)\end{array}$ & 0.44 & 3 & 2.37 & A3 \\
\hline & (3@4)8-8-F4.1-2\#3-i-2.5-3-10 & $\begin{array}{l}\text { A } \\
B \\
C\end{array}$ & 4 & $\begin{array}{c}5 \\
(5.5)\end{array}$ & 0.22 & 0.375 & $\begin{array}{c}3.5 \\
(1.75)\end{array}$ & 0.44 & 3 & 2.37 & A3 \\
\hline & (3@4)8-8-F4.1-2\#3-i-2.5-3-10-HP & $\begin{array}{l}\text { A } \\
B \\
C\end{array}$ & 4 & $\begin{array}{c}5 \\
(5.5)\end{array}$ & 0.22 & 0.375 & $\begin{array}{c}3.5 \\
(1.75)\end{array}$ & 0.44 & 3 & 2.37 & A3 \\
\hline & (3@5)8-8-F4.1-0-i-2.5-3-10.5 & $\begin{array}{l}\mathrm{A} \\
\mathrm{B} \\
\mathrm{C}\end{array}$ & - & - & - & 0.375 & $\begin{array}{c}4 \\
(2)\end{array}$ & 0.44 & 3 & 2.37 & A3 \\
\hline & (3@5)8-8-F4.1-0-i-2.5-3-10.5-HP & $\begin{array}{l}\mathrm{A} \\
\mathrm{B} \\
\mathrm{C}\end{array}$ & - & - & - & 0.375 & $\begin{array}{c}4 \\
(2)\end{array}$ & 0.44 & 3 & 2.37 & A3 \\
\hline & (3@5)8-8-F4.1-2\#3-i-2.5-3-10.5 & $\begin{array}{l}\text { A } \\
B \\
C\end{array}$ & 4 & $\begin{array}{c}5 \\
(5.5)\end{array}$ & 0.22 & 0.375 & $\begin{array}{c}4 \\
(2)\end{array}$ & 0.44 & 3 & 2.37 & A3 \\
\hline & (3@5)8-8-F4.1-2\#3-i-2.5-3-10.5-HP & $\begin{array}{l}\text { A } \\
B \\
C\end{array}$ & 4 & $\begin{array}{c}5 \\
(5.5)\end{array}$ & 0.22 & 0.375 & $\begin{array}{c}4 \\
(2)\end{array}$ & 0.44 & 3 & 2.37 & A3 \\
\hline \multirow{5}{*}{ Group 5} & 8-12-F4.1-0-i-2.5-3-10 & $\begin{array}{l}\mathrm{A} \\
\mathrm{B} \\
\end{array}$ & - & - & - & 0.375 & $\begin{array}{c}4 \\
(2) \\
\end{array}$ & 0.44 & 2 & 1.58 & A4 \\
\hline & 8-12-F4.1-5\#3-i-2.5-3-10 & $\begin{array}{l}\text { A } \\
\text { B }\end{array}$ & 10 & $\begin{array}{c}3 \\
(1.5) \\
\end{array}$ & 0.66 & 0.375 & $\begin{array}{c}4 \\
(2) \\
\end{array}$ & 0.44 & 2 & 1.58 & A5 \\
\hline & (3@3)8-12-F4.1-0-i-2.5-3-10 & $\begin{array}{l}\text { A } \\
\mathrm{B} \\
\mathrm{C}\end{array}$ & - & - & - & 0.375 & $\begin{array}{c}3 \\
(1.5)\end{array}$ & 0.66 & 3 & 2.37 & A5 \\
\hline & (3@3)8-12-F4.1-5\#3-i-2.5-3-10 & $\begin{array}{l}\text { A } \\
B \\
C\end{array}$ & 10 & $\begin{array}{c}3 \\
(1.5)\end{array}$ & 0.66 & 0.375 & $\begin{array}{c}3 \\
(1.5)\end{array}$ & 0.66 & 3 & 2.37 & A5 \\
\hline & (3@4)8-12-F4.1-0-i-2.5-3-10 & $\begin{array}{l}\text { A } \\
\text { B } \\
\text { C }\end{array}$ & - & - & - & 0.375 & $\begin{array}{c}3 \\
(1.5)\end{array}$ & 0.66 & 3 & 2.37 & A5 \\
\hline
\end{tabular}

${ }^{*}$ Value in parenthesis is the spacing between the first hoop and the center of the headed bar 
Table A.3 Cont. Comprehensive test results and data for beam-column joint specimens

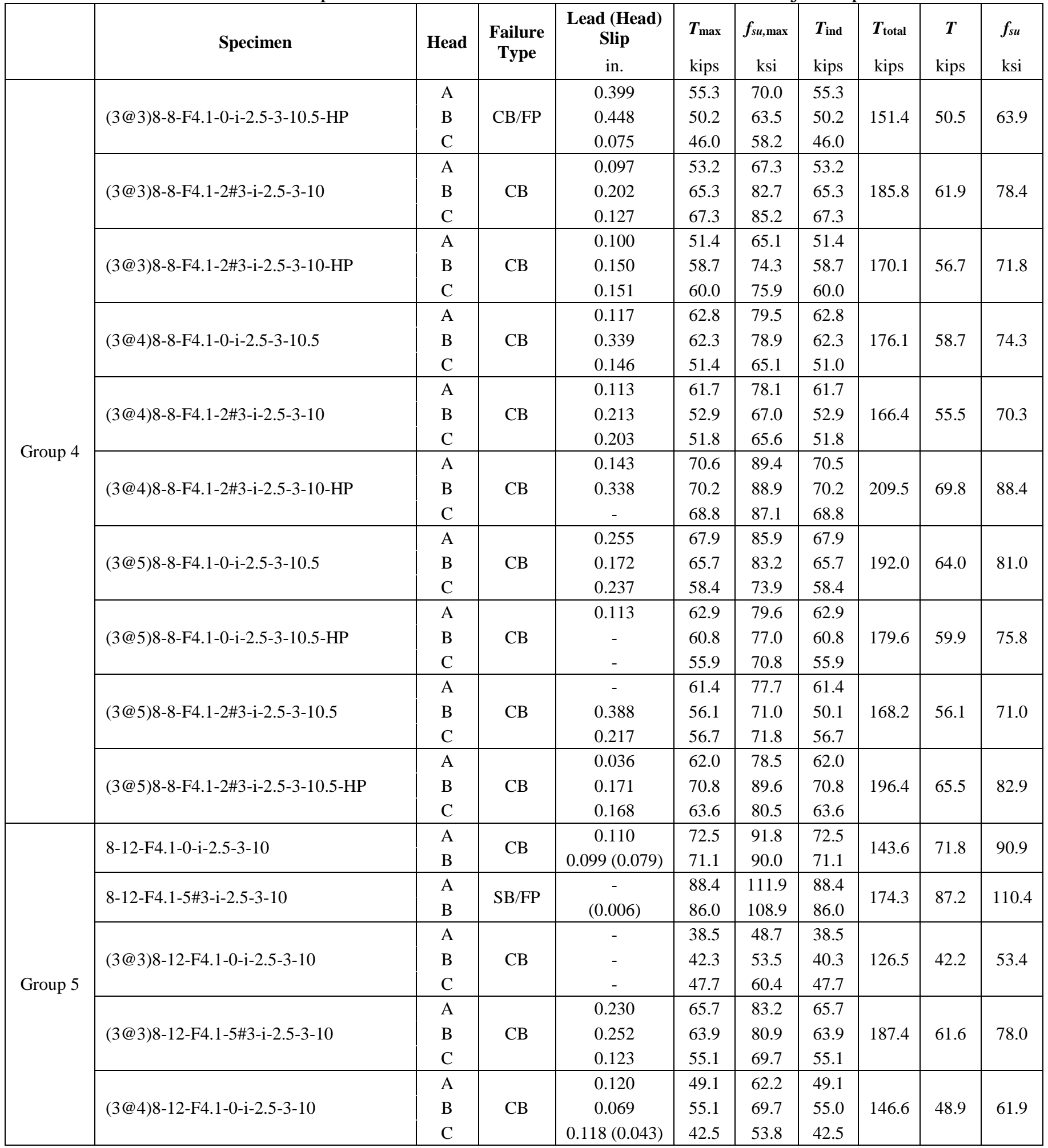


Table A.3 Cont. Comprehensive test results and data for beam-column joint specimens

\begin{tabular}{|c|c|c|c|c|c|c|c|c|c|c|}
\hline & Specimen & Head & $\begin{array}{l}\boldsymbol{c}_{\boldsymbol{o}} \\
\text { in. }\end{array}$ & $A_{b r g}$ & $\begin{array}{l}\ell_{\text {eh }} \\
\text { in. }\end{array}$ & $\begin{array}{c}\text { leh,avg } \\
\text { in. }\end{array}$ & $\begin{array}{l}\boldsymbol{f}_{\boldsymbol{c m}} \\
\mathrm{psi}\end{array}$ & $\begin{array}{l}\text { Age } \\
\text { days }\end{array}$ & $\begin{array}{l}\boldsymbol{d}_{\boldsymbol{b}} \\
\text { in. }\end{array}$ & $\begin{array}{l}\boldsymbol{A}_{\boldsymbol{b}} \\
\text { in. }^{2}\end{array}$ \\
\hline \multirow{3}{*}{ Group 5} & (3@4)8-12-F4.1-5\#3-i-2.5-3-10 & $\begin{array}{l}\text { A } \\
\text { B } \\
\text { C }\end{array}$ & 2.0 & $4.1 A_{b}$ & $\begin{array}{l}9.81 \\
9.88 \\
9.63\end{array}$ & 9.77 & 11440 & 32 & 1 & 0.79 \\
\hline & (3@5)8-12-F4.1-0-i-2.5-3-10 & $\begin{array}{l}\text { A } \\
B \\
C\end{array}$ & 2.0 & $4.1 A_{b}$ & $\begin{array}{c}9.88 \\
10.13 \\
9.75\end{array}$ & 9.92 & 11460 & 33 & 1 & 0.79 \\
\hline & (3@5)8-12-F4.1-5\#3-i-2.5-3-10 & $\begin{array}{l}\text { A } \\
B \\
C\end{array}$ & 2.0 & $4.1 A_{b}$ & $\begin{array}{l}9.75 \\
9.38 \\
9.69\end{array}$ & 9.60 & 11460 & 33 & 1 & 0.79 \\
\hline \multirow{12}{*}{ Group 6} & 8-5-S6.5-0-i-2.5-3-11.25 & $\begin{array}{l}\text { A } \\
B\end{array}$ & 1.8 & $6.5 A_{b}$ & $\begin{array}{l}11.00 \\
11.13\end{array}$ & 11.06 & 5500 & 6 & 1 & 0.79 \\
\hline & 8-5-S6.5-0-i-2.5-3-14.25 & $\begin{array}{l}\text { A } \\
B\end{array}$ & 1.8 & $6.5 A_{b}$ & $\begin{array}{l}14.38 \\
14.13\end{array}$ & 14.25 & 5500 & 6 & 1 & 0.79 \\
\hline & $8-5-04.5-0-\mathrm{i}-2.5-3-11.25$ & $\begin{array}{l}\text { A } \\
B\end{array}$ & 1.6 & $4.5 A_{b}$ & $\begin{array}{l}11.00 \\
11.50\end{array}$ & 11.25 & 5500 & 6 & 1 & 0.79 \\
\hline & $8-5-04.5-0-\mathrm{i}-2.5-3-14.25$ & $\begin{array}{l}\text { A } \\
B\end{array}$ & 1.6 & $4.5 A_{b}$ & $\begin{array}{l}14.38 \\
13.88\end{array}$ & 14.13 & 5500 & 6 & 1 & 0.79 \\
\hline & 8-5-S6.5-2\#3-i-2.5-3-9.25 & $\begin{array}{l}\text { A } \\
\text { B }\end{array}$ & 1.8 & $6.5 A_{b}$ & $\begin{array}{l}9.25 \\
9.00\end{array}$ & 9.13 & 5750 & 7 & 1 & 0.79 \\
\hline & 8-5-S6.5-2\#3-i-2.5-3-12.25 & $\begin{array}{l}\text { A } \\
B\end{array}$ & 1.8 & $6.5 A_{b}$ & $\begin{array}{l}12.50 \\
12.13\end{array}$ & 12.31 & 5750 & 7 & 1 & 0.79 \\
\hline & $8-5-04.5-2 \# 3-\mathrm{i}-2.5-3-9.25$ & $\begin{array}{l}\text { A } \\
\text { B }\end{array}$ & 1.6 & $4.5 A_{b}$ & $\begin{array}{l}9.38 \\
9.38\end{array}$ & 9.38 & 5750 & 7 & 1 & 0.79 \\
\hline & 8-5-O4.5-2\#3-i-2.5-3-12.25 & $\begin{array}{l}\text { A } \\
B\end{array}$ & 1.6 & $4.5 A_{b}$ & $\begin{array}{l}12.00 \\
12.00\end{array}$ & 12.00 & 5750 & 7 & 1 & 0.79 \\
\hline & 8-5-S6.5-5\#3-i-2.5-3-8.25 & $\begin{array}{l}\text { A } \\
\text { B }\end{array}$ & 1.8 & $6.5 A_{b}$ & $\begin{array}{l}8.38 \\
8.25\end{array}$ & 8.31 & 5900 & 8 & 1 & 0.79 \\
\hline & 8-5-S6.5-5\#3-i-2.5-3-11.25 & $\begin{array}{l}\text { A } \\
B\end{array}$ & 1.8 & $6.5 A_{b}$ & $\begin{array}{l}10.88 \\
11.00\end{array}$ & 10.94 & 5900 & 8 & 1 & 0.79 \\
\hline & $8-5-04.5-5 \# 3-i-2.5-3-8.25$ & $\begin{array}{l}\text { A } \\
B\end{array}$ & 1.6 & $4.5 A_{b}$ & $\begin{array}{l}8.13 \\
7.88\end{array}$ & 8.00 & 5900 & 8 & 1 & 0.79 \\
\hline & 8-5-O4.5-5\#3-i-2.5-3-11.25 & $\begin{array}{l}A \\
B\end{array}$ & 1.6 & $4.5 A_{b}$ & $\begin{array}{l}11.38 \\
10.88\end{array}$ & 11.13 & 5900 & 8 & 1 & 0.79 \\
\hline \multirow{5}{*}{ Group 7} & 8-5-T9.5-0-i-2.5-3-14.5 & $\begin{array}{l}\text { A } \\
\text { B }\end{array}$ & 1.4 & $9.5 A_{b}$ & $\begin{array}{l}14.25 \\
14.50\end{array}$ & 14.38 & 4970 & 8 & 1 & 0.79 \\
\hline & 8-5-09.1-0-i-2.5-3-14.5 & $\begin{array}{l}\text { A } \\
B\end{array}$ & 1.3 & $9.1 A_{b}$ & $\begin{array}{l}14.38 \\
14.38\end{array}$ & 14.38 & 4970 & 8 & 1 & 0.79 \\
\hline & 8-5-T9.5-5\#3-i-2.5-3-14.5 & $\begin{array}{l}\text { A } \\
\text { B }\end{array}$ & 1.4 & $9.5 A_{b}$ & $\begin{array}{l}14.50 \\
14.25\end{array}$ & 14.38 & 5420 & 13 & 1 & 0.79 \\
\hline & 8-5-09.1-5\#3-i-2.5-3-14.5 & $\begin{array}{l}\mathrm{A} \\
\mathrm{B}\end{array}$ & 1.3 & $9.1 A_{b}$ & $\begin{array}{l}14.06 \\
14.13 \\
\end{array}$ & 14.09 & 4970 & 8 & 1 & 0.79 \\
\hline & (3@5.5)8-5-T9.5-0-i-2.5-3-14.5 & $\begin{array}{l}\text { A } \\
\text { B } \\
\text { C }\end{array}$ & 1.4 & $9.5 A_{b}$ & $\begin{array}{l}14.25 \\
14.25 \\
14.25\end{array}$ & 14.25 & 4960 & 9 & 1 & 0.79 \\
\hline
\end{tabular}


Table A.3 Cont. Comprehensive test results and data for beam-column joint specimens

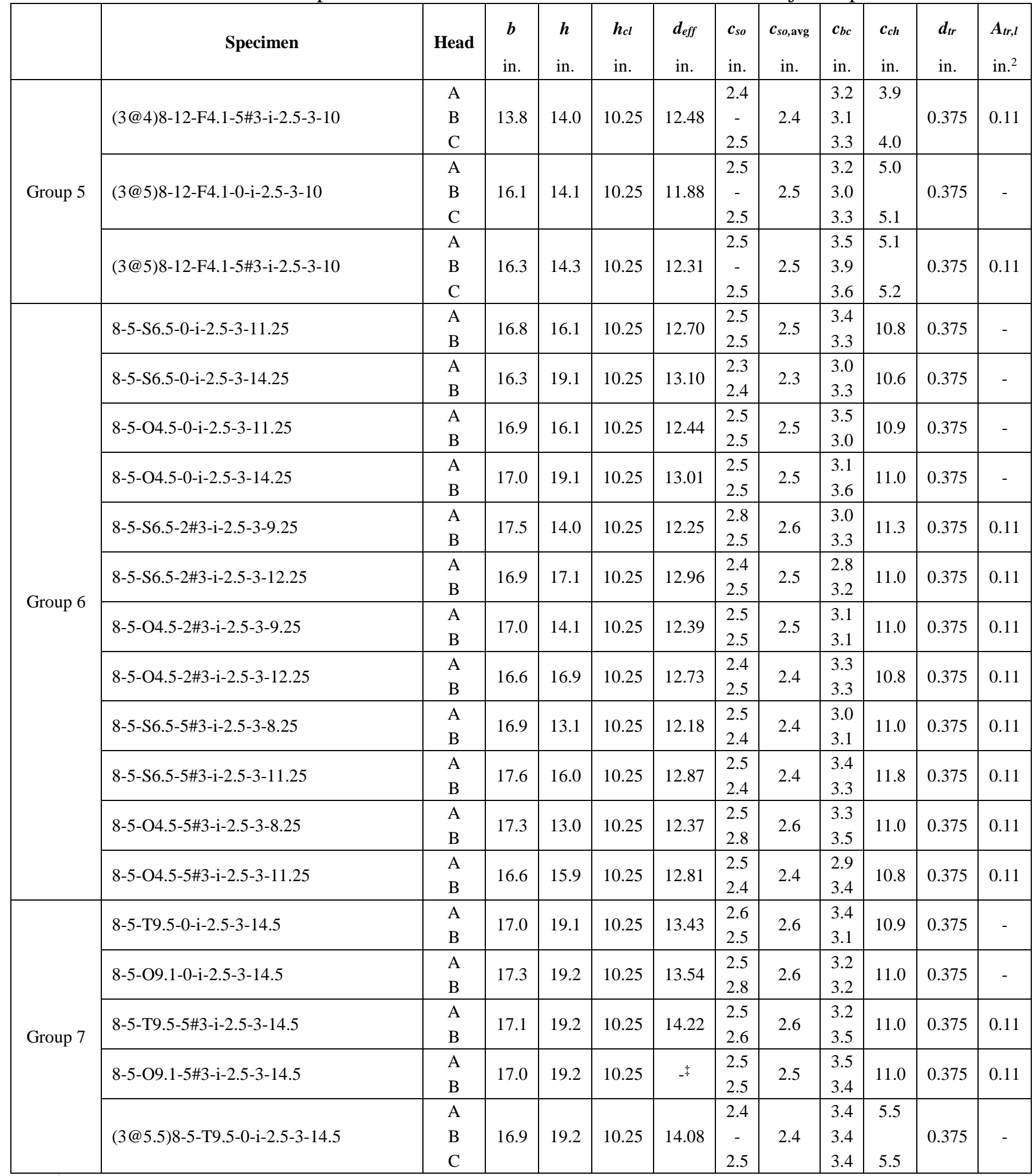

${ }^{\ddagger} d_{\text {eff }}$ was not calculated for specimen with bar yielding 
Table A.3 Cont. Comprehensive test results and data for beam-column joint specimens

\begin{tabular}{|c|c|c|c|c|c|c|c|c|c|c|c|}
\hline & Specimen & Head & $N$ & $\begin{array}{l}\boldsymbol{s t r}^{*} \\
\text { in. }\end{array}$ & $\begin{array}{l}\boldsymbol{A}_{t t} \\
\text { in. }^{2}\end{array}$ & $\begin{array}{l}\boldsymbol{d}_{\text {tro }} \\
\text { in. }\end{array}$ & $\begin{array}{l}\text { Stro }^{*} \\
\text { in. }\end{array}$ & $\begin{array}{l}A_{a b} \\
\text { in. }^{2}\end{array}$ & $n$ & $\begin{array}{l}A_{h s} \\
\text { in. }^{2}\end{array}$ & $\begin{array}{c}\text { Long. } \\
\text { Reinf. } \\
\text { Layout }\end{array}$ \\
\hline \multirow{3}{*}{ Group 5} & (3@4)8-12-F4.1-5\#3-i-2.5-3-10 & $\begin{array}{l}\text { A } \\
\text { B } \\
\text { C }\end{array}$ & 10 & $\begin{array}{c}3 \\
(1.5)\end{array}$ & 0.66 & 0.375 & $\begin{array}{c}3 \\
(1.5)\end{array}$ & 0.66 & 3 & 2.37 & A5 \\
\hline & (3@5)8-12-F4.1-0-i-2.5-3-10 & $\begin{array}{l}\text { A } \\
\text { B } \\
\text { C }\end{array}$ & - & - & - & 0.375 & $\begin{array}{c}3 \\
(1.5)\end{array}$ & 0.66 & 3 & 2.37 & A5 \\
\hline & (3@5)8-12-F4.1-5\#3-i-2.5-3-10 & $\begin{array}{l}A \\
B \\
C\end{array}$ & 10 & $\begin{array}{c}3 \\
(1.5)\end{array}$ & 0.66 & 0.375 & $\begin{array}{c}3 \\
(1.5)\end{array}$ & 0.66 & 3 & 2.37 & A5 \\
\hline \multirow{12}{*}{ Group 6} & 8-5-S6.5-0-i-2.5-3-11.25 & $\begin{array}{l}\text { A } \\
B\end{array}$ & - & - & - & 0.5 & $\begin{array}{c}3.5 \\
(1.75)\end{array}$ & 0.80 & 2 & 1.58 & A4 \\
\hline & 8-5-S6.5-0-i-2.5-3-14.25 & $\begin{array}{l}\text { A } \\
B\end{array}$ & - & - & - & 0.5 & $\begin{array}{c}3.5 \\
(1.75)\end{array}$ & 0.80 & 2 & 1.58 & A4 \\
\hline & $8-5-04.5-0-i-2.5-3-11.25$ & $\begin{array}{l}\text { A } \\
B\end{array}$ & - & - & - & 0.5 & $\begin{array}{c}3.5 \\
(1.75)\end{array}$ & 0.80 & 2 & 1.58 & A4 \\
\hline & $8-5-04.5-0-\mathrm{i}-2.5-3-14.25$ & $\begin{array}{l}\text { A } \\
\text { B }\end{array}$ & - & - & - & 0.5 & $\begin{array}{c}3.5 \\
(1.75)\end{array}$ & 0.80 & 2 & 1.58 & A4 \\
\hline & 8-5-S6.5-2\#3-i-2.5-3-9.25 & $\begin{array}{l}\text { A } \\
B\end{array}$ & 4 & $\begin{array}{l}5.5 \\
(5)\end{array}$ & 0.22 & 0.5 & $\begin{array}{c}3 \\
(1.5)\end{array}$ & 1.20 & 2 & 1.58 & A4 \\
\hline & 8-5-S6.5-2\#3-i-2.5-3-12.25 & $\begin{array}{l}A \\
B\end{array}$ & 4 & $\begin{array}{l}5.5 \\
(5)\end{array}$ & 0.22 & 0.5 & $\begin{array}{c}3 \\
(1.5)\end{array}$ & 1.20 & 2 & 1.58 & A4 \\
\hline & 8-5-O4.5-2\#3-i-2.5-3-9.25 & $\begin{array}{l}\text { A } \\
\text { B }\end{array}$ & 4 & $\begin{array}{l}5.5 \\
(5)\end{array}$ & 0.22 & 0.5 & $\begin{array}{c}3 \\
(1.5)\end{array}$ & 1.20 & 2 & 1.58 & A4 \\
\hline & 8-5-O4.5-2\#3-i-2.5-3-12.25 & $\begin{array}{l}\text { A } \\
B\end{array}$ & 4 & $\begin{array}{l}5.5 \\
(5)\end{array}$ & 0.22 & 0.5 & $\begin{array}{c}3 \\
(1.5)\end{array}$ & 1.20 & 2 & 1.58 & A4 \\
\hline & 8-5-S6.5-5\#3-i-2.5-3-8.25 & $\begin{array}{l}\text { A } \\
\text { B }\end{array}$ & 10 & $\begin{array}{c}3 \\
(1.5)\end{array}$ & 0.66 & 0.5 & $\begin{array}{c}2.5 \\
(1.25)\end{array}$ & 1.20 & 2 & 1.58 & A4 \\
\hline & 8-5-S6.5-5\#3-i-2.5-3-11.25 & $\begin{array}{l}\mathrm{A} \\
\mathrm{B}\end{array}$ & 10 & $\begin{array}{c}3 \\
(1.5) \\
\end{array}$ & 0.66 & 0.5 & $\begin{array}{c}3 \\
(1.5)\end{array}$ & 1.20 & 2 & 1.58 & A4 \\
\hline & 8-5-O4.5-5\#3-i-2.5-3-8.25 & $\begin{array}{l}A \\
B\end{array}$ & 10 & $\begin{array}{c}3 \\
(1.5) \\
\end{array}$ & 0.66 & 0.5 & $\begin{array}{c}2.5 \\
(1.25)\end{array}$ & 1.20 & 2 & 1.58 & A4 \\
\hline & $8-5-04.5-5 \# 3-\mathrm{i}-2.5-3-11.25$ & $\begin{array}{l}\text { A } \\
\text { B }\end{array}$ & 10 & $\begin{array}{c}3 \\
(1.5)\end{array}$ & 0.66 & 0.5 & $\begin{array}{c}3 \\
(1.5)\end{array}$ & 1.20 & 2 & 1.58 & A4 \\
\hline \multirow{5}{*}{ Group 7} & 8-5-T9.5-0-i-2.5-3-14.5 & $\begin{array}{l}\text { A } \\
B\end{array}$ & - & - & - & 0.375 & $\begin{array}{c}4 \\
(2)\end{array}$ & 0.44 & 2 & 1.58 & A4 \\
\hline & 8-5-09.1-0-i-2.5-3-14.5 & $\begin{array}{l}\text { A } \\
B\end{array}$ & - & - & - & 0.375 & $\begin{array}{c}4 \\
(2)\end{array}$ & 0.44 & 2 & 1.58 & A4 \\
\hline & 8-5-T9.5-5\#3-i-2.5-3-14.5 & $\begin{array}{l}\text { A } \\
\text { B }\end{array}$ & 10 & $\begin{array}{c}3 \\
(1.5)\end{array}$ & 0.66 & 0.375 & $\begin{array}{c}4 \\
(2)\end{array}$ & 0.44 & 2 & 1.58 & A6 \\
\hline & 8-5-09.1-5\#3-i-2.5-3-14.5 & $\begin{array}{l}\text { A } \\
B\end{array}$ & 10 & $\begin{array}{c}3 \\
(1.5)\end{array}$ & 0.66 & 0.375 & $\begin{array}{c}4 \\
(2)\end{array}$ & 0.28 & 2 & 1.58 & A6 \\
\hline & (3@5.5)8-5-T9.5-0-i-2.5-3-14.5 & $\begin{array}{l}\text { A } \\
\text { B } \\
\text { C }\end{array}$ & - & - & - & 0.375 & $\begin{array}{c}4 \\
(2)\end{array}$ & 0.44 & 3 & 2.37 & A5 \\
\hline
\end{tabular}

\footnotetext{
${ }^{*}$ Value in parenthesis is the spacing between the first hoop and the center of the headed bar
} 
Table A.3 Cont. Comprehensive test results and data for beam-column joint specimens

\begin{tabular}{|c|c|c|c|c|c|c|c|c|c|c|}
\hline & Specimen & Head & $\begin{array}{c}\text { Failure } \\
\text { Type }\end{array}$ & $\begin{array}{c}\text { Lead (Head) } \\
\text { Slip } \\
\text { in. }\end{array}$ & $\begin{array}{l}\boldsymbol{T}_{\max } \\
\text { kips }\end{array}$ & $\begin{array}{c}\boldsymbol{f}_{\text {su,max }} \\
\mathrm{ksi}\end{array}$ & $\begin{array}{l}\boldsymbol{T}_{\text {ind }} \\
\text { kips }\end{array}$ & $\begin{array}{l}\boldsymbol{T}_{\text {total }} \\
\text { kips }\end{array}$ & $\begin{array}{c}\boldsymbol{T} \\
\text { kips }\end{array}$ & $\begin{array}{l}f_{s u} \\
\mathrm{ksi}\end{array}$ \\
\hline \multirow{9}{*}{ Group 5} & \multirow{3}{*}{ (3@4)8-12-F4.1-5\#3-i-2.5-3-10 } & A & \multirow{3}{*}{$\mathrm{CB} / \mathrm{FP}$} & 0.138 & 64.0 & 81.0 & 64.0 & \multirow{3}{*}{197.1} & \multirow{3}{*}{65.7} & \multirow{3}{*}{83.2} \\
\hline & & $\mathrm{B}$ & & 0.240 & 66.5 & 84.2 & 66.5 & & & \\
\hline & & $\mathrm{C}$ & & 0.260 & 66.7 & 84.4 & 66.6 & & & \\
\hline & \multirow{3}{*}{ (3@5)8-12-F4.1-0-i-2.5-3-10 } & A & \multirow{3}{*}{$\mathrm{CB}$} & 0.079 & 57.1 & 72.3 & 57.1 & \multirow{3}{*}{165.4} & \multirow{3}{*}{55.1} & \multirow{3}{*}{69.7} \\
\hline & & $\mathrm{B}$ & & 0.177 & 55.3 & 70.0 & 55.3 & & & \\
\hline & & $\mathrm{C}$ & & $0.249(0.081)$ & 53.0 & 67.1 & 53.0 & & & \\
\hline & \multirow{3}{*}{ (3@5)8-12-F4.1-5\#3-i-2.5-3-10 } & $\mathrm{A}$ & \multirow{3}{*}{$\mathrm{CB} / \mathrm{FP}$} & 0.164 & 77.2 & 97.7 & 77.2 & \multirow{3}{*}{209.1} & \multirow{3}{*}{69.7} & \multirow{3}{*}{88.2} \\
\hline & & B & & 0.123 & 65.4 & 82.8 & 65.4 & & & \\
\hline & & $\mathrm{C}$ & & 0.122 & 66.7 & 84.4 & 66.6 & & & \\
\hline \multirow{24}{*}{ Group 6} & \multirow{2}{*}{ 8-5-S6.5-0-i-2.5-3-11.25 } & A & \multirow{2}{*}{$\mathrm{SB} / \mathrm{FP}$} & 0.161 & 74.9 & 94.8 & 74.9 & \multirow{2}{*}{151.1} & \multirow{2}{*}{75.6} & 95.6 \\
\hline & & $\mathrm{B}$ & & - & 76.2 & 96.5 & 76.2 & & & \\
\hline & $8-5-S 6$ 5-0-i-2 5-3-14 25 & A & SB/FP & $(0.054)$ & 87.5 & 110.8 & 87.5 & 1754 & 877 & 1110 \\
\hline & $0-3-30.5-0-1-2.5-3-14.25$ & $\mathrm{~B}$ & $\mathrm{SD} / \mathrm{FP}$ & - & 103.4 & 130.9 & 88.0 & $1 / 3.4$ & $0 \% .7$ & 111.0 \\
\hline & & A & SB/FP & 0.037 & 67.6 & 85.6 & 67.6 & 1348 & & \\
\hline & $8-5-04.5-0-1-2.5-3-11.25$ & B & $\mathrm{SB} / \mathrm{HP}$ & 0.198 & 67.2 & 85.0 & 67.2 & 134.8 & $6 / .4$ & 85.3 \\
\hline & $8-5-045_{-0}{ }_{-i-2} 5_{-3}-1425$ & A & SB/EP & $0.214(0.023)$ & 103.5 & 131.0 & 84.2 & 1700 & 850 & 1076 \\
\hline & $0-5-04.5-0-1-2.5-3-14.25$ & $\mathrm{~B}$ & SB/FP & 0.113 & 85.8 & 108.6 & 85.8 & $1 / 0.0$ & 85.0 & $10 / .0$ \\
\hline & 8-5-S6 5-2\#3-i-25-3-925 & A & $C \mathrm{~B}$ & $(0.012)$ & 62.6 & 79.2 & 62.6 & 1267 & 624 & 802 \\
\hline & $0-2-30.3-2+43-1-2.3-3-2.53$ & $\mathrm{~B}$ & CD & - & 64.1 & 81.2 & 64.1 & 120.1 & 03.4 & 00.2 \\
\hline & $8-5-S 6$ 5-2\#3-i-2 5-3-12 25 & A & $\mathrm{SB} / \mathrm{FP}$ & 0.340 & 84.6 & 107.1 & 84.6 & 1719 & 860 & 1088 \\
\hline & & $\mathrm{B}$ & & 0.254 & 89.3 & 113.0 & 87.3 & & 80.0 & \\
\hline & 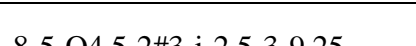 & $\mathrm{A}$ & SD/ED & 0.309 & 67.6 & 85.6 & 67.1 & 1250 & 670 & 960 \\
\hline & $8-5-04.5-2 \# 3-1-2.5-3-9.25$ & B & $\mathrm{SB} / \mathrm{FP}$ & 0.205 & 68.7 & 86.9 & 68.7 & 135.8 & $6 / .9$ & 86.0 \\
\hline & $050452+3 \div 252125$ & $\mathrm{~A}$ & SD/ED & 0.305 & 82.8 & 104.8 & 77.4 & 1570 & 705 & 004 \\
\hline & $8-5-04.5-\angle A 3-1-2.3-3-12.25$ & $\mathrm{~B}$ & SB/FP & 0.220 & 79.6 & 100.8 & 79.6 & $15 \% .0$ & 18.5 & 99.4 \\
\hline & $8-5-S 6$ 5-5\#3-i-2 5-3-825 & A & $C \mathrm{~B} / \mathrm{FP}$ & 0.363 & 61.9 & 78.4 & 61.9 & 1241 & 620 & 785 \\
\hline & $8-5-20.5-2+3-1-2.5-3-8.25$ & $\mathrm{~B}$ & CD/FP & 0.500 & 62.2 & 78.7 & 62.2 & 124.1 & 02.0 & 70.5 \\
\hline & Q 5 $S 65-5 \# 2-25-31125$ & A & SP/ED & - & 100.8 & 127.6 & 84.2 & 1600 & 845 & \\
\hline & $8-5-56.5-5 \# 3-1-2.5-3-11.25$ & B & $\mathrm{SB} / \mathrm{FP}$ & 0.046 & 84.7 & 107.2 & 84.7 & 169.0 & 84.5 & 106.9 \\
\hline & $8-5-O 4$ 5-5\#3-i-2 5-3-8 25 & A & SB/FP & 0.457 & 68.3 & 86.5 & 68.3 & 1368 & & \\
\hline & $8-5-04.5-5 \# 3-1-2.5-3-8.25$ & $\mathrm{~B}$ & SB/FP & 0.383 & 68.5 & 86.7 & 68.5 & 136.8 & 68.4 & 86.6 \\
\hline & 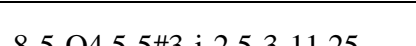 & $\mathrm{A}$ & SP/FD & 0.171 & 85.0 & 107.6 & 82.1 & 1615 & 827 & 1041 \\
\hline & $0-2-04.5-2+3-1-2.3-2-11.53$ & $\mathrm{~B}$ & $\mathrm{SD} / \mathrm{FH}$ & - & 82.4 & 104.3 & 82.4 & 164.5 & 82.2 & 104.1 \\
\hline & 8 5-T0 5-0 & A & SP/ED & 0.130 & 91.5 & 115.8 & 91.5 & & & \\
\hline & $8-5-19.5-0-1-2.5-3-14.5$ & B & SB/FP & 0.312 & 115.9 & 146.7 & 91.8 & 183.3 & 91.7 & 116.0 \\
\hline & & $\mathrm{A}$ & SP/ED & 0.060 & 94.6 & 119.7 & 94.6 & 1806 & 048 & 1200 \\
\hline & 8-5-09.1-0-1-2.5-3-14.5 & B & SB/FP & 0.186 & 95.2 & 120.5 & 95.0 & 189.6 & 94.8 & 120.0 \\
\hline & 8 5 T9 5 5\#2 i 252145 & A & SP/ED & - & $120.7^{\ddagger}$ & $152.8^{\ddagger}$ & 120.6 & 2420 & 1210 & 1522 \\
\hline Group 7 & $8-5-19.5-5 \# 3-1-2.5-3-14.5$ & B & $\mathrm{SB} / \mathrm{HP}$ & - & 121.4 & 153.7 & 121.4 & 242.0 & 121.0 & 153.2 \\
\hline & 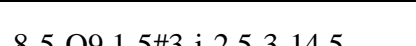 & A & $\mathrm{Y}$ & 0.050 & 118.8 & 150.4 & 118.8 & 2305 & 1102 & 1509 \\
\hline & $0-2-27.1-2+3-1-5.2-3-14.3$ & B & $\mathbf{I}$ & - & 119.7 & 151.5 & 119.7 & 230.5 & 119.5 & 100.5 \\
\hline & & $\mathrm{A}$ & & 0.156 & 68.7 & 87.0 & 68.7 & & & \\
\hline & (3@5.5)8-5-T9.5-0-i-2.5-3-14.5 & $\mathrm{B}$ & CB & 0.138 & 78.8 & 99.7 & 78.8 & 220.2 & 73.4 & 92.9 \\
\hline & & $\mathrm{C}$ & & 0.217 & 72.6 & 91.9 & 72.6 & & & \\
\hline
\end{tabular}

\footnotetext{
${ }^{\ddagger}$ No anchorage failure on the bar
} 
Table A.3 Cont. Comprehensive test results and data for beam-column joint specimens

\begin{tabular}{|c|c|c|c|c|c|c|c|c|c|c|}
\hline & Specimen & Head & $\begin{array}{l}\boldsymbol{c}_{\boldsymbol{o}} \\
\text { in. }\end{array}$ & $A_{b r g}$ & $\begin{array}{l}\ell e h \\
\text { in. }\end{array}$ & $\begin{array}{c}\ell \text { eh, avg } \\
\text { in. }\end{array}$ & $\begin{array}{l}\boldsymbol{f}_{c m} \\
\text { psi }\end{array}$ & $\begin{array}{l}\text { Age } \\
\text { days }\end{array}$ & $\begin{array}{l}\boldsymbol{d}_{b} \\
\text { in. }\end{array}$ & $\begin{array}{l}\boldsymbol{A}_{\boldsymbol{b}} \\
\text { in. }^{2}\end{array}$ \\
\hline \multirow{7}{*}{ Group 7} & (3@5.5)8-5-09.1-0-i-2.5-3-14.5 & $\begin{array}{l}\text { A } \\
\text { B } \\
\text { C }\end{array}$ & 1.3 & $9.1 A_{b}$ & $\begin{array}{l}14.31 \\
14.50 \\
14.25\end{array}$ & 14.35 & 4960 & 9 & 1 & 0.79 \\
\hline & (3@5.5)8-5-T9.5-5\#3-i-2.5-3-14.5 & $\begin{array}{l}\text { A } \\
\text { B } \\
\text { C }\end{array}$ & 1.4 & $9.5 A_{b}$ & $\begin{array}{l}14.50 \\
14.38 \\
14.38 \\
\end{array}$ & 14.42 & 5370 & 10 & 1 & 0.79 \\
\hline & (3@5.5)8-5-09.1-5\#3-i-2.5-3-14.5 & $\begin{array}{l}\text { A } \\
B \\
C \\
\end{array}$ & 1.3 & $9.1 A_{b}$ & $\begin{array}{l}14.06 \\
14.44 \\
14.31 \\
\end{array}$ & 14.27 & 5420 & 13 & 1 & 0.79 \\
\hline & (4@3.7)8-5-T9.5-0-i-2.5-3-14.5 & $\begin{array}{l}\text { A } \\
B \\
C \\
D\end{array}$ & 1.4 & $9.5 A_{b}$ & $\begin{array}{l}14.25 \\
14.38 \\
14.25 \\
14.19\end{array}$ & 14.30 & 5570 & 14 & 1 & 0.79 \\
\hline & (4@3.7)8-5-09.1-0-i-2.5-3-14.5 & $\begin{array}{l}A \\
B \\
C \\
D \\
\end{array}$ & 1.3 & $9.1 A_{b}$ & $\begin{array}{l}14.06 \\
14.06 \\
14.06 \\
14.06\end{array}$ & 14.06 & 5570 & 14 & 1 & 0.79 \\
\hline & (4@3.7)8-5-T9.5-5\#3-i-2.5-3-14.5 & $\begin{array}{l}\text { A } \\
\text { B } \\
\text { C } \\
\text { D } \\
\end{array}$ & 1.4 & $9.5 A_{b}$ & $\begin{array}{l}14.44 \\
14.38 \\
14.63 \\
14.50 \\
\end{array}$ & 14.50 & 5570 & 14 & 1 & 0.79 \\
\hline & (4@3.7)8-5-O9.1-5\#3-i-2.5-3-14.5 & $\begin{array}{l}\text { A } \\
\text { B } \\
\text { C } \\
\text { D }\end{array}$ & 1.3 & $9.1 A_{b}$ & $\begin{array}{l}14.44 \\
14.44 \\
14.63 \\
14.44\end{array}$ & 14.50 & 5570 & 14 & 1 & 0.79 \\
\hline \multirow{9}{*}{ Group 8} & 8-15-T4.0-0-i-2.5-4.5-9.5 & $\begin{array}{l}A \\
B\end{array}$ & 1.9 & $4.0 A_{b}$ & $\begin{array}{l}9.50 \\
9.50\end{array}$ & 9.50 & 16030 & 88 & 1 & 0.79 \\
\hline & 8-15-S9.5-0-i-2.5-3-9.5 & $\begin{array}{l}\text { A } \\
\text { B }\end{array}$ & 1.4 & $9.5 A_{b}$ & $\begin{array}{l}9.50 \\
9.50 \\
\end{array}$ & 9.50 & 16030 & 88 & 1 & 0.79 \\
\hline & 8-15-S14.9-0-i-2.5-3-9.5 & $\begin{array}{l}\mathrm{A} \\
\mathrm{B}\end{array}$ & 1.0 & $14.9 A_{b}$ & $\begin{array}{l}9.63 \\
9.75 \\
\end{array}$ & 9.69 & 16030 & 88 & 1 & 0.79 \\
\hline & 8-15-T4.0-2\#3-i-2.5-4.5-7 & $\begin{array}{l}A \\
B\end{array}$ & 1.9 & $4.0 A_{b}$ & $\begin{array}{l}7.13 \\
7.00 \\
\end{array}$ & 7.06 & 16030 & 87 & 1 & 0.79 \\
\hline & 8-15-S9.5-2\#3-i-2.5-3-7 & $\begin{array}{l}\mathrm{A} \\
\mathrm{B}\end{array}$ & 1.4 & $9.5 A_{b}$ & $\begin{array}{l}7.13 \\
7.00 \\
\end{array}$ & 7.06 & 16030 & 87 & 1 & 0.79 \\
\hline & 8-15-S14.9-2\#3-i-2.5-3-7 & $\begin{array}{l}\text { A } \\
B\end{array}$ & 1.0 & $14.9 A_{b}$ & $\begin{array}{l}7.00 \\
7.00 \\
\end{array}$ & 7.00 & 16030 & 87 & 1 & 0.79 \\
\hline & 8-15-T4.0-5\#3-i-2.5-4.5-5.5 & $\begin{array}{l}\mathrm{A} \\
\mathrm{B} \\
\end{array}$ & 1.9 & $4.0 A_{b}$ & $\begin{array}{l}5.50 \\
5.50 \\
\end{array}$ & 5.50 & 16030 & 88 & 1 & 0.79 \\
\hline & 8-15-S9.5-5\#3-i-2.5-3-5.5 & $\begin{array}{l}\mathrm{A} \\
\mathrm{B}\end{array}$ & 1.4 & $9.5 A_{b}$ & $\begin{array}{l}5.75 \\
5.50 \\
\end{array}$ & 5.63 & 16030 & 88 & 1 & 0.79 \\
\hline & 8-15-S14.9-5\#3-i-2.5-3-5.5 & $\begin{array}{l}\text { A } \\
\text { B }\end{array}$ & 1.0 & $14.9 A_{b}$ & $\begin{array}{l}5.50 \\
5.50 \\
\end{array}$ & 5.50 & 16030 & 88 & 1 & 0.79 \\
\hline
\end{tabular}


Table A.3 Cont. Comprehensive test results and data for beam-column joint specimens

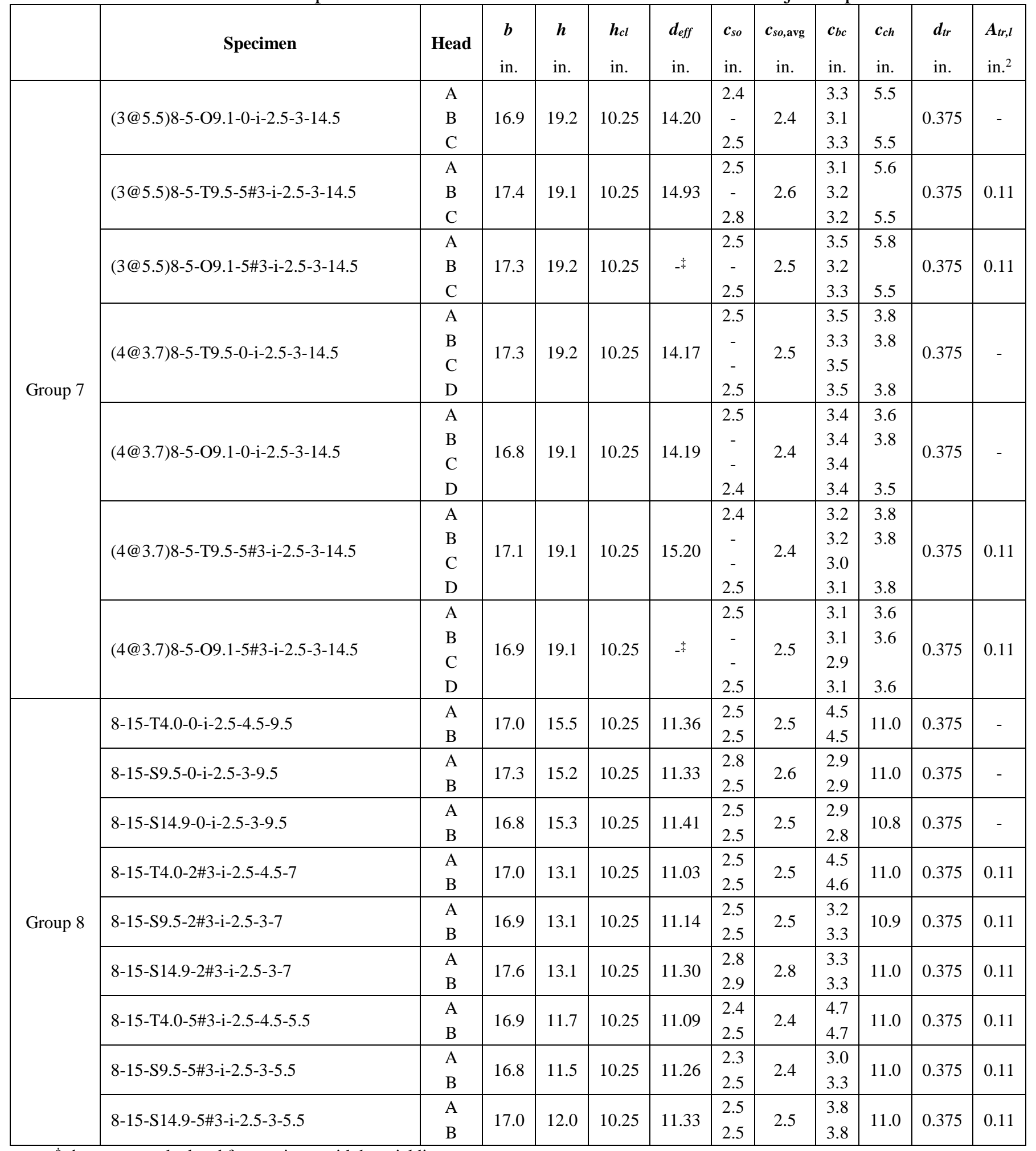

\footnotetext{
${ }^{\ddagger} d_{\text {eff }}$ was not calculated for specimen with bar yielding
} 
Table A.3 Cont. Comprehensive test results and data for beam-column joint specimens

\begin{tabular}{|c|c|c|c|c|c|c|c|c|c|c|c|}
\hline & Specimen & Head & $N$ & $\begin{array}{l}\boldsymbol{s}_{t r}{ }^{*} \\
\text { in. }\end{array}$ & $\begin{array}{l}\boldsymbol{A}_{t t} \\
\text { in. }^{2}\end{array}$ & $\begin{array}{l}\boldsymbol{d}_{\text {tro }} \\
\text { in. }\end{array}$ & $\begin{array}{l}\text { Stro }^{*} \\
\text { in. }\end{array}$ & $\begin{array}{l}\boldsymbol{A}_{\boldsymbol{a} \boldsymbol{b}} \\
\text { in. }^{2}\end{array}$ & $n$ & $\begin{array}{l}A_{\text {hs }} \\
\text { in. }^{2}\end{array}$ & $\begin{array}{l}\text { Long. } \\
\text { Reinf. } \\
\text { Layout }\end{array}$ \\
\hline \multirow{7}{*}{ Group 7} & (3@5.5)8-5-09.1-0-i-2.5-3-14.5 & $\begin{array}{l}\text { A } \\
\text { B } \\
\text { C }\end{array}$ & - & - & - & 0.375 & $\begin{array}{c}4 \\
(2)\end{array}$ & 0.44 & 3 & 2.37 & A5 \\
\hline & (3@5.5)8-5-T9.5-5\#3-i-2.5-3-14.5 & $\begin{array}{l}\text { A } \\
B \\
C\end{array}$ & 10 & $\begin{array}{c}3 \\
(1.5)\end{array}$ & 0.66 & 0.375 & $\begin{array}{c}4 \\
(2)\end{array}$ & 0.44 & 3 & 2.37 & A5 \\
\hline & (3@5.5)8-5-O9.1-5\#3-i-2.5-3-14.5 & $\begin{array}{l}\text { A } \\
B \\
C\end{array}$ & 10 & $\begin{array}{c}3 \\
(1.5)\end{array}$ & 0.66 & 0.375 & $\begin{array}{c}4 \\
(2)\end{array}$ & 0.19 & 3 & 2.37 & A5 \\
\hline & (4@3.7)8-5-T9.5-0-i-2.5-3-14.5 & $\begin{array}{l}\mathrm{A} \\
\mathrm{B} \\
\mathrm{C} \\
\mathrm{D}\end{array}$ & - & - & - & 0.375 & $\begin{array}{c}4 \\
(2)\end{array}$ & 0.44 & 4 & 3.16 & A5 \\
\hline & (4@3.7)8-5-O9.1-0-i-2.5-3-14.5 & $\begin{array}{l}\mathrm{A} \\
\mathrm{B} \\
\mathrm{C} \\
\mathrm{D}\end{array}$ & - & - & - & 0.375 & $\begin{array}{c}4 \\
(2)\end{array}$ & 0.44 & 4 & 3.16 & A5 \\
\hline & (4@3.7)8-5-T9.5-5\#3-i-2.5-3-14.5 & $\begin{array}{l}\text { A } \\
\text { B } \\
\text { C } \\
\text { D }\end{array}$ & 10 & $\begin{array}{c}3 \\
(1.5)\end{array}$ & 0.66 & 0.375 & $\begin{array}{c}3 \\
(1.5)\end{array}$ & 0.66 & 4 & 3.16 & A5 \\
\hline & (4@3.7)8-5-09.1-5\#3-i-2.5-3-14.5 & $\begin{array}{l}\mathrm{A} \\
\mathrm{B} \\
\mathrm{C} \\
\mathrm{D}\end{array}$ & 10 & $\begin{array}{c}3 \\
(1.5)\end{array}$ & 0.66 & 0.375 & $\begin{array}{c}3 \\
(1.5)\end{array}$ & 0.21 & 4 & 3.16 & A5 \\
\hline \multirow{9}{*}{ Group 8} & 8-15-T4.0-0-i-2.5-4.5-9.5 & $\begin{array}{l}\text { A } \\
\text { B }\end{array}$ & - & - & - & 0.5 & $\begin{array}{c}4 \\
(2)\end{array}$ & 0.80 & 2 & 1.58 & A6 \\
\hline & 8-15-S9.5-0-i-2.5-3-9.5 & $\begin{array}{l}A \\
B\end{array}$ & - & - & - & 0.5 & $\begin{array}{c}4 \\
(2) \\
\end{array}$ & 0.80 & 2 & 1.58 & A6 \\
\hline & 8-15-S14.9-0-i-2.5-3-9.5 & $\begin{array}{l}A \\
B\end{array}$ & - & - & - & 0.5 & $\begin{array}{c}4 \\
(2) \\
\end{array}$ & 0.80 & 2 & 1.58 & A6 \\
\hline & 8-15-T4.0-2\#3-i-2.5-4.5-7 & $\begin{array}{l}A \\
B\end{array}$ & 4 & $\begin{array}{l}5.5 \\
(5)\end{array}$ & 0.22 & 0.5 & $\begin{array}{c}4 \\
(2) \\
\end{array}$ & 0.80 & 2 & 1.58 & A6 \\
\hline & 8-15-S9.5-2\#3-i-2.5-3-7 & $\begin{array}{l}\text { A } \\
\text { B }\end{array}$ & 4 & $\begin{array}{l}5.5 \\
(5)\end{array}$ & 0.22 & 0.5 & $\begin{array}{c}4 \\
(2)\end{array}$ & 0.80 & 2 & 1.58 & A6 \\
\hline & 8-15-S14.9-2\#3-i-2.5-3-7 & $\begin{array}{l}\text { A } \\
\text { B }\end{array}$ & 4 & $\begin{array}{l}5.5 \\
(5)\end{array}$ & 0.22 & 0.5 & $\begin{array}{c}4 \\
(2)\end{array}$ & 0.80 & 2 & 1.58 & A6 \\
\hline & 8-15-T4.0-5\#3-i-2.5-4.5-5.5 & $\begin{array}{l}\mathrm{A} \\
\mathrm{B}\end{array}$ & 10 & $\begin{array}{c}3 \\
(1.5) \\
\end{array}$ & 0.66 & 0.5 & $\begin{array}{c}4 \\
(2) \\
\end{array}$ & 0.80 & 2 & 1.58 & A7 \\
\hline & 8-15-S9.5-5\#3-i-2.5-3-5.5 & $\begin{array}{l}\text { A } \\
\text { B }\end{array}$ & 10 & $\begin{array}{c}3 \\
(1.5)\end{array}$ & 0.66 & 0.5 & $\begin{array}{c}4 \\
(2)\end{array}$ & 0.80 & 2 & 1.58 & A7 \\
\hline & 8-15-S14.9-5\#3-i-2.5-3-5.5 & $\begin{array}{l}\text { A } \\
\text { B }\end{array}$ & 10 & $\begin{array}{c}3 \\
(1.5)\end{array}$ & 0.66 & 0.5 & $\begin{array}{c}4 \\
(2)\end{array}$ & 0.80 & 2 & 1.58 & A7 \\
\hline
\end{tabular}

${ }^{*}$ Value in parenthesis is the spacing between the first hoop and the center of the headed bar 
Table A.3 Cont. Comprehensive test results and data for beam-column joint specimens

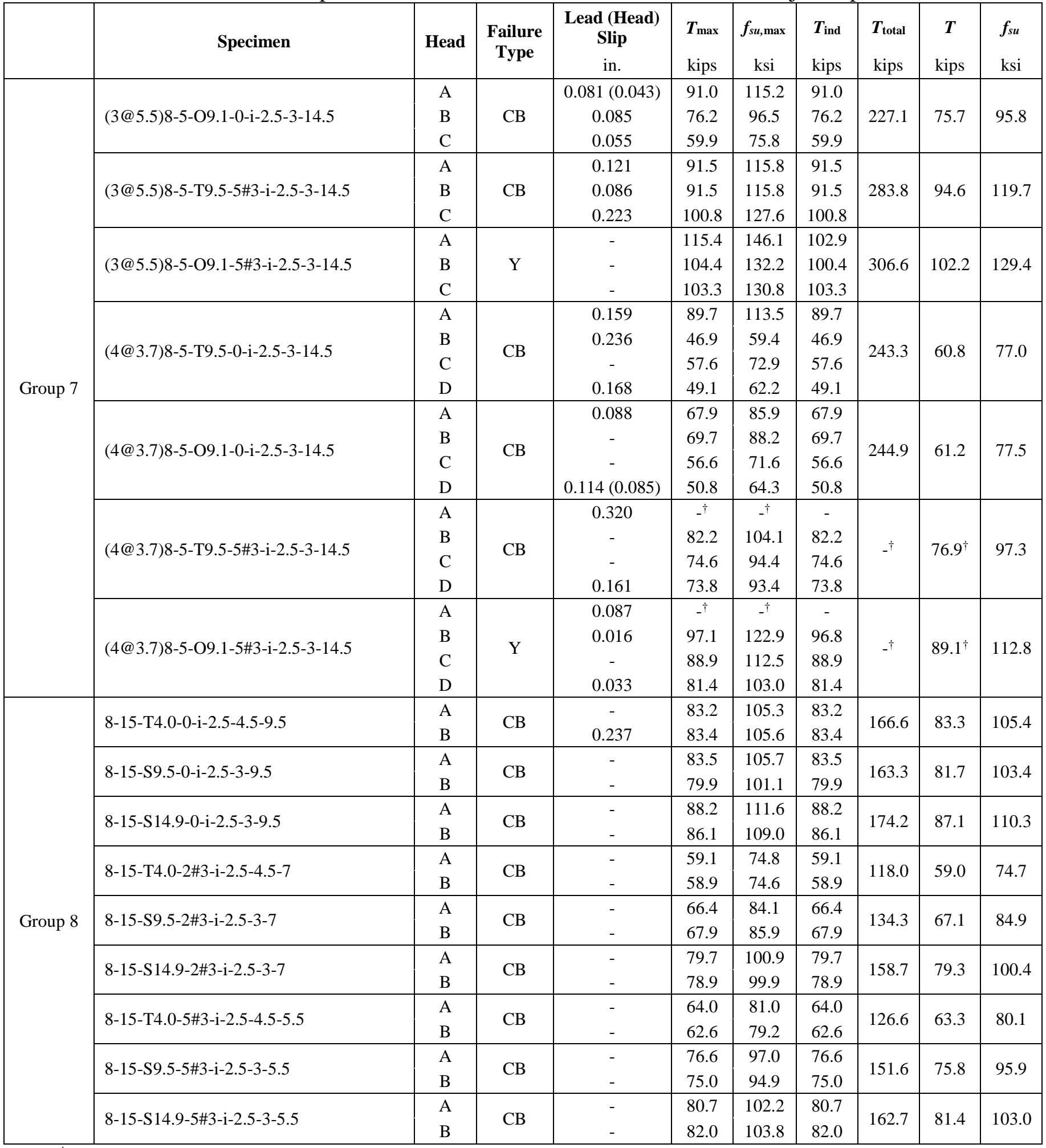

\footnotetext{
${ }^{\dagger}$ Load on headed bar A was not recorded due to a malfunction of load cell; $T$ taken as the average load of the other three bars.
} 
Table A.3 Cont. Comprehensive test results and data for beam-column joint specimens

\begin{tabular}{|c|c|c|c|c|c|c|c|c|c|c|}
\hline & Specimen & Head & $\begin{array}{l}c_{o} \\
\text { in. }\end{array}$ & $A_{b r g}$ & $\begin{array}{l}\ell e h \\
\text { in. }\end{array}$ & $\begin{array}{c}\ell \text { eh,avg } \\
\text { in. }\end{array}$ & $\begin{array}{l}f_{c m} \\
\text { psi }\end{array}$ & $\begin{array}{l}\text { Age } \\
\text { days }\end{array}$ & $\begin{array}{l}\boldsymbol{d}_{b} \\
\text { in. }\end{array}$ & $\begin{array}{l}\boldsymbol{A}_{\boldsymbol{b}} \\
\text { in. }^{2}\end{array}$ \\
\hline \multirow{15}{*}{ Group 9} & 8-8-T9.5-0-i-2.5-3-9.5 & $\begin{array}{l}\text { A } \\
B\end{array}$ & 1.4 & $9.5 A_{b}$ & $\begin{array}{l}9.50 \\
9.25 \\
\end{array}$ & 9.38 & 9040 & 12 & 1 & 0.79 \\
\hline & 8-8-T9.5-2\#3-i-2.5-3-9.5 & $\begin{array}{l}\mathrm{A} \\
\mathrm{B}\end{array}$ & 1.4 & $9.5 A_{b}$ & $\begin{array}{l}9.00 \\
9.38\end{array}$ & 9.19 & 9040 & 12 & 1 & 0.79 \\
\hline & (3@4)8-8-T9.5-0-i-2.5-3-9.5 & $\begin{array}{l}\mathrm{A} \\
\mathrm{B} \\
\mathrm{C}\end{array}$ & 1.4 & $9.5 A_{b}$ & $\begin{array}{l}9.00 \\
9.50 \\
9.25 \\
\end{array}$ & 9.25 & 9040 & 12 & 1 & 0.79 \\
\hline & (3@4)8-8-T9.5-2\#3-i-2.5-3-9.5 & $\begin{array}{l}\text { A } \\
\text { B } \\
\text { C }\end{array}$ & 1.4 & $9.5 A_{b}$ & $\begin{array}{l}9.75 \\
9.50 \\
9.50 \\
\end{array}$ & 9.58 & 9040 & 12 & 1 & 0.79 \\
\hline & (3@5)8-8-T9.5-0-i-2.5-3-9.5 & $\begin{array}{l}\text { A } \\
B \\
C\end{array}$ & 1.4 & $9.5 A_{b}$ & $\begin{array}{l}9.50 \\
9.75 \\
9.25 \\
\end{array}$ & 9.50 & 9940 & 11 & 1 & 0.79 \\
\hline & (3@5)8-8-T9.5-2\#3-i-2.5-3-9.5 & $\begin{array}{l}\text { A } \\
\text { B } \\
\text { C }\end{array}$ & 1.4 & $9.5 A_{b}$ & $\begin{array}{l}9.50 \\
9.50 \\
9.25 \\
\end{array}$ & 9.42 & 9940 & 11 & 1 & 0.79 \\
\hline & (3@7)8-8-T9.5-0-i-2.5-3-9.5 & $\begin{array}{l}\text { A } \\
B \\
C\end{array}$ & 1.4 & $9.5 A_{b}$ & $\begin{array}{l}9.50 \\
9.63 \\
9.38\end{array}$ & 9.50 & 10180 & 10 & 1 & 0.79 \\
\hline & (3@7)8-8-T9.5-2\#3-i-2.5-3-9.5 & $\begin{array}{l}\text { A } \\
\text { B } \\
\text { C }\end{array}$ & 1.4 & $9.5 A_{b}$ & $\begin{array}{l}9.50 \\
9.75 \\
9.50\end{array}$ & 9.58 & 10180 & 10 & 1 & 0.79 \\
\hline & 8-8-T9.5-0-i-2.5-3-14.5 & $\begin{array}{l}\mathrm{A} \\
\mathrm{B}\end{array}$ & 1.4 & $9.5 A_{b}$ & $\begin{array}{l}14.50 \\
14.25\end{array}$ & 14.38 & 10180 & 10 & 1 & 0.79 \\
\hline & (3@4)8-8-T9.5-0-i-2.5-3-14.5 & $\begin{array}{l}\mathrm{A} \\
\mathrm{B} \\
\mathrm{C} \\
\end{array}$ & 1.4 & $9.5 A_{b}$ & $\begin{array}{l}14.25 \\
14.75 \\
14.75 \\
\end{array}$ & 14.58 & 9040 & 12 & 1 & 0.79 \\
\hline & (3@4)8-8-T9.5-2\#3-i-2.5-3-14.5 & $\begin{array}{l}\mathrm{A} \\
\mathrm{B} \\
\mathrm{C}\end{array}$ & 1.4 & $9.5 A_{b}$ & $\begin{array}{l}14.50 \\
14.50 \\
14.25 \\
\end{array}$ & 14.42 & 9040 & 12 & 1 & 0.79 \\
\hline & (3@5)8-8-T9.5-0-i-2.5-3-14.5 & $\begin{array}{l}\text { A } \\
B \\
C\end{array}$ & 1.4 & $9.5 A_{b}$ & $\begin{array}{l}14.75 \\
14.50 \\
14.50\end{array}$ & 14.58 & 9940 & 11 & 1 & 0.79 \\
\hline & (3@5)8-8-T9.5-2\#3-i-2.5-3-14.5 & $\begin{array}{l}\text { A } \\
B \\
C\end{array}$ & 1.4 & $9.5 A_{b}$ & $\begin{array}{l}14.00 \\
14.25 \\
14.00\end{array}$ & 14.08 & 9940 & 11 & 1 & 0.79 \\
\hline & (3@7)8-8-T9.5-0-i-2.5-3-14.5 & $\begin{array}{l}\text { A } \\
B \\
C\end{array}$ & 1.4 & $9.5 A_{b}$ & $\begin{array}{l}14.44 \\
14.56 \\
14.63\end{array}$ & 14.54 & 10180 & 10 & 1 & 0.79 \\
\hline & (3@7)8-8-T9.5-2\#3-i-2.5-3-14.5 & $\begin{array}{l}\text { A } \\
B \\
C\end{array}$ & 1.4 & $9.5 A_{b}$ & $\begin{array}{l}14.50 \\
14.63 \\
14.50\end{array}$ & 14.54 & 10180 & 10 & 1 & 0.79 \\
\hline
\end{tabular}


Table A.3 Cont. Comprehensive test results and data for beam-column joint specimens

\begin{tabular}{|c|c|c|c|c|c|c|c|c|c|c|c|c|}
\hline & Specimen & Head & $\begin{array}{c}\boldsymbol{b} \\
\text { in. }\end{array}$ & $\begin{array}{c}\boldsymbol{h} \\
\text { in. }\end{array}$ & $\begin{array}{l}\boldsymbol{h}_{\boldsymbol{c l}} \\
\text { in. }\end{array}$ & $\begin{array}{l}\boldsymbol{d}_{\text {eff }} \\
\text { in. }\end{array}$ & $\begin{array}{l}c_{s o} \\
\text { in. }\end{array}$ & $\begin{array}{c}\boldsymbol{C}_{\boldsymbol{s o}, \text { avg }} \\
\text { in. }\end{array}$ & $\begin{array}{l}c_{b c} \\
\text { in. }\end{array}$ & $\begin{array}{l}\boldsymbol{c}_{\boldsymbol{c h}} \\
\text { in. }\end{array}$ & $\begin{array}{l}\boldsymbol{d}_{t r} \\
\text { in. }\end{array}$ & $\begin{array}{l}A_{t r, l} \\
\text { in. }^{2}\end{array}$ \\
\hline \multirow{15}{*}{ Group 9} & 8-8-T9.5-0-i-2.5-3-9.5 & $\begin{array}{l}\text { A } \\
B\end{array}$ & 17.0 & 14.3 & 10.25 & 11.79 & $\begin{array}{l}2.5 \\
2.5\end{array}$ & 2.5 & $\begin{array}{l}3.3 \\
3.5\end{array}$ & 11.0 & 0.375 & - \\
\hline & 8-8-T9.5-2\#3-i-2.5-3-9.5 & $\begin{array}{l}\text { A } \\
B\end{array}$ & 17.0 & 14.0 & 10.25 & 11.87 & $\begin{array}{l}2.8 \\
2.5\end{array}$ & 2.6 & $\begin{array}{l}3.5 \\
3.1\end{array}$ & 10.8 & 0.375 & 0.11 \\
\hline & (3@4)8-8-T9.5-0-i-2.5-3-9.5 & $\begin{array}{l}\text { A } \\
\text { B } \\
\text { C }\end{array}$ & 14.0 & 14.0 & 10.25 & 11.98 & $\begin{array}{c}2.5 \\
- \\
2.5\end{array}$ & 2.5 & $\begin{array}{l}3.5 \\
3.0 \\
3.3\end{array}$ & $\begin{array}{l}4.0 \\
4.0\end{array}$ & 0.375 & - \\
\hline & (3@4)8-8-T9.5-2\#3-i-2.5-3-9.5 & $\begin{array}{l}\text { A } \\
B \\
C\end{array}$ & 14.0 & 14.3 & 10.25 & 12.47 & $\begin{array}{c}2.5 \\
- \\
2.5\end{array}$ & 2.5 & $\begin{array}{l}3.0 \\
3.3 \\
3.3\end{array}$ & $\begin{array}{l}4.0 \\
4.0\end{array}$ & 0.375 & 0.11 \\
\hline & (3@5)8-8-T9.5-0-i-2.5-3-9.5 & $\begin{array}{l}\text { A } \\
B \\
C \\
\end{array}$ & 16.0 & 14.3 & 10.25 & 11.77 & $\begin{array}{c}2.5 \\
- \\
2.5 \\
\end{array}$ & 2.5 & $\begin{array}{l}3.3 \\
3.0 \\
3.5 \\
\end{array}$ & $\begin{array}{l}5.0 \\
5.0 \\
\end{array}$ & 0.375 & - \\
\hline & (3@5)8-8-T9.5-2\#3-i-2.5-3-9.5 & $\begin{array}{l}\text { A } \\
\text { B } \\
\text { C }\end{array}$ & 16.0 & 14.3 & 10.25 & 12.16 & $\begin{array}{c}2.5 \\
- \\
2.5\end{array}$ & 2.5 & $\begin{array}{l}3.3 \\
3.3 \\
3.5\end{array}$ & $\begin{array}{l}5.0 \\
5.0\end{array}$ & 0.375 & 0.11 \\
\hline & (3@7)8-8-T9.5-0-i-2.5-3-9.5 & $\begin{array}{l}\text { A } \\
\text { B } \\
\text { C }\end{array}$ & 19.9 & 14.1 & 10.25 & 12.08 & $\begin{array}{c}2.5 \\
- \\
2.5\end{array}$ & 2.5 & $\begin{array}{l}3.1 \\
2.9 \\
3.2 \\
\end{array}$ & $\begin{array}{l}7.0 \\
6.9\end{array}$ & 0.375 & - \\
\hline & (3@7)8-8-T9.5-2\#3-i-2.5-3-9.5 & $\begin{array}{l}\text { A } \\
\text { B } \\
\text { C }\end{array}$ & 20.1 & 14.3 & 10.25 & 12.05 & $\begin{array}{c}2.5 \\
- \\
2.5\end{array}$ & 2.5 & $\begin{array}{l}3.3 \\
3.0 \\
3.3\end{array}$ & $\begin{array}{l}7.0 \\
7.1\end{array}$ & 0.375 & 0.11 \\
\hline & 8-8-T9.5-0-i-2.5-3-14.5 & $\begin{array}{l}\mathrm{A} \\
\mathrm{B}\end{array}$ & 17.1 & 19.3 & 10.25 & $-\ddagger$ & $\begin{array}{l}2.5 \\
2.5 \\
\end{array}$ & 2.5 & $\begin{array}{l}3.3 \\
3.6 \\
\end{array}$ & 11.1 & 0.375 & - \\
\hline & (3@4)8-8-T9.5-0-i-2.5-3-14.5 & $\begin{array}{l}\text { A } \\
\text { B } \\
\text { C }\end{array}$ & 14.0 & 19.0 & 10.25 & 13.54 & $\begin{array}{c}2.5 \\
- \\
2.5 \\
\end{array}$ & 2.5 & $\begin{array}{l}3.3 \\
2.8 \\
2.8 \\
\end{array}$ & $\begin{array}{l}4.0 \\
4.0 \\
\end{array}$ & 0.375 & - \\
\hline & (3@4)8-8-T9.5-2\#3-i-2.5-3-14.5 & $\begin{array}{l}\text { A } \\
\text { B } \\
\text { C }\end{array}$ & 14.0 & 19.0 & 10.25 & 13.92 & $\begin{array}{c}2.5 \\
- \\
2.5\end{array}$ & 2.5 & $\begin{array}{l}3.0 \\
3.0 \\
3.3\end{array}$ & $\begin{array}{l}4.0 \\
4.0\end{array}$ & 0.375 & 0.11 \\
\hline & (3@5)8-8-T9.5-0-i-2.5-3-14.5 & $\begin{array}{l}\text { A } \\
B \\
C\end{array}$ & 15.8 & 19.1 & 10.25 & 13.43 & $\begin{array}{c}2.5 \\
- \\
2.5\end{array}$ & 2.5 & $\begin{array}{l}2.9 \\
3.1 \\
3.1\end{array}$ & $\begin{array}{l}4.8 \\
5.0\end{array}$ & 0.375 & - \\
\hline & (3@5)8-8-T9.5-2\#3-i-2.5-3-14.5 & $\begin{array}{l}\text { A } \\
\text { B } \\
\text { C }\end{array}$ & 15.5 & 19.3 & 10.25 & 13.84 & $\begin{array}{c}2.3 \\
- \\
2.3\end{array}$ & 2.3 & $\begin{array}{l}3.8 \\
3.5 \\
3.8\end{array}$ & $\begin{array}{l}5.0 \\
5.0\end{array}$ & 0.375 & 0.11 \\
\hline & (3@7)8-8-T9.5-0-i-2.5-3-14.5 & $\begin{array}{l}\text { A } \\
\text { B } \\
\text { C }\end{array}$ & 20.0 & 19.1 & 10.25 & 13.02 & $\begin{array}{c}2.5 \\
- \\
2.5 \\
\end{array}$ & 2.5 & $\begin{array}{l}3.1 \\
3.0 \\
2.9 \\
\end{array}$ & $\begin{array}{l}7.0 \\
7.0 \\
\end{array}$ & 0.375 & - \\
\hline & (3@7)8-8-T9.5-2\#3-i-2.5-3-14.5 & $\begin{array}{l}\text { A } \\
\text { B } \\
\text { C }\end{array}$ & 20.0 & 19.1 & 10.25 & 13.28 & $\begin{array}{c}2.5 \\
- \\
2.5\end{array}$ & 2.5 & $\begin{array}{l}3.1 \\
2.9 \\
3.1\end{array}$ & $\begin{array}{l}7.0 \\
7.0\end{array}$ & 0.375 & 0.11 \\
\hline
\end{tabular}

\footnotetext{
${ }^{\ddagger} d_{\text {eff }}$ was not calculated for specimen with bar yielding
} 
Table A.3 Cont. Comprehensive test results and data for beam-column joint specimens

\begin{tabular}{|c|c|c|c|c|c|c|c|c|c|c|c|}
\hline & Specimen & Head & $N$ & $\begin{array}{l}S_{t r}{ }^{*} \\
\text { in. }\end{array}$ & $\begin{array}{l}\boldsymbol{A}_{\boldsymbol{t t}} \\
\text { in. }^{2}\end{array}$ & $\begin{array}{c}\boldsymbol{d}_{\text {tro }} \\
\text { in. }\end{array}$ & $\begin{array}{c}\text { Stro* } \\
\text { in. }\end{array}$ & $\begin{array}{l}A_{a b} \\
\text { in. }^{2}\end{array}$ & $n$ & $\begin{array}{l}A_{\text {hs }} \\
\text { in. }^{2}\end{array}$ & $\begin{array}{l}\text { Long. } \\
\text { Reinf. } \\
\text { Layout }\end{array}$ \\
\hline \multirow{15}{*}{ Group 9} & 8-8-T9.5-0-i-2.5-3-9.5 & $\begin{array}{l}\text { A } \\
\text { B }\end{array}$ & - & - & - & 0.375 & $\begin{array}{c}3 \\
(1.5) \\
\end{array}$ & 0.66 & 2 & 1.58 & A6 \\
\hline & 8-8-T9.5-2\#3-i-2.5-3-9.5 & $\begin{array}{l}\mathrm{A} \\
\mathrm{B}\end{array}$ & 4 & $\begin{array}{c}6 \\
(4.5) \\
\end{array}$ & 0.22 & 0.375 & $\begin{array}{c}3 \\
(1.5) \\
\end{array}$ & 0.66 & 2 & 1.58 & A6 \\
\hline & (3@4)8-8-T9.5-0-i-2.5-3-9.5 & $\begin{array}{l}\mathrm{A} \\
\mathrm{B} \\
\mathrm{C}\end{array}$ & - & - & - & 0.375 & $\begin{array}{c}3 \\
(1.5)\end{array}$ & 0.66 & 3 & 2.37 & A5 \\
\hline & (3@4)8-8-T9.5-2\#3-i-2.5-3-9.5 & $\begin{array}{l}\text { A } \\
B \\
C\end{array}$ & 4 & $\begin{array}{c}6 \\
(4.5)\end{array}$ & 0.22 & 0.375 & $\begin{array}{c}3 \\
(1.5)\end{array}$ & 0.66 & 3 & 2.37 & A5 \\
\hline & (3@5)8-8-T9.5-0-i-2.5-3-9.5 & $\begin{array}{l}\text { A } \\
\text { B } \\
\text { C }\end{array}$ & - & - & - & 0.375 & $\begin{array}{c}3 \\
(1.5)\end{array}$ & 0.66 & 3 & 2.37 & A5 \\
\hline & (3@5)8-8-T9.5-2\#3-i-2.5-3-9.5 & $\begin{array}{l}\text { A } \\
B \\
C\end{array}$ & 4 & $\begin{array}{c}6 \\
(4.5)\end{array}$ & 0.22 & 0.375 & $\begin{array}{c}3 \\
(1.5)\end{array}$ & 0.66 & 3 & 2.37 & A5 \\
\hline & (3@7)8-8-T9.5-0-i-2.5-3-9.5 & $\begin{array}{l}\text { A } \\
B \\
C\end{array}$ & - & - & - & 0.5 & $\begin{array}{c}4.5 \\
(2.25)\end{array}$ & 0.80 & 3 & 2.37 & A7 \\
\hline & (3@7)8-8-T9.5-2\#3-i-2.5-3-9.5 & $\begin{array}{l}\text { A } \\
B \\
C\end{array}$ & 4 & $\begin{array}{c}6 \\
(4.5)\end{array}$ & 0.22 & 0.5 & $\begin{array}{c}4.5 \\
(2.25)\end{array}$ & 0.80 & 3 & 2.37 & A7 \\
\hline & 8-8-T9.5-0-i-2.5-3-14.5 & $\begin{array}{l}\mathrm{A} \\
\mathrm{B}\end{array}$ & - & - & - & 0.375 & $\begin{array}{c}4 \\
(2) \\
\end{array}$ & 0.28 & 2 & 1.58 & A6 \\
\hline & (3@4)8-8-T9.5-0-i-2.5-3-14.5 & $\begin{array}{l}\mathrm{A} \\
\mathrm{B} \\
\mathrm{C}\end{array}$ & - & - & - & 0.375 & $\begin{array}{c}4 \\
(2)\end{array}$ & 0.44 & 3 & 2.37 & A5 \\
\hline & (3@4)8-8-T9.5-2\#3-i-2.5-3-14.5 & $\begin{array}{l}\mathrm{A} \\
\mathrm{B} \\
\mathrm{C}\end{array}$ & 4 & $\begin{array}{c}6 \\
(4.5)\end{array}$ & 0.22 & 0.375 & $\begin{array}{c}4 \\
(2)\end{array}$ & 0.44 & 3 & 2.37 & A5 \\
\hline & (3@5)8-8-T9.5-0-i-2.5-3-14.5 & $\begin{array}{l}\mathrm{A} \\
\mathrm{B} \\
\mathrm{C}\end{array}$ & - & - & - & 0.375 & $\begin{array}{c}3.5 \\
(1.75)\end{array}$ & 0.44 & 3 & 2.37 & A8 \\
\hline & (3@5)8-8-T9.5-2\#3-i-2.5-3-14.5 & $\begin{array}{l}\text { A } \\
B \\
C\end{array}$ & 4 & $\begin{array}{c}6 \\
(4.5)\end{array}$ & 0.22 & 0.375 & $\begin{array}{c}3.5 \\
(1.75)\end{array}$ & 0.44 & 3 & 2.37 & A8 \\
\hline & (3@7)8-8-T9.5-0-i-2.5-3-14.5 & $\begin{array}{l}\text { A } \\
B \\
C\end{array}$ & - & - & - & 0.5 & $\begin{array}{c}4.5 \\
(2.25)\end{array}$ & 0.80 & 3 & 2.37 & A8 \\
\hline & (3@7)8-8-T9.5-2\#3-i-2.5-3-14.5 & $\begin{array}{l}\text { A } \\
B \\
C\end{array}$ & 4 & $\begin{array}{c}6 \\
(4.5)\end{array}$ & 0.22 & 0.5 & $\begin{array}{c}4.5 \\
(2.25)\end{array}$ & 0.80 & 3 & 2.37 & A8 \\
\hline
\end{tabular}

\footnotetext{
${ }^{*}$ Value in parenthesis is the spacing between the first hoop and the center of the headed bar
} 
Table A.3 Cont. Comprehensive test results and data for beam-column joint specimens

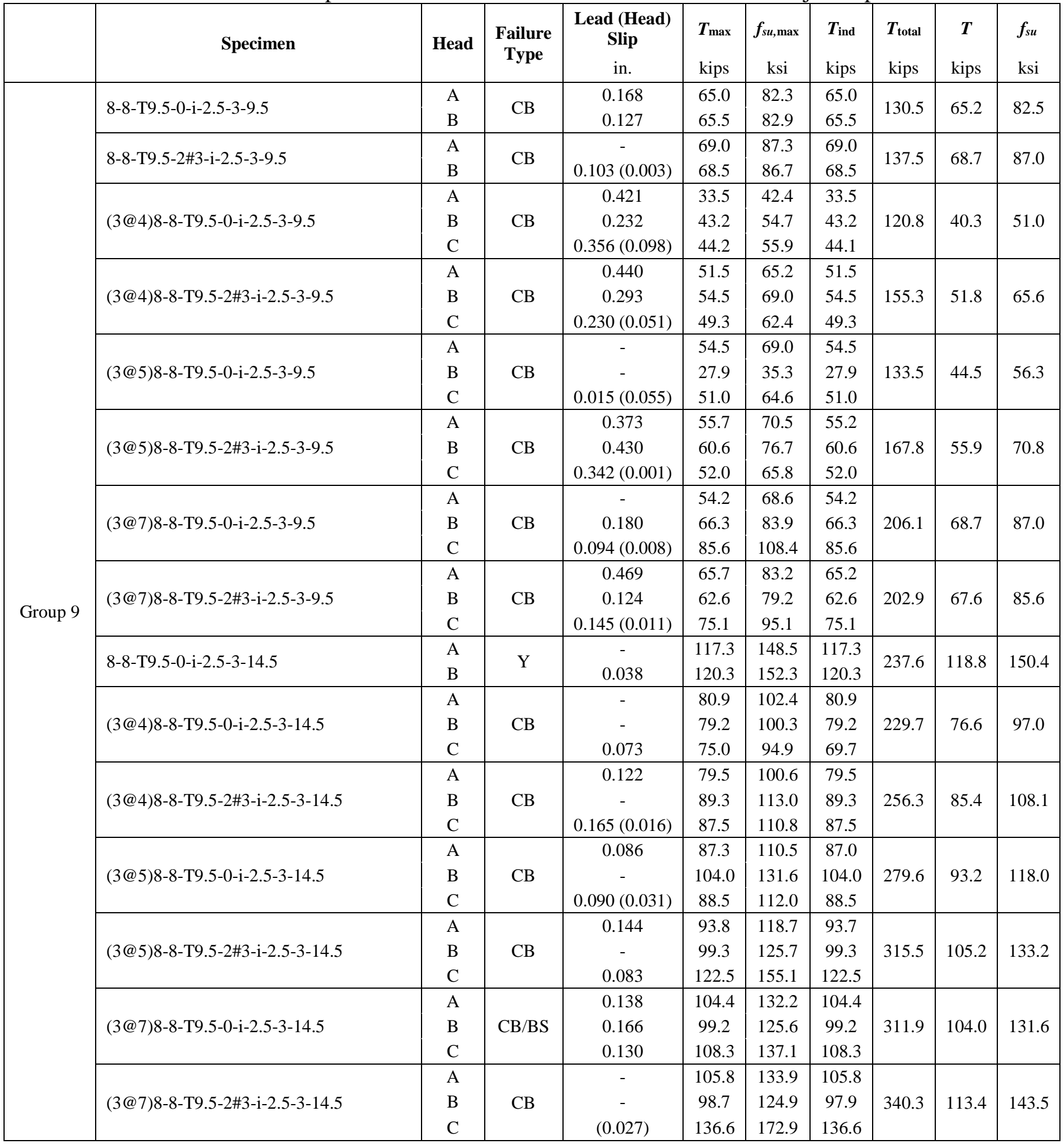


Table A.3 Cont. Comprehensive test results and data for beam-column joint specimens

\begin{tabular}{|c|c|c|c|c|c|c|c|c|c|c|}
\hline & Specimen & Head & $\begin{array}{l}\boldsymbol{c}_{\boldsymbol{o}} \\
\text { in. }\end{array}$ & $A_{b r g}$ & $\begin{array}{l}\ell e h \\
\text { in. }\end{array}$ & $\begin{array}{c}\ell \text { eh,avg } \\
\text { in. }\end{array}$ & $\begin{array}{l}f_{c m} \\
\text { psi }\end{array}$ & $\begin{array}{l}\text { Age } \\
\text { days }\end{array}$ & $\begin{array}{l}\boldsymbol{d}_{b} \\
\text { in. }\end{array}$ & $\begin{array}{l}\boldsymbol{A}_{\boldsymbol{b}} \\
\text { in. }^{2}\end{array}$ \\
\hline \multirow{12}{*}{ Group 10} & (2@9)8-12-F4.1-0-i-2.5-3-12 & $\begin{array}{l}\text { A } \\
B\end{array}$ & 2.0 & $4.1 A_{b}$ & $\begin{array}{l}12.13 \\
12.00\end{array}$ & 12.06 & 12080 & 57 & 1 & 0.79 \\
\hline & (2@9)8-12-F9.1-0-i-2.5-3-12 & $\begin{array}{l}\text { A } \\
B\end{array}$ & 2.0 & $9.1 A_{b}$ & $\begin{array}{l}11.75 \\
12.00\end{array}$ & 11.88 & 12080 & 57 & 1 & 0.79 \\
\hline & (2@9)8-12-F4.1-5\#3-i-2.5-3-12 & $\begin{array}{l}\mathrm{A} \\
\mathrm{B}\end{array}$ & 2.0 & $4.1 A_{b}$ & $\begin{array}{l}11.94 \\
12.00\end{array}$ & 11.97 & 12080 & 57 & 1 & 0.79 \\
\hline & (2@9)8-12-F9.1-5\#3-i-2.5-3-12 & $\begin{array}{l}\text { A } \\
\text { B }\end{array}$ & 2.0 & $9.1 A_{b}$ & $\begin{array}{l}12.13 \\
12.13\end{array}$ & 12.13 & 12080 & 57 & 1 & 0.79 \\
\hline & (3@4.5)8-12-F4.1-0-i-2.5-3-12 & $\begin{array}{l}\text { A } \\
B \\
C\end{array}$ & 2.0 & $4.1 A_{b}$ & $\begin{array}{l}12.13 \\
12.25 \\
12.25\end{array}$ & 12.21 & 12040 & 58 & 1 & 0.79 \\
\hline & (3@4.5)8-12-F9.1-0-i-2.5-3-12 & $\begin{array}{l}\text { A } \\
B \\
C\end{array}$ & 2.0 & $9.1 A_{b}$ & $\begin{array}{l}12.00 \\
12.13 \\
12.00 \\
\end{array}$ & 12.04 & 12040 & 58 & 1 & 0.79 \\
\hline & (3@4.5)8-12-F4.1-5\#3-i-2.5-3-12 & $\begin{array}{l}\text { A } \\
\text { B } \\
\text { C }\end{array}$ & 2.0 & $4.1 A_{b}$ & $\begin{array}{l}12.13 \\
12.19 \\
12.19 \\
\end{array}$ & 12.17 & 12040 & 58 & 1 & 0.79 \\
\hline & (3@4.5)8-12-F9.1-5\#3-i-2.5-3-12 & $\begin{array}{l}\text { A } \\
B \\
C\end{array}$ & 2.0 & $9.1 A_{b}$ & $\begin{array}{l}11.94 \\
11.88 \\
11.88\end{array}$ & 11.90 & 12040 & 58 & 1 & 0.79 \\
\hline & (4@3)8-12-F4.1-0-i-2.5-3-12 & $\begin{array}{l}A \\
B \\
C \\
D \\
\end{array}$ & 2.0 & $4.1 A_{b}$ & $\begin{array}{l}12.00 \\
12.00 \\
12.00 \\
12.00 \\
\end{array}$ & 12.00 & 12040 & 58 & 1 & 0.79 \\
\hline & (4@3)8-12-F9.1-0-i-2.5-3-12 & $\begin{array}{l}\mathrm{A} \\
\mathrm{B} \\
\mathrm{C} \\
\mathrm{D}\end{array}$ & 2.0 & $9.1 A_{b}$ & $\begin{array}{l}12.06 \\
12.13 \\
12.25 \\
12.25 \\
\end{array}$ & 12.17 & 12360 & 61 & 1 & 0.79 \\
\hline & (4@3)8-12-F4.1-5\#3-i-2.5-3-12 & $\begin{array}{l}\mathrm{A} \\
\mathrm{B} \\
\mathrm{C} \\
\mathrm{D} \\
\end{array}$ & 2.0 & $4.1 A_{b}$ & $\begin{array}{l}12.00 \\
12.00 \\
12.13 \\
12.00 \\
\end{array}$ & 12.03 & 12360 & 61 & 1 & 0.79 \\
\hline & (4@3)8-12-F9.1-5\#3-i-2.5-3-12 & $\begin{array}{l}\text { A } \\
B \\
C \\
D\end{array}$ & 2.0 & $9.1 A_{b}$ & $\begin{array}{l}12.00 \\
12.00 \\
12.00 \\
11.81\end{array}$ & 11.95 & 12360 & 61 & 1 & 0.79 \\
\hline \multirow{4}{*}{ Group 11} & 8-8-O4.5-0-i-2.5-3-9.5 & $\begin{array}{l}\text { A } \\
\text { B }\end{array}$ & 1.6 & $4.5 A_{b}$ & $\begin{array}{l}9.13 \\
9.25 \\
\end{array}$ & 9.19 & 6710 & 16 & 1 & 0.79 \\
\hline & (2@9)8-8-O4.5-0-i-2.5-3-9.5 & $\begin{array}{l}\mathrm{A} \\
\mathrm{B}\end{array}$ & 1.6 & $4.5 A_{b}$ & $\begin{array}{l}9.13 \\
8.88 \\
\end{array}$ & 9.00 & 6710 & 16 & 1 & 0.79 \\
\hline & (2@7)8-8-O4.5-0-i-2.5-3-9.5 & $\begin{array}{l}\text { A } \\
B\end{array}$ & 1.6 & $4.5 A_{b}$ & $\begin{array}{l}9.38 \\
9.13\end{array}$ & 9.25 & 6710 & 16 & 1 & 0.79 \\
\hline & (2@5)8-8-O4.5-0-i-2.5-3-9.5 & $\begin{array}{l}\mathrm{A} \\
\mathrm{B}\end{array}$ & 1.6 & $4.5 A_{b}$ & $\begin{array}{l}9.13 \\
8.88 \\
\end{array}$ & 9.00 & 6710 & 16 & 1 & 0.79 \\
\hline
\end{tabular}


Table A.3 Cont. Comprehensive test results and data for beam-column joint specimens

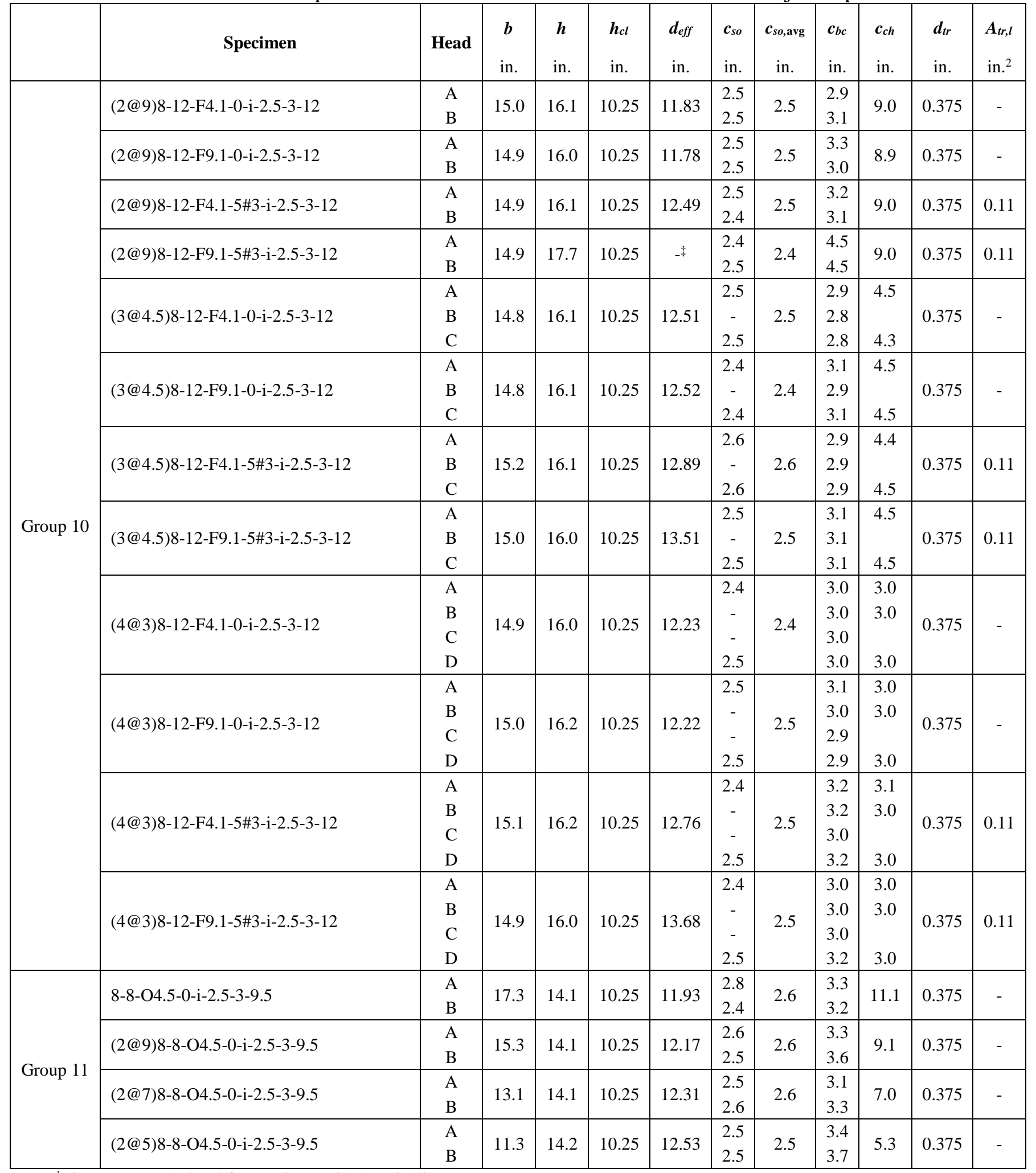

\footnotetext{
${ }^{\ddagger} d_{\text {eff }}$ was not calculated for specimen with bar yielding
} 
Table A.3 Cont. Comprehensive test results and data for beam-column joint specimens

\begin{tabular}{|c|c|c|c|c|c|c|c|c|c|c|c|}
\hline & Specimen & Head & $N$ & $\begin{array}{l}\boldsymbol{s}_{t r}^{*} \\
\text { in. }\end{array}$ & $\begin{array}{l}\boldsymbol{A}_{t t} \\
\text { in. }^{2}\end{array}$ & $\begin{array}{l}\boldsymbol{d}_{\text {tro }} \\
\text { in. }\end{array}$ & $\begin{array}{l}\text { Stro }^{*} \\
\text { in. }\end{array}$ & $\begin{array}{l}A_{a b} \\
\text { in. }^{2}\end{array}$ & $n$ & $\begin{array}{l}A_{h s} \\
\text { in. }^{2}\end{array}$ & $\begin{array}{l}\text { Long. } \\
\text { Reinf. } \\
\text { Layout }\end{array}$ \\
\hline \multirow{12}{*}{ Group 10} & (2@9)8-12-F4.1-0-i-2.5-3-12 & $\begin{array}{l}\mathrm{A} \\
\mathrm{B}\end{array}$ & - & - & - & 0.5 & $\begin{array}{c}3 \\
(1.5) \\
\end{array}$ & 1.20 & 2 & 1.58 & A5 \\
\hline & (2@9)8-12-F9.1-0-i-2.5-3-12 & $\begin{array}{l}\text { A } \\
B\end{array}$ & - & - & - & 0.5 & $\begin{array}{c}3 \\
(1.5)\end{array}$ & 1.20 & 2 & 1.58 & A5 \\
\hline & (2@9)8-12-F4.1-5\#3-i-2.5-3-12 & $\begin{array}{l}\text { A } \\
B\end{array}$ & 10 & $\begin{array}{c}3 \\
(1.5) \\
\end{array}$ & 0.66 & 0.5 & $\begin{array}{c}3 \\
(1.5)\end{array}$ & 1.20 & 2 & 1.58 & A5 \\
\hline & (2@9)8-12-F9.1-5\#3-i-2.5-3-12 & $\begin{array}{l}\mathrm{A} \\
\mathrm{B}\end{array}$ & 10 & $\begin{array}{c}3 \\
(1.5) \\
\end{array}$ & 0.66 & 0.5 & $\begin{array}{c}3 \\
(1.5) \\
\end{array}$ & 0.76 & 2 & 1.58 & A5 \\
\hline & (3@4.5)8-12-F4.1-0-i-2.5-3-12 & $\begin{array}{l}\text { A } \\
\text { B } \\
\text { C }\end{array}$ & - & - & - & 0.5 & $\begin{array}{c}3 \\
(1.5)\end{array}$ & 1.20 & 3 & 2.37 & A5 \\
\hline & (3@4.5)8-12-F9.1-0-i-2.5-3-12 & $\begin{array}{l}\text { A } \\
B \\
C\end{array}$ & - & - & - & 0.5 & $\begin{array}{c}3 \\
(1.5)\end{array}$ & 1.20 & 3 & 2.37 & A5 \\
\hline & (3@4.5)8-12-F4.1-5\#3-i-2.5-3-12 & $\begin{array}{l}\text { A } \\
B \\
C\end{array}$ & 10 & $\begin{array}{c}3 \\
(1.5)\end{array}$ & 0.66 & 0.5 & $\begin{array}{c}3 \\
(1.5)\end{array}$ & 1.20 & 3 & 2.37 & A5 \\
\hline & (3@4.5)8-12-F9.1-5\#3-i-2.5-3-12 & $\begin{array}{l}\text { A } \\
\text { B } \\
\text { C }\end{array}$ & 10 & $\begin{array}{c}3 \\
(1.5)\end{array}$ & 0.66 & 0.5 & $\begin{array}{c}3 \\
(1.5)\end{array}$ & 1.20 & 3 & 2.37 & A5 \\
\hline & (4@3)8-12-F4.1-0-i-2.5-3-12 & $\begin{array}{l}\mathrm{A} \\
\mathrm{B} \\
\mathrm{C} \\
\mathrm{D}\end{array}$ & - & - & - & 0.5 & $\begin{array}{c}3 \\
(1.5)\end{array}$ & 1.20 & 4 & 3.16 & A5 \\
\hline & (4@3)8-12-F9.1-0-i-2.5-3-12 & $\begin{array}{l}\mathrm{A} \\
\mathrm{B} \\
\mathrm{C} \\
\mathrm{D}\end{array}$ & - & - & - & 0.5 & $\begin{array}{c}3 \\
(1.5)\end{array}$ & 1.20 & 4 & 3.16 & A5 \\
\hline & (4@3)8-12-F4.1-5\#3-i-2.5-3-12 & $\begin{array}{l}\text { A } \\
\text { B } \\
\text { C } \\
\text { D }\end{array}$ & 10 & $\begin{array}{c}3 \\
(1.5)\end{array}$ & 0.66 & 0.5 & $\begin{array}{c}3 \\
(1.5)\end{array}$ & 1.20 & 4 & 3.16 & A5 \\
\hline & (4@3)8-12-F9.1-5\#3-i-2.5-3-12 & $\begin{array}{l}\mathrm{A} \\
\mathrm{B} \\
\mathrm{C} \\
\mathrm{D}\end{array}$ & 10 & $\begin{array}{c}3 \\
(1.5)\end{array}$ & 0.66 & 0.5 & $\begin{array}{c}3 \\
(1.5)\end{array}$ & 1.20 & 4 & 3.16 & A5 \\
\hline \multirow{4}{*}{ Group 11} & 8-8-O4.5-0-i-2.5-3-9.5 & $\begin{array}{l}\mathrm{A} \\
\mathrm{B}\end{array}$ & - & - & - & 0.375 & $\begin{array}{c}4 \\
(2) \\
\end{array}$ & 0.44 & 2 & 1.58 & A4 \\
\hline & (2@9)8-8-04.5-0-i-2.5-3-9.5 & $\begin{array}{l}\mathrm{A} \\
\mathrm{B}\end{array}$ & - & - & - & 0.375 & $\begin{array}{c}4 \\
(2) \\
\end{array}$ & 0.44 & 2 & 1.58 & A4 \\
\hline & (2@7)8-8-O4.5-0-i-2.5-3-9.5 & $\begin{array}{l}\mathrm{A} \\
\mathrm{B}\end{array}$ & - & - & - & 0.375 & $\begin{array}{c}4 \\
(2) \\
\end{array}$ & 0.44 & 2 & 1.58 & A4 \\
\hline & (2@5)8-8-O4.5-0-i-2.5-3-9.5 & $\begin{array}{l}A \\
B\end{array}$ & - & - & - & 0.375 & $\begin{array}{c}4 \\
(2) \\
\end{array}$ & 0.44 & 2 & 1.58 & A4 \\
\hline
\end{tabular}

${ }^{*}$ Value in parenthesis is the spacing between the first hoop and the center of the headed bar 
Table A.3 Cont. Comprehensive test results and data for beam-column joint specimens

\begin{tabular}{|c|c|c|c|c|c|c|c|c|c|c|}
\hline & Specimen & Head & $\begin{array}{c}\text { Failure } \\
\text { Type }\end{array}$ & $\begin{array}{c}\text { Lead (Head) } \\
\text { Slip } \\
\text { in. }\end{array}$ & $\begin{array}{l}\boldsymbol{T}_{\text {max }} \\
\text { kips }\end{array}$ & $\begin{array}{c}\boldsymbol{f}_{\text {su,max }} \\
\text { ksi }\end{array}$ & $\begin{array}{l}\boldsymbol{T}_{\text {ind }} \\
\text { kips }\end{array}$ & $\begin{array}{l}\boldsymbol{T}_{\text {total }} \\
\text { kips }\end{array}$ & $\begin{array}{c}\boldsymbol{T} \\
\text { kips }\end{array}$ & $\begin{array}{l}\boldsymbol{f}_{\text {su }} \\
\mathrm{ksi}\end{array}$ \\
\hline \multirow{12}{*}{ Group 10} & (2@9)8-12-F4.1-0-i-2.5-3-12 & $\begin{array}{l}\text { A } \\
B\end{array}$ & $\mathrm{CB} / \mathrm{FP}$ & $\begin{array}{l}- \\
-\end{array}$ & $\begin{array}{l}79.8 \\
78.3\end{array}$ & $\begin{array}{c}101.0 \\
99.1\end{array}$ & $\begin{array}{l}79.8 \\
78.3\end{array}$ & 158.1 & 79.1 & 100.1 \\
\hline & (2@9)8-12-F9.1-0-i-2.5-3-12 & $\begin{array}{l}\text { A } \\
\text { B }\end{array}$ & $\mathrm{CB} / \mathrm{BS}$ & $\begin{array}{c}0.048 \\
-\end{array}$ & $\begin{array}{l}76.1 \\
76.9\end{array}$ & $\begin{array}{l}96.3 \\
97.3\end{array}$ & $\begin{array}{l}76.1 \\
76.9\end{array}$ & 153.0 & 76.5 & 96.8 \\
\hline & (2@9)8-12-F4.1-5\#3-i-2.5-3-12 & $\begin{array}{l}\text { A } \\
\text { B }\end{array}$ & $\mathrm{SB} / \mathrm{FP}$ & $\begin{array}{l}0.126 \\
0.126\end{array}$ & $\begin{array}{l}112.5^{\ddagger} \\
111.3\end{array}$ & $\begin{array}{l}142.4^{\ddagger} \\
140.9\end{array}$ & $\begin{array}{l}112.5 \\
111.3\end{array}$ & 223.8 & 111.9 & 141.6 \\
\hline & (2@9)8-12-F9.1-5\#3-i-2.5-3-12 & $\begin{array}{l}\text { A } \\
\text { B }\end{array}$ & $\mathrm{Y}$ & $\begin{array}{l}0.200 \\
0.025\end{array}$ & $\begin{array}{l}125.2 \\
117.1\end{array}$ & $\begin{array}{l}158.5 \\
148.2\end{array}$ & $\begin{array}{l}125.2 \\
117.1\end{array}$ & 242.3 & 121.2 & 153.4 \\
\hline & (3@4.5)8-12-F4.1-0-i-2.5-3-12 & $\begin{array}{l}\text { A } \\
B \\
C\end{array}$ & CB & $\begin{array}{l}0.133 \\
0.037 \\
0.089 \\
\end{array}$ & $\begin{array}{l}79.1 \\
75.8 \\
70.7 \\
\end{array}$ & $\begin{array}{c}100.1 \\
95.9 \\
89.5 \\
\end{array}$ & $\begin{array}{l}79.1 \\
75.8 \\
70.7 \\
\end{array}$ & 225.7 & 75.2 & 95.2 \\
\hline & (3@4.5)8-12-F9.1-0-i-2.5-3-12 & $\begin{array}{l}\text { A } \\
\text { B } \\
\text { C }\end{array}$ & CB & $\begin{array}{c}(0.046) \\
- \\
0.117\end{array}$ & $\begin{array}{l}77.8 \\
63.3 \\
85.1 \\
\end{array}$ & $\begin{array}{c}98.5 \\
80.1 \\
107.7 \\
\end{array}$ & $\begin{array}{l}77.8 \\
63.3 \\
85.1 \\
\end{array}$ & 226.2 & 75.4 & 95.4 \\
\hline & (3@4.5)8-12-F4.1-5\#3-i-2.5-3-12 & $\begin{array}{l}\text { A } \\
\text { B } \\
\text { C }\end{array}$ & CB & $\begin{array}{l}0.170 \\
0.094 \\
0.169 \\
\end{array}$ & $\begin{array}{l}83.8 \\
86.0 \\
93.2\end{array}$ & $\begin{array}{l}106.1 \\
108.9 \\
118.0 \\
\end{array}$ & $\begin{array}{l}83.8 \\
86.0 \\
93.2 \\
\end{array}$ & 263.1 & 87.7 & 111.0 \\
\hline & (3@4.5)8-12-F9.1-5\#3-i-2.5-3-12 & $\begin{array}{l}\text { A } \\
\text { B } \\
\text { C }\end{array}$ & CB & $\begin{array}{l}0.250 \\
0.096 \\
0.234\end{array}$ & $\begin{array}{l}108.1 \\
110.7 \\
106.9\end{array}$ & $\begin{array}{l}136.8 \\
140.1 \\
135.3\end{array}$ & $\begin{array}{l}108.1 \\
110.7 \\
106.9\end{array}$ & 325.7 & 108.6 & 137.4 \\
\hline & (4@3)8-12-F4.1-0-i-2.5-3-12 & $\begin{array}{l}A \\
B \\
C \\
D\end{array}$ & CB & $\begin{array}{c}- \\
- \\
0.135 \\
0.032\end{array}$ & $\begin{array}{l}41.7 \\
49.5 \\
66.8 \\
39.4\end{array}$ & $\begin{array}{l}52.8 \\
62.7 \\
84.6 \\
49.9\end{array}$ & $\begin{array}{l}41.7 \\
49.5 \\
66.8 \\
39.4\end{array}$ & 197.2 & 49.3 & 62.4 \\
\hline & (4@3)8-12-F9.1-0-i-2.5-3-12 & $\begin{array}{l}\text { A } \\
\text { B } \\
\text { C } \\
\text { D }\end{array}$ & CB & $\begin{array}{l}- \\
- \\
- \\
-\end{array}$ & $\begin{array}{l}49.2 \\
45.7 \\
53.2 \\
53.1 \\
\end{array}$ & $\begin{array}{l}62.3 \\
57.8 \\
67.3 \\
67.2 \\
\end{array}$ & $\begin{array}{l}49.2 \\
45.7 \\
53.2 \\
53.1 \\
\end{array}$ & 201.3 & 50.3 & 63.7 \\
\hline & (4@3)8-12-F4.1-5\#3-i-2.5-3-12 & $\begin{array}{l}\text { A } \\
\text { B } \\
\text { C } \\
\text { D }\end{array}$ & CB & $\begin{array}{c}0.030 \\
- \\
0.101 \\
0.093\end{array}$ & $\begin{array}{l}73.8 \\
63.3 \\
48.2 \\
71.5 \\
\end{array}$ & $\begin{array}{l}93.4 \\
80.1 \\
61.0 \\
90.5 \\
\end{array}$ & $\begin{array}{l}73.8 \\
63.3 \\
48.2 \\
71.5 \\
\end{array}$ & 256.7 & 64.2 & 81.2 \\
\hline & (4@3)8-12-F9.1-5\#3-i-2.5-3-12 & $\begin{array}{l}\text { A } \\
\text { B } \\
\text { C } \\
\text { D }\end{array}$ & CB & $\begin{array}{l}- \\
- \\
- \\
-\end{array}$ & $\begin{array}{c}85.2 \\
72.8 \\
111.1 \\
82.1\end{array}$ & $\begin{array}{c}107.8 \\
92.2 \\
140.6 \\
103.9\end{array}$ & $\begin{array}{c}85.2 \\
72.8 \\
111.1 \\
82.1 \\
\end{array}$ & 351.3 & 87.8 & 111.1 \\
\hline \multirow{4}{*}{ Group 11} & 8-8-O4.5-0-i-2.5-3-9.5 & $\begin{array}{l}\mathrm{A} \\
\mathrm{B}\end{array}$ & $\mathrm{CB} / \mathrm{FP}$ & $\begin{array}{l}0.002 \\
0.002\end{array}$ & $\begin{array}{l}61.9 \\
54.9 \\
\end{array}$ & $\begin{array}{l}78.4 \\
69.5 \\
\end{array}$ & $\begin{array}{l}61.8 \\
54.9 \\
\end{array}$ & 116.7 & 58.4 & 73.9 \\
\hline & (2@9)8-8-O4.5-0-i-2.5-3-9.5 & $\begin{array}{l}\mathrm{A} \\
\mathrm{B}\end{array}$ & CB & $\begin{array}{l}0.014 \\
0.019 \\
\end{array}$ & $\begin{array}{l}57.5 \\
60.1 \\
\end{array}$ & $\begin{array}{l}72.8 \\
76.1 \\
\end{array}$ & $\begin{array}{l}57.5 \\
60.1 \\
\end{array}$ & 117.6 & 58.8 & 74.4 \\
\hline & (2@7)8-8-04.5-0-i-2.5-3-9.5 & $\begin{array}{l}\mathrm{A} \\
\mathrm{B}\end{array}$ & CB & $\begin{array}{l}0.010 \\
0.030 \\
\end{array}$ & $\begin{array}{l}57.2 \\
51.8 \\
\end{array}$ & $\begin{array}{l}72.4 \\
65.6 \\
\end{array}$ & $\begin{array}{l}57.2 \\
51.8 \\
\end{array}$ & 109.0 & 54.5 & 69.0 \\
\hline & (2@5)8-8-O4.5-0-i-2.5-3-9.5 & $\begin{array}{l}\text { A } \\
\text { B }\end{array}$ & CB & $\begin{array}{l}0.035 \\
0.041\end{array}$ & $\begin{array}{l}45.7 \\
56.7\end{array}$ & $\begin{array}{l}57.8 \\
71.8\end{array}$ & $\begin{array}{l}45.7 \\
56.7\end{array}$ & 102.4 & 51.2 & 64.8 \\
\hline
\end{tabular}

\footnotetext{
${ }^{\ddagger}$ No anchorage failure on the bar
} 
Table A.3 Cont. Comprehensive test results and data for beam-column joint specimens

\begin{tabular}{|c|c|c|c|c|c|c|c|c|c|c|}
\hline & Specimen & Head & $\begin{array}{l}c_{o} \\
\text { in. }\end{array}$ & $A_{b r g}$ & $\begin{array}{l}\ell_{\text {eh }} \\
\text { in. }\end{array}$ & $\begin{array}{c}\ell_{\text {eh, avg }} \\
\text { in. }\end{array}$ & $\begin{array}{l}\boldsymbol{f}_{\boldsymbol{c m}} \\
\mathrm{psi}\end{array}$ & $\begin{array}{l}\text { Age } \\
\text { days }\end{array}$ & $\begin{array}{l}d_{b} \\
\text { in. }\end{array}$ & $\begin{array}{l}\boldsymbol{A}_{\boldsymbol{b}} \\
\text { in. }^{2}\end{array}$ \\
\hline \multirow{9}{*}{ Group 11} & (2@3)8-8-O4.5-0-i-2.5-3-9.5 & $\begin{array}{l}\text { A } \\
B\end{array}$ & 1.6 & $4.5 A_{b}$ & $\begin{array}{l}9.13 \\
8.88\end{array}$ & 9.00 & 6710 & 16 & 1 & 0.79 \\
\hline & (2@9)8-8-T4.0-0-i-2.5-3-9.5 & $\begin{array}{l}\text { A } \\
B\end{array}$ & 1.9 & $4.0 A_{b}$ & $\begin{array}{l}9.25 \\
9.50\end{array}$ & 9.38 & 6790 & 17 & 1 & 0.79 \\
\hline & (2@9)8-8-T4.0-5\#3-i-2.5-3-9.5 & $\begin{array}{l}\mathrm{A} \\
\mathrm{B}\end{array}$ & 1.9 & $4.0 A_{b}$ & $\begin{array}{l}9.50 \\
9.50\end{array}$ & 9.50 & 6790 & 17 & 1 & 0.79 \\
\hline & (3@4.5)8-8-T4.0-0-i-2.5-3-9.5 & $\begin{array}{l}A \\
B \\
C\end{array}$ & 1.9 & $4.0 A_{b}$ & $\begin{array}{l}9.25 \\
9.50 \\
9.25\end{array}$ & 9.33 & 6790 & 17 & 1 & 0.79 \\
\hline & (3@4.5)8-8-T4.0-5\#3-i-2.5-3-9.5 & $\begin{array}{l}\text { A } \\
B \\
C\end{array}$ & 1.9 & $4.0 A_{b}$ & $\begin{array}{l}9.13 \\
9.25 \\
9.13\end{array}$ & 9.17 & 6650 & 20 & 1 & 0.79 \\
\hline & (4@3)8-8-T4.0-0-i-2.5-3-9.5 & $\begin{array}{l}A \\
B \\
C \\
D\end{array}$ & 1.9 & $4.0 A_{b}$ & $\begin{array}{l}9.63 \\
9.63 \\
9.25 \\
9.38\end{array}$ & 9.47 & 6650 & 20 & 1 & 0.79 \\
\hline & (4@3)8-8-T4.0-5\#3-i-2.5-3-9.5 & $\begin{array}{l}A \\
B \\
C \\
D\end{array}$ & 1.9 & $4.0 A_{b}$ & $\begin{array}{l}9.75 \\
9.63 \\
9.88 \\
9.38\end{array}$ & 9.66 & 6650 & 20 & 1 & 0.79 \\
\hline & (3@3)8-8-T4.0-0-i-2.5-3-9.5 & $\begin{array}{l}\text { A } \\
B \\
C\end{array}$ & 1.9 & $4.0 A_{b}$ & $\begin{array}{l}9.25 \\
9.63 \\
9.50\end{array}$ & 9.46 & 6790 & 17 & 1 & 0.79 \\
\hline & (3@3)8-8-T4.0-5\#3-i-2.5-3-9.5 & $\begin{array}{l}\text { A } \\
B \\
C \\
\end{array}$ & 1.9 & $4.0 A_{b}$ & $\begin{array}{l}9.25 \\
9.38 \\
9.38 \\
\end{array}$ & 9.33 & 6650 & 20 & 1 & 0.79 \\
\hline \multirow{7}{*}{ Group 12} & 8-5-F4.1-0-i-2.5-7-6 & $\begin{array}{l}\text { A } \\
B\end{array}$ & 2.0 & $4.1 A_{b}$ & $\begin{array}{l}6.06 \\
6.13\end{array}$ & 6.09 & 4930 & 14 & 1 & 0.79 \\
\hline & 8-5-F4.1-5\#3-i-2.5-7-6 & $\begin{array}{l}A \\
B\end{array}$ & 2.0 & $4.1 A_{b}$ & $\begin{array}{l}6.25 \\
6.25\end{array}$ & 6.25 & 4930 & 14 & 1 & 0.79 \\
\hline & (3@3)8-5-F4.1-0-i-2.5-7-6 & $\begin{array}{l}\text { A } \\
\text { B } \\
\text { C }\end{array}$ & 2.0 & $4.1 A_{b}$ & $\begin{array}{l}6.06 \\
6.25 \\
6.25 \\
\end{array}$ & 6.19 & 4930 & 14 & 1 & 0.79 \\
\hline & (3@3)8-5-F4.1-5\#3-i-2.5-7-6 & $\begin{array}{l}\text { A } \\
\text { B } \\
\text { C } \\
\end{array}$ & 2.0 & $4.1 A_{b}$ & $\begin{array}{l}6.00 \\
6.00 \\
6.00 \\
\end{array}$ & 6.00 & 4930 & 14 & 1 & 0.79 \\
\hline & (3@5)8-5-F4.1-0-i-2.5-7-6 & $\begin{array}{l}\text { A } \\
B \\
C\end{array}$ & 2.0 & $4.1 A_{b}$ & $\begin{array}{l}6.50 \\
6.25 \\
6.25\end{array}$ & 6.33 & 4930 & 14 & 1 & 0.79 \\
\hline & (3@5)8-5-F4.1-5\#3-i-2.5-7-6 & $\begin{array}{l}\text { A } \\
B \\
C\end{array}$ & 2.0 & $4.1 A_{b}$ & $\begin{array}{l}6.25 \\
6.13 \\
6.50\end{array}$ & 6.29 & 4930 & 14 & 1 & 0.79 \\
\hline & (3@7)8-5-F4.1-0-i-2.5-7-6 & $\begin{array}{l}\text { A } \\
B \\
C\end{array}$ & 2.0 & $4.1 A_{b}$ & $\begin{array}{l}6.25 \\
6.25 \\
6.25 \\
\end{array}$ & 6.25 & 4940 & 15 & 1 & 0.79 \\
\hline
\end{tabular}


Table A.3 Cont. Comprehensive test results and data for beam-column joint specimens

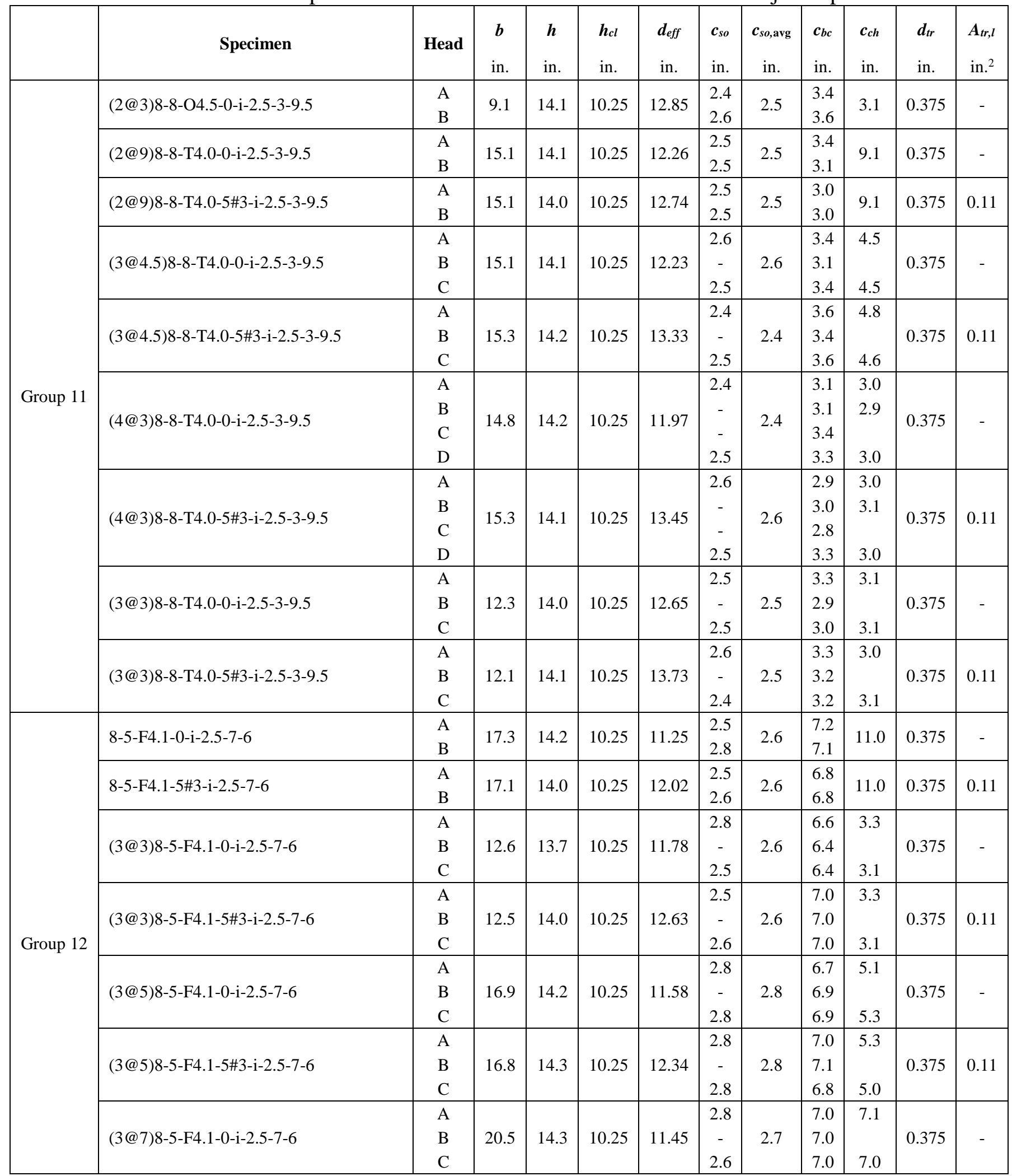


Table A.3 Cont. Comprehensive test results and data for beam-column joint specimens

\begin{tabular}{|c|c|c|c|c|c|c|c|c|c|c|c|}
\hline & Specimen & Head & $N$ & $\begin{array}{l}\boldsymbol{s}_{t r}^{*} \\
\text { in. }\end{array}$ & $\begin{array}{l}\boldsymbol{A}_{\boldsymbol{t t}} \\
\text { in. }^{2}\end{array}$ & $\begin{array}{l}\boldsymbol{d}_{\text {tro }} \\
\text { in. }\end{array}$ & $\begin{array}{l}\text { stro }^{*} \\
\text { in. }\end{array}$ & $\begin{array}{l}A_{a b} \\
\text { in. }^{2}\end{array}$ & $n$ & $\begin{array}{l}\text { A }_{\text {hs }} \\
\text { in. }^{2}\end{array}$ & $\begin{array}{l}\text { Long. } \\
\text { Reinf. } \\
\text { Layout }\end{array}$ \\
\hline \multirow{9}{*}{ Group 11} & (2@3)8-8-O4.5-0-i-2.5-3-9.5 & $\begin{array}{l}\text { A } \\
B\end{array}$ & - & - & - & 0.375 & $\begin{array}{c}4 \\
(2)\end{array}$ & 0.44 & 2 & 1.58 & A4 \\
\hline & (2@9)8-8-T4.0-0-i-2.5-3-9.5 & $\begin{array}{l}\mathrm{A} \\
\mathrm{B}\end{array}$ & - & - & - & 0.5 & $\begin{array}{c}4 \\
(2) \\
\end{array}$ & 0.80 & 2 & 1.58 & A9 \\
\hline & (2@9)8-8-T4.0-5\#3-i-2.5-3-9.5 & $\begin{array}{l}\mathrm{A} \\
\mathrm{B}\end{array}$ & 10 & $\begin{array}{c}3 \\
(1.5) \\
\end{array}$ & 0.66 & 0.5 & $\begin{array}{c}4 \\
(2)\end{array}$ & 0.80 & 2 & 1.58 & A5 \\
\hline & (3@4.5)8-8-T4.0-0-i-2.5-3-9.5 & $\begin{array}{l}\mathrm{A} \\
\mathrm{B} \\
\mathrm{C}\end{array}$ & - & - & - & 0.5 & $\begin{array}{c}4 \\
(2)\end{array}$ & 0.80 & 3 & 2.37 & A9 \\
\hline & (3@4.5)8-8-T4.0-5\#3-i-2.5-3-9.5 & $\begin{array}{l}\mathrm{A} \\
\mathrm{B} \\
\mathrm{C}\end{array}$ & 10 & $\begin{array}{c}3 \\
(1.5)\end{array}$ & 0.66 & 0.5 & $\begin{array}{c}4 \\
(2)\end{array}$ & 0.80 & 3 & 2.37 & A5 \\
\hline & (4@3)8-8-T4.0-0-i-2.5-3-9.5 & $\begin{array}{l}\mathrm{A} \\
\mathrm{B} \\
\mathrm{C} \\
\mathrm{D}\end{array}$ & - & - & - & 0.5 & $\begin{array}{c}4 \\
(2)\end{array}$ & 0.80 & 4 & 3.16 & A9 \\
\hline & (4@3)8-8-T4.0-5\#3-i-2.5-3-9.5 & $\begin{array}{l}\text { A } \\
\text { B } \\
\text { C } \\
\text { D }\end{array}$ & 10 & $\begin{array}{c}3 \\
(1.5)\end{array}$ & 0.66 & 0.5 & $\begin{array}{c}4 \\
(2)\end{array}$ & 0.80 & 4 & 3.16 & A5 \\
\hline & (3@3)8-8-T4.0-0-i-2.5-3-9.5 & $\begin{array}{l}\text { A } \\
\text { B } \\
\text { C }\end{array}$ & - & - & - & 0.375 & $\begin{array}{c}3 \\
(1.5)\end{array}$ & 0.66 & 3 & 2.37 & A5 \\
\hline & (3@3)8-8-T4.0-5\#3-i-2.5-3-9.5 & $\begin{array}{l}\text { A } \\
\text { B } \\
\text { C }\end{array}$ & 10 & $\begin{array}{c}3 \\
(1.5)\end{array}$ & 0.66 & 0.375 & $\begin{array}{c}3 \\
(1.5)\end{array}$ & 0.66 & 3 & 2.37 & A5 \\
\hline \multirow{7}{*}{ Group 12} & 8-5-F4.1-0-i-2.5-7-6 & $\begin{array}{l}A \\
B\end{array}$ & - & - & - & 0.375 & $\begin{array}{c}3 \\
(1.5)\end{array}$ & 0.66 & 2 & 1.58 & A4 \\
\hline & 8-5-F4.1-5\#3-i-2.5-7-6 & $\begin{array}{l}A \\
B\end{array}$ & 10 & $\begin{array}{c}3 \\
(1.5)\end{array}$ & 0.66 & 0.375 & $\begin{array}{c}3 \\
(1.5)\end{array}$ & 0.66 & 2 & 1.58 & A4 \\
\hline & (3@3)8-5-F4.1-0-i-2.5-7-6 & $\begin{array}{l}\mathrm{A} \\
\mathrm{B} \\
\mathrm{C}\end{array}$ & - & - & - & 0.375 & $\begin{array}{c}3 \\
(1.5)\end{array}$ & 0.66 & 3 & 2.37 & A4 \\
\hline & (3@3)8-5-F4.1-5\#3-i-2.5-7-6 & $\begin{array}{l}\text { A } \\
\text { B } \\
\text { C }\end{array}$ & 10 & $\begin{array}{c}3 \\
(1.5)\end{array}$ & 0.66 & 0.375 & $\begin{array}{c}3 \\
(1.5)\end{array}$ & 0.66 & 3 & 2.37 & A5 \\
\hline & (3@5)8-5-F4.1-0-i-2.5-7-6 & $\begin{array}{l}\mathrm{A} \\
\mathrm{B} \\
\mathrm{C}\end{array}$ & - & - & - & 0.375 & $\begin{array}{c}3 \\
(1.5)\end{array}$ & 0.66 & 3 & 2.37 & A5 \\
\hline & (3@5)8-5-F4.1-5\#3-i-2.5-7-6 & $\begin{array}{l}\text { A } \\
\text { B } \\
\text { C }\end{array}$ & 10 & $\begin{array}{c}3 \\
(1.5)\end{array}$ & 0.66 & 0.375 & $\begin{array}{c}3 \\
(1.5)\end{array}$ & 0.66 & 3 & 2.37 & A5 \\
\hline & (3@7)8-5-F4.1-0-i-2.5-7-6 & $\begin{array}{l}\text { A } \\
B \\
C\end{array}$ & - & - & - & 0.375 & $\begin{array}{c}3 \\
(1.5)\end{array}$ & 0.66 & 3 & 2.37 & A8 \\
\hline
\end{tabular}

${ }^{*}$ Value in parenthesis is the spacing between the first hoop and the center of the headed bar 
Table A.3 Cont. Comprehensive test results and data for beam-column joint specimens

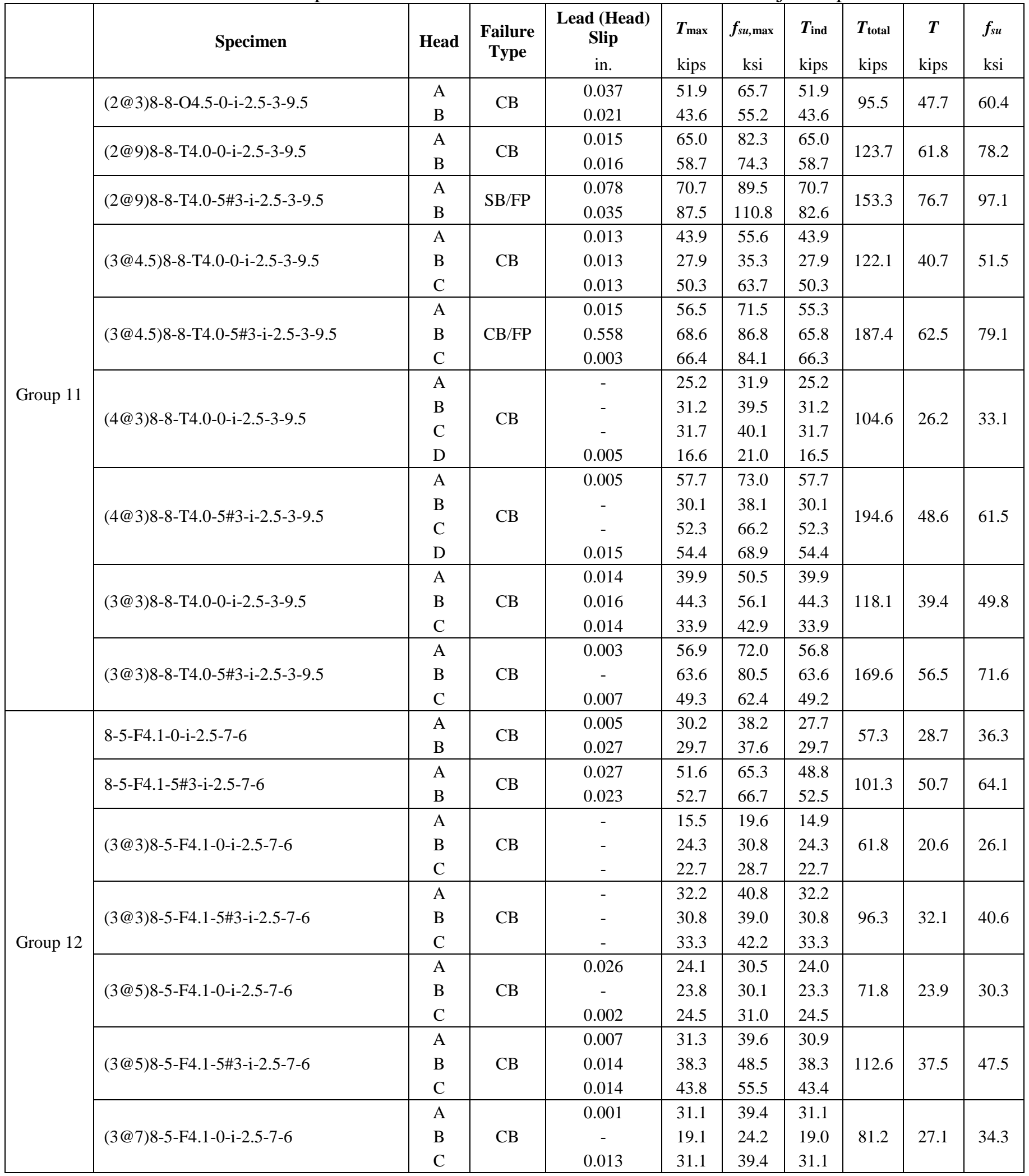


Table A.3 Cont. Comprehensive test results and data for beam-column joint specimens

\begin{tabular}{|c|c|c|c|c|c|c|c|c|c|c|}
\hline & Specimen & Head & $\begin{array}{l}c_{o} \\
\text { in. }\end{array}$ & $A_{b r g}$ & $\begin{array}{l}\ell e h \\
\text { in. }\end{array}$ & $\begin{array}{c}\ell \text { eh,avg } \\
\text { in. }\end{array}$ & $\begin{array}{l}\boldsymbol{f}_{\boldsymbol{c m}} \\
\mathrm{psi}\end{array}$ & $\begin{array}{l}\text { Age } \\
\text { days }\end{array}$ & $\begin{array}{l}\boldsymbol{d}_{\boldsymbol{b}} \\
\text { in. }\end{array}$ & $\begin{array}{l}\boldsymbol{A}_{\boldsymbol{b}} \\
\text { in. }^{2}\end{array}$ \\
\hline \multirow{7}{*}{ Group 12} & (3@7)8-5-F4.1-5\#3-i-2.5-7-6 & $\begin{array}{l}\text { A } \\
\text { B } \\
\text { C }\end{array}$ & 2.0 & $4.1 A_{b}$ & $\begin{array}{l}6.00 \\
6.19 \\
6.13\end{array}$ & 6.10 & 4940 & 15 & 1 & 0.79 \\
\hline & 8-5-F9.1-0-i-2.5-7-6 & $\begin{array}{l}\text { A } \\
B\end{array}$ & 2.0 & $9.1 A_{b}$ & $\begin{array}{l}6.13 \\
6.13\end{array}$ & 6.13 & 4940 & 15 & 1 & 0.79 \\
\hline & 8-5-F9.1-5\#3-i-2.5-7-6 & $\begin{array}{l}\text { A } \\
B\end{array}$ & 2.0 & $9.1 A_{b}$ & $\begin{array}{l}6.19 \\
6.13\end{array}$ & 6.16 & 4940 & 15 & 1 & 0.79 \\
\hline & (3@5.5)8-5-F9.1-0-i-2.5-7-6 & $\begin{array}{l}\text { A } \\
\text { B } \\
\text { C }\end{array}$ & 2.0 & $9.1 A_{b}$ & $\begin{array}{l}6.25 \\
6.13 \\
6.25\end{array}$ & 6.21 & 5160 & 16 & 1 & 0.79 \\
\hline & (3@5.5)8-5-F9.1-5\#3-i-2.5-7-6 & $\begin{array}{l}\mathrm{A} \\
\mathrm{B} \\
\mathrm{C}\end{array}$ & 2.0 & $9.1 A_{b}$ & $\begin{array}{l}6.13 \\
6.25 \\
6.38 \\
\end{array}$ & 6.25 & 5160 & 16 & 1 & 0.79 \\
\hline & (4@3.7)8-5-T9.5-0-i-2.5-6.5-6 & $\begin{array}{l}\text { A } \\
B \\
C \\
D\end{array}$ & 1.4 & $9.5 A_{b}$ & $\begin{array}{l}6.19 \\
6.13 \\
6.19 \\
6.00\end{array}$ & 6.13 & 5160 & 16 & 1 & 0.79 \\
\hline & (4@3.7)8-5-F9.1-5\#3-i-2.5-7-6 & $\begin{array}{l}\text { A } \\
B \\
C \\
D\end{array}$ & 2.0 & $9.1 A_{b}$ & $\begin{array}{l}6.00 \\
6.00 \\
6.00 \\
6.13\end{array}$ & 6.03 & 5160 & 16 & 1 & 0.79 \\
\hline \multirow{11}{*}{ Group 13} & $5-5-F 4.0-0-i-2.5-5-4$ & $\begin{array}{l}\text { A } \\
B\end{array}$ & 2.2 & $4.0 A_{b}$ & $\begin{array}{l}4.00 \\
4.13\end{array}$ & 4.06 & 4810 & 8 & 0.625 & 0.31 \\
\hline & 5-5-F13.1-0-i-2.5-5-4 & $\begin{array}{l}A \\
B\end{array}$ & 2.2 & $13.1 A_{b}$ & $\begin{array}{l}4.25 \\
4.56\end{array}$ & 4.41 & 4810 & 8 & 0.625 & 0.31 \\
\hline & 5-5-F4.0-2\#3-i-2.5-5-4 & $\begin{array}{l}\text { A } \\
B\end{array}$ & 2.2 & $4.0 A_{b}$ & $\begin{array}{l}3.88 \\
3.75 \\
\end{array}$ & 3.81 & 4810 & 8 & 0.625 & 0.31 \\
\hline & 5-5-F13.1-2\#3-i-2.5-5-4 & $\begin{array}{l}\text { A } \\
B\end{array}$ & 2.2 & $13.1 A_{b}$ & $\begin{array}{l}4.25 \\
3.94\end{array}$ & 4.09 & 4810 & 8 & 0.625 & 0.31 \\
\hline & 5-5-F4.0-5\#3-i-2.5-5-4 & $\begin{array}{l}\text { A } \\
B\end{array}$ & 2.2 & $4.0 A_{b}$ & $\begin{array}{l}3.94 \\
4.38\end{array}$ & 4.16 & 4810 & 8 & 0.625 & 0.31 \\
\hline & 5-5-F13.1-5\#3-i-2.5-5-4 & $\begin{array}{l}\text { A } \\
B\end{array}$ & 2.2 & $13.1 A_{b}$ & $\begin{array}{l}4.13 \\
4.25\end{array}$ & 4.19 & 4690 & 7 & 0.625 & 0.31 \\
\hline & 5-5-F4.0-0-i-2.5-3-6 & $\begin{array}{l}\text { A } \\
B\end{array}$ & 2.2 & $4.0 A_{b}$ & $\begin{array}{l}6.00 \\
6.00\end{array}$ & 6.00 & 4690 & 7 & 0.625 & 0.31 \\
\hline & 5-5-F13.1-0-i-2.5-3-6 & $\begin{array}{l}\text { A } \\
B\end{array}$ & 2.2 & $13.1 A_{b}$ & $\begin{array}{l}6.13 \\
6.31\end{array}$ & 6.22 & 4690 & 7 & 0.625 & 0.31 \\
\hline & 5-5-F4.0-2\#3-i-2.5-3-6 & $\begin{array}{l}\text { A } \\
B\end{array}$ & 2.2 & $4.0 A_{b}$ & $\begin{array}{l}6.00 \\
6.00\end{array}$ & 6.00 & 4690 & 7 & 0.625 & 0.31 \\
\hline & 5-5-F13.1-2\#3-i-2.5-3-6 & $\begin{array}{l}\text { A } \\
B\end{array}$ & 2.2 & $13.1 A_{b}$ & $\begin{array}{l}5.88 \\
6.00\end{array}$ & 5.94 & 4690 & 7 & 0.625 & 0.31 \\
\hline & 5-5-F4.0-5\#3-i-2.5-3-6 & $\begin{array}{l}\text { A } \\
B\end{array}$ & 2.2 & $4.0 A_{b}$ & $\begin{array}{l}6.00 \\
6.13\end{array}$ & 6.06 & 4690 & 7 & 0.625 & 0.31 \\
\hline
\end{tabular}


Table A.3 Cont. Comprehensive test results and data for beam-column joint specimens

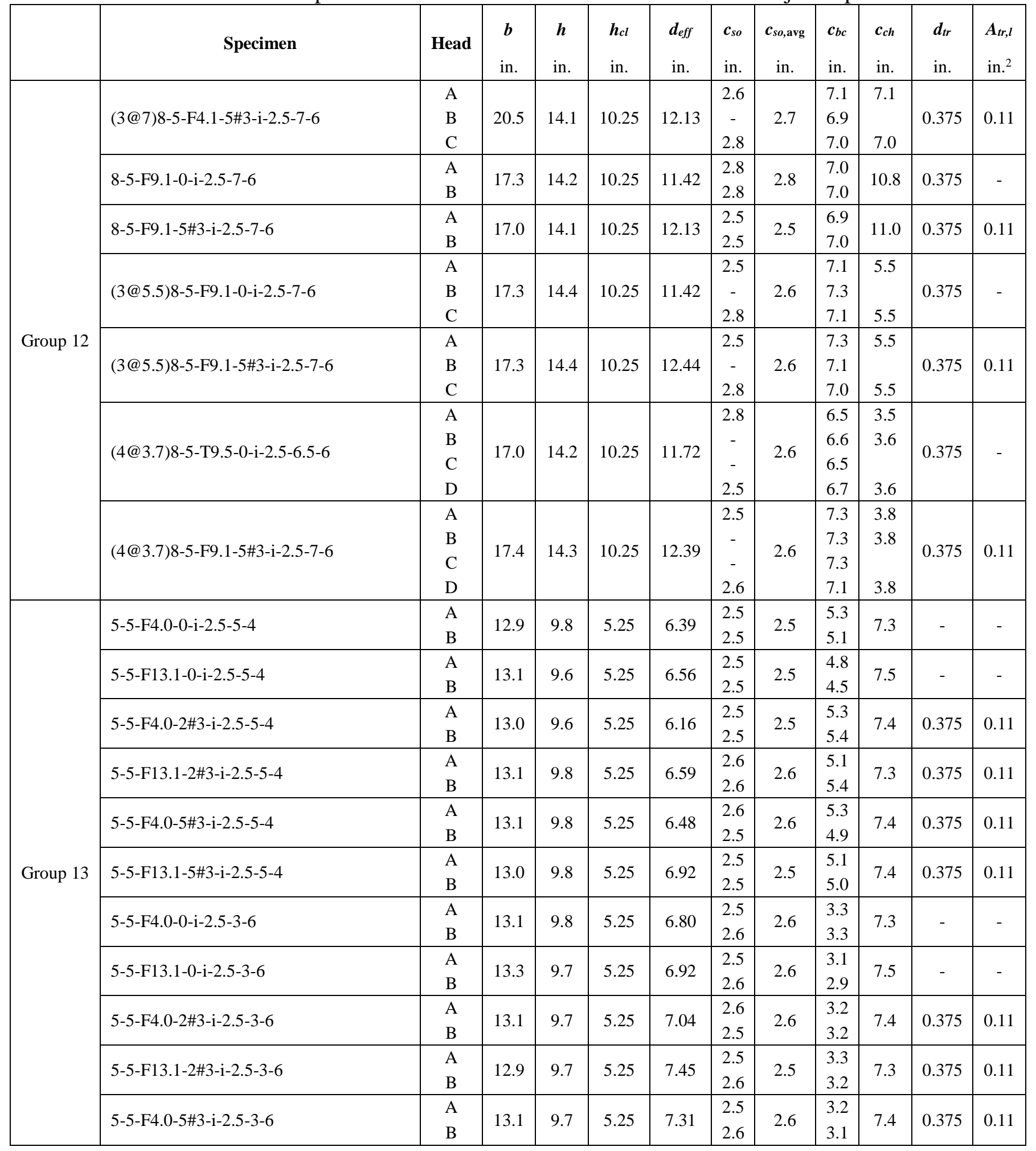


Table A.3 Cont. Comprehensive test results and data for beam-column joint specimens

\begin{tabular}{|c|c|c|c|c|c|c|c|c|c|c|c|}
\hline & Specimen & Head & $N$ & $\begin{array}{l}s_{t r}{ }^{*} \\
\text { in. }\end{array}$ & $\begin{array}{l}\boldsymbol{A}_{\boldsymbol{t t}} \\
\text { in. }^{2}\end{array}$ & $\begin{array}{l}\boldsymbol{d}_{\text {tro }} \\
\text { in. }\end{array}$ & $\begin{array}{l}\text { stro }^{*} \\
\text { in. }\end{array}$ & $\begin{array}{l}\boldsymbol{A}_{\boldsymbol{a} \boldsymbol{b}} \\
\text { in. }^{2}\end{array}$ & $n$ & $\begin{array}{l}\boldsymbol{A}_{\boldsymbol{h s}} \\
\text { in. }^{2}\end{array}$ & $\begin{array}{l}\text { Long. } \\
\text { Reinf. } \\
\text { Layout }\end{array}$ \\
\hline \multirow{7}{*}{ Group 12} & (3@7)8-5-F4.1-5\#3-i-2.5-7-6 & $\begin{array}{l}\text { A } \\
\text { B } \\
\text { C }\end{array}$ & 10 & $\begin{array}{c}3 \\
(1.5)\end{array}$ & 0.66 & 0.5 & $\begin{array}{c}4 \\
(2)\end{array}$ & 0.80 & 3 & 2.37 & A8 \\
\hline & 8-5-F9.1-0-i-2.5-7-6 & $\begin{array}{l}\text { A } \\
\text { B }\end{array}$ & - & - & - & 0.375 & $\begin{array}{c}3 \\
(1.5) \\
\end{array}$ & 0.66 & 2 & 1.58 & A4 \\
\hline & 8-5-F9.1-5\#3-i-2.5-7-6 & $\begin{array}{l}\text { A } \\
B\end{array}$ & 10 & $\begin{array}{c}3 \\
(1.5)\end{array}$ & 0.66 & 0.375 & $\begin{array}{c}3 \\
(1.5)\end{array}$ & 0.66 & 2 & 1.58 & A9 \\
\hline & (3@5.5)8-5-F9.1-0-i-2.5-7-6 & $\begin{array}{l}\text { A } \\
B \\
C\end{array}$ & - & - & - & 0.375 & $\begin{array}{c}3 \\
(1.5)\end{array}$ & 0.66 & 3 & 2.37 & A5 \\
\hline & (3@5.5)8-5-F9.1-5\#3-i-2.5-7-6 & $\begin{array}{l}\text { A } \\
\text { B } \\
\text { C }\end{array}$ & 10 & $\begin{array}{c}3 \\
(1.5)\end{array}$ & 0.66 & 0.5 & $\begin{array}{c}4 \\
(2)\end{array}$ & 0.80 & 3 & 2.37 & A5 \\
\hline & (4@3.7)8-5-T9.5-0-i-2.5-6.5-6 & $\begin{array}{l}\mathrm{A} \\
\mathrm{B} \\
\mathrm{C} \\
\mathrm{D}\end{array}$ & - & - & - & 0.375 & $\begin{array}{c}3 \\
(1.5)\end{array}$ & 0.66 & 4 & 3.16 & A5 \\
\hline & (4@3.7)8-5-F9.1-5\#3-i-2.5-7-6 & $\begin{array}{l}\text { A } \\
\text { B } \\
\text { C } \\
\text { D }\end{array}$ & 10 & $\begin{array}{c}3 \\
(1.5)\end{array}$ & 0.66 & 0.5 & $\begin{array}{c}3 \\
(1.5)\end{array}$ & 1.20 & 4 & 3.16 & A5 \\
\hline \multirow{11}{*}{ Group 13} & $5-5-F 4.0-0-i-2.5-5-4$ & $\begin{array}{l}\mathrm{A} \\
\mathrm{B}\end{array}$ & - & - & - & 0.375 & $\begin{array}{c}3.5 \\
(1.75)\end{array}$ & 0.22 & 2 & 0.62 & A4 \\
\hline & 5-5-F13.1-0-i-2.5-5-4 & $\begin{array}{l}\text { A } \\
\text { B }\end{array}$ & - & - & - & 0.375 & $\begin{array}{c}3.5 \\
(1.75)\end{array}$ & 0.22 & 2 & 0.62 & A4 \\
\hline & 5-5-F4.0-2\#3-i-2.5-5-4 & $\begin{array}{l}\text { A } \\
\text { B }\end{array}$ & 4 & $\begin{array}{c}3.5 \\
(2.625)\end{array}$ & 0.22 & 0.375 & $\begin{array}{c}3.5 \\
(1.75)\end{array}$ & 0.22 & 2 & 0.62 & A4 \\
\hline & 5-5-F13.1-2\#3-i-2.5-5-4 & $\begin{array}{l}\text { A } \\
\text { B }\end{array}$ & 4 & $\begin{array}{c}3.5 \\
(2.625)\end{array}$ & 0.22 & 0.375 & $\begin{array}{c}3.5 \\
(1.75)\end{array}$ & 0.22 & 2 & 0.62 & A4 \\
\hline & 5-5-F4.0-5\#3-i-2.5-5-4 & $\begin{array}{l}\mathrm{A} \\
\mathrm{B}\end{array}$ & 10 & $\begin{array}{c}1.75 \\
(0.875)\end{array}$ & 0.66 & 0.375 & $\begin{array}{c}3.5 \\
(1.75) \\
\end{array}$ & 0.22 & 2 & 0.62 & A4 \\
\hline & 5-5-F13.1-5\#3-i-2.5-5-4 & $\begin{array}{l}\mathrm{A} \\
\mathrm{B}\end{array}$ & 10 & $\begin{array}{c}1.75 \\
(0.875)\end{array}$ & 0.66 & 0.375 & $\begin{array}{c}3.5 \\
(1.75) \\
\end{array}$ & 0.22 & 2 & 0.62 & A4 \\
\hline & 5-5-F4.0-0-i-2.5-3-6 & $\begin{array}{l}\text { A } \\
\text { B }\end{array}$ & - & - & - & 0.375 & $\begin{array}{c}3.5 \\
(1.75)\end{array}$ & 0.22 & 2 & 0.62 & A4 \\
\hline & 5-5-F13.1-0-i-2.5-3-6 & $\begin{array}{l}\text { A } \\
\text { B }\end{array}$ & - & - & - & 0.375 & $\begin{array}{c}3.5 \\
(1.75)\end{array}$ & 0.22 & 2 & 0.62 & A4 \\
\hline & 5-5-F4.0-2\#3-i-2.5-3-6 & $\begin{array}{l}\text { A } \\
\text { B }\end{array}$ & 4 & $\begin{array}{c}3.5 \\
(2.625)\end{array}$ & 0.22 & 0.375 & $\begin{array}{c}3.5 \\
(1.75)\end{array}$ & 0.22 & 2 & 0.62 & A4 \\
\hline & 5-5-F13.1-2\#3-i-2.5-3-6 & $\begin{array}{l}\text { A } \\
\text { B }\end{array}$ & 4 & $\begin{array}{c}3.5 \\
(2.625)\end{array}$ & 0.22 & 0.375 & $\begin{array}{c}3.5 \\
(1.75)\end{array}$ & 0.22 & 2 & 0.62 & A4 \\
\hline & 5-5-F4.0-5\#3-i-2.5-3-6 & $\begin{array}{l}\text { A } \\
\text { B }\end{array}$ & 10 & $\begin{array}{c}1.75 \\
(0.875)\end{array}$ & 0.66 & 0.375 & $\begin{array}{c}3.5 \\
(1.75)\end{array}$ & 0.22 & 2 & 0.62 & A4 \\
\hline
\end{tabular}

${ }^{*}$ Value in parenthesis is the spacing between the first hoop and the center of the headed bar 
Table A.3 Cont. Comprehensive test results and data for beam-column joint specimens

\begin{tabular}{|c|c|c|c|c|c|c|c|c|c|c|}
\hline & Specimen & Head & $\begin{array}{c}\text { Failure } \\
\text { Type }\end{array}$ & $\begin{array}{c}\text { Lead (Head) } \\
\text { Slip } \\
\text { in. }\end{array}$ & $\begin{array}{l}\boldsymbol{T}_{\max } \\
\text { kips }\end{array}$ & $\begin{array}{c}\boldsymbol{f}_{\text {su,max }} \\
\text { ksi }\end{array}$ & $\begin{array}{l}\boldsymbol{T}_{\text {ind }} \\
\text { kips }\end{array}$ & $\begin{array}{l}\boldsymbol{T}_{\text {total }} \\
\text { kips }\end{array}$ & $\begin{array}{c}\boldsymbol{T} \\
\text { kips }\end{array}$ & $\begin{array}{l}\boldsymbol{f}_{\text {su }} \\
\mathrm{ksi}\end{array}$ \\
\hline \multirow{7}{*}{ Group 12} & (3@7)8-5-F4.1-5\#3-i-2.5-7-6 & $\begin{array}{l}\text { A } \\
\text { B } \\
\text { C }\end{array}$ & CB & $\begin{array}{l}- \\
- \\
-\end{array}$ & $\begin{array}{l}44.1 \\
35.2 \\
47.5 \\
\end{array}$ & $\begin{array}{l}55.8 \\
44.6 \\
60.1 \\
\end{array}$ & $\begin{array}{l}44.1 \\
35.2 \\
47.5 \\
\end{array}$ & 126.8 & 42.3 & 53.5 \\
\hline & 8-5-F9.1-0-i-2.5-7-6 & $\begin{array}{l}\text { A } \\
\text { B }\end{array}$ & CB & $\begin{array}{c}0.001 \\
-\end{array}$ & $\begin{array}{l}32.4 \\
34.8\end{array}$ & $\begin{array}{l}41.0 \\
44.1\end{array}$ & $\begin{array}{l}32.0 \\
34.8\end{array}$ & 66.8 & 33.4 & 42.3 \\
\hline & 8-5-F9.1-5\#3-i-2.5-7-6 & $\begin{array}{l}\text { A } \\
\text { B }\end{array}$ & CB & $\begin{array}{l}0.017 \\
0.033\end{array}$ & $\begin{array}{l}53.4 \\
54.3\end{array}$ & $\begin{array}{l}67.6 \\
68.7\end{array}$ & $\begin{array}{l}53.4 \\
54.2\end{array}$ & 107.6 & 53.8 & 68.1 \\
\hline & (3@5.5)8-5-F9.1-0-i-2.5-7-6 & $\begin{array}{l}\mathrm{A} \\
\mathrm{B} \\
\mathrm{C} \\
\end{array}$ & CB & $\begin{array}{c}0.014 \\
- \\
0.015 \\
\end{array}$ & $\begin{array}{l}28.6 \\
13.9 \\
26.4 \\
\end{array}$ & $\begin{array}{l}36.2 \\
17.6 \\
33.4 \\
\end{array}$ & $\begin{array}{l}28.6 \\
13.9 \\
26.4 \\
\end{array}$ & 68.9 & 23.0 & 29.1 \\
\hline & (3@5.5)8-5-F9.1-5\#3-i-2.5-7-6 & $\begin{array}{l}\text { A } \\
\text { B } \\
\text { C }\end{array}$ & CB & $\begin{array}{c}0.025 \\
- \\
0.022\end{array}$ & $\begin{array}{l}40.5 \\
46.5 \\
43.0\end{array}$ & $\begin{array}{l}51.3 \\
58.9 \\
54.4\end{array}$ & $\begin{array}{l}39.9 \\
46.5 \\
43.0\end{array}$ & 129.4 & 43.1 & 54.6 \\
\hline & (4@3.7)8-5-T9.5-0-i-2.5-6.5-6 & $\begin{array}{l}\text { A } \\
B \\
C \\
D\end{array}$ & $\mathrm{CB}$ & $\begin{array}{c}0.001 \\
0.016 \\
- \\
0.024\end{array}$ & $\begin{array}{l}25.9 \\
14.6 \\
17.8 \\
28.8\end{array}$ & $\begin{array}{l}32.8 \\
18.5 \\
22.5 \\
36.5\end{array}$ & $\begin{array}{l}25.9 \\
14.6 \\
17.8 \\
28.6\end{array}$ & 86.9 & 21.7 & 27.5 \\
\hline & (4@3.7)8-5-F9.1-5\#3-i-2.5-7-6 & $\begin{array}{l}\text { A } \\
B \\
C \\
D\end{array}$ & CB & $\begin{array}{c}- \\
- \\
- \\
0.023\end{array}$ & $\begin{array}{l}39.5 \\
31.5 \\
20.4 \\
35.1 \\
\end{array}$ & $\begin{array}{l}50.0 \\
39.9 \\
25.8 \\
44.4 \\
\end{array}$ & $\begin{array}{l}39.5 \\
31.5 \\
20.4 \\
35.1 \\
\end{array}$ & 126.5 & 31.6 & 40.0 \\
\hline \multirow{11}{*}{ Group 13} & $5-5-F 4.0-0-i-2.5-5-4$ & $\begin{array}{l}\mathrm{A} \\
\mathrm{B}\end{array}$ & CB & $\begin{array}{l}- \\
-\end{array}$ & $\begin{array}{l}25.9 \\
23.1 \\
\end{array}$ & $\begin{array}{l}83.5 \\
74.5 \\
\end{array}$ & $\begin{array}{l}25.9 \\
23.1 \\
\end{array}$ & 49.1 & 24.5 & 79.0 \\
\hline & 5-5-F13.1-0-i-2.5-5-4 & $\begin{array}{l}\mathrm{A} \\
\mathrm{B}\end{array}$ & CB & - & $\begin{array}{l}26.6 \\
30.2 \\
\end{array}$ & $\begin{array}{l}85.8 \\
97.4 \\
\end{array}$ & $\begin{array}{l}26.5 \\
29.9\end{array}$ & 56.4 & 28.2 & 91.0 \\
\hline & 5-5-F4.0-2\#3-i-2.5-5-4 & $\begin{array}{l}\text { A } \\
\text { B }\end{array}$ & CB & $\begin{array}{l}- \\
-\end{array}$ & $\begin{array}{l}20.1 \\
19.2\end{array}$ & $\begin{array}{l}64.8 \\
61.9\end{array}$ & $\begin{array}{l}20.1 \\
19.2\end{array}$ & 39.3 & 19.7 & 63.5 \\
\hline & 5-5-F13.1-2\#3-i-2.5-5-4 & $\begin{array}{l}\mathrm{A} \\
\mathrm{B}\end{array}$ & CB & - & $\begin{array}{l}28.6 \\
30.1 \\
\end{array}$ & $\begin{array}{l}92.3 \\
97.1 \\
\end{array}$ & $\begin{array}{l}27.9 \\
29.8 \\
\end{array}$ & 57.7 & 28.9 & 93.2 \\
\hline & 5-5-F4.0-5\#3-i-2.5-5-4 & $\begin{array}{l}\mathrm{A} \\
\mathrm{B}\end{array}$ & CB & $\begin{array}{l}- \\
-\end{array}$ & $\begin{array}{l}27.0 \\
26.1^{\ddagger} \\
\end{array}$ & $\begin{array}{l}87.1 \\
84.2^{\ddagger} \\
\end{array}$ & $\begin{array}{l}27.0 \\
26.0 \\
\end{array}$ & 53.0 & 26.5 & 85.5 \\
\hline & 5-5-F13.1-5\#3-i-2.5-5-4 & $\begin{array}{l}\mathrm{A} \\
\mathrm{B}\end{array}$ & CB & - & $\begin{array}{l}35.5 \\
34.8 \\
\end{array}$ & $\begin{array}{l}114.5 \\
112.3 \\
\end{array}$ & $\begin{array}{l}35.5 \\
34.8\end{array}$ & 70.4 & 35.2 & 113.5 \\
\hline & $5-5-F 4.0-0-i-2.5-3-6$ & $\begin{array}{l}\mathrm{A} \\
\mathrm{B}\end{array}$ & SB & - & $\begin{array}{l}34.6^{\ddagger} \\
33.0 \\
\end{array}$ & $\begin{array}{l}111.6^{\ddagger} \\
106.5\end{array}$ & $\begin{array}{l}32.9 \\
32.6 \\
\end{array}$ & 65.5 & 32.7 & 105.5 \\
\hline & 5-5-F13.1-0-i-2.5-3-6 & $\begin{array}{l}\mathrm{A} \\
\mathrm{B}\end{array}$ & $\mathrm{SB} / \mathrm{FP}$ & - & $\begin{array}{l}33.2^{\ddagger} \\
37.6 \\
\end{array}$ & $\begin{array}{l}107.1^{\ddagger} \\
121.3 \\
\end{array}$ & $\begin{array}{l}33.1 \\
37.5 \\
\end{array}$ & 70.6 & 35.3 & 113.9 \\
\hline & 5-5-F4.0-2\#3-i-2.5-3-6 & $\begin{array}{l}\mathrm{A} \\
\mathrm{B}\end{array}$ & $\mathrm{SB} / \mathrm{FP}$ & - & $\begin{array}{l}40.0 \\
40.3 \\
\end{array}$ & $\begin{array}{l}129.0 \\
130.0 \\
\end{array}$ & $\begin{array}{l}35.5 \\
40.3 \\
\end{array}$ & 75.7 & 37.9 & 122.3 \\
\hline & 5-5-F13.1-2\#3-i-2.5-3-6 & $\begin{array}{l}\mathrm{A} \\
\mathrm{B}\end{array}$ & $\mathrm{SB} / \mathrm{FP}$ & $\begin{array}{l}- \\
-\end{array}$ & $\begin{array}{l}46.3^{\ddagger} \\
46.6 \\
\end{array}$ & $\begin{array}{l}149.4^{\ddagger} \\
150.3 \\
\end{array}$ & $\begin{array}{l}46.3 \\
46.5 \\
\end{array}$ & 92.8 & 46.4 & 149.7 \\
\hline & 5-5-F4.0-5\#3-i-2.5-3-6 & $\begin{array}{l}\text { A } \\
\text { B }\end{array}$ & $\mathrm{SB} / \mathrm{FP}$ & $\begin{array}{l}- \\
-\end{array}$ & $\begin{array}{l}42.4 \\
44.6\end{array}$ & $\begin{array}{l}136.8 \\
143.9\end{array}$ & $\begin{array}{l}42.4 \\
44.6\end{array}$ & 86.9 & 43.5 & 140.3 \\
\hline
\end{tabular}

${ }^{\ddagger}$ No anchorage failure on the bar 
Table A.3 Cont. Comprehensive test results and data for beam-column joint specimens

\begin{tabular}{|c|c|c|c|c|c|c|c|c|c|c|}
\hline & Specimen & Head & $\begin{array}{l}\boldsymbol{c}_{\boldsymbol{o}} \\
\text { in. }\end{array}$ & $A_{b r g}$ & $\begin{array}{l}\ell e h \\
\text { in. }\end{array}$ & $\begin{array}{c}\ell \text { eh,avg } \\
\text { in. }\end{array}$ & $\begin{array}{l}f_{c m} \\
\text { psi }\end{array}$ & $\begin{array}{l}\text { Age } \\
\text { days }\end{array}$ & $\begin{array}{l}\boldsymbol{d}_{\boldsymbol{b}} \\
\text { in. }\end{array}$ & $\begin{array}{l}\boldsymbol{A}_{\boldsymbol{b}} \\
\text { in. }^{2}\end{array}$ \\
\hline \multirow{14}{*}{ Group 14} & 5-12-F4.0-0-i-2.5-5-4 & $\begin{array}{l}\text { A } \\
\text { B }\end{array}$ & 2.2 & $4.0 A_{b}$ & $\begin{array}{l}4.13 \\
4.00 \\
\end{array}$ & 4.06 & 11030 & 35 & 0.625 & 0.31 \\
\hline & 5-12-F13.1-0-i-2.5-5-4 & $\begin{array}{l}\text { A } \\
B\end{array}$ & 2.2 & $13.1 A_{b}$ & $\begin{array}{l}4.13 \\
4.13\end{array}$ & 4.13 & 11030 & 35 & 0.625 & 0.31 \\
\hline & 5-12-F4.0-2\#3-i-2.5-5-4 & $\begin{array}{l}\text { A } \\
B\end{array}$ & 2.2 & $4.0 A_{b}$ & $\begin{array}{l}4.13 \\
4.13\end{array}$ & 4.13 & 11030 & 35 & 0.625 & 0.31 \\
\hline & 5-12-F13.1-2\#3-i-2.5-5-4 & $\begin{array}{l}\text { A } \\
B\end{array}$ & 2.2 & $13.1 A_{b}$ & $\begin{array}{l}4.06 \\
4.13\end{array}$ & 4.09 & 11030 & 35 & 0.625 & 0.31 \\
\hline & 5-12-F4.0-5\#3-i-2.5-5-4 & $\begin{array}{l}\mathrm{A} \\
\mathrm{B}\end{array}$ & 2.2 & $4.0 A_{b}$ & $\begin{array}{l}4.19 \\
4.25 \\
\end{array}$ & 4.22 & 11030 & 35 & 0.625 & 0.31 \\
\hline & 5-12-F13.1-5\#3-i-2.5-5-4 & $\begin{array}{l}\text { A } \\
B\end{array}$ & 2.2 & $13.1 A_{b}$ & $\begin{array}{l}4.13 \\
4.13\end{array}$ & 4.13 & 11030 & 35 & 0.625 & 0.31 \\
\hline & 5-12-F4.0-0-i-2.5-3-6 & $\begin{array}{l}\text { A } \\
B\end{array}$ & 2.2 & $4.0 A_{b}$ & $\begin{array}{l}6.00 \\
6.00\end{array}$ & 6.00 & 11030 & 36 & 0.625 & 0.31 \\
\hline & 5-12-F13.1-0-i-2.5-3-6 & $\begin{array}{l}\mathrm{A} \\
\mathrm{B}\end{array}$ & 2.2 & $13.1 A_{b}$ & $\begin{array}{l}6.00 \\
6.06 \\
\end{array}$ & 6.03 & 11030 & 36 & 0.625 & 0.31 \\
\hline & (3@5.9)5-12-F4.0-0-i-2.5-4-5 & $\begin{array}{l}\text { A } \\
B \\
C\end{array}$ & 2.2 & $4.0 A_{b}$ & $\begin{array}{l}5.06 \\
5.06 \\
5.00 \\
\end{array}$ & 5.04 & 11030 & 36 & 0.625 & 0.31 \\
\hline & (3@5.9)5-12-F4.0-2\#3-i-2.5-4-5 & $\begin{array}{l}\text { A } \\
\text { B } \\
\text { C }\end{array}$ & 2.2 & $4.0 A_{b}$ & $\begin{array}{l}5.13 \\
5.13 \\
5.19 \\
\end{array}$ & 5.15 & 11030 & 36 & 0.625 & 0.31 \\
\hline & (3@5.9)5-12-F4.0-5\#3-i-2.5-4-5 & $\begin{array}{l}\text { A } \\
B \\
C \\
\end{array}$ & 2.2 & $4.0 A_{b}$ & $\begin{array}{l}5.19 \\
4.88 \\
5.00 \\
\end{array}$ & 5.02 & 11030 & 36 & 0.625 & 0.31 \\
\hline & (4@3.9)5-12-F4.0-0-i-2.5-4-5 & $\begin{array}{l}A \\
B \\
C \\
D \\
\end{array}$ & 2.2 & $4.0 A_{b}$ & $\begin{array}{l}5.19 \\
5.13 \\
5.25 \\
5.19 \\
\end{array}$ & 5.19 & 11030 & 39 & 0.625 & 0.31 \\
\hline & (4@3.9)5-12-F4.0-2\#3-i-2.5-4-5 & $\begin{array}{l}\text { A } \\
B \\
C \\
D\end{array}$ & 2.2 & $4.0 A_{b}$ & $\begin{array}{l}5.00 \\
5.00 \\
5.13 \\
5.00 \\
\end{array}$ & 5.03 & 11030 & 39 & 0.625 & 0.31 \\
\hline & (4@3.9)5-12-F4.0-5\#3-i-2.5-4-5 & $\begin{array}{l}\text { A } \\
\text { B } \\
\text { C } \\
\text { D }\end{array}$ & 2.2 & $4.0 A_{b}$ & $\begin{array}{l}5.25 \\
5.13 \\
5.19 \\
5.19 \\
\end{array}$ & 5.19 & 11030 & 39 & 0.625 & 0.31 \\
\hline \multirow{3}{*}{ Group 15} & 11-5a-F3.8-0-i-2.5-3-17 & $\begin{array}{l}\mathrm{A} \\
\mathrm{B}\end{array}$ & 2.0 & $3.8 A_{b}$ & $\begin{array}{l}16.38 \\
16.75 \\
\end{array}$ & 16.56 & 4050 & 36 & 1.41 & 1.56 \\
\hline & 11-5a-F3.8-2\#3-i-2.5-3-17 & $\begin{array}{l}\text { A } \\
\text { B }\end{array}$ & 2.0 & $3.8 A_{b}$ & $\begin{array}{l}17.44 \\
17.44 \\
\end{array}$ & 17.44 & 4050 & 36 & 1.41 & 1.56 \\
\hline & $11-5 a-F 3.8-6 \# 3-i-2.5-3-17$ & $\begin{array}{l}\text { A } \\
B\end{array}$ & 2.0 & $3.8 A_{b}$ & $\begin{array}{l}16.75 \\
16.69\end{array}$ & 16.72 & 4050 & 36 & 1.41 & 1.56 \\
\hline
\end{tabular}


Table A.3 Cont. Comprehensive test results and data for beam-column joint specimens

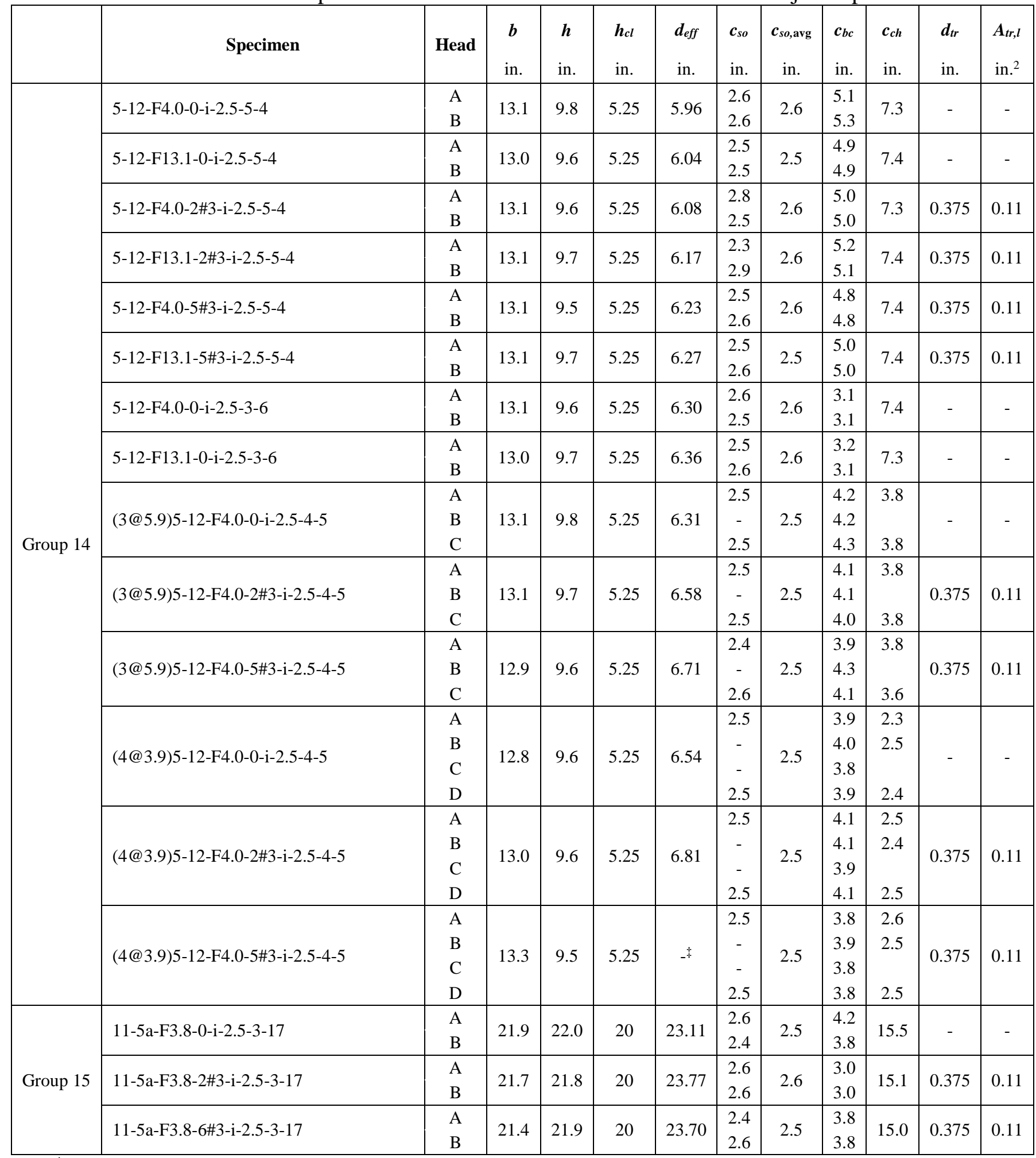

\footnotetext{
${ }^{\ddagger} d_{\text {eff }}$ was not calculated for specimen with bar yielding
} 
Table A.3 Cont. Comprehensive test results and data for beam-column joint specimens

\begin{tabular}{|c|c|c|c|c|c|c|c|c|c|c|c|}
\hline & Specimen & Head & $N$ & $\begin{array}{l}\boldsymbol{s}_{t r}{ }^{*} \\
\text { in. }\end{array}$ & $\begin{array}{l}\boldsymbol{A}_{\boldsymbol{t t}} \\
\text { in. }\end{array}$ & $\begin{array}{l}\boldsymbol{d}_{\text {tro }} \\
\text { in. }\end{array}$ & $\begin{array}{c}\boldsymbol{S}_{\text {tro }}{ }^{*} \\
\text { in. }\end{array}$ & $\begin{array}{l}A_{a b} \\
\text { in. }^{2}\end{array}$ & $n$ & $\begin{array}{l}A_{h s} \\
\text { in. }^{2}\end{array}$ & $\begin{array}{l}\text { Long. } \\
\text { Reinf. } \\
\text { Layout }\end{array}$ \\
\hline \multirow{14}{*}{ Group 14} & 5-12-F4.0-0-i-2.5-5-4 & $\begin{array}{l}\mathrm{A} \\
\mathrm{B}\end{array}$ & - & - & - & 0.375 & $\begin{array}{c}3.5 \\
(1.75)\end{array}$ & 0.22 & 2 & 0.62 & A4 \\
\hline & 5-12-F13.1-0-i-2.5-5-4 & $\begin{array}{l}\mathrm{A} \\
\mathrm{B}\end{array}$ & - & - & - & 0.375 & $\begin{array}{c}3.5 \\
(1.75)\end{array}$ & 0.22 & 2 & 0.62 & A4 \\
\hline & 5-12-F4.0-2\#3-i-2.5-5-4 & $\begin{array}{l}\mathrm{A} \\
\mathrm{B}\end{array}$ & 4 & $\begin{array}{c}3.5 \\
(2.625) \\
\end{array}$ & 0.22 & 0.375 & $\begin{array}{c}3.5 \\
(1.75) \\
\end{array}$ & 0.22 & 2 & 0.62 & A4 \\
\hline & 5-12-F13.1-2\#3-i-2.5-5-4 & $\begin{array}{l}\mathrm{A} \\
\mathrm{B}\end{array}$ & 4 & $\begin{array}{c}3.5 \\
(2.625) \\
\end{array}$ & 0.22 & 0.375 & $\begin{array}{r}3.5 \\
(1.75) \\
\end{array}$ & 0.22 & 2 & 0.62 & A4 \\
\hline & 5-12-F4.0-5\#3-i-2.5-5-4 & $\begin{array}{l}\mathrm{A} \\
\mathrm{B}\end{array}$ & 10 & $\begin{array}{c}1.75 \\
(0.875) \\
\end{array}$ & 0.66 & 0.375 & $\begin{array}{c}3.5 \\
(1.75) \\
\end{array}$ & 0.22 & 2 & 0.62 & A4 \\
\hline & 5-12-F13.1-5\#3-i-2.5-5-4 & $\begin{array}{l}\mathrm{A} \\
\mathrm{B}\end{array}$ & 10 & $\begin{array}{c}1.75 \\
(0.875) \\
\end{array}$ & 0.66 & 0.375 & $\begin{array}{c}3.5 \\
(1.75) \\
\end{array}$ & 0.22 & 2 & 0.62 & A4 \\
\hline & 5-12-F4.0-0-i-2.5-3-6 & $\begin{array}{l}\mathrm{A} \\
\mathrm{B}\end{array}$ & - & - & - & 0.375 & $\begin{array}{c}3.5 \\
(1.75) \\
\end{array}$ & 0.22 & 2 & 0.62 & A4 \\
\hline & 5-12-F13.1-0-i-2.5-3-6 & $\begin{array}{l}\text { A } \\
\mathrm{B}\end{array}$ & - & - & - & 0.375 & $\begin{array}{c}3.5 \\
(1.75) \\
\end{array}$ & 0.22 & 2 & 0.62 & A4 \\
\hline & (3@5.9)5-12-F4.0-0-i-2.5-4-5 & $\begin{array}{l}\text { A } \\
B \\
C\end{array}$ & - & - & - & 0.375 & $\begin{array}{c}3.5 \\
(1.75)\end{array}$ & 0.22 & 3 & 0.93 & A5 \\
\hline & (3@5.9)5-12-F4.0-2\#3-i-2.5-4-5 & $\begin{array}{l}\text { A } \\
B \\
C\end{array}$ & 4 & $\begin{array}{c}3.5 \\
(2.625)\end{array}$ & 0.22 & 0.375 & $\begin{array}{c}3.5 \\
(1.75)\end{array}$ & 0.22 & 3 & 0.93 & A5 \\
\hline & (3@5.9)5-12-F4.0-5\#3-i-2.5-4-5 & $\begin{array}{l}\mathrm{A} \\
\mathrm{B} \\
\mathrm{C} \\
\end{array}$ & 10 & $\begin{array}{c}1.75 \\
(0.875)\end{array}$ & 0.66 & 0.375 & $\begin{array}{c}3.5 \\
(1.75)\end{array}$ & 0.22 & 3 & 0.93 & A5 \\
\hline & (4@3.9)5-12-F4.0-0-i-2.5-4-5 & $\begin{array}{l}\mathrm{A} \\
\mathrm{B} \\
\mathrm{C} \\
\mathrm{D}\end{array}$ & - & - & - & 0.375 & $\begin{array}{c}3.5 \\
(1.75)\end{array}$ & 0.22 & 4 & 1.24 & A5 \\
\hline & (4@3.9)5-12-F4.0-2\#3-i-2.5-4-5 & $\begin{array}{l}\mathrm{A} \\
\mathrm{B} \\
\mathrm{C} \\
\mathrm{D}\end{array}$ & 4 & $\begin{array}{c}3.5 \\
(2.625)\end{array}$ & 0.22 & 0.375 & $\begin{array}{c}3.5 \\
(1.75)\end{array}$ & 0.22 & 4 & 1.24 & A5 \\
\hline & (4@3.9)5-12-F4.0-5\#3-i-2.5-4-5 & $\begin{array}{l}\text { A } \\
\text { B } \\
\text { C } \\
\text { D }\end{array}$ & 10 & $\begin{array}{c}1.75 \\
(0.875)\end{array}$ & 0.22 & 0.375 & $\begin{array}{c}3.5 \\
(1.75)\end{array}$ & 0.18 & 4 & 1.24 & A5 \\
\hline \multirow{3}{*}{ Group 15} & 11-5a-F3.8-0-i-2.5-3-17 & $\begin{array}{l}\text { A } \\
B\end{array}$ & - & - & - & 0.5 & $\begin{array}{c}4 \\
(2) \\
\end{array}$ & 1.20 & 2 & 3.12 & A10 \\
\hline & 11-5a-F3.8-2\#3-i-2.5-3-17 & $\begin{array}{l}\text { A } \\
\text { B }\end{array}$ & 4 & $\begin{array}{c}8 \\
(6) \\
\end{array}$ & 0.22 & 0.5 & $\begin{array}{c}4 \\
(2) \\
\end{array}$ & 1.20 & 2 & 3.12 & A10 \\
\hline & 11-5a-F3.8-6\#3-i-2.5-3-17 & $\begin{array}{l}\mathrm{A} \\
\mathrm{B} \\
\end{array}$ & 12 & $\begin{array}{c}4 \\
(2) \\
\end{array}$ & 0.66 & 0.5 & $\begin{array}{c}4 \\
(2) \\
\end{array}$ & 1.20 & 2 & 3.12 & A10 \\
\hline
\end{tabular}

${ }^{*}$ Value in parenthesis is the spacing between the first hoop and the center of the headed bar 
Table A.3 Cont. Comprehensive test results and data for beam-column joint specimens

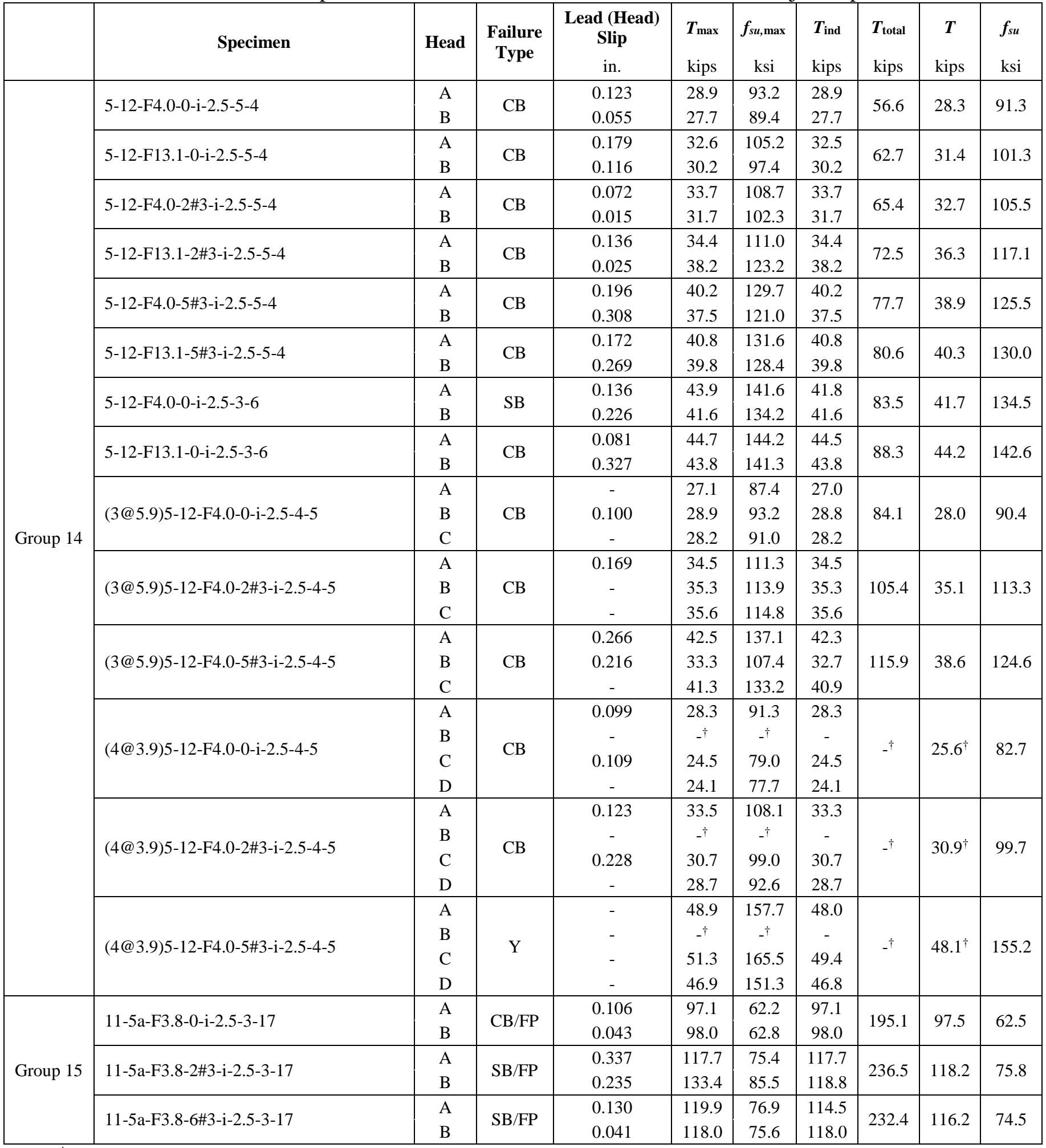

\footnotetext{
${ }^{\dagger}$ Load on headed bar B was not recorded due to a malfunction of load cell; $T$ taken as the average load of the other three bars.
} 
Table A.3 Cont. Comprehensive test results and data for beam-column joint specimens

\begin{tabular}{|c|c|c|c|c|c|c|c|c|c|c|}
\hline & Specimen & Head & $\begin{array}{l}\boldsymbol{c}_{\boldsymbol{o}} \\
\text { in. }\end{array}$ & $A_{b r g}$ & $\begin{array}{l}\ell e h \\
\text { in. }\end{array}$ & $\begin{array}{c}\ell \text { eh,avg } \\
\text { in. }\end{array}$ & $\begin{array}{l}\boldsymbol{f}_{\boldsymbol{c m}} \\
\mathrm{psi}\end{array}$ & $\begin{array}{l}\text { Age } \\
\text { days }\end{array}$ & $\begin{array}{l}\boldsymbol{d}_{\boldsymbol{b}} \\
\text { in. }\end{array}$ & $\begin{array}{l}A_{b} \\
\text { in. }^{2}\end{array}$ \\
\hline \multirow{5}{*}{ Group 15} & 11-5a-F3.8-0-i-2.5-3-12 & $\begin{array}{l}\text { A } \\
B\end{array}$ & 2.0 & $3.8 A_{b}$ & $\begin{array}{l}12.19 \\
11.81\end{array}$ & 12.00 & 3960 & 35 & 1.41 & 1.56 \\
\hline & 11-5a-F3.8-2\#3-i-2.5-3-12 & $\begin{array}{l}\text { A } \\
B\end{array}$ & 2.0 & $3.8 A_{b}$ & $\begin{array}{l}11.81 \\
12.19\end{array}$ & 12.00 & 3960 & 35 & 1.41 & 1.56 \\
\hline & 11-5a-F3.8-6\#3-i-2.5-3-12 & $\begin{array}{l}\text { A } \\
B\end{array}$ & 2.0 & $3.8 A_{b}$ & $\begin{array}{l}12.13 \\
12.06\end{array}$ & 12.09 & 3960 & 35 & 1.41 & 1.56 \\
\hline & 11-5a-F8.6-0-i-2.5-3-12 & $\begin{array}{l}\text { A } \\
\text { B }\end{array}$ & 2.0 & $8.6 A_{b}$ & $\begin{array}{l}12.13 \\
12.13\end{array}$ & 12.13 & 3960 & 35 & 1.41 & 1.56 \\
\hline & 11-5a-F8.6-6\#3-i-2.5-3-12 & $\begin{array}{l}\mathrm{A} \\
\mathrm{B}\end{array}$ & 2.0 & $8.6 A_{b}$ & $\begin{array}{l}12.69 \\
12.44 \\
\end{array}$ & 12.56 & 4050 & 36 & 1.41 & 1.56 \\
\hline \multirow{9}{*}{ Group 16} & 8-8-F4.1-0-i-2.5-3-10-DB & $\begin{array}{l}A \\
B\end{array}$ & 2.0 & $4.1 A_{b}$ & $\begin{array}{l}9.88 \\
9.88\end{array}$ & 9.88 & 7410 & 49 & 1 & 0.79 \\
\hline & 8-8-F9.1-0-i-2.5-3-10-DB & $\begin{array}{l}\text { A } \\
B\end{array}$ & 2.0 & $9.1 A_{b}$ & $\begin{array}{l}9.88 \\
9.75\end{array}$ & 9.81 & 7410 & 49 & 1 & 0.79 \\
\hline & 8-8-F9.1-5\#3-i-2.5-3-10-DB & $\begin{array}{l}\text { A } \\
B\end{array}$ & 2.0 & $9.1 A_{b}$ & $\begin{array}{l}9.63 \\
9.63\end{array}$ & 9.63 & 7410 & 49 & 1 & 0.79 \\
\hline & 8-5-F4.1-0-i-2.5-3-10-DB & $\begin{array}{l}\text { A } \\
\mathrm{B}\end{array}$ & 2.0 & $4.1 A_{b}$ & $\begin{array}{l}9.88 \\
9.88 \\
\end{array}$ & 9.88 & 4880 & 19 & 1 & 0.79 \\
\hline & 8-5-F9.1-0-i-2.5-3-10-DB & $\begin{array}{l}\text { A } \\
B\end{array}$ & 2.0 & $9.1 A_{b}$ & $\begin{array}{l}9.63 \\
9.88\end{array}$ & 9.75 & 4880 & 19 & 1 & 0.79 \\
\hline & 8-5-F4.1-3\#4-i-2.5-3-10-DB & $\begin{array}{l}\text { A } \\
B\end{array}$ & 2.0 & $4.1 A_{b}$ & $\begin{array}{l}10.00 \\
10.25\end{array}$ & 10.13 & 4880 & 19 & 1 & 0.79 \\
\hline & 8-5-F9.1-3\#4-i-2.5-3-10-DB & $\begin{array}{l}\text { A } \\
B\end{array}$ & 2.0 & $9.1 A_{b}$ & $\begin{array}{l}9.75 \\
9.75\end{array}$ & 9.75 & 4880 & 20 & 1 & 0.79 \\
\hline & 8-5-F4.1-5\#3-i-2.5-3-10-DB & $\begin{array}{l}\text { A } \\
\mathrm{B}\end{array}$ & 2.0 & $4.1 A_{b}$ & $\begin{array}{l}10.25 \\
10.13 \\
\end{array}$ & 10.19 & 4880 & 20 & 1 & 0.79 \\
\hline & 8-5-F9.1-5\#3-i-2.5-3-10-DB & $\begin{array}{l}\text { A } \\
B\end{array}$ & 2.0 & $9.1 A_{b}$ & $\begin{array}{c}10.00 \\
9.88\end{array}$ & 9.94 & 4880 & 20 & 1 & 0.79 \\
\hline \multirow{6}{*}{ Group 17} & 11-8-F3.8-0-i-2.5-3-14.5 & $\begin{array}{l}\text { A } \\
B\end{array}$ & 2.0 & $3.8 A_{b}$ & $\begin{array}{l}14.50 \\
14.50\end{array}$ & 14.50 & 8660 & 19 & 1.41 & 1.56 \\
\hline & 11-8-F3.8-2\#3-i-2.5-3-14.5 & $\begin{array}{l}\text { A } \\
B\end{array}$ & 2.0 & $3.8 A_{b}$ & $\begin{array}{l}14.63 \\
14.75\end{array}$ & 14.69 & 8660 & 19 & 1.41 & 1.56 \\
\hline & 11-8-F3.8-6\#3-i-2.5-3-14.5 & $\begin{array}{l}\text { A } \\
\text { B }\end{array}$ & 2.0 & $3.8 A_{b}$ & $\begin{array}{l}14.88 \\
14.50 \\
\end{array}$ & 14.69 & 8660 & 19 & 1.41 & 1.56 \\
\hline & (3@5.35)11-8-F3.8-0-i-2.5-3-14.5 & $\begin{array}{l}\mathrm{A} \\
\mathrm{B} \\
\mathrm{C}\end{array}$ & 2.0 & $3.8 A_{b}$ & $\begin{array}{l}14.38 \\
14.75 \\
14.75 \\
\end{array}$ & 14.63 & 8720 & 20 & 1.41 & 1.56 \\
\hline & (3@5.35)11-8-F3.8-2\#3-i-2.5-3-14.5 & $\begin{array}{l}\text { A } \\
B \\
C\end{array}$ & 2.0 & $3.8 A_{b}$ & $\begin{array}{l}14.50 \\
14.63 \\
14.50\end{array}$ & 14.54 & 8720 & 20 & 1.41 & 1.56 \\
\hline & (3@5.35)11-8-F3.8-6\#3-i-2.5-3-14.5 & $\begin{array}{l}\text { A } \\
B \\
C\end{array}$ & 2.0 & $3.8 A_{b}$ & $\begin{array}{l}15.13 \\
14.88 \\
14.75\end{array}$ & 14.92 & 8720 & 20 & 1.41 & 1.56 \\
\hline
\end{tabular}


Table A.3 Cont. Comprehensive test results and data for beam-column joint specimens

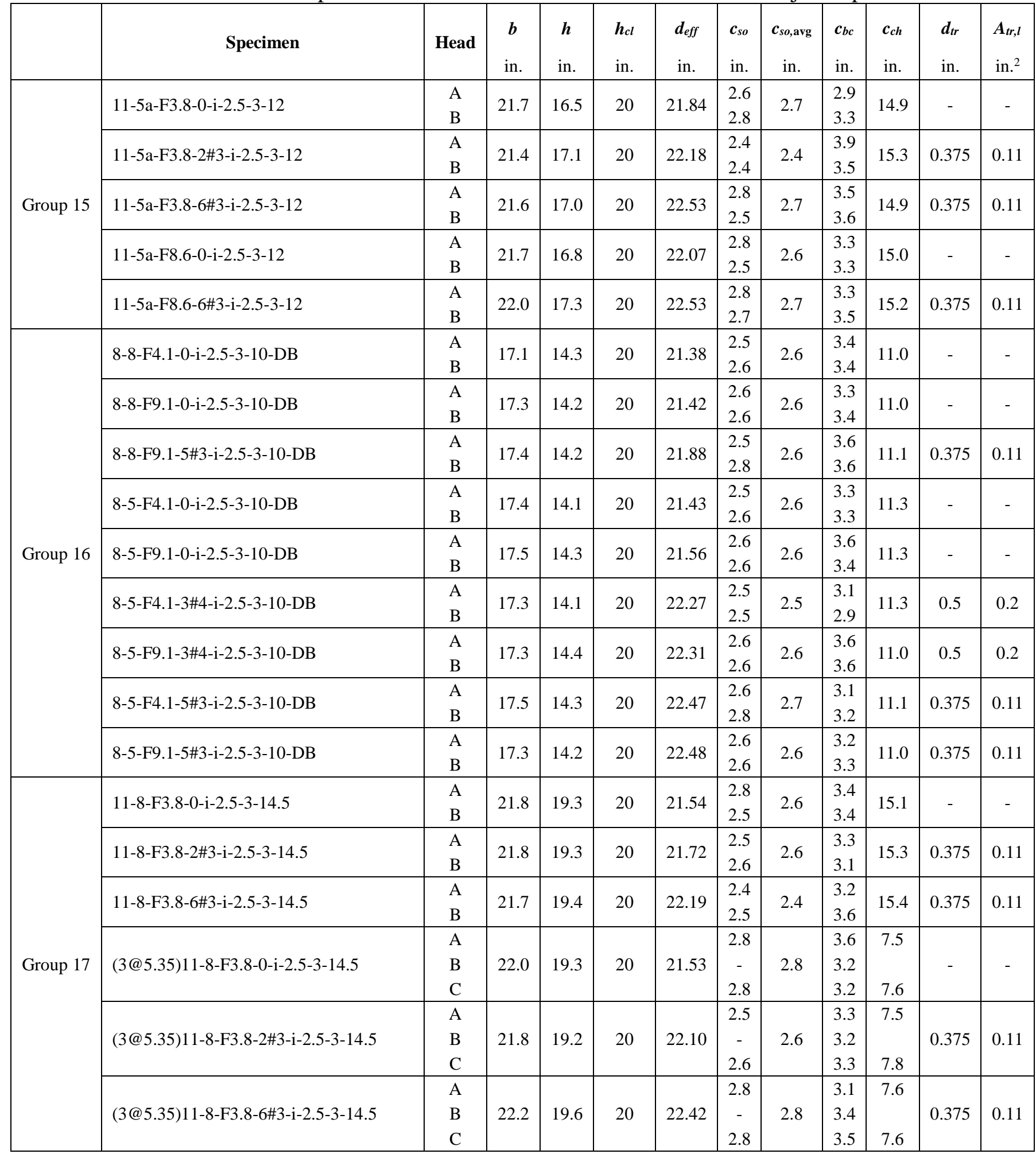


Table A.3 Cont. Comprehensive test results and data for beam-column joint specimens

\begin{tabular}{|c|c|c|c|c|c|c|c|c|c|c|c|}
\hline & Specimen & Head & $N$ & $\begin{array}{l}\boldsymbol{s}_{t r}^{*} \\
\text { in. }\end{array}$ & $\begin{array}{l}\boldsymbol{A}_{t t} \\
\text { in. }^{2}\end{array}$ & $\begin{array}{c}\boldsymbol{d}_{\text {tro }} \\
\text { in. }\end{array}$ & $\begin{array}{l}\text { Stro }^{*} \\
\text { in. }\end{array}$ & $\begin{array}{l}\boldsymbol{A}_{\boldsymbol{a} b} \\
\text { in. }^{2}\end{array}$ & $n$ & $\begin{array}{l}A_{h s} \\
\text { in. }^{2}\end{array}$ & $\begin{array}{l}\text { Long. } \\
\text { Reinf. } \\
\text { Layout }\end{array}$ \\
\hline \multirow{5}{*}{ Group 15} & 11-5a-F3.8-0-i-2.5-3-12 & $\begin{array}{l}\text { A } \\
B\end{array}$ & - & - & - & 0.5 & $\begin{array}{c}5 \\
(2.5)\end{array}$ & 1.20 & 2 & 3.12 & A11 \\
\hline & 11-5a-F3.8-2\#3-i-2.5-3-12 & $\begin{array}{l}\text { A } \\
B\end{array}$ & 4 & $\begin{array}{c}8 \\
(6) \\
\end{array}$ & 0.22 & 0.5 & $\begin{array}{c}5 \\
(2.5) \\
\end{array}$ & 1.20 & 2 & 3.12 & A11 \\
\hline & 11-5a-F3.8-6\#3-i-2.5-3-12 & $\begin{array}{l}\text { A } \\
B\end{array}$ & 12 & $\begin{array}{c}4 \\
(2) \\
\end{array}$ & 0.66 & 0.5 & $\begin{array}{c}5 \\
(2.5) \\
\end{array}$ & 1.20 & 2 & 3.12 & A11 \\
\hline & 11-5a- F8.6-0-i-2.5-3-12 & $\begin{array}{l}\text { A } \\
B\end{array}$ & - & - & - & 0.5 & $\begin{array}{c}5 \\
(2.5) \\
\end{array}$ & 1.20 & 2 & 3.12 & A11 \\
\hline & 11-5a-F8.6-6\#3-i-2.5-3-12 & $\begin{array}{l}A \\
B\end{array}$ & 12 & $\begin{array}{c}4 \\
(2) \\
\end{array}$ & 0.66 & 0.5 & $\begin{array}{c}5 \\
(2.5) \\
\end{array}$ & 1.20 & 2 & 3.12 & A11 \\
\hline \multirow{9}{*}{ Group 16} & 8-8-F4.1-0-i-2.5-3-10-DB & $\begin{array}{l}\text { A } \\
\text { B }\end{array}$ & - & - & - & 0.5 & $\begin{array}{c}5 \\
(2.5)\end{array}$ & 0.80 & 2 & 1.58 & A12 \\
\hline & 8-8-F9.1-0-i-2.5-3-10-DB & $\begin{array}{l}\text { A } \\
\text { B }\end{array}$ & - & - & - & 0.5 & $\begin{array}{c}5 \\
(2.5)\end{array}$ & 0.80 & 2 & 1.58 & A12 \\
\hline & 8-8-F9.1-5\#3-i-2.5-3-10-DB & $\begin{array}{l}\mathrm{A} \\
\mathrm{B}\end{array}$ & 10 & $\begin{array}{c}5 \\
(2.5) \\
\end{array}$ & 0.44 & 0.5 & $\begin{array}{c}5 \\
(2.5) \\
\end{array}$ & 0.80 & 2 & 1.58 & A12 \\
\hline & 8-5-F4.1-0-i-2.5-3-10-DB & $\begin{array}{l}A \\
B\end{array}$ & - & - & - & 0.5 & $\begin{array}{c}5 \\
(2.5)\end{array}$ & 0.80 & 2 & 1.58 & A12 \\
\hline & 8-5-F9.1-0-i-2.5-3-10-DB & $\begin{array}{l}\text { A } \\
\text { B }\end{array}$ & - & - & - & 0.5 & $\begin{array}{c}5 \\
(2.5)\end{array}$ & 0.80 & 2 & 1.58 & A12 \\
\hline & 8-5-F4.1-3\#4-i-2.5-3-10-DB & $\begin{array}{l}\text { A } \\
\text { B }\end{array}$ & 6 & $\begin{array}{l}7.5 \\
(6)\end{array}$ & 0.40 & 0.5 & $\begin{array}{c}5 \\
(2.5)\end{array}$ & 0.80 & 2 & 1.58 & A12 \\
\hline & 8-5-F9.1-3\#4-i-2.5-3-10-DB & $\begin{array}{l}\mathrm{A} \\
\mathrm{B}\end{array}$ & 6 & $\begin{array}{l}7.5 \\
(6)\end{array}$ & 0.40 & 0.5 & $\begin{array}{c}5 \\
(2.5) \\
\end{array}$ & 0.80 & 2 & 1.58 & A12 \\
\hline & 8-5-F4.1-5\#3-i-2.5-3-10-DB & $\begin{array}{l}A \\
B\end{array}$ & 10 & $\begin{array}{c}5 \\
(2.5) \\
\end{array}$ & 0.44 & 0.5 & $\begin{array}{c}5 \\
(2.5) \\
\end{array}$ & 0.80 & 2 & 1.58 & A12 \\
\hline & 8-5-F9.1-5\#3-i-2.5-3-10-DB & $\begin{array}{l}\text { A } \\
B\end{array}$ & 10 & $\begin{array}{c}5 \\
(2.5)\end{array}$ & 0.44 & 0.5 & $\begin{array}{c}5 \\
(2.5)\end{array}$ & 0.80 & 2 & 1.58 & A12 \\
\hline \multirow{6}{*}{ Group 17} & 11-8-F3.8-0-i-2.5-3-14.5 & $\begin{array}{l}\text { A } \\
\text { B }\end{array}$ & - & - & - & 0.5 & $\begin{array}{c}5 \\
(2.5)\end{array}$ & 1.20 & 2 & 3.12 & A12 \\
\hline & 11-8-F3.8-2\#3-i-2.5-3-14.5 & $\begin{array}{l}\text { A } \\
\text { B }\end{array}$ & 4 & $\begin{array}{c}8 \\
(6) \\
\end{array}$ & 0.22 & 0.5 & $\begin{array}{c}5 \\
(2.5) \\
\end{array}$ & 1.20 & 2 & 3.12 & A12 \\
\hline & 11-8-F3.8-6\#3-i-2.5-3-14.5 & $\begin{array}{l}\text { A } \\
B\end{array}$ & 12 & $\begin{array}{c}4 \\
(2) \\
\end{array}$ & 0.66 & 0.5 & $\begin{array}{c}5 \\
(2.5) \\
\end{array}$ & 1.20 & 2 & 3.12 & A12 \\
\hline & (3@5.35)11-8-F3.8-0-i-2.5-3-14.5 & $\begin{array}{l}\text { A } \\
\text { B } \\
\text { C }\end{array}$ & - & - & - & 0.5 & $\begin{array}{c}5 \\
(2.5)\end{array}$ & 1.20 & 3 & 4.68 & A13 \\
\hline & (3@5.35)11-8-F3.8-2\#3-i-2.5-3-14.5 & $\begin{array}{l}\text { A } \\
B \\
C\end{array}$ & 4 & $\begin{array}{c}8 \\
(6)\end{array}$ & 0.22 & 0.5 & $\begin{array}{c}5 \\
(2.5)\end{array}$ & 1.20 & 3 & 4.68 & A14 \\
\hline & (3@5.35)11-8-F3.8-6\#3-i-2.5-3-14.5 & $\begin{array}{l}\text { A } \\
\text { B } \\
\text { C }\end{array}$ & 12 & $\begin{array}{c}4 \\
(2)\end{array}$ & 0.66 & 0.5 & $\begin{array}{c}5 \\
(2.5)\end{array}$ & 1.20 & 3 & 4.68 & A14 \\
\hline
\end{tabular}

${ }^{*}$ Value in parenthesis is the spacing between the first hoop and the center of the headed bar 
Table A.3 Cont. Comprehensive test results and data for beam-column joint specimens

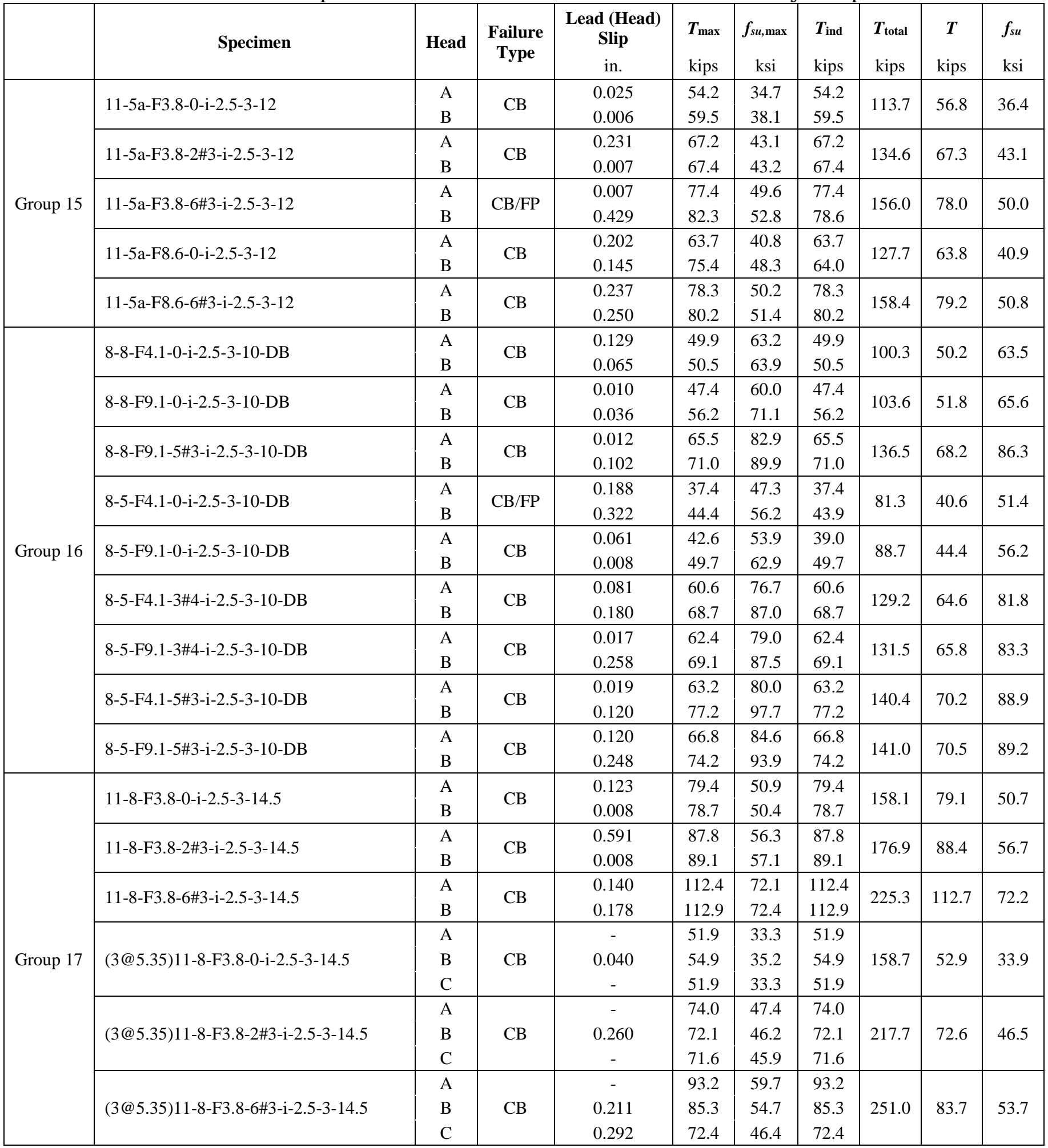


Table A.3 Cont. Comprehensive test results and data for beam-column joint specimens

\begin{tabular}{|c|c|c|c|c|c|c|c|c|c|c|}
\hline & Specimen & Head & $\begin{array}{l}c_{o} \\
\text { in. }\end{array}$ & $A_{b r g}$ & $\begin{array}{l}\ell \text { eh } \\
\text { in. }\end{array}$ & $\begin{array}{c}\ell \text { eh,avg } \\
\text { in. }\end{array}$ & $\begin{array}{l}\boldsymbol{f}_{\boldsymbol{c m}} \\
\mathrm{psi}\end{array}$ & $\begin{array}{l}\text { Age } \\
\text { days }\end{array}$ & $\begin{array}{l}\boldsymbol{d}_{\boldsymbol{b}} \\
\text { in. }\end{array}$ & $\begin{array}{l}\boldsymbol{A}_{\boldsymbol{b}} \\
\text { in. }^{2}\end{array}$ \\
\hline \multirow{4}{*}{ Group 17} & 8-8-O12.9-0-i-2.5-3-9.5 & $\begin{array}{l}\text { A } \\
B\end{array}$ & 1.0 & $12.9 A_{b}$ & $\begin{array}{l}9.75 \\
9.63\end{array}$ & 9.69 & 8800 & 21 & 1 & 0.79 \\
\hline & 8-8-S14.9-0-i-2.5-3-8.25 & $\begin{array}{l}\mathrm{A} \\
\mathrm{B}\end{array}$ & 1.0 & $14.9 A_{b}$ & $\begin{array}{l}8.25 \\
8.25 \\
\end{array}$ & 8.25 & 8800 & 21 & 1 & 0.79 \\
\hline & 8-8-O12.9-5\#3-i-2.5-3-9.5 & $\begin{array}{l}\text { A } \\
B\end{array}$ & 1.0 & $12.9 A_{b}$ & $\begin{array}{l}9.50 \\
9.25\end{array}$ & 9.38 & 8800 & 21 & 1 & 0.79 \\
\hline & 8-8-S14.9-5\#3-i-2.5-3-8.25 & $\begin{array}{l}\text { A } \\
B\end{array}$ & 1.0 & $14.9 A_{b}$ & $\begin{array}{l}8.25 \\
8.25 \\
\end{array}$ & 8.25 & 8800 & 21 & 1 & 0.79 \\
\hline \multirow{8}{*}{ Group 18} & 11-5-F3.8-0-i-2.5-3-12 & $\begin{array}{l}\text { A } \\
B\end{array}$ & 2.0 & $3.8 A_{b}$ & $\begin{array}{l}12.13 \\
12.13 \\
\end{array}$ & 12.13 & 5760 & 6 & 1.41 & 1.56 \\
\hline & 11-5-F3.8-6\#3-i-2.5-3-12 & $\begin{array}{l}\mathrm{A} \\
\mathrm{B}\end{array}$ & 2.0 & $3.8 A_{b}$ & $\begin{array}{l}12.50 \\
12.50 \\
\end{array}$ & 12.50 & 5760 & 6 & 1.41 & 1.56 \\
\hline & 11-5-F3.8-0-i-2.5-3-17 & $\begin{array}{l}\text { A } \\
B\end{array}$ & 2.0 & $3.8 A_{b}$ & $\begin{array}{l}17.50 \\
17.00\end{array}$ & 17.25 & 5760 & 6 & 1.41 & 1.56 \\
\hline & 11-5-F3.8-6\#3-i-2.5-3-17 & $\begin{array}{l}\mathrm{A} \\
\mathrm{B}\end{array}$ & 2.0 & $3.8 A_{b}$ & $\begin{array}{l}16.88 \\
17.00 \\
\end{array}$ & 16.94 & 5970 & 7 & 1.41 & 1.56 \\
\hline & 11-5-F8.6-0-i-2.5-3-14.5 & $\begin{array}{l}\text { A } \\
B\end{array}$ & 2.0 & $8.6 A_{b}$ & $\begin{array}{l}14.50 \\
14.50\end{array}$ & 14.50 & 5970 & 7 & 1.41 & 1.56 \\
\hline & 11-5-F8.6-6\#3-i-2.5-3-14.5 & $\begin{array}{l}\text { A } \\
B\end{array}$ & 2.0 & $8.6 A_{b}$ & $\begin{array}{l}14.50 \\
14.75\end{array}$ & 14.63 & 5970 & 7 & 1.41 & 1.56 \\
\hline & (3@5.35)11-5-F8.6-0-i-2.5-3-14.5 & $\begin{array}{l}\text { A } \\
B \\
C\end{array}$ & 2.0 & $8.6 A_{b}$ & $\begin{array}{l}14.38 \\
15.25 \\
14.50\end{array}$ & 14.71 & 6240 & 8 & 1.41 & 1.56 \\
\hline & (3@5.35)11-5-F8.6-6\#3-i-2.5-3-14.5 & $\begin{array}{l}\text { A } \\
B \\
C\end{array}$ & 2.0 & $8.6 A_{b}$ & $\begin{array}{l}14.75 \\
14.50 \\
14.38\end{array}$ & 14.54 & 6240 & 8 & 1.41 & 1.56 \\
\hline \multirow{8}{*}{ Group 19} & $11-12-04.5-0-\mathrm{i}-2.5-3-16.75$ & $\begin{array}{l}\text { A } \\
B\end{array}$ & 1.3 & $4.5 A_{b}$ & $\begin{array}{l}17.13 \\
17.13\end{array}$ & 17.13 & 10860 & 36 & 1.41 & 1.56 \\
\hline & 11-12-S5.5-0-i-2.5-3-16.75 & $\begin{array}{l}\text { A } \\
B\end{array}$ & 1.5 & $5.5 A_{b}$ & $\begin{array}{l}16.75 \\
17.13\end{array}$ & 16.94 & 10120 & 37 & 1.41 & 1.56 \\
\hline & 11-12-O4.5-6\#3-i-2.5-3-16.75 & $\begin{array}{l}\mathrm{A} \\
\mathrm{B}\end{array}$ & 1.3 & $4.5 A_{b}$ & $\begin{array}{l}16.75 \\
16.88 \\
\end{array}$ & 16.81 & 10860 & 37 & 1.41 & 1.56 \\
\hline & 11-12-S5.5-6\#3-i-2.5-3-16.75 & $\begin{array}{l}\text { A } \\
B\end{array}$ & 1.5 & $5.5 A_{b}$ & $\begin{array}{l}16.63 \\
17.00\end{array}$ & 16.81 & 10120 & 38 & 1.41 & 1.56 \\
\hline & (3@5.35)11-12-04.5-0-i-2.5-3-16.75 & $\begin{array}{l}\text { A } \\
B \\
C\end{array}$ & 1.3 & $4.5 A_{b}$ & $\begin{array}{l}16.88 \\
17.13 \\
16.75\end{array}$ & 16.92 & 10860 & 36 & 1.41 & 1.56 \\
\hline & (3@5.35)11-12-S5.5-0-i-2.5-3-16.75 & $\begin{array}{l}\text { A } \\
B \\
C\end{array}$ & 1.5 & $5.5 A_{b}$ & $\begin{array}{l}16.88 \\
17.00 \\
16.88\end{array}$ & 16.92 & 10120 & 38 & 1.41 & 1.56 \\
\hline & (3@5.35)11-12-O4.5-6\#3-i-2.5-3-16.75 & $\begin{array}{l}\text { A } \\
\text { B } \\
\text { C }\end{array}$ & 1.3 & $4.5 A_{b}$ & $\begin{array}{l}16.88 \\
17.13 \\
17.00 \\
\end{array}$ & 17.00 & 10860 & 37 & 1.41 & 1.56 \\
\hline & (3@5.35)11-12-S5.5-6\#3-i-2.5-3-16.75 & $\begin{array}{l}\text { A } \\
B \\
C\end{array}$ & 1.5 & $5.5 A_{b}$ & $\begin{array}{l}16.75 \\
17.00 \\
16.50\end{array}$ & 16.75 & 10120 & 38 & 1.41 & 1.56 \\
\hline
\end{tabular}


Table A.3 Cont. Comprehensive test results and data for beam-column joint specimens

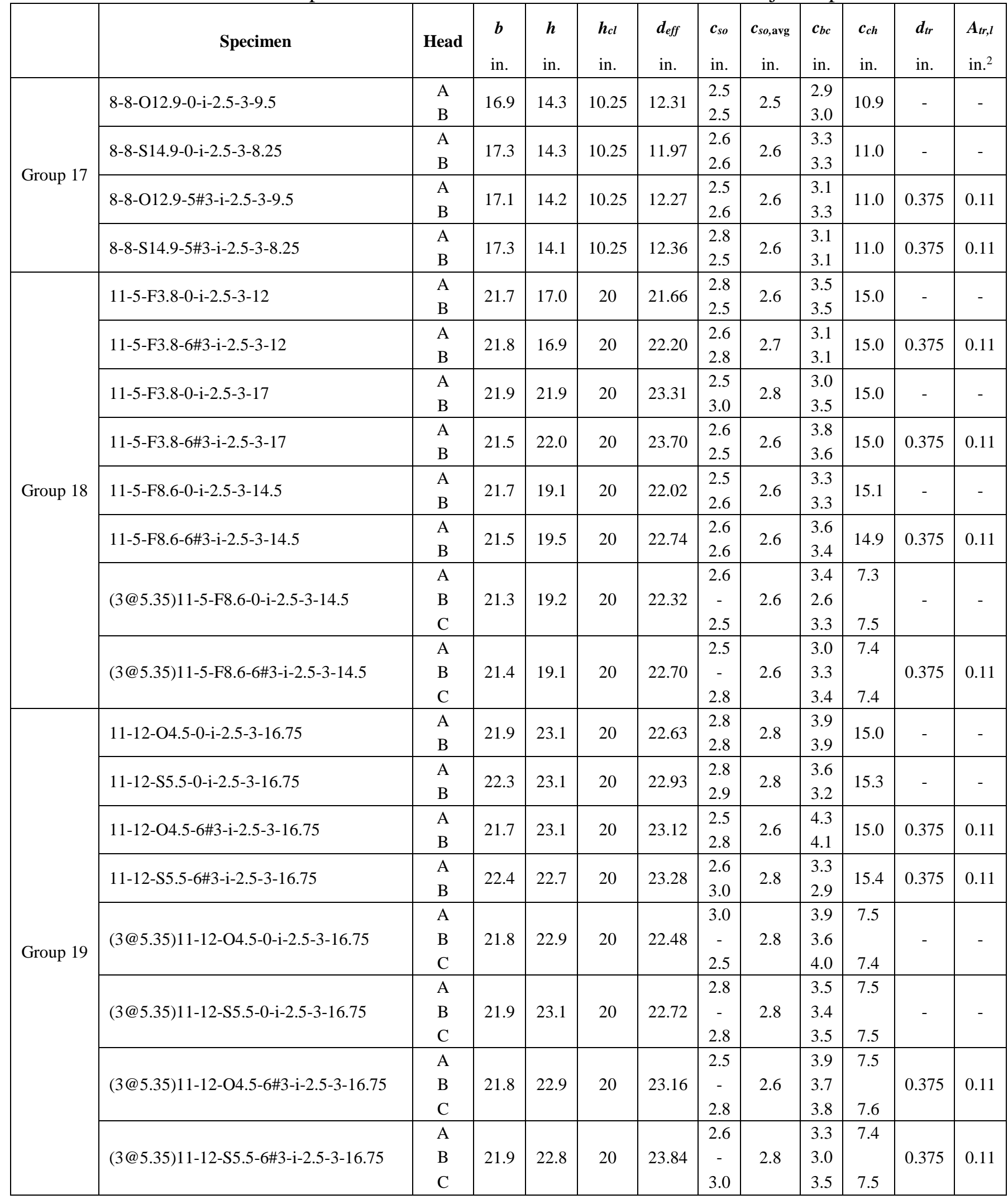


Table A.3 Cont. Comprehensive test results and data for beam-column joint specimens

\begin{tabular}{|c|c|c|c|c|c|c|c|c|c|c|c|}
\hline & Specimen & Head & $N$ & $\begin{array}{l}\boldsymbol{s}_{t r}^{*} \\
\text { in. }\end{array}$ & $\begin{array}{l}\boldsymbol{A}_{t t} \\
\text { in. }^{2}\end{array}$ & $\begin{array}{l}\boldsymbol{d}_{\text {tro }} \\
\text { in. }\end{array}$ & $\begin{array}{l}\text { Stro }^{*} \\
\text { in. }\end{array}$ & $\begin{array}{l}A_{a b} \\
\text { in. }^{2}\end{array}$ & $n$ & $\begin{array}{l}\text { A }_{h s} \\
\text { in. }^{2}\end{array}$ & $\begin{array}{c}\text { Long. } \\
\text { Reinf. } \\
\text { Layout }\end{array}$ \\
\hline \multirow{4}{*}{ Group 17} & 8-8-O12.9-0-i-2.5-3-9.5 & $\begin{array}{l}\text { A } \\
\text { B }\end{array}$ & - & - & - & 0.5 & $\begin{array}{c}4.5 \\
(2.25)\end{array}$ & 0.80 & 2 & 1.58 & A6 \\
\hline & 8-8-S14.9-0-i-2.5-3-8.25 & $\begin{array}{l}\text { A } \\
B\end{array}$ & - & - & - & 0.5 & $\begin{array}{c}4.5 \\
(2.25)\end{array}$ & 0.80 & 2 & 1.58 & A6 \\
\hline & 8-8-012.9-5\#3-i-2.5-3-9.5 & $\begin{array}{l}\text { A } \\
B\end{array}$ & 10 & $\begin{array}{c}3 \\
(1.5) \\
\end{array}$ & 0.66 & 0.5 & $\begin{array}{c}4.5 \\
(2.25)\end{array}$ & 0.80 & 2 & 1.58 & A6 \\
\hline & 8-8-S14.9-5\#3-i-2.5-3-8.25 & $\begin{array}{l}\text { A } \\
B\end{array}$ & 10 & $\begin{array}{c}3 \\
(1.5) \\
\end{array}$ & 0.66 & 0.5 & $\begin{array}{c}4.5 \\
(2.25)\end{array}$ & 0.80 & 2 & 1.58 & A6 \\
\hline \multirow{8}{*}{ Group 18} & 11-5-F3.8-0-i-2.5-3-12 & $\begin{array}{l}\mathrm{A} \\
\mathrm{B}\end{array}$ & - & - & - & 0.5 & $\begin{array}{c}5 \\
(2.5) \\
\end{array}$ & 1.20 & 2 & 3.12 & A6 \\
\hline & 11-5-F3.8-6\#3-i-2.5-3-12 & $\begin{array}{l}A \\
B\end{array}$ & 12 & $\begin{array}{c}4 \\
(2) \\
\end{array}$ & 0.66 & 0.5 & $\begin{array}{c}5 \\
(2.5)\end{array}$ & 1.20 & 2 & 3.12 & A12 \\
\hline & 11-5-F3.8-0-i-2.5-3-17 & $\begin{array}{l}A \\
B\end{array}$ & - & - & - & 0.5 & $\begin{array}{c}6 \\
(3) \\
\end{array}$ & 0.80 & 2 & 3.12 & A7 \\
\hline & 11-5-F3.8-6\#3-i-2.5-3-17 & $\begin{array}{l}\mathrm{A} \\
\mathrm{B}\end{array}$ & 12 & $\begin{array}{c}4 \\
(2) \\
\end{array}$ & 0.66 & 0.5 & $\begin{array}{c}6 \\
(3) \\
\end{array}$ & 0.80 & 2 & 3.12 & A7 \\
\hline & 11-5-F8.6-0-i-2.5-3-14.5 & $\begin{array}{l}\text { A } \\
B\end{array}$ & - & - & - & 0.5 & $\begin{array}{c}5 \\
(2.5)\end{array}$ & 1.20 & 2 & 3.12 & A12 \\
\hline & 11-5-F8.6-6\#3-i-2.5-3-14.5 & $\begin{array}{l}\text { A } \\
B\end{array}$ & 12 & $\begin{array}{c}4 \\
(2)\end{array}$ & 0.66 & 0.5 & $\begin{array}{c}5 \\
(2.5)\end{array}$ & 1.20 & 2 & 3.12 & A12 \\
\hline & (3@5.35)11-5-F8.6-0-i-2.5-3-14.5 & $\begin{array}{l}\text { A } \\
\text { B } \\
\text { C }\end{array}$ & - & - & - & 0.5 & $\begin{array}{c}5 \\
(2.5)\end{array}$ & 1.20 & 3 & 4.68 & A15 \\
\hline & (3@5.35)11-5-F8.6-6\#3-i-2.5-3-14.5 & $\begin{array}{l}\text { A } \\
\text { B } \\
\text { C }\end{array}$ & 12 & $\begin{array}{c}4 \\
(2)\end{array}$ & 0.66 & 0.5 & $\begin{array}{c}4.5 \\
(2.25)\end{array}$ & 1.20 & 3 & 4.68 & A15 \\
\hline \multirow{8}{*}{ Group 19} & $11-12-04.5-0-\mathrm{i}-2.5-3-16.75$ & $\begin{array}{l}\text { A } \\
B\end{array}$ & - & - & - & 0.5 & $\begin{array}{c}4 \\
(2)\end{array}$ & 1.20 & 2 & 3.12 & A16 \\
\hline & $11-12-S 5.5-0-\mathrm{i}-2.5-3-16.75$ & $\begin{array}{l}\text { A } \\
\text { B }\end{array}$ & - & - & - & 0.5 & $\begin{array}{c}4 \\
(2) \\
\end{array}$ & 1.20 & 2 & 3.12 & A16 \\
\hline & 11-12-04.5-6\#3-i-2.5-3-16.75 & $\begin{array}{l}\text { A } \\
\text { B }\end{array}$ & 12 & $\begin{array}{c}4 \\
(2) \\
\end{array}$ & 0.66 & 0.5 & $\begin{array}{c}4 \\
(2) \\
\end{array}$ & 1.20 & 2 & 3.12 & A16 \\
\hline & 11-12-S5.5-6\#3-i-2.5-3-16.75 & $\begin{array}{l}\mathrm{A} \\
\mathrm{B}\end{array}$ & 12 & $\begin{array}{c}4 \\
(2) \\
\end{array}$ & 0.66 & 0.5 & $\begin{array}{c}4 \\
(2) \\
\end{array}$ & 1.20 & 2 & 3.12 & A16 \\
\hline & (3@5.35)11-12-04.5-0-i-2.5-3-16.75 & $\begin{array}{l}\text { A } \\
\text { B } \\
\text { C }\end{array}$ & - & - & - & 0.5 & $\begin{array}{c}4 \\
(2)\end{array}$ & 1.20 & 3 & 4.68 & A16 \\
\hline & (3@5.35)11-12-S5.5-0-i-2.5-3-16.75 & $\begin{array}{l}\text { A } \\
\text { B } \\
\text { C }\end{array}$ & - & - & - & 0.5 & $\begin{array}{c}4 \\
(2)\end{array}$ & 1.20 & 3 & 4.68 & A16 \\
\hline & (3@5.35)11-12-O4.5-6\#3-i-2.5-3-16.75 & $\begin{array}{l}\text { A } \\
\text { B } \\
\text { C }\end{array}$ & 12 & $\begin{array}{c}4 \\
(2)\end{array}$ & 0.66 & 0.5 & $\begin{array}{c}4 \\
(2)\end{array}$ & 1.20 & 3 & 4.68 & A16 \\
\hline & (3@5.35)11-12-S5.5-6\#3-i-2.5-3-16.75 & $\begin{array}{l}A \\
B \\
C\end{array}$ & 12 & $\begin{array}{c}4 \\
(2)\end{array}$ & 0.66 & 0.5 & $\begin{array}{c}4 \\
(2)\end{array}$ & 1.20 & 3 & 4.68 & A16 \\
\hline
\end{tabular}

${ }^{*}$ Value in parenthesis is the spacing between the first hoop and the center of the headed bar 
Table A.3 Cont. Comprehensive test results and data for beam-column joint specimens

\begin{tabular}{|c|c|c|c|c|c|c|c|c|c|c|}
\hline & Specimen & Head & $\begin{array}{c}\text { Failure } \\
\text { Type }\end{array}$ & $\begin{array}{c}\text { Lead (Head) } \\
\text { Slip } \\
\text { in. }\end{array}$ & $\begin{array}{l}\boldsymbol{T}_{\max } \\
\text { kips }\end{array}$ & $\begin{array}{c}\boldsymbol{f}_{\text {su,max }} \\
\text { ksi }\end{array}$ & $\begin{array}{l}\boldsymbol{T}_{\text {ind }} \\
\text { kips }\end{array}$ & $\begin{array}{l}\boldsymbol{T}_{\text {total }} \\
\text { kips }\end{array}$ & $\begin{array}{c}\boldsymbol{T} \\
\text { kips }\end{array}$ & $\begin{array}{l}f_{s u} \\
\mathrm{ksi}\end{array}$ \\
\hline \multirow{4}{*}{ Group 17} & 8-8-012.9-0-i-2.5-3-9.5 & $\begin{array}{l}\text { A } \\
\text { B }\end{array}$ & CB & - & $\begin{array}{l}85.5 \\
84.9\end{array}$ & $\begin{array}{l}108.2 \\
107.5\end{array}$ & $\begin{array}{l}85.5 \\
84.9\end{array}$ & 170.5 & 85.2 & 107.8 \\
\hline & $8-8-S 14.9-0-\mathrm{i}-2.5-3-8.25$ & $\begin{array}{l}\mathrm{A} \\
\mathrm{B}\end{array}$ & $\mathrm{CB}$ & $\begin{array}{l}0.010 \\
0.187\end{array}$ & $\begin{array}{l}70.8 \\
71.0\end{array}$ & $\begin{array}{l}89.6 \\
89.9\end{array}$ & $\begin{array}{l}70.8 \\
71.0\end{array}$ & 141.8 & 70.9 & 89.7 \\
\hline & 8-8-O12.9-5\#3-i-2.5-3-9.5 & $\begin{array}{l}\mathrm{A} \\
\mathrm{B}\end{array}$ & CB & $\begin{array}{l}0.237 \\
0.198\end{array}$ & $\begin{array}{l}84.4 \\
82.5\end{array}$ & $\begin{array}{l}106.8 \\
104.4\end{array}$ & $\begin{array}{l}84.4 \\
82.5\end{array}$ & 166.9 & 83.5 & 105.7 \\
\hline & 8-8-S14.9-5\#3-i-2.5-3-8.25 & $\begin{array}{l}\text { A } \\
\text { B }\end{array}$ & CB & $\begin{array}{l}0.197 \\
0.022\end{array}$ & $\begin{array}{l}87.2 \\
86.8\end{array}$ & $\begin{array}{l}110.4 \\
109.9\end{array}$ & $\begin{array}{l}87.2 \\
86.8\end{array}$ & 174.0 & 87.0 & 110.1 \\
\hline \multirow{8}{*}{ Group 18} & 11-5-F3.8-0-i-2.5-3-12 & $\begin{array}{l}\text { A } \\
\text { B }\end{array}$ & CB & $\begin{array}{l}0.140 \\
0.262\end{array}$ & $\begin{array}{l}68.7 \\
64.3\end{array}$ & $\begin{array}{l}44.0 \\
41.2\end{array}$ & $\begin{array}{l}68.7 \\
64.3\end{array}$ & 132.9 & 66.5 & 42.6 \\
\hline & 11-5-F3.8-6\#3-i-2.5-3-12 & $\begin{array}{l}\mathrm{A} \\
\mathrm{B}\end{array}$ & CB & $\begin{array}{l}0.041 \\
0.008 \\
\end{array}$ & $\begin{array}{l}88.2 \\
88.3 \\
\end{array}$ & $\begin{array}{l}56.5 \\
56.6 \\
\end{array}$ & $\begin{array}{l}88.2 \\
88.3 \\
\end{array}$ & 176.5 & 88.3 & 56.6 \\
\hline & 11-5-F3.8-0-i-2.5-3-17 & $\begin{array}{l}\text { A } \\
\text { B }\end{array}$ & $\mathrm{CB} / \mathrm{FP}$ & $\begin{array}{l}0.115 \\
0.015\end{array}$ & $\begin{array}{l}132.6 \\
132.9\end{array}$ & $\begin{array}{l}85.0 \\
85.2\end{array}$ & $\begin{array}{l}132.6 \\
132.9\end{array}$ & 265.5 & 132.7 & 85.1 \\
\hline & 11-5-F3.8-6\#3-i-2.5-3-17 & $\begin{array}{l}\text { A } \\
\text { B }\end{array}$ & CB & $\begin{array}{l}0.157 \\
0.051\end{array}$ & $\begin{array}{l}154.9 \\
148.9\end{array}$ & $\begin{array}{l}99.3 \\
95.4\end{array}$ & $\begin{array}{l}154.9 \\
148.9\end{array}$ & 303.7 & 151.9 & 97.4 \\
\hline & 11-5-F8.6-0-i-2.5-3-14.5 & $\begin{array}{l}\text { A } \\
\text { B }\end{array}$ & CB & $\begin{array}{l}0.005 \\
0.783\end{array}$ & $\begin{array}{l}83.6 \\
82.1\end{array}$ & $\begin{array}{l}53.6 \\
52.6\end{array}$ & $\begin{array}{l}83.6 \\
82.1\end{array}$ & 165.7 & 82.8 & 53.1 \\
\hline & 11-5-F8.6-6\#3-i-2.5-3-14.5 & $\begin{array}{l}\text { A } \\
\text { B }\end{array}$ & $\mathrm{CB}$ & $\begin{array}{l}0.144 \\
0.010\end{array}$ & $\begin{array}{l}113.9 \\
110.7\end{array}$ & $\begin{array}{l}73.0 \\
71.0\end{array}$ & $\begin{array}{l}113.9 \\
110.7\end{array}$ & 224.6 & 112.3 & 72.0 \\
\hline & (3@5.35)11-5-F8.6-0-i-2.5-3-14.5 & $\begin{array}{l}\text { A } \\
\text { B } \\
\text { C }\end{array}$ & CB & $\begin{array}{c}- \\
0.013 \\
0.068\end{array}$ & $\begin{array}{l}66.5 \\
61.7 \\
67.1\end{array}$ & $\begin{array}{l}42.6 \\
39.6 \\
43.0\end{array}$ & $\begin{array}{l}66.5 \\
61.7 \\
67.1\end{array}$ & 195.4 & 65.1 & 41.7 \\
\hline & (3@5.35)11-5-F8.6-6\#3-i-2.5-3-14.5 & $\begin{array}{l}\text { A } \\
\text { B } \\
\text { C }\end{array}$ & CB & $\begin{array}{c}- \\
0.287 \\
0.016\end{array}$ & $\begin{array}{l}68.8 \\
83.3 \\
74.9\end{array}$ & $\begin{array}{l}44.1 \\
53.4 \\
48.0\end{array}$ & $\begin{array}{l}68.8 \\
83.2 \\
74.9\end{array}$ & 226.9 & 75.6 & 48.5 \\
\hline \multirow{8}{*}{ Group 19} & $11-12-04.5-0-\mathrm{i}-2.5-3-16.75$ & $\begin{array}{l}\text { A } \\
\text { B }\end{array}$ & CB & $\begin{array}{l}0.032 \\
0.029\end{array}$ & $\begin{array}{l}168.4 \\
171.0\end{array}$ & $\begin{array}{l}107.9 \\
109.6\end{array}$ & $\begin{array}{l}168.3 \\
171.0\end{array}$ & 339.3 & 169.6 & 108.7 \\
\hline & $11-12-S 5.5-0-\mathrm{i}-2.5-3-16.75$ & $\begin{array}{l}\text { A } \\
\text { B }\end{array}$ & CB & $\begin{array}{l}0.091 \\
0.215\end{array}$ & $\begin{array}{l}179.1 \\
172.7\end{array}$ & $\begin{array}{l}114.8 \\
110.7\end{array}$ & $\begin{array}{l}179.1 \\
172.7\end{array}$ & 351.9 & 175.9 & 112.8 \\
\hline & 11-12-O4.5-6\#3-i-2.5-3-16.75 & $\begin{array}{l}\text { A } \\
\text { B }\end{array}$ & $\mathrm{SB} / \mathrm{FP}$ & $\begin{array}{l}0.024 \\
0.022\end{array}$ & $\begin{array}{l}202.5 \\
200.7^{\ddagger}\end{array}$ & $\begin{array}{l}129.8 \\
128.7^{\ddagger}\end{array}$ & $\begin{array}{l}202.5 \\
200.5\end{array}$ & 403.0 & 201.5 & 129.2 \\
\hline & 11-12-S5.5-6\#3-i-2.5-3-16.75 & $\begin{array}{l}\mathrm{A} \\
\mathrm{B}\end{array}$ & $\mathrm{CB}$ & $\begin{array}{l}0.028 \\
0.025 \\
\end{array}$ & $\begin{array}{l}206.1 \\
188.7 \\
\end{array}$ & $\begin{array}{l}132.1 \\
121.0 \\
\end{array}$ & $\begin{array}{l}206.1 \\
188.7\end{array}$ & 394.8 & 197.4 & 126.5 \\
\hline & (3@5.35)11-12-O4.5-0-i-2.5-3-16.75 & $\begin{array}{l}\mathrm{A} \\
\mathrm{B} \\
\mathrm{C}\end{array}$ & CB & $\begin{array}{c}- \\
0.003 \\
0.003 \\
\end{array}$ & $\begin{array}{c}109.3 \\
114.1 \\
98.7 \\
\end{array}$ & $\begin{array}{l}70.1 \\
73.1 \\
63.3 \\
\end{array}$ & $\begin{array}{c}107.6 \\
114.1 \\
98.7 \\
\end{array}$ & 320.4 & 106.8 & 68.5 \\
\hline & (3@5.35)11-12-S5.5-0-i-2.5-3-16.75 & $\begin{array}{l}\text { A } \\
\text { B } \\
\text { C }\end{array}$ & CB & $\begin{array}{l}- \\
- \\
-\end{array}$ & $\begin{array}{c}117.1 \\
93.8 \\
116.1\end{array}$ & $\begin{array}{l}75.1 \\
60.1 \\
74.4\end{array}$ & $\begin{array}{c}117.1 \\
93.8 \\
116.1\end{array}$ & 327.0 & 109.0 & 69.9 \\
\hline & (3@5.35)11-12-04.5-6\#3-i-2.5-3-16.75 & $\begin{array}{l}\text { A } \\
\text { B } \\
\text { C }\end{array}$ & CB & $\begin{array}{c}- \\
0.213 \\
0.145 \\
\end{array}$ & $\begin{array}{l}131.7 \\
131.8 \\
143.9 \\
\end{array}$ & $\begin{array}{l}84.4 \\
84.5 \\
92.2 \\
\end{array}$ & $\begin{array}{l}131.7 \\
131.8 \\
143.9 \\
\end{array}$ & 407.4 & 135.8 & 87.1 \\
\hline & (3@5.35)11-12-S5.5-6\#3-i-2.5-3-16.75 & $\begin{array}{l}\text { A } \\
\text { B } \\
\text { C }\end{array}$ & CB & $\begin{array}{c}- \\
0.095 \\
-\end{array}$ & $\begin{array}{l}155.9 \\
154.9 \\
150.6\end{array}$ & $\begin{array}{l}99.9 \\
99.3 \\
96.5\end{array}$ & $\begin{array}{l}155.9 \\
154.9 \\
150.6\end{array}$ & 461.3 & 153.8 & 98.6 \\
\hline
\end{tabular}

${ }^{\ddagger}$ No anchorage failure on the bar 
Table A.3 Cont. Comprehensive test results and data for beam-column joint specimens

\begin{tabular}{|c|c|c|c|c|c|c|c|c|c|c|}
\hline & Specimen & Head & $\begin{array}{l}c_{o} \\
\text { in. }\end{array}$ & $A_{b r g}$ & $\begin{array}{l}\ell \text { eh } \\
\text { in. }\end{array}$ & $\begin{array}{c}\ell e h, \text { avg } \\
\text { in. }\end{array}$ & $\begin{array}{l}f_{c m} \\
\text { psi }\end{array}$ & $\begin{array}{l}\text { Age } \\
\text { days }\end{array}$ & $\begin{array}{l}d_{b} \\
\text { in. }\end{array}$ & $\begin{array}{l}A_{b} \\
\text { in. }^{2}\end{array}$ \\
\hline \multirow{8}{*}{ Group 20} & $11-5-04.5-0-\mathrm{i}-2.5-3-19.25$ & $\begin{array}{l}\text { A } \\
B\end{array}$ & 1.3 & $4.5 A_{b}$ & $\begin{array}{l}19.63 \\
19.25\end{array}$ & 19.44 & 5430 & 12 & 1.41 & 1.56 \\
\hline & 11-5-S5.5-0-i-2.5-3-19.25 & $\begin{array}{l}\text { A } \\
B\end{array}$ & 1.5 & $5.5 A_{b}$ & $\begin{array}{l}19.38 \\
19.38\end{array}$ & 19.38 & 6320 & 11 & 1.41 & 1.56 \\
\hline & 11-5-O4.5-6\#3-i-2.5-3-19.25 & $\begin{array}{l}\text { A } \\
B\end{array}$ & 1.3 & $4.5 A_{b}$ & $\begin{array}{l}19.50 \\
19.75\end{array}$ & 19.63 & 5430 & 12 & 1.41 & 1.56 \\
\hline & 11-5-S5.5-6\#3-i-2.5-3-19.25 & $\begin{array}{l}\text { A } \\
B\end{array}$ & 1.5 & $5.5 A_{b}$ & $\begin{array}{l}19.13 \\
19.13\end{array}$ & 19.13 & 6320 & 13 & 1.41 & 1.56 \\
\hline & (3@5.35)11-5-O4.5-0-i-2.5-3-19.25 & $\begin{array}{l}A \\
B \\
C\end{array}$ & 1.3 & $4.5 A_{b}$ & $\begin{array}{l}19.50 \\
19.63 \\
19.38\end{array}$ & 19.50 & 5430 & 12 & 1.41 & 1.56 \\
\hline & (3@5.35)11-5-S5.5-0-i-2.5-3-19.25 & $\begin{array}{l}\text { A } \\
B \\
C\end{array}$ & 1.5 & $5.5 A_{b}$ & $\begin{array}{l}19.25 \\
19.38 \\
19.25\end{array}$ & 19.29 & 6320 & 11 & 1.41 & 1.56 \\
\hline & (3@5.35)11-5-O4.5-6\#3-i-2.5-3-19.25 & $\begin{array}{l}\text { A } \\
B \\
C\end{array}$ & 1.3 & $4.5 A_{b}$ & $\begin{array}{l}19.38 \\
19.63 \\
19.13\end{array}$ & 19.38 & 5430 & 13 & 1.41 & 1.56 \\
\hline & (3@5.35)11-5-S5.5-6\#3-i-2.5-3-19.25 & $\begin{array}{l}\text { A } \\
B \\
C\end{array}$ & 1.5 & $5.5 A_{b}$ & $\begin{array}{l}19.00 \\
19.38 \\
19.38\end{array}$ & 19.25 & 6320 & 13 & 1.41 & 1.56 \\
\hline
\end{tabular}

Table A.3 Cont. Comprehensive test results and data for beam-column joint specimens

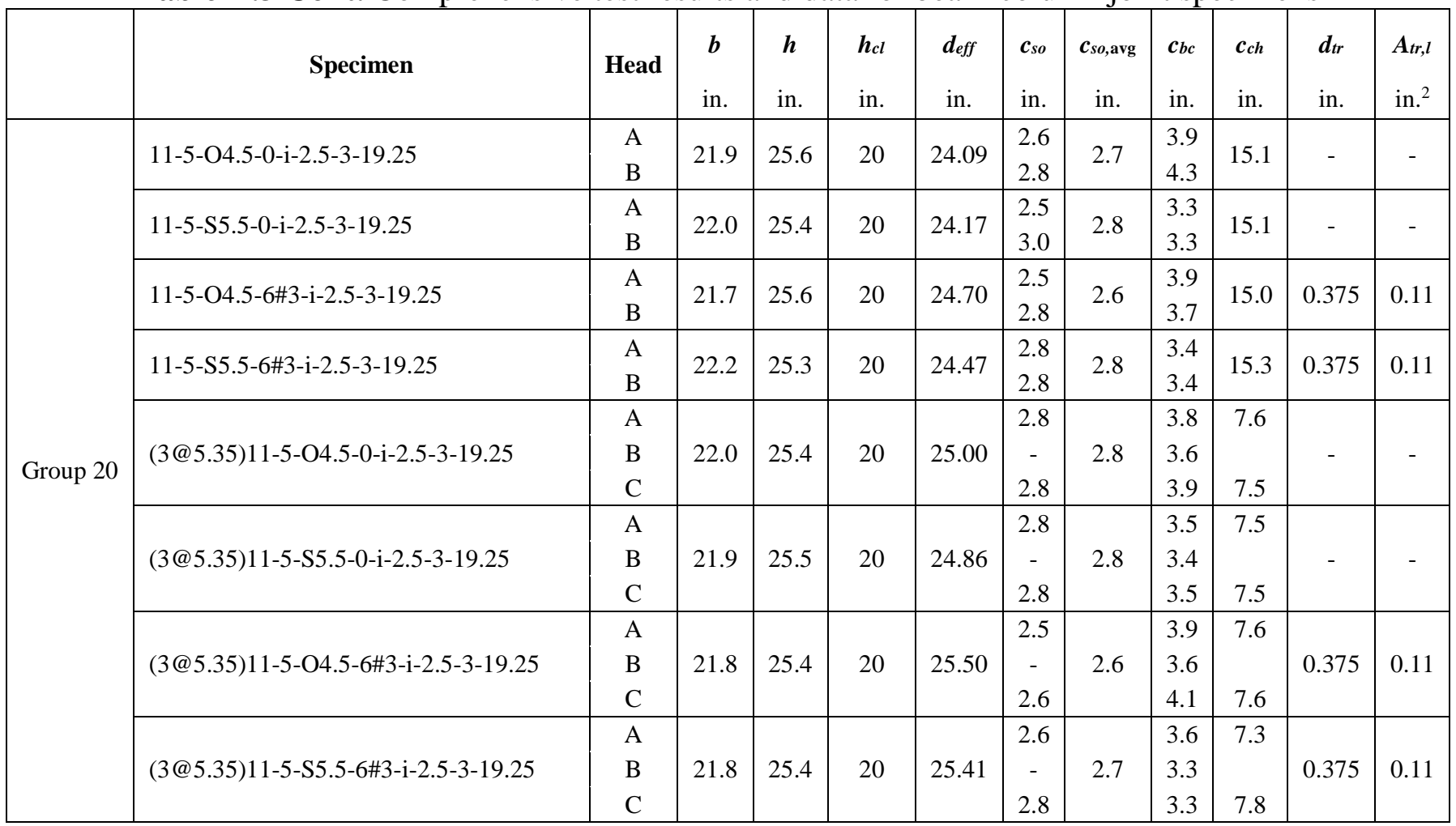


Table A.3 Cont. Comprehensive test results and data for beam-column joint specimens

\begin{tabular}{|c|c|c|c|c|c|c|c|c|c|c|c|}
\hline & Specimen & Head & $N$ & $\begin{array}{l}S_{t r}^{*} \\
\text { in. }\end{array}$ & $\begin{array}{l}\boldsymbol{A}_{t t} \\
\text { in. }^{2}\end{array}$ & $\begin{array}{l}\boldsymbol{d}_{\text {tro }} \\
\text { in. }\end{array}$ & $\begin{array}{c}\text { Stro }^{*} \\
\text { in. }\end{array}$ & $\begin{array}{l}A_{a b} \\
\text { in. }^{2}\end{array}$ & $n$ & $\begin{array}{l}\boldsymbol{A}_{\boldsymbol{h s}} \\
\text { in. }^{2}\end{array}$ & $\begin{array}{l}\text { Long. } \\
\text { Reinf. } \\
\text { Layout }\end{array}$ \\
\hline \multirow{8}{*}{ Group 20} & 11-5-O4.5-0-i-2.5-3-19.25 & $\begin{array}{l}\text { A } \\
B\end{array}$ & - & - & - & 0.5 & $\begin{array}{c}4 \\
(2)\end{array}$ & 1.20 & 2 & 3.12 & A16 \\
\hline & 11-5-S5.5-0-i-2.5-3-19.25 & $\begin{array}{l}\text { A } \\
B\end{array}$ & - & - & - & 0.5 & $\begin{array}{c}4 \\
(2)\end{array}$ & 1.20 & 2 & 3.12 & A16 \\
\hline & 11-5-O4.5-6\#3-i-2.5-3-19.25 & $\begin{array}{l}\text { A } \\
\text { B }\end{array}$ & 12 & $\begin{array}{c}4 \\
(2)\end{array}$ & 0.66 & 0.5 & $\begin{array}{c}4 \\
(2)\end{array}$ & 1.20 & 2 & 3.12 & A16 \\
\hline & 11-5-S5.5-6\#3-i-2.5-3-19.25 & $\begin{array}{l}\text { A } \\
B\end{array}$ & 12 & $\begin{array}{c}4 \\
(2)\end{array}$ & 0.66 & 0.5 & $\begin{array}{c}4 \\
(2)\end{array}$ & 1.20 & 2 & 3.12 & A16 \\
\hline & (3@5.35)11-5-O4.5-0-i-2.5-3-19.25 & $\begin{array}{l}\text { A } \\
\text { B } \\
\text { C }\end{array}$ & - & - & - & 0.5 & $\begin{array}{c}4 \\
(2)\end{array}$ & 1.20 & 3 & 4.68 & A16 \\
\hline & (3@5.35)11-5-S5.5-0-i-2.5-3-19.25 & $\begin{array}{l}\text { A } \\
B \\
C\end{array}$ & - & - & - & 0.5 & $\begin{array}{c}4 \\
(2)\end{array}$ & 1.20 & 3 & 4.68 & A16 \\
\hline & (3@5.35)11-5-O4.5-6\#3-i-2.5-3-19.25 & $\begin{array}{l}\text { A } \\
B \\
C\end{array}$ & 12 & $\begin{array}{c}4 \\
(2)\end{array}$ & 0.66 & 0.5 & $\begin{array}{c}4 \\
(2)\end{array}$ & 1.20 & 3 & 4.68 & A16 \\
\hline & (3@5.35)11-5-S5.5-6\#3-i-2.5-3-19.25 & $\begin{array}{l}A \\
B \\
C\end{array}$ & 12 & $\begin{array}{c}4 \\
(2)\end{array}$ & 0.66 & 0.5 & $\begin{array}{c}4 \\
(2)\end{array}$ & 1.20 & 3 & 4.68 & A16 \\
\hline
\end{tabular}

${ }^{*}$ Value in parenthesis is the spacing between the first hoop and the center of the headed bar

Table A.3 Cont. Comprehensive test results and data for beam-column joint specimens

\begin{tabular}{|c|c|c|c|c|c|c|c|c|c|c|}
\hline & Specimen & Head & $\begin{array}{c}\text { Failure } \\
\text { Type }\end{array}$ & $\begin{array}{c}\text { Lead (Head) } \\
\text { Slip } \\
\text { in. }\end{array}$ & $\begin{array}{l}\boldsymbol{T}_{\max } \\
\text { kips }\end{array}$ & $\begin{array}{c}\boldsymbol{f}_{\text {su,max }} \\
\text { ksi }\end{array}$ & $\begin{array}{l}\boldsymbol{T}_{\text {ind }} \\
\text { kips }\end{array}$ & $\begin{array}{l}\boldsymbol{T}_{\text {total }} \\
\text { kips }\end{array}$ & $\begin{array}{c}\boldsymbol{T} \\
\text { kips }\end{array}$ & $\begin{array}{l}\boldsymbol{f}_{\text {su }} \\
\mathrm{ksi}\end{array}$ \\
\hline \multirow{8}{*}{ Group 20} & $11-5-04.5-0-\mathrm{i}-2.5-3-19.25$ & $\begin{array}{l}\text { A } \\
B\end{array}$ & $\mathrm{SB} / \mathrm{FP}$ & $\begin{array}{l}0.021 \\
0.128\end{array}$ & $\begin{array}{l}161.4 \\
154.4\end{array}$ & $\begin{array}{c}103.5 \\
99.0\end{array}$ & $\begin{array}{l}161.4 \\
154.3\end{array}$ & 315.7 & 157.9 & 101.2 \\
\hline & 11-5-S5.5-0-i-2.5-3-19.25 & $\begin{array}{l}\text { A } \\
B\end{array}$ & $\mathrm{SB} / \mathrm{FP}$ & $\begin{array}{l}0.117 \\
0.095\end{array}$ & $\begin{array}{l}176.9 \\
176.8^{\ddagger}\end{array}$ & $\begin{array}{l}113.4 \\
113.3^{\ddagger}\end{array}$ & $\begin{array}{l}176.9 \\
176.7\end{array}$ & 353.6 & 176.8 & 113.3 \\
\hline & 11-5-O4.5-6\#3-i-2.5-3-19.25 & $\begin{array}{l}\text { A } \\
B\end{array}$ & $\mathrm{SB} / \mathrm{FP}$ & $\begin{array}{l}0.012 \\
0.036\end{array}$ & $\begin{array}{l}180.4^{\ddagger} \\
182.6\end{array}$ & $\begin{array}{l}115.6^{\ddagger} \\
117.1\end{array}$ & $\begin{array}{l}180.3 \\
182.6\end{array}$ & 362.9 & 181.4 & 116.3 \\
\hline & 11-5-S5.5-6\#3-i-2.5-3-19.25 & $\begin{array}{l}\text { A } \\
\text { B }\end{array}$ & $\mathrm{SB} / \mathrm{FP}$ & $\begin{array}{l}0.316 \\
0.147\end{array}$ & $\begin{array}{l}191.5^{\ddagger} \\
187.7\end{array}$ & $\begin{array}{l}122.8^{\ddagger} \\
120.3\end{array}$ & $\begin{array}{l}191.5 \\
187.7\end{array}$ & 379.2 & 189.6 & 121.5 \\
\hline & (3@5.35)11-5-O4.5-0-i-2.5-3-19.25 & $\begin{array}{l}\text { A } \\
\text { B } \\
\text { C }\end{array}$ & CB & $\begin{array}{c}0.001 \\
- \\
-\end{array}$ & $\begin{array}{l}132.5 \\
127.5 \\
126.0\end{array}$ & $\begin{array}{l}84.9 \\
81.7 \\
80.8\end{array}$ & $\begin{array}{l}132.5 \\
127.5 \\
126.0\end{array}$ & 386.0 & 128.7 & 82.5 \\
\hline & (3@5.35)11-5-S5.5-0-i-2.5-3-19.25 & $\begin{array}{l}\text { A } \\
B \\
\text { C }\end{array}$ & $\mathrm{CB} / \mathrm{BS}$ & $\begin{array}{c}- \\
0.321 \\
0.105 \\
\end{array}$ & $\begin{array}{l}137.3 \\
140.5 \\
134.9 \\
\end{array}$ & $\begin{array}{l}88.0 \\
90.1 \\
86.5 \\
\end{array}$ & $\begin{array}{l}137.3 \\
140.4 \\
134.5 \\
\end{array}$ & 412.2 & 137.4 & 88.1 \\
\hline & (3@5.35)11-5-O4.5-6\#3-i-2.5-3-19.25 & $\begin{array}{l}\text { A } \\
B \\
C\end{array}$ & CB & $\begin{array}{c}- \\
- \\
0.042 \\
\end{array}$ & $\begin{array}{l}137.4 \\
137.1 \\
150.7 \\
\end{array}$ & $\begin{array}{l}88.1 \\
87.9 \\
96.6 \\
\end{array}$ & $\begin{array}{l}137.4 \\
137.1 \\
150.7 \\
\end{array}$ & 425.1 & 141.7 & 90.8 \\
\hline & (3@5.35)11-5-S5.5-6\#3-i-2.5-3-19.25 & $\begin{array}{l}\text { A } \\
\text { B } \\
\text { C }\end{array}$ & CB & $\begin{array}{c}- \\
- \\
0.020\end{array}$ & $\begin{array}{l}151.6 \\
157.4 \\
149.5\end{array}$ & $\begin{array}{c}97.2 \\
100.9 \\
95.8\end{array}$ & $\begin{array}{l}151.6 \\
157.4 \\
149.5\end{array}$ & 458.6 & 152.9 & 98.0 \\
\hline
\end{tabular}

${ }^{\ddagger}$ No anchorage failure on the bar 